\title{
Scalable Computing: Practice and Experience
}

\section{Scientific International Journal}

for Parallel and Distributed Computing

ISSN: 1895-1767



Volume 19(4)

December 2018 


\section{Editor-IN-CHIEF}

\section{Dana Petcu}

Computer Science Department

West University of Timisoara

and Institute e-Austria Timisoara

B-dul Vasile Parvan 4, 300223

Timisoara, Romania

Dana.Petcu@e-uvt.ro

MANAGINIG AND

TEXnical Editor

\section{Silviu Panica}

Computer Science Department

West University of Timisoara

and Institute e-Austria Timisoara

B-dul Vasile Parvan 4, 300223

Timisoara, Romania

Silviu.Panica@e-uvt.ro

Book Review EDITOR

\section{Shahram Rahimi}

Department of Computer Science

Southern Illinois University

Mailcode 4511, Carbondale

Illinois 62901-4511

rahimi@cs.siu.edu

\section{Software Review Editor}

\section{Hong Shen}

School of Computer Science

The University of Adelaide

Adelaide, SA 5005

Australia

hong@cs.adelaide.edu. au

Domenico Talia

DEIS

University of Calabria

Via P. Bucci 41c

87036 Rende, Italy

talia@deis.unical.it

\section{EDITORIAL BOARD}

Peter Arbenz, Swiss Federal Institute of Technology, Zürich, arbenz@inf .ethz.ch

Dorothy Bollman, University of Puerto Rico, bollman@cs . uprm.edu

Luigi Brugnano, Università di Firenze, brugnano@math.unifi.it

Giacomo Cabri, University of Modena and Reggio Emilia, giacomo.cabri@unimore.it

Bogdan Czejdo, Fayetteville State University, bczejdo@uncfsu.edu

Frederic Desprez, LIP ENS Lyon, frederic.desprez@inria.fr

Yakov Fet, Novosibirsk Computing Center, fet@ssd.sscc.ru

Giancarlo Fortino, University of Calabria, g.fortino@unical.it

Andrzej Goscinski, Deakin University, ang@deakin.edu.au

Frederic Loulergue, Northern Arizona University, Frederic.Loulergue@nau.edu

Thomas Ludwig, German Climate Computing Center and University of Hamburg, t.ludwig@computer.org

Svetozar Margenov, Institute for Parallel Processing and Bulgarian Academy of Science, margenov@parallel.bas.bg

Viorel Negru, West University of Timisoara, Viorel.Negru@e-uvt.ro

Moussa Ouedraogo, CRP Henri Tudor Luxembourg, moussa. ouedraogo@tudor. Iu

Marcin Paprzycki, Systems Research Institute of the Polish Academy of Sciences, marcin.paprzycki@ibspan.waw.pl

Roman Trobec, Jozef Stefan Institute, roman.trobec@ijs.si

Marian Vajtersic, University of Salzburg, marian@cosy.sbg.ac . at

Lonnie R. Welch, Ohio University, welch@ohio.edu

Janusz Zalewski, Florida Gulf Coast University, zalewski@fgcu.edu 


\section{Scalable Computing: Practice and Experience}

Volume 19, Number 4, December 2018

\section{TABLE OF CONTENTS}

Special Issue on Mobile Cloud Applications and Challenges:

Introduction to the Special Issue

A Survey on Mobile Cloud Computing: Mobile Computing + Cloud

Computing $(\mathrm{MCC}=\mathrm{MC}+\mathrm{CC})$

Ramasubbareddy Somula, Sasikala R.

Execution Analysis of Spatial Data Storage Indexing on Cloud

Environment

Karthi S., Prabu S.

Enhanced Data Security for Public Cloud Environment with Secured

Hybrid Encryption Authentication Mechanisms

Prabu S., Gopinath Ganapathy, Ranjan Goyal

Cloud based Dynamic Course Selection Framework using Network

Graphs with Term Difficulty Estimation

Jasem M. Alostad

Parallel Seed Selection Method for Overlapping Community Detection

in Social Network

Belfin R.V., E. Grace Mary Kanaga

\section{Regular PAPERS:}

Energy-Efficient Real-Time Scheduling Algorithm for Fault-Tolerant

Autonomous Systems

Hussein EL Ghor, Julia Hage, Nizar Hamadeh, Rafic Hage Chehade

Modelling and Simulation of GPU Processing in the MERPSYS

Environment

Tomasz Gajger, Pawet Czarnul

A Comparison of Message Passing Interface (MPI) and Co-array

FORTRAN for Large Finite Element Variably Saturated Flow

Simulations

Fred T. Tracy, Thomas C. Oppe, Maureen K. Corcoran 



\section{INTRODUCTION TO THE SPECIAL ISSUE ON MOBILE CLOUD APPLICATIONS AND CHALLENGES}

We are happy to present this special issue of thee Scientific Journal Scalable Computing: Practice and Experience. In this special issue on Mobile Cloud Applications and Challenges (Volume 19 No 4 December 2018), we have selected five papers, which gone through a peer review according to the journal policy. All the papers represents novel results in the field of Mobile and Cloud Applications.

The first paper presents overview of mobile cloud computing, cloudlet technology, security and privacy issues and limitations of mobile cloud commuting. The second paper presents a spatial partition, global index and map reduce operation were studied. The trail results that the proposed indexing cloud framework performs improved results. The third paper proposes an authentication model along with data security in a public cloud storage environment which successful detects the unauthenticated access or any anomaly in data. The fourth paper analyses the student success ratio which uses a cloud based technology to implement and design SaaS. Graph based complex network are used for analysing the course. The fifth paper presents Overlapping community detection in social networks. The proposed algorithm uses parallel processing engine to resolve the delay problem.

We use this opportunity to thank all the contributors to this special issue. We would like to express our special gratitude for the Editor-in-chief, Professor Dana Petcu for her constant support for carrying this special issue.

Rajkumar Rajasekaran, Vellore Institute of Technology, India 



\title{
A SURVEY ON MOBILE CLOUD COMPUTING: MOBILE COMPUTING + CLOUD COMPUTING $(\mathrm{MCC}=\mathrm{MC}+\mathrm{CC})$
}

\author{
RAMASUBBAREDDY SOMULA*AND SASIKALA R $\mathrm{R}^{\dagger}$
}

\begin{abstract}
In recent years, the mobile devices become popular for communication and running advanced real time applications such as face reorganization and online games. Although, mobile devices advanced for providing significant benefits for mobile users. But still, these devices suffers with limited recourses such as computation power, battery and storage space due to the portable size. However, The Cloud Technology overcome the limitations of mobile computing with better performance and recourses. The cloud technology provides enough computing recourses to run mobile applications as storage computing power on cloud platform. Therefore, the novel technology called mobile cloud computing (MCC) is introduced by integrating two technologies (Mobile Computing, Cloud Computing) in order to overcome the limitations(such as Battery life, Storage capacity, Processing capacity) of Mobile Devices by offloading application to recourse rich Remote server. This paper presents an overview of MCC, the advantages of MCC, the related concepts and the technology beyond various offloading frameworks, the architecture of the MCC, Cloudlet technology, security and privacy issues and limitations of mobile cloud computing. Finally, we conclude with feature research directions in MCC.
\end{abstract}

Key words: Mobile Computing, Cloud Computing, Mobile Cloud Computing, Cloudlet Selection, Computation offloading, Edge Computing, security.

AMS subject classifications. 15A15, 15A09, 15A23

1. Introduction. Over the past few decades, mobile devices have been playing an important role in our modern and virtual lifestyle. For instance, according to the survey by International Data Corporation (IDC) in 2016, the usage of mobile devices and tablets was increased by 1.6 billion units exponentially [1]. In recent years, mobile applications became popular in various categories such as news, entertainment, health, business and social networks. The mobile computing allows users to access all necessary applications from application centres such as Android play store and Apple iTunes etc., irrespective of location. Even mobile cloud computing provides high-end features for running various real time applications but still users demand for more computing resources. For mobile computing, the mobile devices are designed with limited battery life, storage capacity, processing capacity and communication capabilities. Mobility is important feature on pervasive computing environment where the user is able to perform his work without any interruption. The cloud computing is emerging technology which is formed with amalgamation of various technologies such as virtualization, distributed computing, SOA, web services etc. Cloud computing provides massive computing resources (such as hardware, software, storage) in order to improve the performance of application as well as reducing processing cost. It allows users to access data from any location on demand basis. The mobile device will perform high computational tasks on cloud platform which require more computing resources. The cloud computing paradigm can be represented through three different service models. Platform as a service (paas), Infrastructure as a service (Iaas), software as a service (saas) as shown in Fig 1.1The author in [2], presented the annual growth rate of cloud service models, Iaas is $41 \%$, paas holds $26.6 \%$ and Saas holds $17.4 \%$. The emerging technology, Mobile Cloud Computing has been introduced to overcome limitations of mobile devices. Recently, the mobile users demand for computing is being increased due to the development in mobile computing technology. Various studies define the importance and benefit of mobile cloud computing for mobile users and enterprisers. For example, according to the ABI, the usage of mobile devices reached to 280 million by 2015 , the revenue of Mobile cloud computing reached to $\$ 5.2$ billion [3]. Currently the growth of advanced mobile devices developed rapidly with sufficient resources such as battery life, storage, processing power. Nonetheless, it is still suffering from processing real time application such as image recognition,video streaming, language translation. Mobile devices are less compared to server systems and desktop computers in terms of computing power and storage. When mobile device runs resource intensive task put heavy load on processor and reduce battery life.

* School of Computer Science and Engineering (SCOPE), VIT University, Vellore, India (svramasubbareddy1219@gmail.com).

${ }^{\dagger}$ School of Computer Science and Engineering (SCOPE), VIT University, Vellore,India.(sasikala.ra@vit.ac.in) 




FIG. 1.1. Cloud Computing Services

Nowadays, the research work on cloud computing is aiming to enhance computing capabilities of mobile devices by allowing Mobile users to access various service based models such as software, infrastructure and computing services. Amazon is one of the cloud service provider which provides security to user personal data by various storage service models (S3) [4]. MCC promises to improve performance of the mobile application beyond mobile computing, with the help of cloud computing [5] [6]. Most of the data generated by the mobile device will be video content which is over $78 \%$ by 2021 forecast by cisco [7]. The concept of offloading fully or part of the application into remote cloud environment to address limitations of mobile computing through service providers other than the mobile can deploy application on cloud where both storage and computation can happen out of mobile device is known as Mobile Cloud.

In this paper, we aim to discuss Various categories of research areas in Mobile Cloud Computing such as computation offloading, cloudlet selection (or) edge computing, resource provisioning, security, privacy issues and VM migration techniques. Furthermore, we plan to discuss proposed research works and also upcoming novel solution for addressing Mobile Cloud Computing issues.

\section{Mobile Cloud Computing Overview.}

2.1. Definition of Mobile Cloud Computing. MCC is an emerging technology where it fill gap between limited resources of Mobile devices as well as resource intensive applications required to run a resource rich environment computing. According to the MCC forum definition: the execution of mobile application will happen outside of mobile device. The computation power and storage of mobile application more to cloud environment for processing. The MCC allows mobile users to access computing services, it is not restricted to particular mobile users [3]. In the second definition [8] [9]. The mobile cloud computing provides computing resources for mobile devices remotely. In the third destination [10] [11], the cloud server does not need to act as powerful server. But enhancing mobile devices configuration setup in terms of storage and processing capacity.

\subsection{Related concepts and technology.}

2.2.1. Mobile Computing. Nowadays, Mobile Devices (MD) became an essential equipment for communication in everyone life. Even though mobile devices are able to support real time resource hungry applications but still they are limited in terms of storage, processor and power consumption. In order to address this issues, 
the emerging cloud computing technology provides enough resources to optimize performance of the mobile applications. Generally, mobile computing is a process of executing applications in mobile device and transferring result to one (or) more devices. Mobile communication is able to make use of centrally located application (or) data with help of small (or) little portable computing devices. This technology make every application to be executed in single devices. The usage of mobile devices is increasing day by day, the requirement is to provide better services for low cost and power consumption also increases. The list of issues in mobile computing is represented in following Fig 2.1.

2.2.2. Mobile Network Architecture. The classification of mobile network can be represented as following Fig 2.2.

2.2.3. Cellular Architecture. Earlier, the mobile networks were intended to cover huge geographical area by using single transmitter with high power consumption. Even conventional architecture covers huge geographical area, but it does not support frequency reuse technology.

In order to facilitate frequency reuse as well as large coverage, the cellular architecture was brought into mobile networks. This cellular architecture replaces high power consumption transmitter with low power transmitters. The large geographical area splits into number of hexagonal cells which are served by base station.

Each cell in cellular network is surrounded by number of independent cell. Each adjacent cell boundary touches each other. The hexagonal cell covers certain area in geographical location. Each cell is served by nearby base station. The base station which serves each cell is allotted with certain portion of frequency. The base station of adjacent cell is allotted with different frequency ranges to overcome interruptions in communication.

The following formula depicts the frequency reuse distance:

$$
d=r \sqrt{(3 * n)}
$$

Here, $r$ represents distance between cell center and cell boundary

$n$ represents adjacent cells around concerned cell

2.2.4. Mobile Ad Hoc Network Architecture (MANET). In MANET's network, nodes, routers and switches position are not fixed. It consists of mobile devices which communicate with each other through wireless network. In such network, the node is able to service and send or receive response from nearby neighboring node. The positions of nodes in MANET can organize the network.

The following Fig 2.3 illustrates the behavior of the MANET architecture. It consists of 5 nodes, two are mobile nodes (mobile node $1 \&$ mobile node 2 ). One is to handle pc and the other is sensor node. The base station acts as a router which routes messages to all involved nodes to MANET network.

Each node in MANET behaves like router to communicate with other neighboring nodes. It is also known as self-organized network [12].

2.2.5. Mobile Wireless Sensor Network Architecture (MWSN). MWSN is similar to the MANET network, except sensor nodes involvement. In MWSN, the sensor nodes having computing and communication abilities [12].

In MWSN, the sensor nodes acts as routes to pass messages to neighbour nodes as well as to communicate with other networks such as MANET, cellular network Fig 2.4 depicts the MWSN architecture.

The main advantage of MWSN over static sensor network is the expansion of no. of applications. It is used in many real time applications such as health care to monitor blood pressure and heart rate [12].

2.3. Cloud Computing. Cloud Computing (CC) is an advanced technology that provides computing resources for Information Technology (IT) to increase capability and capacity over network [6]. Cloud is a collection of virtualized computers which provides resources dynamically on basis of pay as you go (or) pay-per use model [12]. Cloud computing allows users to access application (or) data anytime from anywhere on demand basis [6]. It is mainly focusing on development of advanced applications, computing models and using existing services for developing new software [13]. Cloud computing technology is composed of various technologies such as grid computing, SOA, virtualization, web services.

Various applications with client as a model. We can say Amazon web services (AWS) and Microsoft Azure cloud as example of public cloud. Azure cloud open and provide services to build, deploy and run applications 


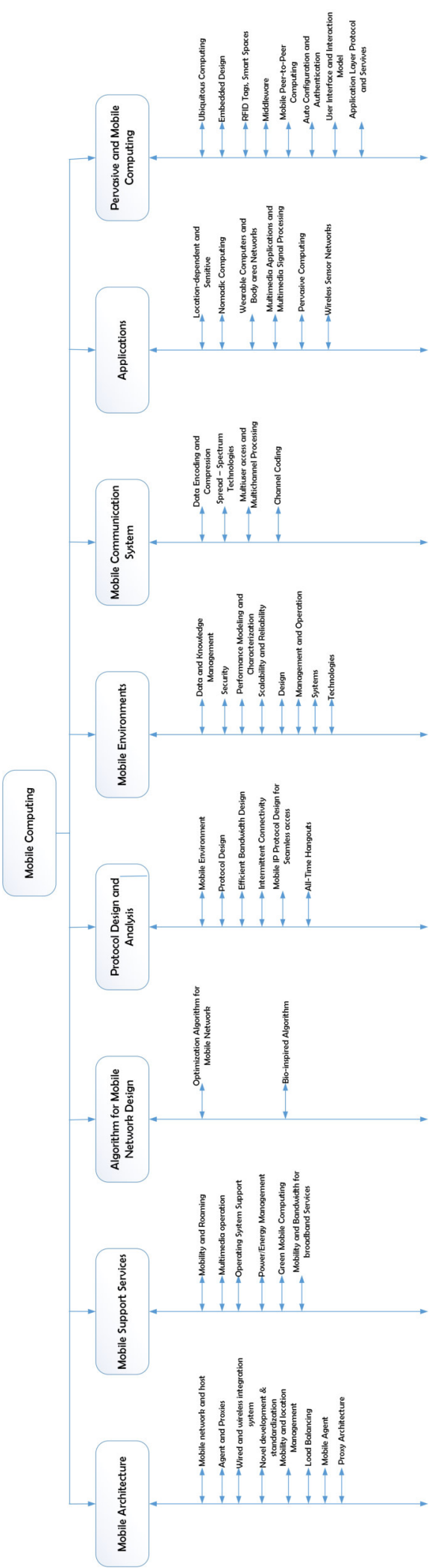

FIG. 2.1. Mobile Computing Challenges 




Fig. 2.2. Classification of Mobile Network



FIG. 2.3. Architecture of MANET

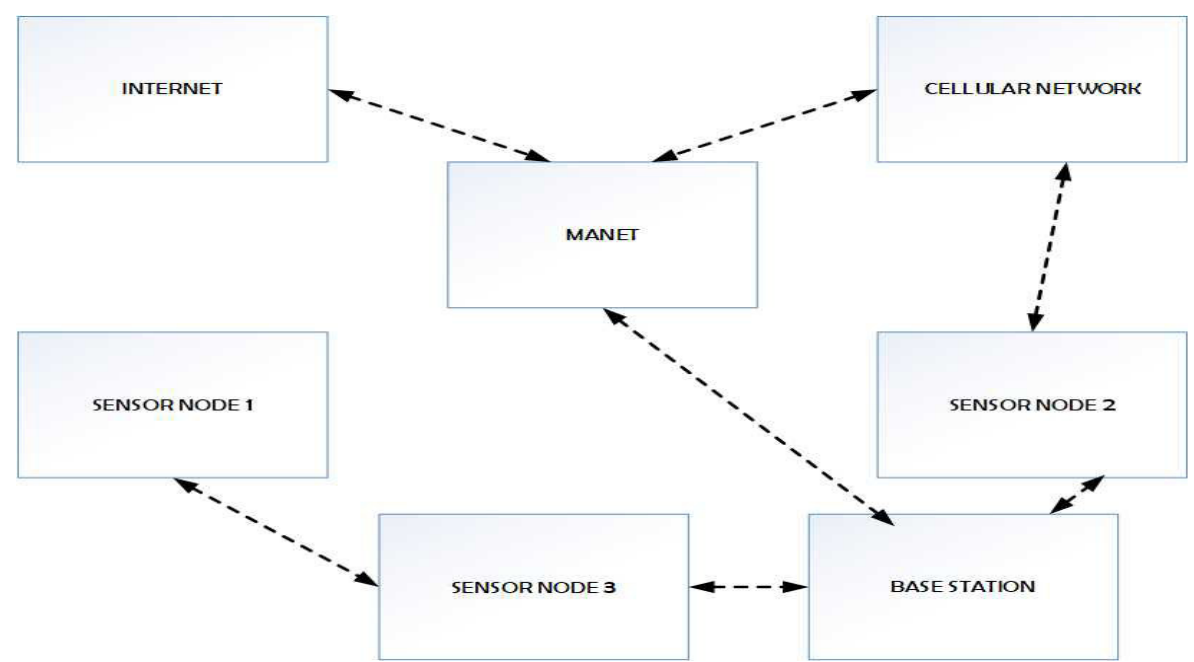

FIG. 2.4. Wireless Sensor Network Architecture 
on servicers [4]. AWS cloud provides services via two models, Infrastructure as a Service (Iaas) and software as a service (saas). The client can directly access applications without installing any software's in local system [14].

\subsubsection{Characteristics of cloud computing.}

On demand self-service. Whenever user client require services such as virtual machine for processing and storage is being leveraged without any interaction between users and service providers.

Broad Network Access. Client can access services at anytime from anywhere through powerful devices such as laptops, smart phones and tablets.

Resource Polling. Multiple users can access computing resources (processing power, storage, bandwidth, memory) in multi-tenancy model. The user have no information about from where the services are provided by service provider.

Rapid Elasticity. Based on subscriber demand, the resources are rapidly increased Automatically. The user thinks that cloud resources are limited and scalable at any time.

Measured Services. The service provider offer resources on pay-per-use Manner. the transparency is to be maintained between users and service providers.

2.4. Cloud Computing Deployment Models. Cloud models can be used to deploy cloud services. The deployment models are classified into four types [15] [14] [16].

1. Private cloud;

2. Public cloud;

3. Hybrid cloud;

4. Community cloud.

Private Cloud. In this model, data center is owned by particular organization and managed by either organization or third party. Private cloud is restricted to particular users [17].

Public cloud. Public cloud is not restricted to particular user. It can be used by all kinds of cloud users such as Research, Industry and Company [17].

Hybrid cloud. In this model, one or more deployment models are integrated to design single data centre. This model can be used to overcome issues arises by private cloud during accessing [14] [18] [19] [16] [17].

Community cloud. In this model, the data centre is owned by one or more organizations and managed either by third party or only one of the community organization.

2.5. Architecture of Mobile cloud computing. Nowadays mobile devices became part of our daily life style and can be connected to any cloud server at anytime from anywhere via wireless infrastructure. Cloud computing introduced concept: Bring Your Own Device(BYOD), which allows employees to leverage privileged organization content and applications deployed in cloud server. The virtualization technology in cloud computing enables multiple VMs (Virtual Machines) or operating systems to run on smart phone devices including tablets, smart devices and laptops. That is cloud computing provides services in multi-tenant manner to subscribers via mobile virtualization. The cloud computing offers task oriented services with virtualization on mobile devices to provide unlimited computing power and storage on demand basis. Cloud computing build MCC applications which are enhanced in terms of computing power and storage comparing with traditional mobile computing applications.

Limited Battery Life. The battery capacity of mobile device is limited to run high-end application. It is not possible to depend on other external power sources while moving (mobility). The charge of battery will be lost in few hours.

Limited storage capacity. Every smart phone or mobile device is configured with 8 GB and laptop is configured with 500 GB. It can be expanded with external memory. It cannot support more than configured storage, when back is required.

Limited processing capacity. The smart phone having ARM processor, it can only run small and very few applications. In case of laptops with various processor (i3, i5 and i7) are available but not affordable due to high cost. The processor in mobile device cannot be upgraded if anyone want to upgrade. 




FIG. 2.5. Architecture of $M C C$

Low Bandwidth. The conventional technologies such as EDGE, GPRS and GSM provide low bandwidth. The advanced technologies $3 \mathrm{G}$ and $4 \mathrm{G}$ provides high bandwidth, but they are available only in developed cities/towns. Fig 2.5 depicts the architecture of MCC which is categorized into three different layers:

1. User layer;

2. Network layer;

3. Service provider layer.

\subsection{Benefits of Mobile Cloud Computing.}

2.6.1. Extended battery lifetime. Battery consumption became a serious issue in mobile computing. There have been many proposed models in order to address battery issue. But they are focused on hardware design. MCC provided solution for preserving battery life by offloading resource intensive applications onto cloud and then the entire process will be done at cloud side after that result is sent back to mobile device.

2.6.2. Data Storage. According to user perspective, MCC provides unlimited storage on demand basis. Cloud storage permits users to store and access data from anywhere at any time. The data stored in cloud could be any multimedia data. The cloud storage stores data in an encryption format if any change causes to mobile, the data will remain safe in cloud as a backup.

2.6.3. Increasing processing power. The computing power could be saved in mobile device by processing applications on cloud and result depicted in device. The mobile user does not feel of having limited processing power because cloud provides unlimited resources. the MCC allows users run complex resource intensive applications without any resource restrictions. 
2.6.4. Dynamic provisioning. Mobile users can access required resources on demand basis, dynamic provisioning that permits users to have access resources without any advanced reservation by creating virtual machines (VMs) with appropriate configuration. Whenever user occurs cloud services, the no. of CPU cores and storage dynamically increased based on requirement. The self service provisioning is more beneficial compared to hardware configuration enhancement.

2.6.5. Scalability. Scalability is one of the significant characteristics of cloud computing. The resources allocated to user will be increased or decreased based on user requirement. The cloud service provider ensure to manage resource requirement of mobile application.

2.6.6. Reliability. Cloud is always reliable compared to mobile device. Cloud renders provide security application such as virus scanning and malicious code detection being executed in cloud. In order to save user from installing in local systems, MCC provides various authentication mechanisms for preventing unauthorized user from access cloud resources or confidential data.

2.6.7. Ease of integration. In mobile computing environment, the user cannot access resources or services. In MCC, the user can access all kind of services due to integration of various services into cloud. The emerging advanced technologies such as Big Data and IOT can be easily integrated with MCC technology to enhance the Quality of Services (QOS).

3. Offloading Approach. The concept of offloading can be done by offloading resource intensive application partly or fully from mobile device (MD) to cloud. Offloading classified into two ways namely code offloading and state offloading. Code offloading is achieved through sending part of the application to remote cloud for executing. On other hand, state offloading means transferring entire application to remote cloud. The process of offloading can be achieved by following three steps:

1. Partitioning

2. Preparation

3. Offloading decision

Partitioning. Partition of an application is an initial step in which the entire application is divided into various components. These components are affordable and non-affordable which means the components run on local device or run at remote cloud server. Based on different information the component can be considered either affordable or non-affordable. While designing application the programmer annotate local or remote execution through an API as affordable. The intensive part of application can be identified through code analysis and performance prediction (application profiling). It is not efficient approach partitioning application at designing time, because both techniques are not considering real-time execution context. So that the accuracy is very less.

Preparation. In this step, the actions which are required for execution of mobile application at remote server. This action may be selection of server, installation of code and execution on account of mobile device. Both data and code needed for remote execution.

Offloading decision. This is final step in offloading, before offloading component onto remote server. When mobile device uses offloading component then it is not necessarily to depend on execution. The decision is based on run time, then the real time information available such as battery consumption for sending data to remote server, wireless connection strength. Comparatively the runtime includes more overhead than decision during design time.

3.1. Types of Frameworks. The offloading frameworks can be classified into two categories. The first category is static offloading frameworks. In which all discussed steps in above section can be achieved at design time on other hand, the dynamic offloading framework can be achieved at runtime. It means the decision is taken at runtime whether to offload or not.

3.2. Offloading Mechanism. There have been various proposed works on offloading mechanism for offloading resource intensive application into cloud. This can be classified into two offloading mechanisms:

1. VMs offloading

2. Code offloading 
In code offloading, the computational intensive component is sent to remote server by invoking Remote Procedure Call (RPC) with the help of notations, compilers and binary code modifiers where as VMs offloading can be achieved capturing mobile state and storing into cloud. During offloading the execution is stopped at mobile device and VM clone sends to cloud.

3.3. Comparison among various offloading mechanisms in MCC. The existing popular offloading mechanisms would be discussed in this section. Each mechanism properties and offloading process concludes at end of the section [20].

3.3.1. Clonecloud Framework. The motivation behind clone cloud [21] is to reducing power consumption on mobile device by offloading computation intensive application into remote server. In clone cloud, the application partitioning can be achieved by integrating program profile with program partitioning in order to obtain constrains, for example the component which depends on local mobile device integrates parts like sensor , camera and speakers can be executed locally. The clone cloud mechanism will use threads functionalities at application portioning. The programming analysis aim to obtain possible migration points, in other hand the profile is aimed to produce cost of migrating and processing at server.

In preparation step, the application of the mobile device is captured and stored in cloud server.

In decision step, the decision is taken place at runtime which means all running threads on mobile device are suspended and transferred to cloud server then all threads resumed in clone cloud to offload computation. The execution process in clone cloud is to create duplicate mobile software in cloud server. Then computation is offloaded to server and result $\mathrm{s}$ back later once the execution process is done. The distributed mechanism in clone cloud aims to implement partitioning process for given application in application layer virtual machine $(\mathrm{VM})$.

The author Chan et al [21], tested clone cloud with different applications such as virus scanner, image search and privacy preserving applications in various scenarios for example, clone cloud with Wi-Fi and clone cloud with $3 \mathrm{G}$ environment.

3.3.2. MAUI Framework. The MAUI [21]framework focusing on energy optimization by executing complex components at server in cloud. The execution of components in MAUI is done dynamically because continuous profiling process. The MAUI tries to hide the difficulties of execution at remote server from mobile user in order to make an impression that the entire application execution is done at local device. The developer of application can decide the annotations which component is to execute in mobile device or which is to execute at remote server.

In order to achieve MAUI partitioning framework the following conditions must be installed in both mobile device and remote server side. One is application binaries and other one is proxies, profilers and solvers.

The profile maintain information about network conditions which is helpful for MAUI to take appropriate decision otherwise leads to wrong decision. The profiler keep on updating the information during whole execution of the application.

In MAUI, the profiler will collect information and gives it to the MAUI solver which can make decision at runtime whether to offload or run it locally. Author has conducted various experiments using three various applications such as face recognition, chess and video. In first comparison, the author has composed energy consumption of application on stand-alone mobile device and with MAUI framework. The energy consumption is optimized with MAUI framework by offload nearby server. On other hand, the energy consumption is reduced by executing chess and video game respectively $45 \%$ and $25 \%$ with MAUI framework by offloading to nearby server.

3.3.3. Cloudlet Framework. Offloading mechanism is not always optimum solution because of network failure and long processing delay. The cloudlet is a cloud in box, which is situated nearby mobile device. We can say that cloudlet brings cloud closer to mobile devices.

The cloudlet reduces response time in milliseconds by executing application in nearby cloudlet that is comparatively better than executing on remote cloud server. Satyanarayan et al in [22], introduced VM based cloudlet framework in which, cloudlet for hosting offloaded task that is run on remote server for storage and processing purpose the cloudlet is not as same as cloud and any other parallel system. The cloudlet based VM supports scalability, mobility and elasticity. 
In preparation step, the cloudlet framework require mobile device application processing environment at remote server then offload complete application to remote server through VM which is based on dynamic VM synthesis. The mobile device act as interface and the entire application execution can be achieved at cloudlet infrastructure. The user mobility is a primary challenge while processing application in cloudlet.

The cloudlets are distributed in geographical area, the users can easily access storage resources and computing cycles via internet infrastructure. In order to avoid long delays, generally cloudlets located at population areas such as bus stops, coffee shops and colleges. Users can access distant cloud via cloudlet if the user must offload resource intensive application, then the application has to discover and send application to cloudlet [22], otherwise the application can select optimal cloudlet based on network status.

3.3.4. Jade Framework. Jade framework [23] is similar to other framework, but different perspective. In jade, the system consider both application and device status to make appropriate decision where the application must be executed. This framework aims to reduce energy consumption for mobile devices as well as minimize burden on application developer.

The application is partitioned into various classes based on available information. In partition step, the system verifies both application and device status through other information such as load variation, energy level communication cost. The jade designed with enough number of APIS, this minimize the burden on developer to control the partition of application and remote server interact with local code.

In jade, the offloading decision is taken at run time whether to execute locally or remote server. Jade can be performed on two different servers.

1. Android server

2. Non-android server

The non-android server must maintain installation of java platform. Jade runs as normal java program at nonandroid server. The decision of offloading can be changed based on device status in result, energy consumption is reduced. Jade can easily transform computational task from mobile device to available remote server to optimize energy consumption.

The author conducted experiment using face recognition application. The experiment was done on 50 pictures each with $200 \mathrm{~kb}$ size. In result, jade has outperformed on existing frameworks by reducing $34 \%$ average power consumption.

3.3.5. Mirror Server Framework. This mirror framework [24] use Telecommunication Service Provider (TSP) at remote server. TSP would provide services to landline mobile users. The mirror server can advance the mobile devices by providing required resources storage, computation offloading and security on computation infrastructure. The mirror server can maintain VM instances for various mobile devices. In mirror framework, the entire application is offloaded to remote server so the partition of application not necessary.

In preparation step, the new virtual machines (VMs) created, this VMs are managed and deployed by Mirror server. The application execution is done at Mirror VM instance under control of mirror server. Mirror server optimize offload mechanism.

The mirror server is not specially designed for data analysis and provide limited services (i.e. file sharing and file scanning) are included. The author conducted experiments by installation of file scanner at mirror server. The applications are trying to access mirror. The energy is reduced considerably, execution time also increased running scanner on mirrors.

3.3.6. Cuckoo Framework. The author in [25], has introduced new framework called as cuckoo, which offload resource-intensive code to remote server for mobile device. In this model, offloading can be achieved through java stub model. Cuckoo was designed to advances the performance and reduce battery utilization. The partition of application is adapted from existing android model, which separates affordable and non-affordable components of the application. This process represents through user interface. The affordable components are offloaded into any JVM resource. In preparation step, the application developer is required to write code two times, one for local execution and other is for remote execution. For this the require programming model which is useful when connection is dropped support execution. This both codes combined to form single package. Cuckoo framework is dynamic offloading model and offload only well-identified components of application. If remote resource is not available for offloading task then execution will takes place in local device. 
3.3.7. Phone2cloud Framework. In phone2cloud [26], the author has focused on energy efficiency and application performance by conducting quantitative experiments on various scenarios. This framework is not fully automatic offloading framework, if application needs to be executed over cloud then it requires to modify manually at preparation stage. The delay-tolerance threshold and static analysis are required to make offloading decision. This threshold can be formed based on prediction of time taken for transferring data to remote cloud via Wi-Fi network. This framework waits until Wi-Fi available rather than sending data directly.

The phone2cloud framework aimed to reduce power consumption and execution time while offloading. It was implemented on Android and Hadoop environment to analysis experiment results. There are various components involved in phone 2cloud framework, which would be helpful to make appropriate offloading decision to run either locally or remotely. Components like bandwidth monitor, resource monitor, offload decision manager, remote execution manager, offloading predictor, local execution manager and offloading proxy.

The framework consider two parameters before offloading takes place. First one is execution time of application on mobile device and delay tolerance threshold. Second one is power consumption of application to run on both mobile device as well as cloud environment. If the user waiting time is (delay tolerance threshold) is less than average execution time on mobile device then application offload to cloud. The power consumption is also considered as another constrain before application is offloaded to cloud. If power consumption is less for executing in mobile device, then application run locally.

The author examined phone2cloud by conducting experiments with various applications such as word count, path finder and sort application. In result, framework reduced energy consumption, improves preference of application and experience of the user.

3.3.8. Thinkair Framework. ThinkAir framework aims to address issues raised by existing frameworks such as MAUI does not address issue of scalability while executing application over cloud. The clonecloud framework tries to extend binary pieces of process to make overall execution faster on cloud.

However, this approach will not support if any drastically changes happen to input or execution environment. In order to address these two challenges such as elasticity or scalability and power consumption in mobile device, the author in [27] introduced thinkair framework.

In preparation step, the methods are annotated as remote which are received to offload cloud server. Thinkair approach provide simple programmer API to reduce burden on application developers. The execution component can detect whether method is affordable or not and handle all other necessary tasks such as decision making and communication with remote server without developer involvement.

The execution encounter makes decision for the first method based on environment parameters such as Wi-Fi signal, available sources. For example if the Wi-Fi signal is good then the method offloads to remote cloud otherwise executes on local mobile device. In profiling step, thinkair aims to predict make more accurate decision with the help of variant profilers such as hardware profiler, software profiler and network profiler. These three profilers together feed to estimate power consumption more accurately.

\section{Hardware profiler.}

- The hardware profiler collects information related to hardware such as CPU, screen, 3G and Wi-Fi interface.

- The CPU utilization is measured from 1 to 100 in different frequencies.

- Screen can be measured through brightness from 0 to 255.

- The power consumption of Wi-Fi interface either low or high.

- The $3 \mathrm{G}$ interface is either idle on shared with other channel.

Software profiler. The software profile collects information related to execution of program. This profile record following information:

- The total time required for executing method.

- The total CPU time of the method.

- No. of statements exist in method.

- No. of times the method invokes.

- The size of the thread.

Network profiler. Network profile involves overhead because it considers other profiles and parameters as well. In previous model, we used to consider only RTT on network. Now, thinkair brings other parameters such 
as number of packets sent/received per second, other parameters related to 3G/Wi-Fi interface, for example uplink and downlink rate for transferring and receiving data. These all measurements feeds to achieve better estimation in offloading method.

In thinkair, the partition is done manually by providing programmer API. The offloading decision can be made by considering variant profiles data. The author evaluated thinkair framework using four different applications such as face detection, N-queens Problem, virus scanning application and image merging application. The thinkair outperform in each experiment and reduced energy consumption and improved application performance using accurate prediction model.

3.4. Comparison Table among Different Offloading Frameworks. Table 5.1 presents our comparison between different offloading frameworks.

4. Cloudlet: Bringing cloud closer [28]. Nowadays mobile devices gaining popularity for computation and storage capabilities. The applications on mobile devices require more resources to process, but mobile devices due to lack of resources unable to provide required resources for resource-intensive applications. In Mobile Cloud Computing (MCC), computing offloading mechanism address resource hungry application by executing partly or entire application on remote cloud server. The offloading approach also faces challenge such as low bandwidth and high latencies. The computation offloading approach is not appropriate for realtime application such as face recognition, navigation and online video games. When network connectivity is poor then performance of application is affected. In order to address this problem, the cloudlet concept has been proposed by satyanarayan [22] [29] [30]. Cloudlet aims to bring cloud closer to mobile users [31]. Cloudlet is a sort of mini cloud which is formed by connecting various nearby mobile devices via Wi-Fi or Bluetooth. The mobile devices which are involved in cloudlet termed as cloudlet nodes. The cloudlet nodes could be laptops, mobile devices, PDAs, tablets and palmtops. Cloudlet allows nearby mobile users to leverage available computational resources via Wi-Fi network. Therefore, the execution time of application is reduced to milliseconds comparatively less execution on remote cloud server. The cloudlet is dynamic in nature, it can move and join at any point of time from network [32].

Nearby mobile users leverage cloudlet resources by running all resource-rich application and reduces endto-end response time [33]. Cloudlet can act as static cloudlet and dynamic cloudlet. The static cloud is termed as cooperative cloudlet because established by cooperative organization. Besides, cloudlet can be formed with nearby mobile devices such as device connected each other in railway station. Cloudlet is a novel emerging technology for latency-sensitive application and computation intensive application to improve application performance and user experience with application [34] [35]. Fig 4.1 represents basic process of cloudlet concept.

4.1. Cloudlet characteristic. The purpose of mobile cloud is discussed in above section that brings cloud resources close to mobile users. The functioning of cloudlet can be represented through following four characteristics briefly.

Soft-state. Generally, soft state is represented for efficiency in computer science, which can be replaced at any point of time. Soft-state is self-managing. It is completely different from hard-stated and holds catch state for cloud. Soft state store all mobile users data in buffer for security concerns before transmitting to remote cloud. Soft-state implementation is much more efficient in network environment when compared with hard-state.

Close at hand. Cloudlet is available very close to mobile users in order to provide high bandwidth and low latency in network.

Well Connected. Mobile Cloud Computing enhance battery power utilization by providing sufficient computational resources to processor offloaded resource rich mobile application over cloud.

Cloud Standards. Cloudlet functions as similar as remote cloud. The only difference is in bringing cloud resources to mobile user for reducing battery consumption and high latency issues. The offloaded task is executed on VMs running in cloud infrastructure.

4.2. Classification of cloudlet. Cloudlet can be classified into two types:

1. Ad-hoc cloudlet;

2. Elastic cloudlet. 


\begin{tabular}{|c|c|c|c|c|c|c|c|c|}
\hline . & 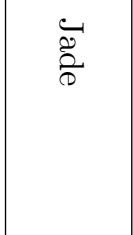 & 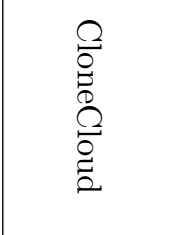 & $\begin{array}{l}\text { है } \\
\text { हैं } \\
8\end{array}$ & $\begin{array}{l}3 \\
\vdots \\
0 \\
0 \\
0 \\
0 \\
0 \\
9 \\
9\end{array}$ & $\stackrel{3}{\stackrel{3}{S}}$ & $\begin{array}{l}\sqrt{0} \\
0 \\
0 \\
0 \\
0 \\
0 \\
0 \\
0 \\
0\end{array}$ & 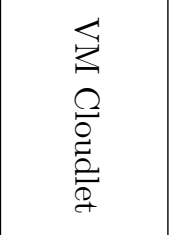 & 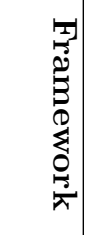 \\
\hline 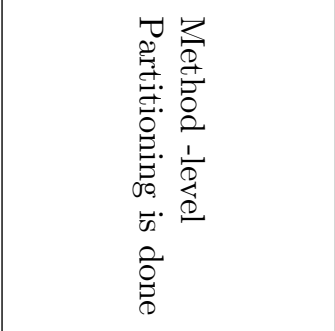 & 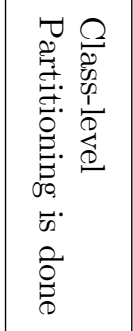 & 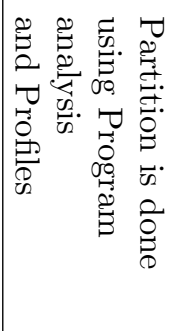 & 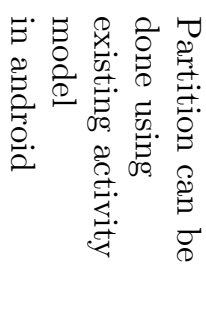 & 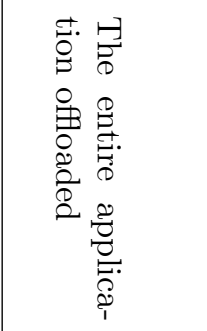 & 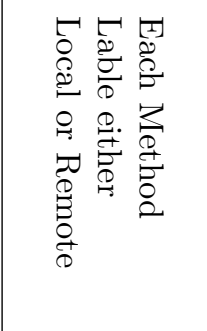 & 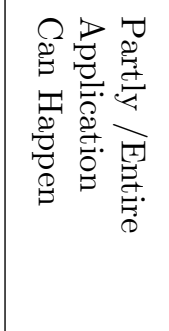 & 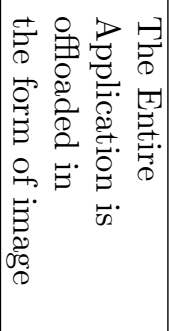 & . \\
\hline  &  &  &  &  & 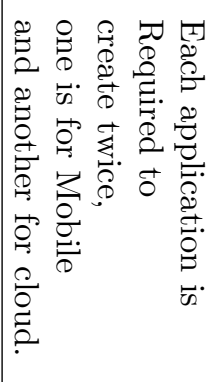 &  &  & 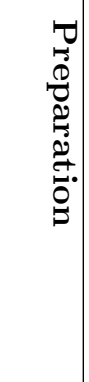 \\
\hline  &  &  &  & 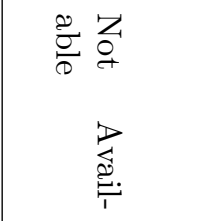 & 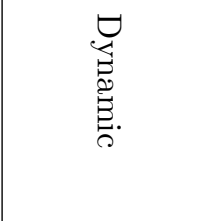 &  & $\begin{array}{l}z \\
0 \\
0 \\
\forall \\
8 \\
\stackrel{0}{0} . \\
0 \\
0\end{array}$ &  \\
\hline 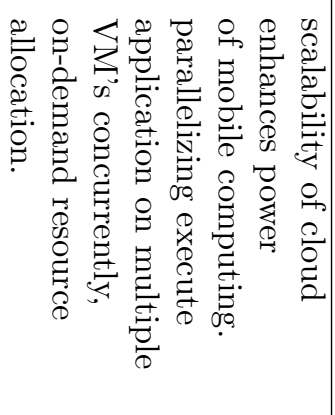 & 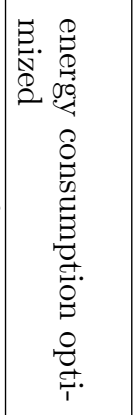 & 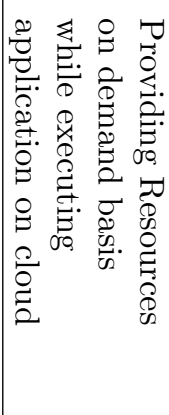 & 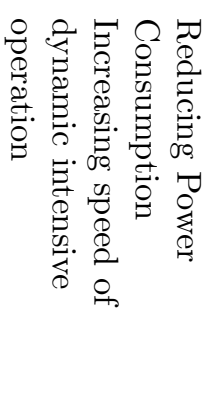 & 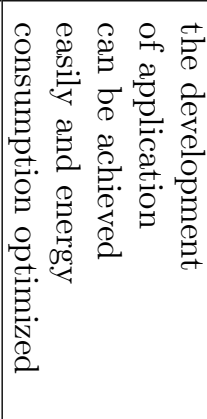 & 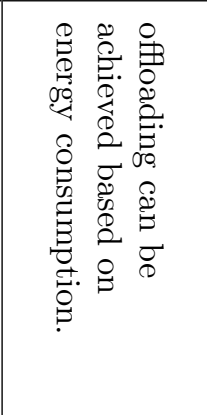 & 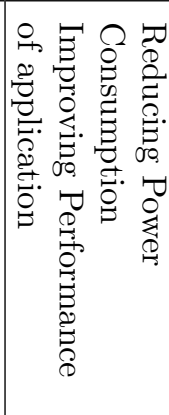 & 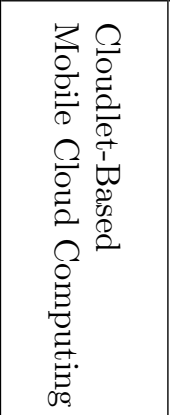 & 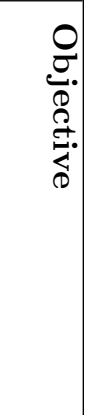 \\
\hline 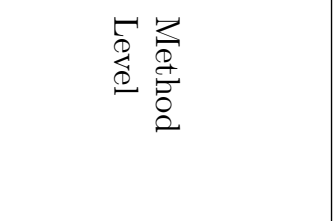 & 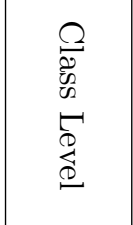 & $\begin{array}{l}\overrightarrow{7} \\
\stackrel{0}{0} \\
\stackrel{2}{2}\end{array}$ & 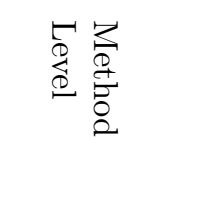 & 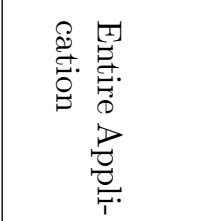 & 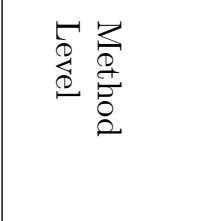 & 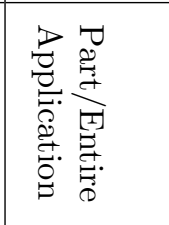 & 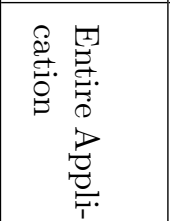 & 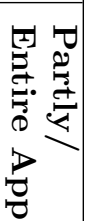 \\
\hline  & 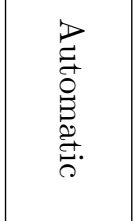 & 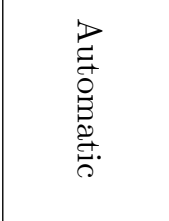 & 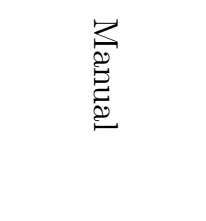 & 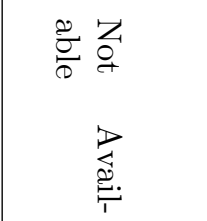 & 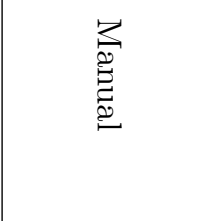 & 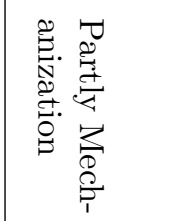 & 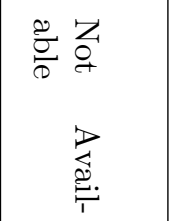 & 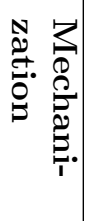 \\
\hline
\end{tabular}




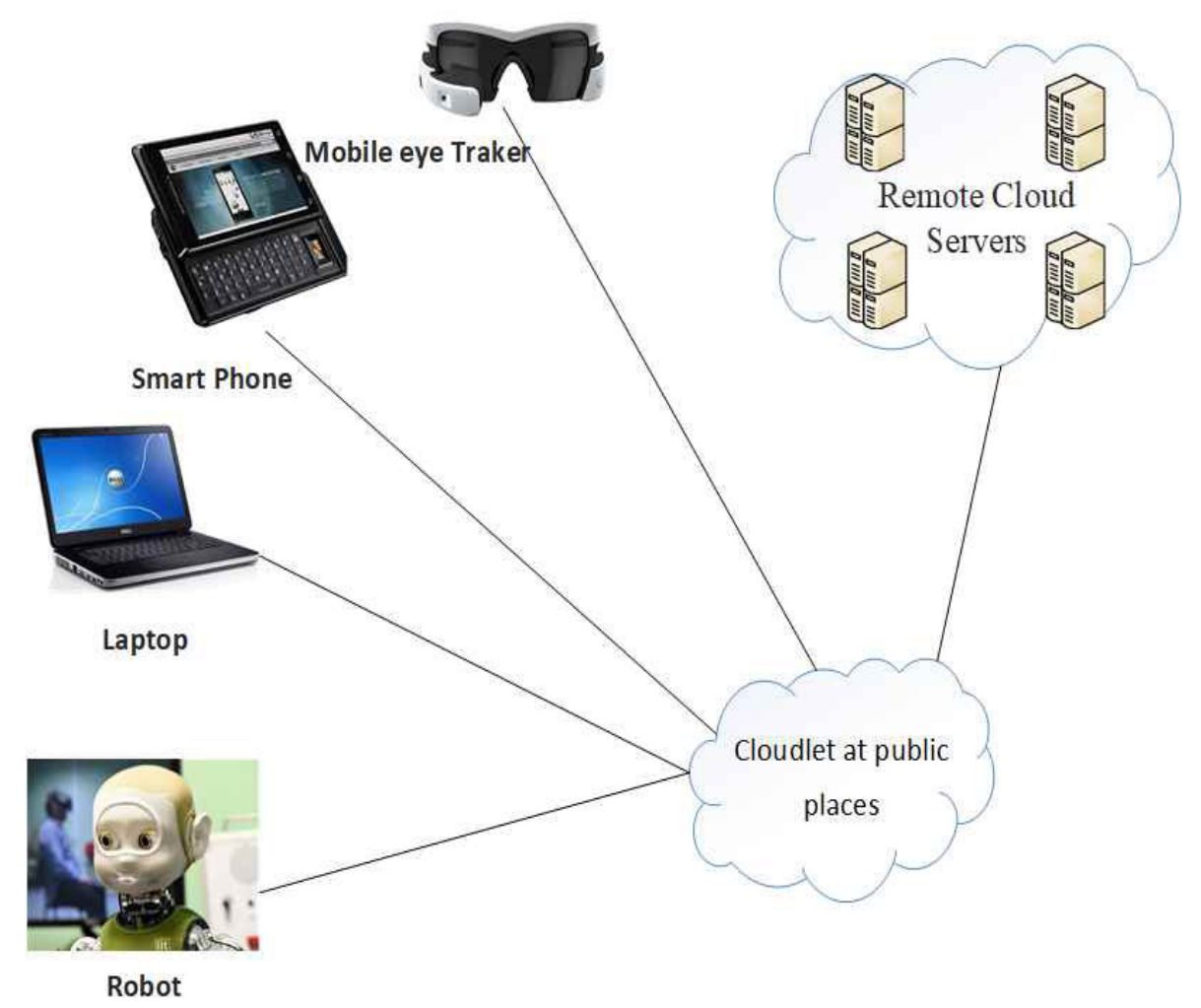

FIG. 4.1. Basic Cloudlet View

Ad-hoc cloudlet can be formed with accumulation of mobile node [36]. This mobile node can join and leave at any point of time. All mobile nodes help to run agent, the agent is responsible for recreating migration and deployment component whenever mobile nodes leave or join. Cloudlet helps to migrate a task from one cloudlet to another cloudlet based on cloudlet configuration and vicinity in case of elastic cloudlet, the mobile nodes are allowed to run on VMs in virtual environment. The node agent can perform dynamic spawing for mobile nodes based on available resources. The concept of elastic cloudlet is comparable with the VM-based cloudlet proposed by Sathyanarayan [22]. It solves the problem of lack of resources by offering pre-configured VM to cloudlet. Elastic cloudlet is formed through public cloud. The only one difference is that makes the both models different with extra layer, which exist in elastic cloudlet to handle mobile user applications.

4.3. Architecture of cloudlet. Cloudlet architecture Fig 4.2 formed with additional layer between mobile devices and cloud. Cloudlets are distributed in geographical area as Wi-Fi access points. The performance of the cloudlet can be calculated based on following three properties:

1. cloudlet size;

2. lifetime of cloudlet node;

3. reachable time.

Cloudlet size. The size of cloudlet is defined based on number of mobile nodes connected to that master node (initiator).

Life-time of cloudlet node. The lifetime of cloudlet node can be calculated based on how much time spent for processing task with initiator node.

Reachable time. The amount of time both mobile node and initiator (master node) in T. the cloudlet architecture can be represented with combination of both Ad-hoc cloudlet and elastic cloudlet. The architecture 


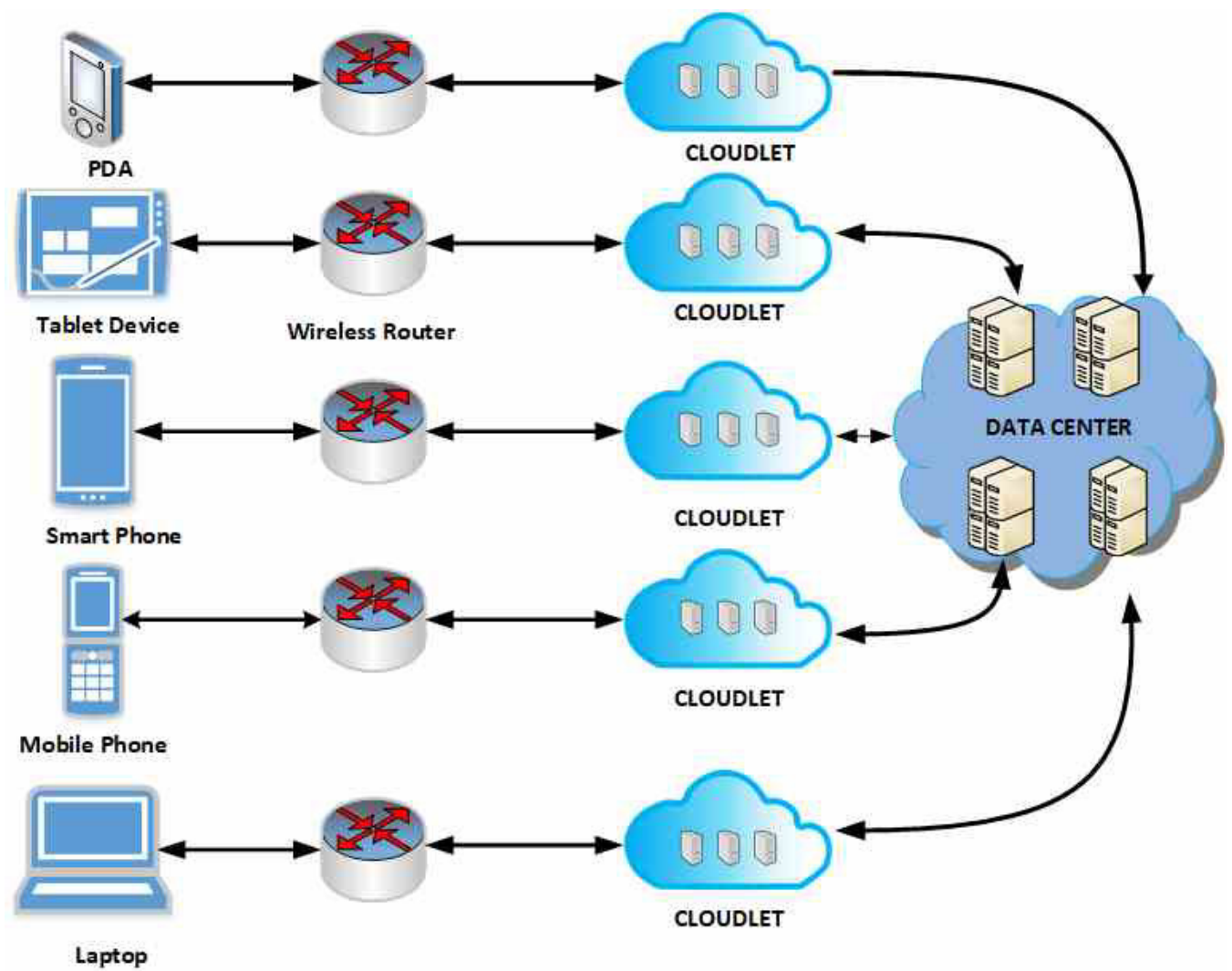

FIG. 4.2. Architecture of cloudlet

discuss about how mobile devices interact and communicate each other.

The architecture is categorized into three layers.

Component layer. In component layer, the number of components (mobile device) together can form deployment environment. Every component is handled by execution environment(EE) which decides whether the component is to run or stop. The components are distributed in area which can be facilitated by employing more than one EE. Component can discover issues related to performance and disclose configuration details to EE. The EE can detect performance issues and provide appropriate solutions such as resource provisioning for offloaded resource hungry tasks.

Node layer. The cloud environment can be formed with no. of servers each server is partitioned by running VMs operating system resides on each Virtual Machine. EE will hold more than one VM for execution. The node can be formed with combination of both hardware and O.S. it is Node Agent [NA] responsibility to manage and monitor all running nodes in cloudlet. NA will also take decision to start or stop any EE. The resource provisioning among all nodes in cloudlet is done by node agent.

Cloudlet layer. The no. of nodes together can be formulated as cloudlet. The cloudlet agent (CA) is responsible to manage all cloudlets and maintain communication with all node agents in cloudlet the node agent of one cloudlet can communication with other cloudlet in order to migrate resource hungry task for execution. The node can be set as cloudlet agent by considering maximum amount resources availability [37].

The following section describes briefly about categories of cloudlet. Fig 4.4 represents categories of cloudlet architecture.

4.3.1. Network Based Architecture. The mobile devices can communicate with nearby cloudlet or other devices with help of network enhance as which is being used among servers in cloud. Mobile devices always connect to near cloudlet via popular networks $3 \mathrm{G}, 4 \mathrm{G}$ and $\mathrm{Wi}$-Fi. The cloudlet distribute computational 


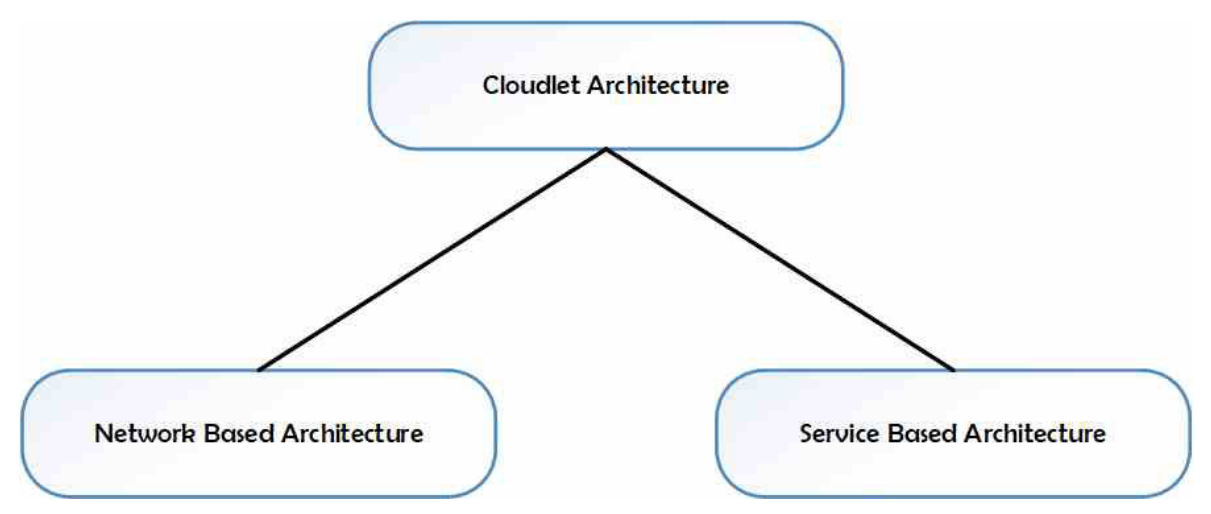

FIG. 4.3. Classification of Cloudlet Architecture

tasks to available cloudlets for executing and send results back to them. The data among cloudlet can be sent and received through routing algorithms. Two popular algorithms can be used to make communication among cloudlet in network i.e. distributed routing algorithm and centralized routing algorithm.

The distributed scheme uses peer-to-peer communication among cloudlets. The cloudlet distributes its present location to all nodes nearby, the node can receive and connect to the cloudlet. The mobile device maintains cloudlet table for storing ID of cloudlet whenever it receives presence of cloudlet information for future use. The cloudlet also maintain mobile table, which stores all mobile IDs that can be connected to cloudlet. The cloudlet broadcast mobile IDs table to other cloudlet to know. Each cloudlet shares mobile node information to ensure to make easy for resource allocation in future.

In centralized scheme, one server is established called as centralized server. The task of the centralized server is to store IDs of all available cloudlet. The broadcasting is done once all cloudlet gets registered with centralized server by sending cloudlet ID whenever the mobile node connects to cloudlet, the cloudlet has to store ID of cloudlet and all attached mobile nodes of cloudlet. It is centralized server task to maintain huge table for storing cloudlet IDs and mobile node IDs. Whenever mobile node wants communicate with other node in another cloudlet, the cloudlet has to send details of cloudlet Id and mobile node Id. The server acts as proxy between cloudlet to send and receive data from one cloudlet to another cloudlet.

4.3.2. Service based architecture. This architecture aims to disclose how data is managed and shared among nodes in cloudlet and among cloudlet as well. The behaviour of service base architecture discussed through following two services.

File editing.. The file can be edited directly in remote cloud otherwise the whole file can be downloaded into local cloudlet where mobile users can edit it. Once the editing is done that file send back to cloud through wireless network. It is cloudlet agent responsibility to maintain synchronization between cloud and cloudlet. When multiple users are allowed to edit file in cloudlet [38].

The steps for file editing in service architecture are:

- The node looks for nearby cloudlet and connect to it after successful connections. The node would calculate the round trip cost from its location.

- After successful connection with remote cloud server request for file editing then, node calculations the round trip cost from its location.

- If the cost of the cloudlet file editing is less than remote cloud file editing, then file editing is done at cloudlet itself, otherwise in remote cloud server.

- The cloudlet update file after successful editing.

Video streaming. The node does video streaming available in remote server by means of cloudlet nodes to save time and energy instead of streaming directly from remote server.

The steps for video streaming in service architecture are:

- The node looks for nearby cloudlet and connects to it. After successful connections, the node calculates round trip cost from its location. 
TABLE 4.1

Mobile Configuration

\begin{tabular}{|c|c|c|}
\hline Year & Memory & Device Type \\
\hline 2018 & 1 TB & High-end devices \\
\hline 2018 & 1 TB & Low-end devices \\
\hline
\end{tabular}

- After successful connection with remote servers the node requests for video streaming and then calculates the round trip cost from its place.

- If the round trip cost of cloudlet is less than remote server then video streaming would take place at cloudlet otherwise from remote server.

- The synchronization concept is not here in video streaming unlike file editing, the video being while downloading into cloudlet.

4.4. Pocket cloudlet. The development of internet has been raising from last few decades by introducing various mobile devices such as smart phones, tablets and other PDAs. The mobile users are able to leverage cloud services by means of advent of internet. The communication channels help to mobile devices to access cloud services. These challenges serves request and bring issues such as energy overhead and latency issues. Two major constrains are mainly raising from radio link i.e. network availability and energy consumption. The mobile communication is not able to serve increasing demands of mobile users the mobile cloud computing provides solutions to address issues. The configuration of mobile devices can be developed in terms of processor and memory size. For example nowadays mobiles are manufactured with extension of memory about 64 GB of non-volatile. The expansion of mobile storage with nominal restrictions can be observed by researches.

Mobile devices having enough storage to store large amount of data locally. The storage availability is specified in Table 4.1. The advent feature hash technology provides more storage irrespective of local storage of mobile device. Most of storage space in mobile devices remain unused. The availability of storage space in mobile device can be used for storing some cloud services locally.

In cloud, specific services are being used often by mobile users. The major usage of specific resources causes data to be downloaded over and over when user download data recursively which causes high latency and more energy consumption. These problems can be solved by means of internal mobile storage to store cloud services which are accessed often. The concept of pocket cloud is formed by storing frequently used data [39]. Pocket cloud can reduce power consumption, high latency and other overhead issues. Storing part of service or entire cloud services into mobile devices. By increasing storage capacity of mobile device, more number of cloud services can be stored in device. The pocket cloud make mobile device more efficient in every possible way.

The pocket cloud provides advantages to mobile users are:

- Pocket cloud enhance user experience by storing cloud services locally.

- Mobile users can access data at any time without delay.

- Pocket cloudlet minimizes burden on cloud servers and radio links in network.

- Since every user have individual mobile device, it is easy to identify usage pattern, storing cloud services in mobile devices on demand basis.

- Security levels has been enhanced for storing sensitive in mobile storage.

The data stored on mobile devices are updated in regular intervals sensitive data is updated frequently on other hand less sensitive data are updated when resource is not constrained i.e. when mobile is getting charged, when network connection is very high. Pocket cloud follows certain protocols before storing services:

- The size of storing data varies each service.

- Security mechanism should be followed before storing data.

- Efficient architecture should be used for storing and retrieving huge amount of data.

- Updating of data by means of proper mechanism on real-time basis.

4.4.1. Pocket cloud architecture (PCA). Pocket cloud architecture Fig 4.4 provides the way in which cloud services are accessed by mobile devices. According to research study, more than $90 \%$ user visit less than 1000 URLs in the specific time period. Cloud services can be transferred from cloud to mobile devices by means 


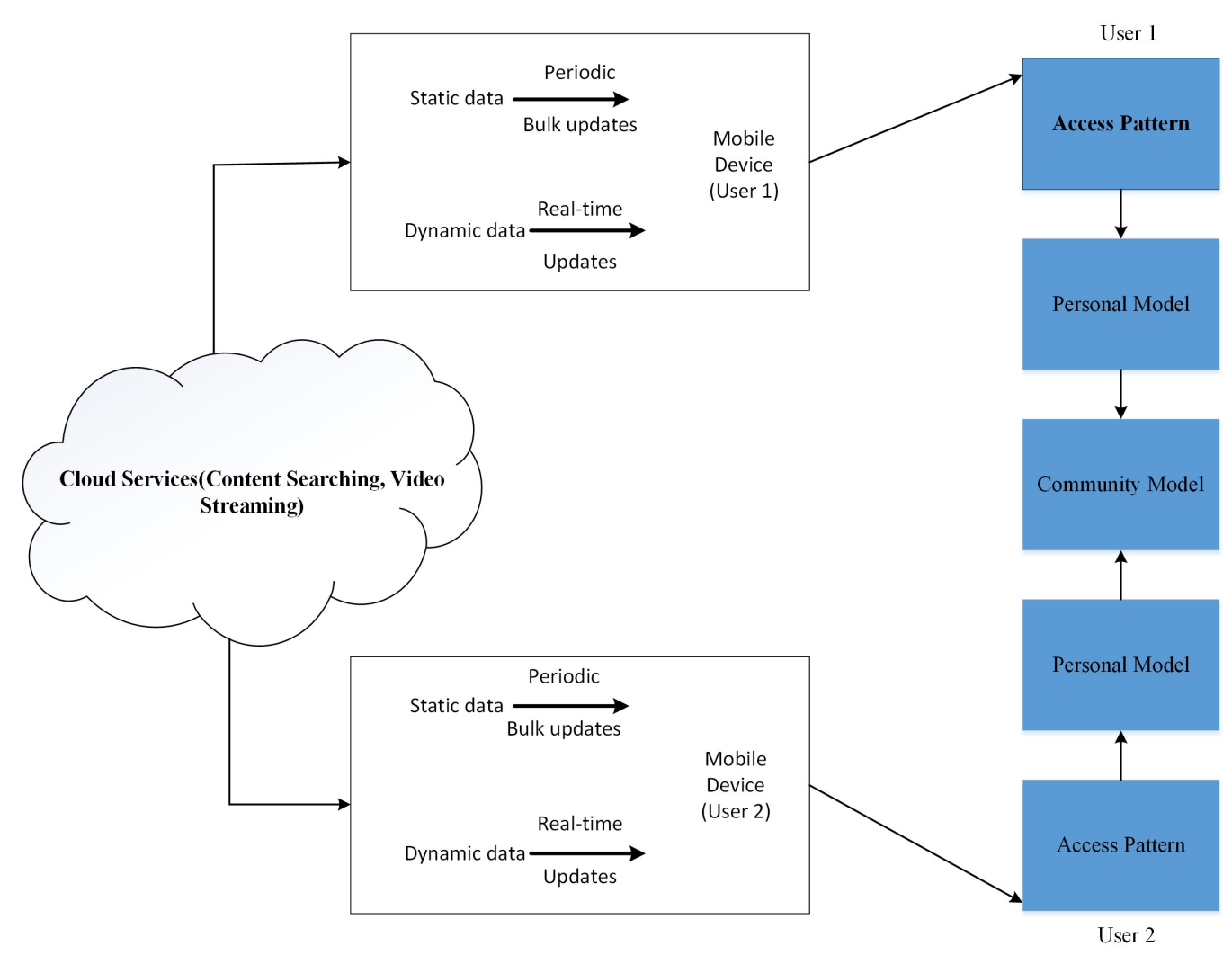

FIG. 4.4. Architecture of PCA

of 3G, $4 \mathrm{G}$ and Wi-Fi long range networks. Mobile devices store data for future use. Data can be classified into two categories static data and dynamic data.

Static data is updated periodically whenever network availability is high with more battery life for example at night time when mobile getting charged. Dynamic data is updated on real-time basis, which require high network availability.

Mobile users access data stored in devices based on patterns which are formed by cloud. These patterns also known as access pattern. Access pattern can be formed as personal model to maximize usage of cloud services. By combining all individual personal models to be formed as community model.

4.5. Comparision between cloud and cloudlet. Many existing research work have mistaken by mentioning that both cloud \& cloudlet are same. But, it is not true, each of them have their own architecture, nature of functioning. There are various parameters to prove both technologies are different paradigms. Table 4.3 shows comparison between cloud and cloudlet.

4.6. Summary of Literature Review on Cloudlet. Modh et al [40] have characterized the idea of blending of two new technologies which are mobile computing and cloud computing into one known as Mobile Cloud Computing (MCC). Mobile Cloud Computing (MCC) helps in providing rich benefits of both the combined technologies. Cloud computing helps to overcome the problem of storage limit as well as increasing the computational power, processing power and storage of various mobile applications. Mobile computing helps in easy access and retrieval of any data stored in our mobile device. Still there are a few difficulties identified with Mobile Cloud Computing. In this paper they have presented different difficulties of systems like Internet availability, data transmission, dormancy, access speed and so on for MCC. They additionally discuss about the one cloudlet solution system for the fundamental system issue of idleness that influences the upgrade of MCC.

As mobile devices are being used widely they are playing an significant role in every individuals life. In any 
case, mobile devices have the limitations, for example, low computational power and quick depletion of power from their batteries. Loai et al in [41], have found the solution that the services of mobile cloud computing can be utilized to run specific assignments at the cloud and send the results to end user devices addition memory and then handles the power. This model of mobile computing is effective with the view of cloudlet scheme. This mobile cloud computing model reduces the expensive technologies such as Wi-Fi, 3G/4G, networks by communicating with the cloudlet directly rather than being in contact with venture cloud server. In addition to this model, a plan which involves the interaction of cloudlet with each other. This plan is certainly known as ace cloudlet administration plan. Colleges, institutions and healthcare centre widely make use of effective Mobile Cloud Computing (MCC) where it necessary to store and access the large amount of information. The MCC model in which the results of non-cloudlet are outperformed is discussed in this model.

Mobile computing is restricted with limitations such as battery, memory and capacity etc. By overwhelming these restrictions the Mobile Cloud Computing has become familiar by offloading the tasks that are beyond the range of end user device capacity to the cloud and later processing the those tasks in cloud are sent back to the user device. Utilization of mobile cloud computing tends to reduce the power consumption and time consumption to process the tasks that are offloaded to the cloud. MCC can be utilized efficiently to reduce the power consumption and time consumption with the help of cloudlet based MCC framework proposed by Jaraweh et al in [42]. Experiment outcomes have demonstrated that utilizing the proposed system lessens the power utilization from the cell phone, in addition to decreasing the correspondence inactivity when the cell phone asks a task to occur remotely while keeping high calibre of administration stander.

Offloading of tasks with high intensity in the mobile into the cloud server by rising innovation mobile cloud computing. Raei et al in [42] proposed analytical based performance model to overcome the problems occurred in expecting results due to the MCC attributes like portability, unsteadiness of $3 \mathrm{G} / \mathrm{Wi}-\mathrm{Fi}$ and virtualization that cannot be predicted. A technique called fixed point iteration technique sets the cyclic reliance between the problematic sub-models. Physical Machine (PM) acts as piece of cloudlet otherwise an open cloud when virtual machine (VM) is maintained on physical machine. This type of MCC is executed based on parameters like network failure and workload. The effects caused due to this parameters are measured based on two measures: request dismissal likelihood and mean reaction delay. This model is understood by the use of SHARPE programming bundle.

Although mobile devices are increasing rapidly in our day to day life. They are limited with certain constraints. Assets in mobile device can be reduced by offloading the high intensity tasks into the nearby cloud with the help of mobile distributed computing. Cloudlet is an essential part for the customer cloud system in focalizing advancement in cloud registering and mobile computing. In this paper, Pang et al [43] exhibits a broad review of examines on cloudlet based. They initially hindsight the development of cloudlet based mobile computing. From that point forward, they audited the current research on the cloudlet based processing offloading and information offloading. Two cases regarding the cloudlet are presented and examined the present scenario, endeavours and upcoming bearings of this field.

According to Dinh et al in [3], MCC has been enlightened with the potential innovation for cloud administrators along with the increase in mobile application and cloud computing idea. Whenever the tasks received by mobile cannot be processed by the mobile, MCC organizes the cloud computing into mobile condition such that cloud computing process the task by overcoming the limitation of mobile device such as battery life, stockpiling and transmission capacity including condition i.e. how heterogeneous, versable and accessible it is and security. We discuss the basic outline of MCC with definition and how it is useful in engineering and its application.

Gai et al [44] stated that utilizing Mobile Cloud Computing (MCC) to empower cloud clients to procure advantages of cloud computing by an ecological amicable technique is an effective procedure for taking care of current modern requests. However, the limitations of remote data transmission and gadget limit have brought different impediments, for example, additional vitality waste and idleness delay, while conveying MCC. A dynamic energy aware cloudlet-based mobile cloud computing model (DECM) has been proposed to overcome the limitation such as additional vitality waste and idleness of remote data transmission and device limit. This model DECM makes use of extra vitality during the interchanging of remote data by dynamic cloudlet-based model (DCL). In this paper, they inspect their model by a recreation of functional situation and give strong outcomes to the assessments. The principle commitments of this paper are twofold. In the first place, this paper 
is the primary investigation in taking care of vitality squander issues inside the dynamic systems administration condition. Second, the proposed display furnishes future research with a rule and hypothetical backings.

According to Sanaei et al in [45], MCC is the resultant of rapid and repeated research exercises that are performed in favour of increasing various mobile devices with the help of different cloud advantages. Encouragement if interoperability, transportability and incorporation between the different stages is important in the middle of such different condition. The facilitators in MCC helps in examining the heterogeneity to understand and also difficulties. The successful MCC undergoes literary struggles when cloud computing and cloud figuring is integrated. In the present paper, we discuss about the characterization of MCC, how to illuminate the important endeavours, testing diversification in figuring. Heterogeneity is classified as equipment, stage, highlight, API and system after the base of heterogeneity is explored. The improvement of cross-stage cloud applications is blocked due to the multi-dimensional heterogeneity in MCC which develops application and code discontinuity issues. Difficulties due to the effects of diversification are recognized through the research and we discuss about the methodologies like virtualization, middleware and service oriented architecture (SOA) is taken care by overcoming heterogeneity.

The Table 4.2 points towards several papers on cloudlet.

\section{Security, Privacy And Challenges In Mobile Cloud Computing (MCC).}

\subsection{Layers of cloud computing.}

Data Centers Layer. It provides hardware facility and infrastructure for cloud. in which, numerous servers are connect via internet to provide services to users [56].

Infrastructure as a Service (IaaS). It provides hardware, storage, servers, networking component for users, and users will pay as you go [56].

Platform as a Service (PaaS). It provides advanced environment for application developing, deploying, and testing [57].

Software as a Service (SaaS). It shares available applications and information remotely via internet with multiple users and pay only for they use [57].

\subsection{Security Breaches And Issues.}

5.2.1. Data ownership. Cloud computing provide facility to user to store purchased data such as video files, audio files, e-books remotely. There can be a chance that user will not be able to access bought data from server and should be aware of access permission of bought data. Mobile cloud computing solve this kind of breaches by using context information like location, capabilities of device, user profile [56].

5.2.2. Privacy. Privacy is one of the significant challenge in mobile cloud computing. Some mobile applications store users personal data in cloud by hiring storage. Third party companies share users sensitive data with government agencies without users permission [45].

5.2.3. Security Issues. Mobile devices are venerable to attacks and chances of stolen data because mobile devices are unprotected. An unauthorized user easily gets access of authorized users. Few security issues mentioned as follows [58]:

- Data loss from loss/stolen devices.

- Information stealing from mobile malware.

- Data leakage happens with untrusted third party.

- Insecure network access and unreliable access points.

- Vulnerabilities with in devices, operating system, design and third party application.

- Near field communication (NFC) proximity based hacking.

The concept of security breach is that the unauthorized user access sensitive data of other user without corresponding user permission. Many organizations treat their data as voluble asset of their company. It is well known fact that ever user knows that it is impossible to avoid loss of data in network world. There are many ways that data could get lost [59]. 
TABLE 4.2

Summary of Literature Review on Cloudlet

\begin{tabular}{|c|c|c|c|c|}
\hline Ref & Cloud & Cloudlet & Problem & Solution \\
\hline$[41]$ & & CL & $\begin{array}{l}\text { Optimizing } \\
\text { power consumption } \\
\text { and high latency. }\end{array}$ & MCCSIM \\
\hline$[42]$ & & CL & $\begin{array}{l}\text { Minimizing } \\
\text { power consumption and latency. }\end{array}$ & $\begin{array}{l}\text { Cloudlet } \\
\text { based MCC }\end{array}$ \\
\hline$[46]$ & & CL & $\begin{array}{l}\text { Optimizing } \\
\text { performance of cloudlet } \\
\text { by considering workload, } \\
\text { recourse capacity, } \\
\text { connection failure rate, } \\
\text { request rejection probability, } \\
\text { mean response delay. }\end{array}$ & $\begin{array}{l}\text { Fixed-point } \\
\text { iteration algorithm }\end{array}$ \\
\hline$[47]$ & & CL & $\begin{array}{l}\text { Optimizing } \\
\text { cloudlet selection and } \\
\text { resource provisioning. }\end{array}$ & $\begin{array}{l}\text { Round } \\
\text { Robin with } \\
\text { load-degree algorithm. }\end{array}$ \\
\hline$[44]$ & $\mathrm{C}$ & CL & $\begin{array}{l}\text { Reducing } \\
\text { power usage for cloud selection. }\end{array}$ & $\begin{array}{l}\text { Dynamic } \\
\text { cloudlet } \\
\text { selection model }\end{array}$ \\
\hline$[48]$ & $\mathrm{C}$ & CL & $\begin{array}{l}\text { Developing } \\
\text { hybrid application by } \\
\text { optimizing power and } \\
\text { latency issues. }\end{array}$ & $\begin{array}{l}\text { Automation } \\
\text { Script with } \\
\text { Exhaustive Search algorithm }\end{array}$ \\
\hline$[49]$ & $\mathrm{C}$ & CL & $\begin{array}{l}\text { Cloudlet } \\
\text { selection and processing } \\
\text { with low power consumption } \\
\text { and latency . }\end{array}$ & $\begin{array}{l}\text { Recourse } \\
\text { allocation using centralized } \\
\text { proxy server. }\end{array}$ \\
\hline$[50]$ & $\mathrm{C}$ & CL & $\begin{array}{l}\text { Optimizing } \\
\text { power consumption and latency } \\
\text { by distributing tasks } \\
\text { among cloudlets. }\end{array}$ & $\begin{array}{l}\text { MILP } \\
\text { linear programming model }\end{array}$ \\
\hline$[51]$ & $\mathrm{C}$ & CL & $\begin{array}{l}\text { Optimizing } \\
\text { bandwidth and resource } \\
\text { in cloudlet based MCC. }\end{array}$ & $\begin{array}{l}\text { triple-stage Stackelberg game } \\
\text { using backward } \\
\text { method. }\end{array}$ \\
\hline$[52]$ & $\mathrm{C}$ & CL & $\begin{array}{l}\text { Minimizing } \\
\text { CPU execution time } \\
\text { and memory usage. }\end{array}$ & $\begin{array}{l}\text { Bee's } \\
\text { life algorithm }\end{array}$ \\
\hline$[53]$ & & CL & $\begin{array}{l}\text { Load } \\
\text { balancing among fog nodes } \\
\text { to optimize power and recourse } \\
\text { usage. }\end{array}$ & $\begin{array}{l}\text { Optimal } \\
\text { Multi-User } \\
\text { Small Cell Clustering }\end{array}$ \\
\hline$[54]$ & & CL & $\begin{array}{l}\text { Optimizing } \\
\text { user access mode selection. }\end{array}$ & $\begin{array}{l}\text { Evolution } \\
\text { Algorithm. }\end{array}$ \\
\hline$[55]$ & & CL & $\begin{array}{l}\text { Distribution } \\
\text { of load among nodes } \\
\text { to least latency. }\end{array}$ & $\begin{array}{l}\text { Matching } \\
\text { theory. }\end{array}$ \\
\hline
\end{tabular}


TABLE 4.3

Comparision Between Cloud computing and cloudlet

\begin{tabular}{|c|c|c|}
\hline Parameters & Cloud Computing(CC) & Cloudlet \\
\hline State & Hard and Soft State & soft-state \\
\hline Management & $\begin{array}{c}\text { Professionally } \\
\text { Managed }\end{array}$ & Self Managed \\
\hline Large space \\
$\begin{array}{c}\text { Required } \\
\text { for maintaining } \\
\text { servers }\end{array}$ & Established at organization \\
\hline Networship & $\begin{array}{c}\text { Centralized } \\
\text { Management }\end{array}$ & Decentralized Management \\
\hline Sharing & Internet & LAN \\
\hline cost & $\begin{array}{c}\text { Unlimited devices } \\
\text { Communicate and } \\
\text { share data }\end{array}$ & $\begin{array}{c}\text { Limited Devices only } \\
\text { share data and } \\
\text { communicate }\end{array}$ \\
\hline security & More secure and Reliable & Investment is Low \\
\hline
\end{tabular}

\subsection{Mobile cloud computing suffers from following risks.}

- User does not know where exactly mobile data is stored in mobile cloud computing environment which leads user does not have control over stored data.

- Physical damage of cloud server, loss of encoding key and due to malicious insider, risk of data loss may arise.

- Customer may intentionally plant virus of phishing attack in to cloud server which may lead to loss of other users data, and cloud provide is unable to do anything because violation of privacy policy of company.

- When cloud provider services number of users, flaw in encryption may lead to unauthorized encryption.

- As per service level agreement cloud provider should maintain security level Security risk may rise in Iaas due to lack of isolation among hosted virtual machine in single server.

- Most users share their sensitive and personal data through mobile application for instance online transaction that can be attacker main target.

5.4. Security Issues of Mobile Cloud Computing. This section describe different possible attacks in mobile cloud computing.

SQL Injection Attack. The attacker adds malicious code in standard SQL so that attacker get unauthorized access of database, is able to access sensitive data [60].

Browser Security. Every user use browser to transmit data over network. Browser uses SSL technology to provide protection to user authentication details. But attacker always tries to break user credentials by using sniffing package which is installed on intermediary host.

Denial of service. The attacker prevents user accessing services from cloud [61].

Cookie poisoning. The attacker changes content of cookie to have illegal access of application [62].

Flooding attacks. Attacker continuously sends resource required request to cloud server so that cloud get flooded with ample requests. cloud has feature called scalability based on number of requests given send by users but intruder stop server from serving actual users by sending requests rapidly [63].

Incomplete data deletion. When data is deleted, it does not remove copy of that data from backup server until the operation system of the server is commanded specially by network service provider. Precise data deletion is impossible because replicas stored in backup server [63].

Usually user is able to connect cloud server by using web browser or web services [70]. Web service attacks also effect cloud computing. In spite of cloud security uses XML signature for protecting an element name, attribute, value from attackers. 
A Survey on Mobile Cloud Computing: Mobile Computing + Cloud Computing $(\mathrm{MCC}=\mathrm{MC}+\mathrm{CC})$

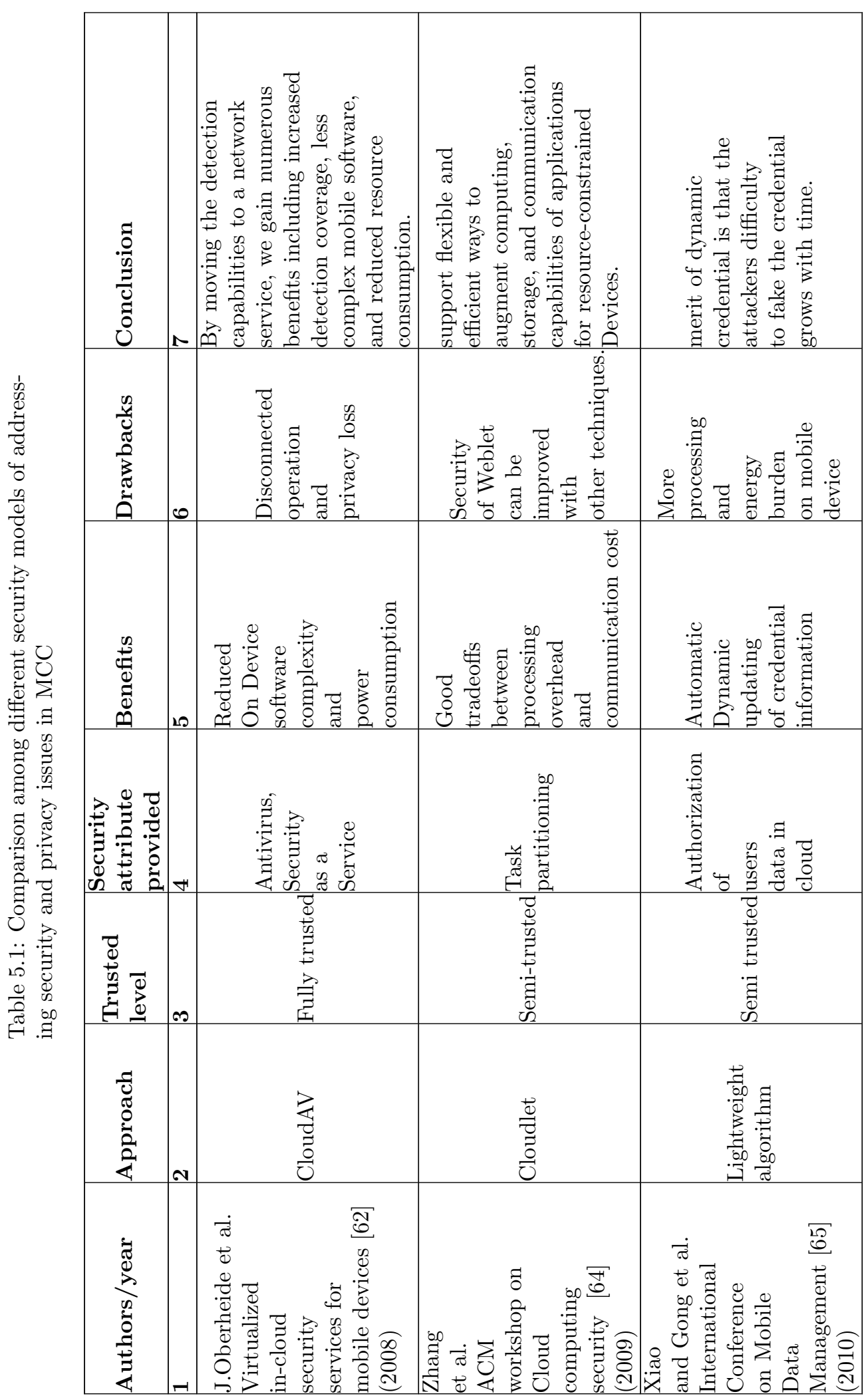




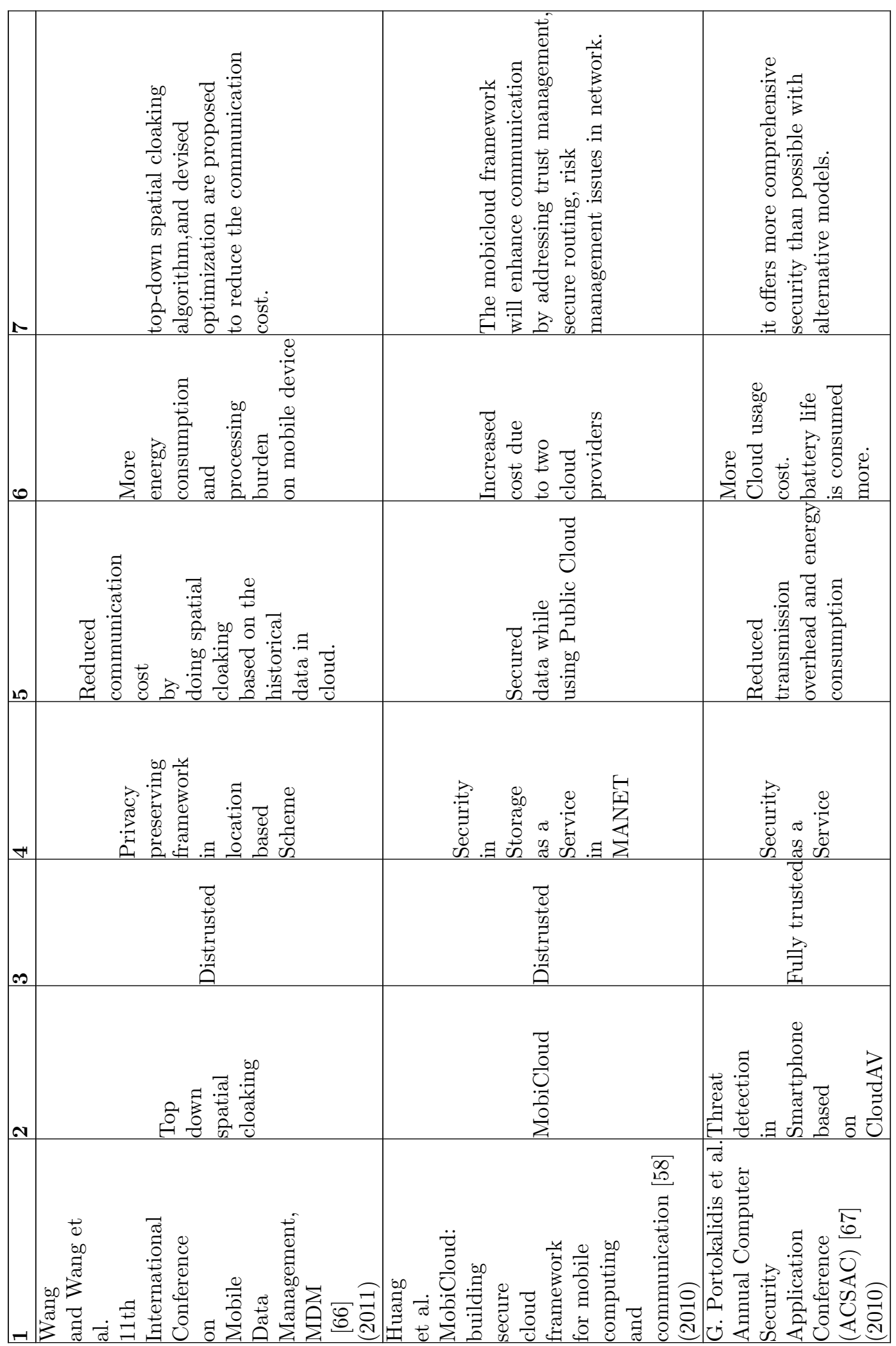




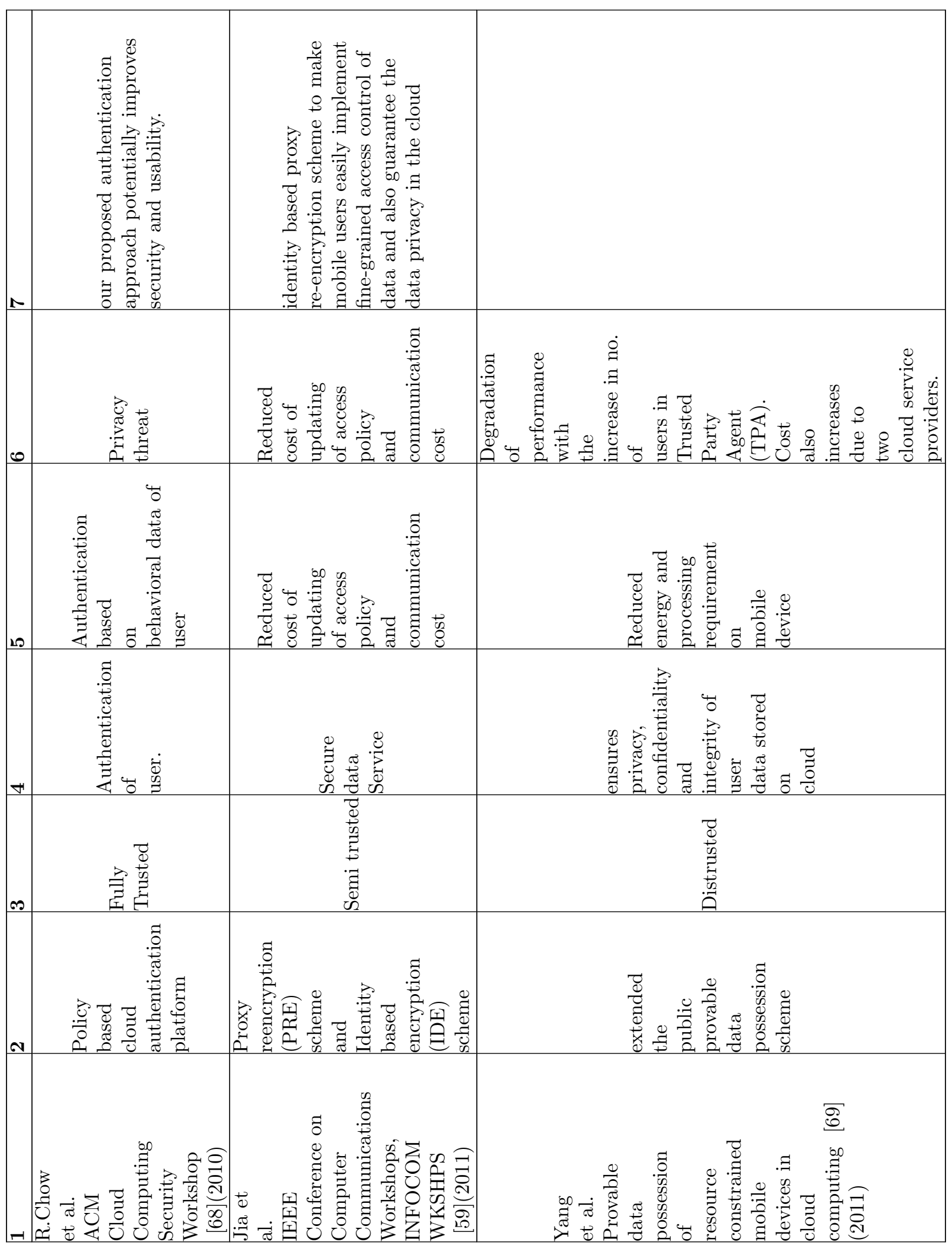







6. Conclusion. In this article, we have discussed about various concepts in mobile cloud computing: (1) Mobile computing, (2) cloud computing, (3) mobile cloud computing,(4) offloading approach, (5) cloudlet approach and (6)security and privacy. We have given extensive survey on existing frameworks on computation offloading. Even through, there are various frameworks presented, but the objective of each framework is to improving mobile performance by reducing energy consumption and response time.

We have provided a survey on emerging cloudlet technology and challenges. the concept of cloudlet aims to address high latency and response time issues by brining cloud recourses closer to mobile user. We have analyzed comparison among popular cloudlet architecture and selection techniques; the secure routing protocol used to protect communication channel between devices and cloud as well as need to be addressed several issues such as data integrity, authentication, authorization and access control. We believe that by introducing edge computing architecture for optimizing mobile performance in MCC will take place in near feature.

\section{REFERENCES}

[1] R. Meulen, "Gartner Says Global Smartphone Sales to Only Grow 7 Per Cent in 2016."

[2] B. Butler, "Gartner: Cloud Putting Crimp in Traditional Software, Hardware Sales," Network World, 2012.

[3] H. T. Dinh, C. Lee, D. Niyato, and P. Wang, "A survey of mobile cloud computing: architecture, applications, and approaches," Wireless communications and mobile computing, vol. 13, no. 18, pp. 1587-1611, 2013.

[4] M. Tulloch, Introducing Windows Azure for IT Professionals. Microsoft Press, 2013.

[5] R. Buyya, "Introduction to the ieee transactions on cloud computing," IEEE Transactions on Cloud Computing, vol. 1, no. 1, pp. 3-21, 2013.

[6] P. Mell, T. Grance, and Others, "The NIST definition of cloud computing," 2011.

[7] C. V. N. Index, "Global mobile data traffic forecast update 2014-2019 white paper, feb 2015," See: http://www. cisco. $\mathrm{com} / \mathrm{c} / \mathrm{en} / \mathrm{us} /$ solutions/collateral/service-provider/visual-networking-index-vni/white_paper_c11-520862. html, 2015.

[8] L. Yang, J. Cao, Y. Yuan, T. Li, A. Han, and A. Chan, "A framework for partitioning and execution of data stream applications in mobile cloud computing," ACM SIGMETRICS Performance Evaluation Review, vol. 40, no. 4, pp. 23-32, 2013.

[9] A. Klein, C. Mannweiler, J. Schneider, and H. D. Schotten, "Access schemes for mobile cloud computing," in Mobile Data Management (MDM), 2010 Eleventh International Conference on. IEEE, 2010, pp. 387-392.

[10] P. Bahl, R. Y. Han, L. E. Li, and M. Satyanarayanan, "Advancing the state of mobile cloud computing," in Proceedings of the third ACM workshop on Mobile cloud computing and services. ACM, 2012, pp. 21-28.

[11] E. Miluzzo, R. Cáceres, and Y.-F. Chen, "Vision: mClouds-computing on clouds of mobile devices," in Proceedings of the third ACM workshop on Mobile cloud computing and services. ACM, 2012, pp. 9-14.

[12] R. Kamal, Mobile Computing. Oxford University Press, Inc., 2008.

[13] L. S. Ashiho, "Mobile technology: Evolution from 1G to 4G," Electronics for you, pp. 94-98, 2003.

[14] M. Armbrust, A. Fox, R. Griffith, A. D. Joseph, R. Katz, A. Konwinski, G. Lee, D. Patterson, A. Rabkin, I. Stoica, and Others, "A view of cloud computing," Communications of the ACM, vol. 53, no. 4, pp. 50-58, 2010.

[15] R. Buyya, J. Broberg, and A. M. Goscinski, Cloud computing: Principles and paradigms. John Wiley \& Sons, 2010, vol. 87.

[16] D. Durkee, "Why cloud computing will never be free," Queue, vol. 8, no. 4, p. 20, 2010.

[17] M. D. Dikaiakos, D. Katsaros, P. Mehra, G. Pallis, and A. Vakali, "Cloud computing: Distributed internet computing for IT and scientific research," IEEE Internet computing, vol. 13, no. 5, 2009.

[18] K. Chard, S. Caton, O. Rana, and K. Bubendorfer, "Social cloud: Cloud computing in social networks," in Cloud Computing (CLOUD), 2010 IEEE 3rd International Conference on. IEEE, 2010, pp. 99-106.

[19] B.-G. Chun, S. Ihm, P. Maniatis, M. Naik, and A. Patti, "Clonecloud: elastic execution between mobile device and cloud," in Proceedings of the sixth conference on Computer systems. ACM, 2011, pp. 301-314.

[20] K. Akherfi, M. Gerndt, and H. Harroud, "Mobile cloud computing for computation offloading: Issues and challenges," Applied Computing and Informatics, 2016.

[21] E. Cuervo, A. Balasubramanian, D.-k. Cho, A. Wolman, S. Saroiu, R. Chandra, and P. Bahl, "MAUI: making smartphones last longer with code offload," in Proceedings of the 8th international conference on Mobile systems, applications, and services. ACM, 2010, pp. 49-62.

[22] M. Satyanarayanan, P. Bahl, R. Caceres, and N. Davies, "The case for vm-based cloudlets in mobile computing," IEEE pervasive Computing, vol. 8, no. 4, 2009.

[23] H. Qian and D. Andresen, "Jade: Reducing energy consumption of android app," the International Journal of Networked and Distributed Computing (IJNDC), Atlantis press, vol. 3, no. 3, pp. 150-158, 2015.

[24] B. Zhao, Z. Xu, C. Chi, S. Zhu, and G. Cao, "Mirroring smartphones for good: A feasibility study," in International Conference on Mobile and Ubiquitous Systems: Computing, Networking, and Services. Springer, 2010 , pp. 26-38.

[25] R. Kemp, N. Palmer, T. Kielmann, and H. Bal, "Cuckoo: a computation offloading framework for smartphones," in International Conference on Mobile Computing, Applications, and Services. Springer, 2010, pp. 59-79.

[26] F. Xia, F. Ding, J. Li, X. Kong, L. T. Yang, and J. Ma, "Phone2Cloud: Exploiting computation offloading for energy saving on smartphones in mobile cloud computing," Information Systems Frontiers, vol. 16, no. 1, pp. 95-111, 2014.

[27] S. Kosta, A. Aucinas, P. Hui, R. Mortier, and X. Zhang, "Thinkair: Dynamic resource allocation and parallel execution in the cloud for mobile code offloading," in Infocom, 2012 Proceedings IEEE. IEEE, 2012, pp. 945-953. 
[28] T. Verbelen, P. Simoens, F. De Turck, and B. Dhoedt, "Cloudlets: Bringing the cloud to the mobile user," in Proceedings of the third ACM workshop on Mobile cloud computing and services. ACM, 2012, pp. 29-36.

[29] M. Satyanarayanan, Z. Chen, K. Ha, W. Hu, W. Richter, and P. Pillai, "Cloudlets: at the leading edge of mobile-cloud convergence," in Mobile Computing, Applications and Services (MobiCASE), 2014 6th International Conference on. IEEE, 2014, pp. 1-9.

[30] S. Simanta, K. Ha, G. Lewis, E. Morris, and M. Satyanarayanan, "A reference architecture for mobile code offload in hostile environments," in International Conference on Mobile Computing, Applications, and Services. Springer, 2012, pp. $274-293$.

[31] S. Clinch, J. Harkes, A. Friday, N. Davies, and M. Satyanarayanan, "How close is close enough? Understanding the role of cloudlets in supporting display appropriation by mobile users," in Pervasive Computing and Communications (PerCom), 2012 IEEE International Conference on. IEEE, 2012, pp. 122-127.

[32] Z. Dou, "Benefits of utilizing an edge server (cloudlet) in the mocha architecture," Ph.D. dissertation, University of Rochester. Department of Electrical and Computer Engineering, 2013.

[33] D. Fesehaye, Y. Gao, K. Nahrstedt, and G. Wang, "Impact of cloudlets on interactive mobile cloud applications," in Enterprise Distributed Object Computing Conference (EDOC), 2012 IEEE 16th International. IEEE, 2012, pp. 123-132.

[34] Y. Li and W. Wang, "Can mobile cloudlets support mobile applications?" in Infocom, 2014 proceedings ieee. IEEE, 2014, pp. 1060-1068.

[35] J. Flinn and M. Satyanarayanan, Energy-aware adaptation for mobile applications. ACM, 1999, vol. 33, no. 5.

[36] Y.-C. Tseng, S.-Y. Ni, Y.-S. Chen, and J.-P. Sheu, "The broadcast storm problem in a mobile ad hoc network," Wireless networks, vol. 8, no. 2-3, pp. 153-167, 2002.

[37] H. Wang, "Accelerating mobile-cloud computing using a cloudlet," Ph.D. dissertation, University of Rochester. Department of Electrical and Computer Engineering, 2013.

[38] J. Wu and H. Li, "On calculating connected dominating set for efficient routing in ad hoc wireless networks," in Proceedings of the 3rd international workshop on Discrete algorithms and methods for mobile computing and communications. ACM, 1999, pp. 7-14.

[39] E. Koukoumidis, D. Lymberopoulos, K. Strauss, J. Liu, and D. Burger, "Pocket cloudlets," in ACM SIGPLAN Notices, vol. 46, no. 3. ACM, 2011, pp. 171-184.

[40] R. M. Modh and J. M. Patel, "A study on improving challenges of network with cloudlets for mobile cloud computing," International Journal of Global Research in Computer Science (UGC Approved Journal), vol. 4, no. 4, pp. 143-146, 2013.

[41] A. T. Lo'ai, W. Bakheder, and H. Song, "A mobile cloud computing model using the cloudlet scheme for big data applications," in Connected Health: Applications, Systems and Engineering Technologies (CHASE), 2016 IEEE First International Conference on, 2016, pp. 73-77.

[42] Y. Jararweh, L. Tawalbeh, F. Ababneh, and F. Dosari, "Resource efficient mobile computing using cloudlet infrastructure," in Mobile Ad-hoc and Sensor Networks (MSN), 2013 IEEE Ninth International Conference on. IEEE, 2013, pp. 373-377.

[43] Z. Pang, L. Sun, Z. Wang, E. Tian, and S. Yang, "A survey of cloudlet based mobile computing," in Cloud Computing and Big Data (CCBD), 2015 International Conference on. IEEE, 2015, pp. 268-275.

[44] K. Gai, M. Qiu, H. Zhao, L. Tao, and Z. Zong, "Dynamic energy-aware cloudlet-based mobile cloud computing model for green computing," Journal of Network and Computer Applications, vol. 59, pp. 46-54, 2016.

[45] Z. Sanaei, S. Abolfazli, A. Gani, and R. Buyya, "Heterogeneity in mobile cloud computing: taxonomy and open challenges," IEEE Communications Surveys \& Tutorials, vol. 16, no. 1, pp. 369-392, 2014.

[46] H. Raei, N. Yazdani, and R. Shojaee, "Modeling and performance analysis of cloudlet in Mobile Cloud Computing," Performance Evaluation, vol. 107, pp. 34-53, 2017.

[47] R. Somula and R. Sasikala, "Round robin with load degree: An algorithm for optimal cloudlet discovery in mobile cloud computing," Scalable Computing: Practice and Experience, vol. 19, no. 1, pp. 39-52, 2018.

[48] A. Akbar and P. R. Lewis, "Towards the optimization of power and bandwidth consumption in mobile-cloud hybrid applications," in Fog and Mobile Edge Computing (FMEC), 2017 Second International Conference on. IEEE, 2017, pp. 213-218.

[49] A. Mukherjee, D. De, and D. G. Roy, "A power and latency aware cloudlet selection strategy for multi-cloudlet environment," IEEE Transactions on Cloud Computing, 2016.

[50] Y. Jararweh, M. Al-Ayyoub, M. Al-Quraan, A. T. Loai, and E. Benkhelifa, "Delay-aware power optimization model for mobile edge computing systems," Personal and Ubiquitous Computing, vol. 21, no. 6, pp. 1067-1077, 2017.

[51] S. Meng, Y. Wang, Z. Miao, and K. Sun, "Joint optimization of wireless bandwidth and computing resource in cloudlet-based mobile cloud computing environment," Peer-to-Peer Networking and Applications, vol. 11, no. 3, pp. 462-472, 2018.

[52] S. Bitam, S. Zeadally, and A. Mellouk, "Fog computing job scheduling optimization based on bees swarm," Enterprise Information Systems, vol. 12, no. 4, pp. 373-397, 2018.

[53] J. Oueis, E. C. Strinati, and S. Barbarossa, "The fog balancing: Load distribution for small cell cloud computing," in Vehicular Technology Conference (VTC Spring), 2015 IEEE 81st. IEEE, 2015, pp. 1-6.

[54] S. Yan, M. Peng, M. A. Abana, and W. Wang, "An evolutionary game for user access mode selection in fog radio access networks," IEEE Access, vol. 5, pp. 2200-2210, 2017.

[55] M. S. Elbamby, M. Bennis, and W. Saad, "Proactive edge computing in latency-constrained fog networks," in Networks and Communications (EuCNC), 2017 European Conference on. IEEE, 2017, pp. 1-6.

[56] H. Wang, S. Wu, M. Chen, and W. Wang, "Security protection between users and the mobile media cloud," IEEE Communications Magazine, vol. 52, no. 3, pp. 73-79, 2014.

[57] D. Dev and K. L. Baishnab, "Notice of Violation of IEEE Publication Principles A Review and Research Towards Mobile 
Cloud Computing," in Mobile Cloud Computing, Services, and Engineering (MobileCloud), 2014 2nd IEEE International Conference on. IEEE, 2014, pp. 252-256.

[58] D. Huang, X. Zhang, M. Kang, and J. Luo, "MobiCloud: building secure cloud framework for mobile computing and communication," in Service Oriented System Engineering (SOSE), 2010 Fifth IEEE International Symposium on. Ieee, 2010, pp. $27-34$.

[59] W. Jia, H. Zhu, Z. Cao, L. Wei, and X. Lin, "SDSM: a secure data service mechanism in mobile cloud computing," in Computer Communications Workshops (INFOCOM WKSHPS), 2011 IEEE Conference on. IEEE, 2011, pp. 1060-1065.

[60] P. Garg and V. Sharma, "An efficient and secure data storage in Mobile Cloud Computing through RSA and Hash function," in Issues and Challenges in Intelligent Computing Techniques (ICICT), 2014 International Conference on. IEEE, 2014, pp. 334-339.

[61] Q. Yan, F. R. Yu, Q. Gong, and J. Li, "Software-defined networking (SDN) and distributed denial of service (DDoS) attacks in cloud computing environments: A survey, some research issues, and challenges," IEEE Communications Surveys E6 Tutorials, vol. 18, no. 1, pp. 602-622, 2016.

[62] J. Oberheide, K. Veeraraghavan, E. Cooke, J. Flinn, and F. Jahanian, "Virtualized in-cloud security services for mobile devices," in Proceedings of the first workshop on virtualization in mobile computing. ACM, 2008, pp. 31-35.

[63] S. Zonouz, A. Houmansadr, R. Berthier, N. Borisov, and W. Sanders, "Secloud: A cloud-based comprehensive and lightweight security solution for smartphones," Computers \& Security, vol. 37, pp. 215-227, 2013.

[64] X. Zhang, J. Schiffman, S. Gibbs, A. Kunjithapatham, and S. Jeong, "Securing elastic applications on mobile devices for cloud computing," in Proceedings of the 2009 ACM workshop on Cloud computing security. ACM, 2009, pp. 127-134.

[65] S. Xiao and W. Gong, "Mobility can help: protect user identity with dynamic credential," in Mobile Data Management (MDM), 2010 Eleventh International Conference on. IEEE, 2010, pp. 378-380.

[66] S. Wang and X. S. Wang, "In-device spatial cloaking for mobile user privacy assisted by the cloud," in Mobile Data Management (MDM), 2010 Eleventh International Conference on. IEEE, 2010, pp. 381-386.

[67] G. Portokalidis, P. Homburg, K. Anagnostakis, and H. Bos, "Paranoid android: versatile protection for smartphones," in Proceedings of the 26th Annual Computer Security Applications Conference. ACM, 2010, pp. 347-356.

[68] R. Chow, M. Jakobsson, R. Masuoka, J. Molina, Y. Niu, E. Shi, and Z. Song, "Authentication in the clouds: a framework and its application to mobile users," in Proceedings of the 2010 ACM workshop on Cloud computing security workshop. ACM, 2010, pp. 1-6.

[69] J. Yang, H. Wang, J. Wang, C. Tan, and D. Yu, "Provable data possession of resource-constrained mobile devices in cloud computing." JNW, vol. 6, no. 7, pp. 1033-1040, 2011.

[70] V. Varadharajan and U. Tupakula, "Security as a service model for cloud environment," ieee transactions on network and service management, vol. 11, no. 1, pp. 60-75, 2014.

Edited by: Rajkumar Rajasekaran

Received: Jun 29, 2018

Accepted: Oct 25, 2018 



\title{
EXECUTION ANALYSIS OF SPATIAL DATA STORAGE INDEXING ON CLOUD ENVIRONMENT
}

\author{
KARTHI $\mathrm{S}^{*}$ AND PRABU $\mathrm{S}^{\dagger}$
}

\begin{abstract}
Cloud computing overcome the GIS issues are huge storage, computing and reliability. Cloud computing with SpatialHadoop framework gives high performance in GIS. This paper presents spatial partition, global index and map reduce operations were studied and described in detail. Bloom filter R-tree index in the Map-reduce for providing more efficiency than the existing approaches. The BR-tree index on Map-Reduce is implemented in SpatialHadoop process that reduces intermediate data access time. Global index decreases the number of data accesses for range queries and thus improves efficiency. It is observed through experimental results that the proposed index along cloud environment performs better than existing techniques.
\end{abstract}

Key words: Spatialhadoop, BR-tree Index, Global Index, Map Reduce, Cloud Computing

AMS subject classifications. 68M14, 97R50

1. Introduction. Geographical Information System (GIS) presently we have accumulated huge geospatial data which are expanding and updating every day. GIS is utilized to catch, store, and process, analyze and display the present geospatial data. A fruitful GIS stage is not just ready to deal with vast data with complex properties, additionally gives huge data process and execution, and other computational issues. The GI [26] confront challenges in computing intensity, data intensity and concurrent access intensity. These difficulties require the preparation of a figuring framework that can better bolster revelation, give scalable and concurrent access. The cloud environment gives possible and flexible solution for the GIS issues.

A Spatial database remains as databank, and it is enhanced to keep and question information this is associated with objects in area, which includes factors, traces, polygons etc.... Though normal databases is detain to diverse numeric and personality kinds of statistics, supplementary functionality desires to be delivered for records to system latitudinal statistics types. Spatial facts stand the numerical connection between people, region, and activities. This facts can explicitly illustrate what's taking place (in which, why and how) to show the perception and effect of the beyond, the present and the (probable) destiny [1]. The proliferation of cellular programs and the huge of hardware sensing devices boom the streamed information towards the web hosting statistics-centers. This boom reasons a flooding of records. Taking blessings from these big dataset stores is a key point in growing deep insights for analysts for you to beautify gadget productiveness and to capture new commercial enterprise opportunities. Spatial analysis the crux of GIS as it includes all the alterations, manipulations, and strategies that may be carried out to geographic statistics to add fee to them, to aid decisions, and to show patterns and anomalies that aren't at once obvious. Spatial evaluation is the technique via which we flip uncooked data into beneficial information. The time period analytical cartography is now and again used to refer to methods of analysis that may be carried out to maps to make them more beneficial and informative. There are masses of approaches spatial facts can help and aide in the regular lives of all of us. The utilization of spatial information is indexed as underneath:

1. Satellite pix deliver daily weather reviews and provide farmers with facts for precision agriculture

2. Convert the integral to a linear combination of integrals of products of B-splines and provide a recurrence for integrating the product of a pair of B-splines.

3. Airborne infra-red scanners tune our bushfires

4. Ambulance message services

5. Global positioning structures divulge the region of hundreds of vans and taxis

6. Real estate income use geographic records systems

7. All styles of mapping.

The spatial information enterprise is a component of the broader facts technology sector [3] and has scientific and technical hyperlinks to all different disciplines along with environmental technology, engineering, pc technology,

* School of Computer Science and Engineering, VIT University, Vellore, TN, India

${ }^{\dagger}$ School of Computer Science and Engineering, VIT University, Vellore, TN, India 
fitness shipping, logistics, planning, useful resource control and electronics.

1.1. Data categories in spatial warehouse. The maximum recycled the classification to signify the issues of spatial enquiry consider three sorts of statistics:

Points or Events Occurrences expressed via single value identified as factors in zone, denominated theme tactics. Some samples are: sickness occurrences, crime spots, and the localization of botanical species.

Constant surfaces predicted can be beginning a hard and fast of vicinity examples which are regularly or erratically disbursed. Typically, this kind of statistics consequences from herbal sources studies, which incorporates topographical, biological, phytogeography, environmental and pedagogical charts. Zones with Calculations and Aggregation Tolls - it includes information linked to population studies, like census and fitness information [10], and which is probably initially mentioned to people located in precise elements in universe. For secrecy motives those information are aggregated in analysis gadgets, usually enclosed thru locked polygons (postal addressing zones, census tracts, municipalities). The symmetrical representations used consist of the subsequent alternatives: 2D Points: It is a well-ordered pair of $\mathrm{x}, \mathrm{y}$ values of three-dimensional directs. A factor suggests that vicinity of incidence of an occasion, like in the case of mortality via the use of outside causes. Polygons: It is a hard and fast of methodical pairs values that indicates $\mathrm{x}, \mathrm{y}$ values of three-dimensional directs, in this type of method that the ultimate opinion is same to the prime for this reason forming closed vicinity within the aircraft. In the most effective situation, every polygon delimits a person item within the most famous case; a man or woman area of interest may be delimited via numerous polygons $[11,14,15]$. Samples: encompass with ordered pairs of $\mathrm{x}, \mathrm{y}$, and $\mathrm{z}$ values in which contains the $\mathrm{x}, \mathrm{y}$ pairs mean the physical coordinates and then $\mathrm{z}$ suggests that the price of the premeditated singularity aimed at that location of zones. Typically the examples are related to area of studies, collectively with geochemical, oceanographic and then geo-physical records. The idea of a example can be widespread to the event of numerous dimensions on the identical area. Regular Grid: It is an environment in which every part is related towards a numeric rate. In this matrix, we can calculate the points which associated with a place on the ground floor. Preliminary from a initial coordinates typically noted the inferior left corner of the 10X30 matrix, and using spacing in equally to the straight and vertical directions. Image: It is in the form of matrix in which every detail is related to a numeral value (commonly from 0 to 255 variety), recycled for conception [14]. This type of condition is used to the photo presentation of a normal grid. The statistical standards in the network are ascended in shape with the production variety of the image; the superior values stand to be proven in sunnier gray shades, and then decrease in duskier gray qualities. Maximum of the geographical information systems provide the ability of providing a fixed grid in the system of a picture (popular shades or in black \& white), through a conversion that may remain computerized or measured via purchaser. Three essential shapes of spatial facts is proven in Fig. 1.1.

Database systems exercise directories to unexpectedly analyze look of values and the manner that most databases index records isn't always maximum fulfilling for latitudinal queries. Instead, three-dimensional databases practice a spatial catalog to hurry up database processes. Spatial directories are used by spatial record to enhance spatial queries [17]. Directories used by non-spatial records cannot efficaciously cope with landscapes consisting of how a protracted manner factors varies and whether or not and also points collapse inside a longitudinal vicinity of interest.

2. Related Work. Analyzed indexing and question processing in spatial statistics. Indexing strategies are used to boom the velocity of the information retrieval. In the spatio-temporal area the information continuously increases over a time and transferring item dispatched their positions. The principal drawback of spatio-temporal processing is maintaining all of the updates are not possible. The maximum of the indexing methods [1] are best supports few queries and beyond, present and destiny indexing methods shape are very complicated because it is integrated exclusive indexing methods. Interval bushes aren't in particular designed for handling unsure statistics; however one-dimensional uncertain items may be treated as durations by way of using their PDF endpoints. Both indices use a number one tree for layout and secondary structures to save the gadgets at each node, but one has a dynamic number one tree as opposed to a static one. However, the downfall of each interval indices is that if many uncertainty periods overlap with the question intervals endpoints, then few gadgets are pruned from the quest, and a number of times are wasted in calculating chances. The shape of the indexing strategies are very complex so performance is decreased and principal expectation of real time utility is concurrent updation, it isn't supported with the aid of maximum of the indexing strategies. Real time 


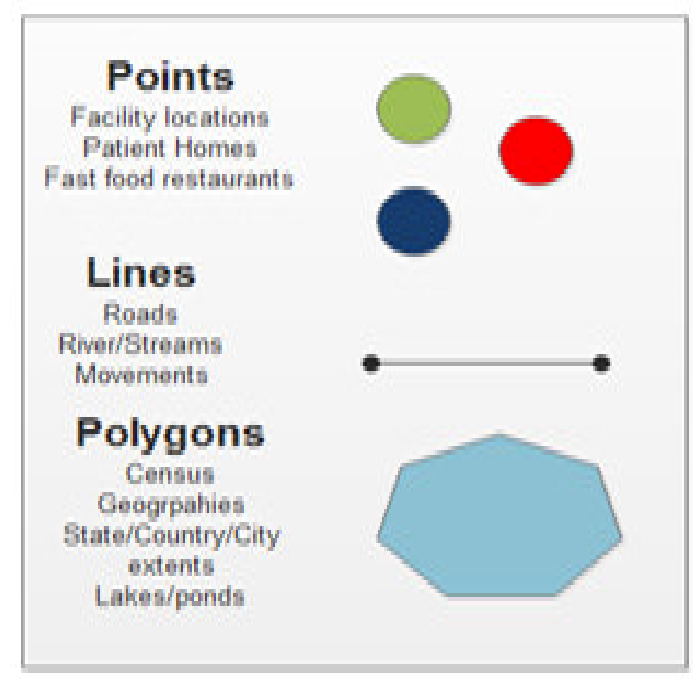

FIG. 1.1. Shapes of Spatial data

application are need comparable object locate and grouping this is also now not possible in exiting indexing techniques and eventually however now not least all of the query processing techniques aren't helps to all the kinds of indexing strategies and queries.

Analyzed spatial statistics control in cloud environments. Principal use an $\mathrm{R}+$-tree [3] to share the facts and the frames inside with go away nodes of the graphic index are preserved as active grids. First, may want to get stability among the grid scopes and then the instances of grid entrees by regulating the 2 limitations, $\mathrm{N}$ besides $\mathrm{n}$, contains of the $\mathrm{R}+$-tree. Additional, in comparison through different variations with the $\mathrm{R}$-tree, the leaf nodes ensure that which is not overlay every feature, and consequently it's distant a assistance as there is no repeated recovery of the equal statistics from special solutions and it remains simple to outline distinctive sources for every rectangle of a leaf child. Moreover, the one task is the manner to layout the vital thing terms of these networks to help effectual inquiries on BigTable manipulates schemes. We positioned the developments of CDMs equally follows: which consumes a quick (key,value) are looking for and it remains to be rapid with Image keys and it can be ordered thru a dictionary format. Based on those traits, we recommend a method to define the critical factor call of a network to help effective queries.

Presented polygon based spatial statistics assessment with map reduce. Geographic Information System which is an expedient intended to seizure, save, work, examine, manipulate, and gift completely styles of topographical facts. Spatial evaluation is a terrific function that is combining with GIS. By way of one vital process in three-dimensional evaluation, polygon overlap, that's a complex geometric set of regulations, combines the spatial and characteristic statistics of dual enter map covers [2]. In such a process, every polygon of a cover is blended with some other layer in couple to reap the cease end result. Classically, particular thematic covers of the identical location are occupied and covered any on pinnacle with opportunity to yield an outcome layer. Increasingly, the dimensions range, and replace charge of a few longitudinal datasets surpasses the ability of three-dimensional computing generation. In a diffusion of times, overlap assessment will become a inefficient venture as commerce with big dimensions of three-dimensional statistics is needed. The computer GIS software normally takes times to perform overlap of this large spatial informations. Such consumption on time is undesirable for lots packages, specifically for actual time coverage choices which encompass predicting which homes would be broken through manner of a transcontinental storm.

Analyzed spatial facts processing using Map-Reduce which is a programming version and computing platform well ideal for parallel computation. In Map Reduce, a application includes a map feature and a reduce function that are consumer-described. The input facts layout is software particular, and is designated by way of the consumer [5]. At first, spatial splitting is adopted to distribute information to all nodes as even as possible, 
and then strip-based two path sweeping set of rules can accelerate the computation instead of spatial index in conventional spatial packages. Finally, pending documents and redundant statistics are used to deal with the relationship between the spatial items. And adopted Map Reduce to process megastar catalog cross certification in astronomical discipline. In order to take full use of the Map Reduce platform, it's far better to make complete issues of parallel algorithm plant. In this section, Map Reduce is carried out to pass-certification, and then in comparison with conventional PostgreSQL DBMS. As a simple and quintessential step, the astronomical cross certification is facing a data avalanche. With the of entirety of latest sky survey tasks and effective telescopes, present day go-certification methods cannot be done on call for large scale astronomical information units. In this paper, Map Reduce framework is delivered to clear up this trouble. The mapping of move-certification algorithm on Map and Reduce phases is carefully taken into consideration. Performance assessment has shown that the Map Reduce-based totally move-certification can outperform the conventional one on PostgreSQL. As our expertise, it is the first attempt to undertake Map Reduce for astronomical go-certification problem.

Implemented partition techniques in spatial hadoop. SpatialHadoop [4] offers a prevalent indexing set of regulations which grow to be recycled to implement network, R-tree, and R+-tree primarily founded for partitioning. This paper spreads preceding observe by way of manner of introducing 4 new partitioning strategies, Hilbert curve, Z-curve, K-d tree and Quad tree and have a look at entirely the seven strategies. The partitioning section of the indexing procedure turns in three steps, in which the initial step is regular and then closing dual steps are custom designed for every partitioning methods. The initial step calculates amount of favorite dividers $\mathrm{n}$ based totally mostly on record length and the HDFS chunk functionality which may be each fixed for complete partitioning techniques. The distribution technique allocates an item to precisely one overlying cell and then the cellular desires to stand increased to surround all controlled statistics. The repetition method eludes growing cells with the useful resource of replicating every data to all overlying cells and query processor consumes to lease a reproduction evading approach to explanation for simulated facts. While range query executed similarly on all of them, we showed that they will be tuned with device parameters which encompass block period in line with the question paintings load. We additionally confirmed the overall performance of spatial be a part of is strongly correlated with the fee of Q1 (average region of partitions) and placed that Quad tree outperformed unique techniques being experimented.

3. Existing Methodologies. The amount of data in spatial databases is developing as greater records are made to be had. Spatial databases in particular store distinct varieties of facts: raster records (satellite/aerial virtual pictures), and vector information (factors, polygons, lines). The complexity and environment of threedimensional databases types them first-class for smearing similar processing [20]. The cutting-edge methods are managing Map-Reduce framework in indexing systems like as $\mathrm{R}$ tree. There are different variation in $\mathrm{R}$-tree that is used of static databases and another one for dynamic databases. The overall performance of R-wood relies upon at the exceptional of the set of rules that groups the data frames on a node.

3.1. Constructing R-Tree with Map Reduce. Let D be a spatial data set composed of devices. Each item i has attributes $j$ i.Id,i.P $\dot{i}$, in which i.Id is referred as the items particular identifier and i.P is also known as objects region in particular three-dimensional domain; different features are possible, but we can supply interest to those quality for R-Tree production cause. R-Tree is consist of minimum bounding rectangles (MBRs) that are created primarily founded on the devices spatial characteristic of i.P. i.Id is used as references to items saved within the RTree in form of leaves. In a main, the spatial objects are divided into nodes. Then, each leaf is processed to generate a minor R-Tree. Finally, the minor R-Trees are combined into the last R-Tree. The principal degrees are carried out in Map Reduce, at the equal time because the remaining segment does not involve large number of computational steps, therefore it is far implemented consecutively out of entries in the cluster [17]. The levels in $\mathrm{R}$ tree index with Map-Reduce are shown in Fig. 3.1. The Major disadvantage with $\mathrm{R}$ tree with Map-reduce is aid only variety queries for future data retrieval in spatial records processing.

3.2. Hillbert R-Tree with Map Reduce. The basic presentation of R-tree relies upon at the great set of rules that groups the frames on a node, at the identical as Hilbert curve [23] which contains the quality of spatial cluster belongings. Hilbert R-tree is extension of Hilbert space-filling-curves approach and in particular the Hilbert value to execute a linear ordering on the information rectangles; then, it negotiates the sorted listing, assigning each set of values at the equal node [20] in the linear ordering, and maximum likely in the 
Phase 1 : Partitioning Function Computation

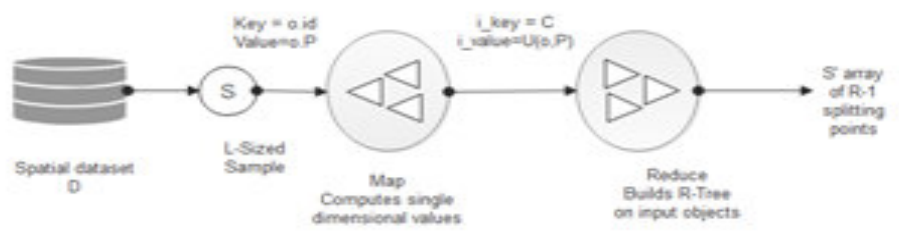

Phase 2: R - Tree construction

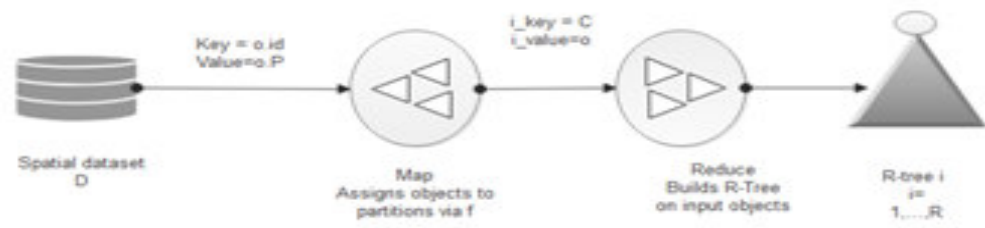

Phase 3: R-Tree consolidation

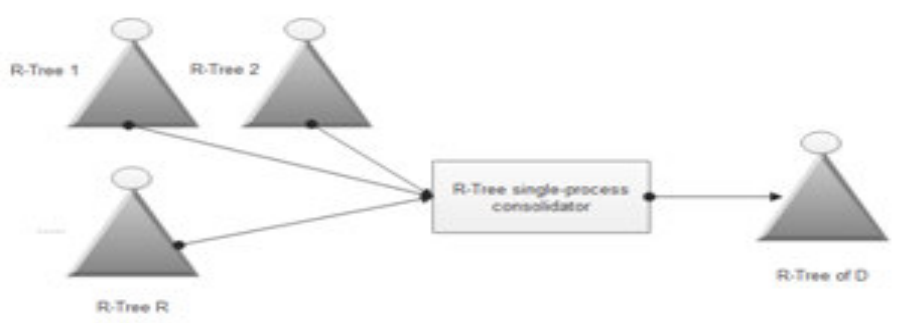

FIG. 3.1. Phases in R-tree indexing in Map-Reduce framework

neighborhood area; therefore, the following R-tree nodes could have the slighter regions. The Hilbert R-tree consists of following structure.

The algorithm of build Hilbert R-tree is given as follows:

- Step 1 Compute the Hilbert cost for each nodes in database.

- Step 2 Categorize statistics data and align as ascending Hilbert values.

- Step 3 Build the R-tree from bottom to up recursively in step with the directive of Hilbert values

The assumption of the algorithm is that the data are static or the frequency of amendment is short. The approach is an easy heuristic for building an R-tree with 100 place of utilization, which at the equal time could have as proper reaction time as viable [18]. In precise, the set of guidelines might be very suitable for parallel bulk-loading processing.

Preliminaries of Hilbert Cost: The Hilbert cost of a node is described due to the fact the Hilbert fee of its center. The manner of calculating the Hilbert value for a data rectangle is split into two steps:

- Step 1 Compute the grid cell that the intermediate of a data are plotted in area

- Step 2 Compute the Hilbert value of the every grid area

Figure 3.2 demonstrates some nodes ordered in a Hilbert R-tree which has the following structure. The Hilbert values of the facilities are the records near the $\mathrm{x}$ symbols (proven simplest for the determine node II). The LHVs are in [brackets].A leaf node contains a most $\mathrm{Cl}$ entries each of the shape ( $\mathrm{R}$, obj id) in which $\mathrm{Cl}$ is the potential of the leaf, $\mathrm{R}$ is the MBR of the real object (xlow, xhigh, ylow, yhigh) and obj-identity is a pointer to the item description document. The vital distinction between the Hilbert R-tree and the $\mathrm{R}^{*}$-tree is that non-leaf nodes also include facts about the LHVs (Largest Hilbert Value). Thus, a non-leaf node in the Hilbert R-tree contains a most Cn entries of the form (R, ptr, LHV) in which Cn is the capacity of a non-leaf node, $\mathrm{R}$ is the MBR that encloses all of the kids of that node, ptr is a pointer to the kid node, and LHV is the 


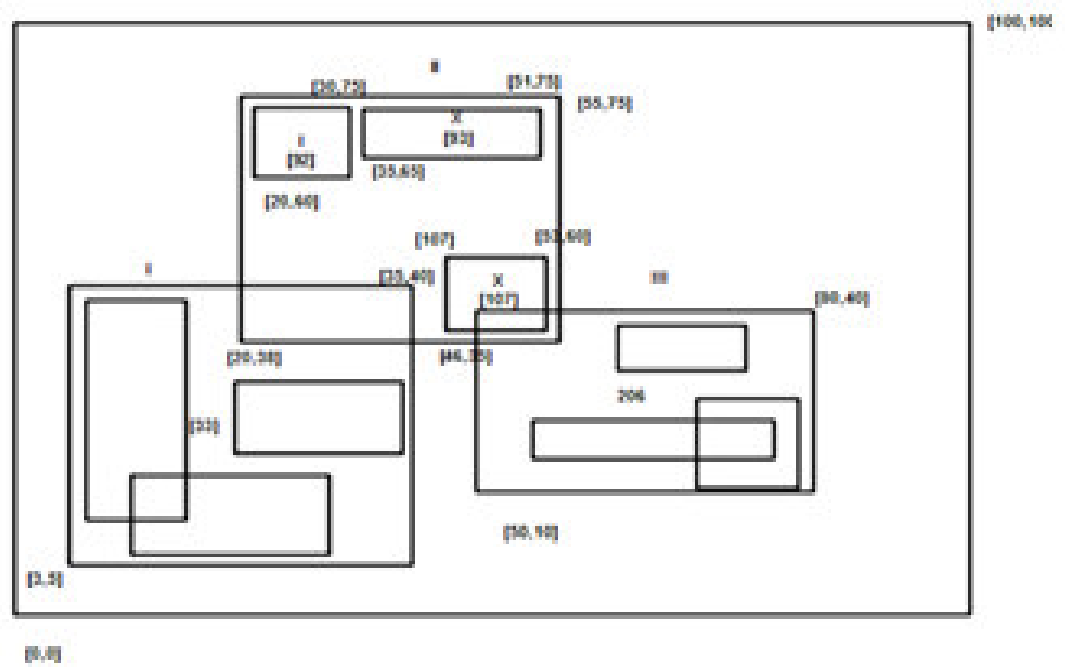

FIG. 3.2. Hilbert $R$ tree structure

largest Hilbert price the various facts rectangles enclosed by means of using R. Notice that because the non-leaf node alternatives one of the Hilbert values of the children to be the price of its non-public LHV, there isn't extra rate for calculating the Hilbert values of the MBR of non-leaf nodes.

4. Rapid Indexing Scheme for Spatial Data Processing in Cloud Enviroment. This work integrates a BR-tree index in MapReduce that results in a parallel B-Tree index. The significance of MapReduceHadoop and indexing in the Hadoop is described below.

4.1. Map Reduce. Large extent of facts has led to adoption of parallel processing. It provides a green processing over a fixed of participating pc machines. It changed into expected that parallel processing might offer new ways of thinking about the prevailing concept of programming language, working machine and storage system for massive dispensed systems. Parallel processing is complex, however many frameworks have evolved that offer parallel processing the use of abstraction to simplify matters. Hadoop, a Java implementation of Map Reduce, has emerged as one such framework. It works on key-price storage concept and has specifically two components, Map Reduce and HDFS. Map Reduce part of the Hadoop encapsulates all info of parallel processing from customers and they get a totally simplified framework for programming. Map Reduce has come to be very famous for parallel dispensation of subjective information. It is mechanism and consists of divide and-conquers technique and pauses a calculation into sub-calculations over established of computer systems in a group that featured as equivalent. Every smaller calculation is dealt with independently and the cease result of the calculation is lower back lower again at a vital issue. A Map Reduce application takes information in the key-cost shape from HDFS and techniques it. It works through well-known features: map and decrease. The map feature accepts input statistics inside the key-price shape and produces a few intermediate facts. Once the map characteristic is completed, reduce feature starts off evolved. The reduce characteristic takes as input the intermediate facts having identical key and produces output facts that is written returned to HDFS. Many map and reduce functions paintings concurrently on one of a kind splits of the enter dataset in HDFS. A huge amount of records switch takes place among the map and decrease features whilst intermediate records are produced. A combiner function can be used to lessen intermediate facts and statistics transfers with the aid of aggregating information on the premise of intermediate key.

4.2. Indexing Scheme With Mpa-Reduce. Initially, MapReduce has been used for large scale records in depth packages for records retrieval from semistructured and unstructured facts. The MR-LSI algorithm retrieves scalable statistics from the unstructured files quite effectively. It has been used correctly in the area of based statistics for expressing queries. Hadoop by default shops facts in key-price form and distribution of 


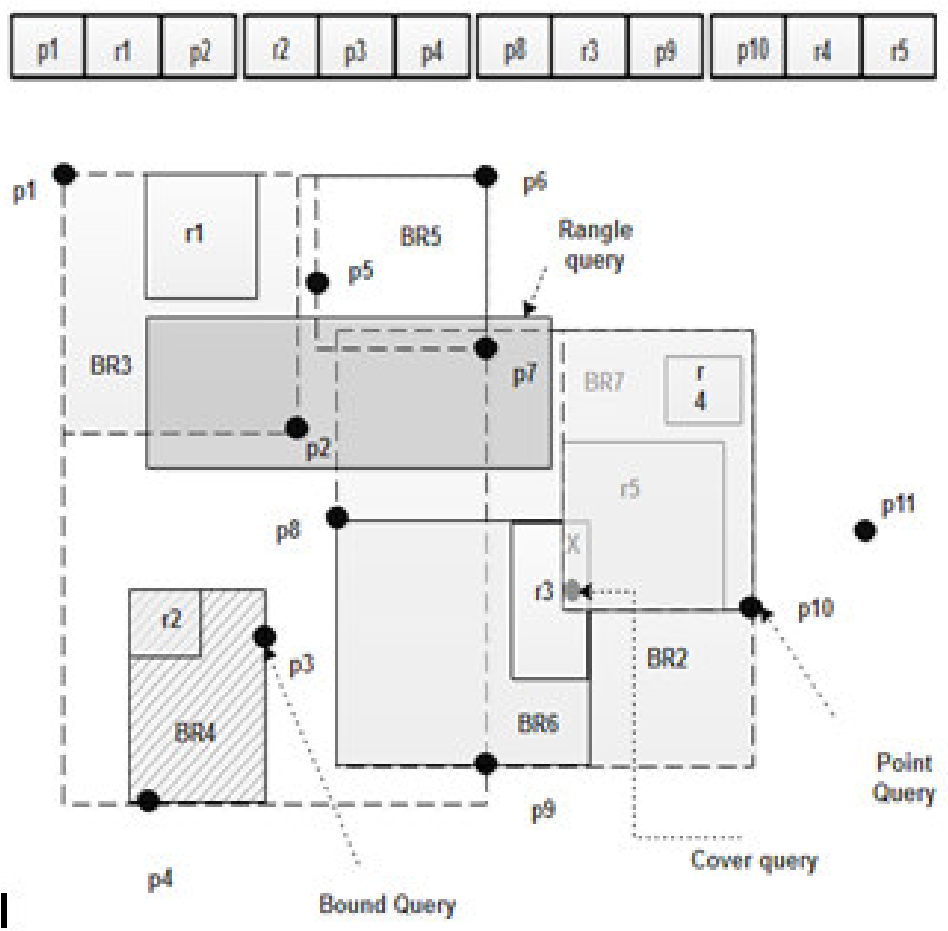

FIG. 4.1. Bloom filter Framework

data over computing nodes takes region via a hash function implemented on keys. The hash feature acts as the primary index for speedy accessing records. However, search over non-key records calls for a secondary index for immediate gaining access to. In the absence of a right secondary index, map tasks are wasted on the undesired dataset. A proper indexing over the input dataset higher organizes the dataset, and minimizes computation. It utilizes map duties for only the desired and filtered dataset, and therefore, improves query resource time and saves machine assets.

4.3. Constructing BR-Tree Indexing in Data Node. Bloom filters base R-tree (BR-tree) in which bloom filter out is integrated to R-tree node. BR-tree is basically R-tree shape for assisting dynamic indexing. In it each node maintains range index to indicate characteristic of gift object. Range query and cowl question supported as it shop object and form of it together. A Bloom clean out is a area-green statistics shape to shop an index of an object and may represent a hard and fast of items as a piece array the usage of numerous impartial hash abilities. BR-tree node is combination of R-tree node and Bloom clean out. BR-tree is likewise loading balanced tree. Overloaded bloom clears out produce excessive duplicate effective probabilities. It reconfigures the multidimensional variety using bounding boxes to cowl item. BR-tree help bound question the first index structure to speak about the certain question. Bound query result into range information of multidimensional function of a queried object. It is not trivial due to the fact BR-tree maintains benefit of Bloom clean out and R-tree each. It mixes the queries like bound question and range question after thing question end result is incredible. BR-tree continues consistency between queried records and the characteristic sure in an integrated shape so that fast point question and accurate sure question viable. The number one format of Bloom clear out is tested in Fig 4.1.

Bloom filters are area-green probabilistic data systems to begin with idea to test whether or not or now not an detail is member of a set or not. Bloom filters have a one hundred keep in mind charge, because faux remarkable suits are viable, whereas fake horrible fits are not possible. It has been validated that Bloom filters are very useful gear within Map Reduce obligations, thinking about that, given the truth that they keep away 
from fake negatives, they allow for doing away with beside the point facts at some point of map levels of Map Reduce duties, as a result configuring themselves as a very dependable area-green answer for Map Reduce. The gain of Bloom clean out is space overall performance. The length of the Bloom clear out is constant nevertheless of the variety of the features $\mathrm{n}$, however there may be a tradeoff among $\mathrm{m}$ and the false high pleasant opportunity p. The opportunity of a fake high great after placing $n$ factors can be intended as follows:

$$
p=\left(1-(1-1 / n)^{k m}\right)^{k} \approx\left(1-e^{(k m / n)}\right)^{k}-\operatorname{Eqn}(1)
$$

\subsection{Execution of proposed work.}

1. Job suggestion. If an activity is acquiesced, $\mathrm{m} 1$ is the map task for $\mathrm{R}, \mathrm{m} 2$ is map task for $\mathrm{S}$, and $\mathrm{r}$ reduce duties are created. A task contains all crucial data to be track on a task tracker which incorporates the attention formation and the area of the matching input/output records.

2. First map phase. Activity tracker allocates the m1 map obligations or the reduce duties to lazy assignment trackers. The map task tracker reads the entire data and split it for the assignment, converts it to key or value pairs, and then performs the map feature for the every input dataset.

3. Local filter construction. The in-between pairs comprised of the map function are separated into $r$ spilts, which may be dispatched to $\mathrm{r}$ project trackers respectively. Moreover, Bloom filters are built at the bases in every partition and referred as filters in the name of community filters due to the fact they may be constructed for simplest the in-between effects in a sole undertaking tracker. If a venture tracker runs more than one map duties, it merges the close by filters of every assignment and then continues merely $\mathrm{r}$ filters.

4. Global filter merging. After all m1 map obligations are whole, the process tracker indicators all challenge trackers to ship the nearby filters through heartbeat messages. Then, all assignment trackers send their community filters to the process tracker, and then process tracker concepts the overall filters to the dataset R. Next, the process tracker sends the overall filters to all project trackers. Until constructing and transmitting the global filters that are whole, the map tasks to the dataset $\mathrm{S}$ aren't allocated.

5. Additional map segment. Then job tracker allocates the $\mathrm{m} 2$ map duties or the enduring lessens duties with task trackers. Task trackers track the allotted project with the established international filters. The enter key or cost pairs cant be set inside the global filters for filtered out.

6. Reduce segment. This step is the same as the lessen section in Hadoop. A reduce task tracker reads the corresponding intermediate pairs from all map task trackers the usage of far flung system calls. It kinds the all intermediary pairs and runs the lessen characteristic. Final output outcomes are written inside the given output course. We have made modifications to the layout of Hadoop. First, we includes map task inside the instruction of the information. Second, include assemble Bloom filters at the build enter in allotted fashion to clear out the probe enter. The proposed format is shown in Fig.4.2.

The procedure of MR-BR-Tree takes inputs as various data types and produce output as spatial index. The pseudo code of procedure is described as follows:

\section{Algorithm 1: Rapid Indexing for Spatial Data (RISD)}

Input: Spatial Data points or Lines or Polygons $\mathrm{D}=\left\{d_{1}, d_{2}, \ldots, d_{n}\right\}$

Output: Index tree $I_{E}$

Step 1: for all data types (d) in D do

Step 2: spilt data into partitions $P_{i}$

Step 3: Normalize $d_{i}$

Step 4: Compute (key,value) c of $P_{i}$ using BR tree and add C

Step 5: end for

Step 6: I=BuildBRtree() as $\operatorname{Eqn}(2)$

Step 7: Send Index $I_{E}$ to admin

In this partition algorithm, we can read the spatial datasets as input they can be as many types. 


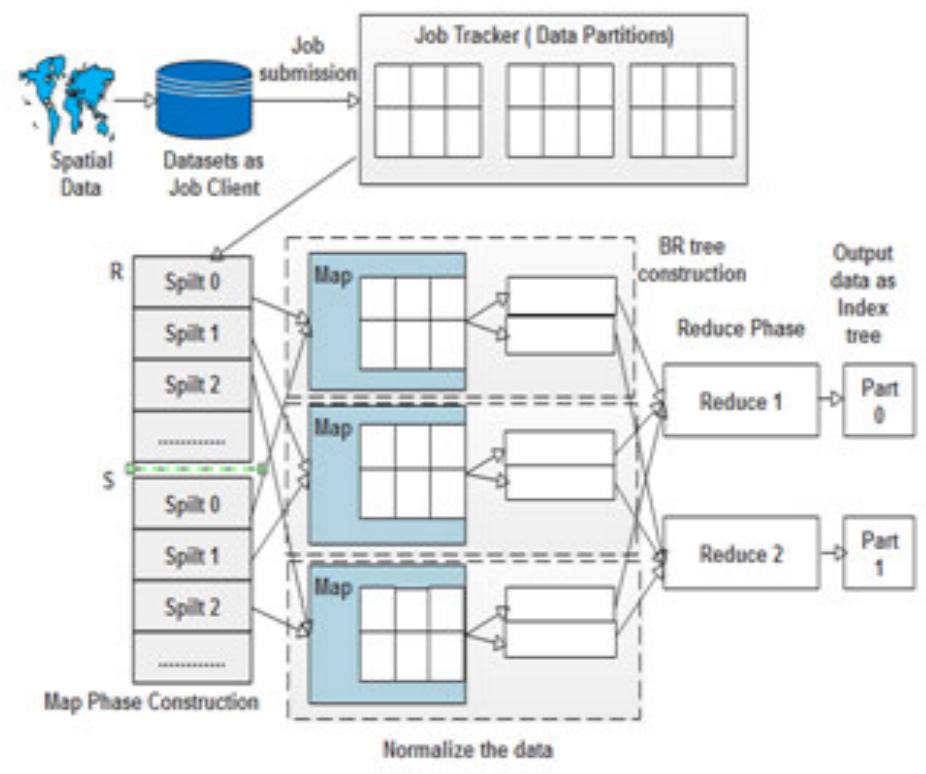

Fig. 4.2. Proposed Framework

Input: Spatial points D

\section{Algorithm 2: Partitioning algorithm}

for all object Obj in mapper phase do

if $(D[i-1]<O b j$. Value $\leq D[i])$

Send Obj to reducer i

end for

This guarantees that the result of reducer $i$ is less than the result of reducer $i+1$. Formerly, every reducer categories each map records directly and writes them into single partition task, the ones looked after partition documents shape a globally taken care of document. Each spatial statistics factor di in D is normalized to [0, 1$]$.

4.5. Build Bloom filter R Tree:. While a task tracker runs a map undertaking of the build the data and send this data as input, and it makes the Bloom filters as intermediate records made out of the challenge. A Bloom filter is shaped for every map output partition allocated for each reduce assignment, and therefore the general wide variety of the Bloom filters is the amount of lessen obligations. When more than one map duties are run on a undertaking tracker, the challenge tracker merges each Bloom filters from the responsibilities to keeps simplest unique kind of Bloom filters. And call this traditional of Bloom filters as close by filters. When complete map tasks aimed at the construct input are comprehensive, the pastime tracker has to fold all neighborhood filters to collect the global filters. And upload Task Tracker Action lessons, known as Local Filter approach and Receive Global Filter Action, for the Global filter approach.

The task tracker shows the Local filter action is in the form of heartbeat reaction to entire challenge trackers, and that they direct the task tracker their native filters. The activity tracker joins all of the nearby strainers to construct the worldwide sifters the usage of bitwise OR processes, and mentions the action as Receive Global Filter Action by means of the global filters in the heartbeat message towards total task trackers. The conversation price aimed at the filter for analyzed $\mathrm{Cf}$ is

$$
C f=2 c t \cdot n \cdot r e \cdot t k-\operatorname{Eqn}(2)
$$

wherein ct is the amount to allocation data from single node to another, $\mathrm{n}$ remains the dimensions of Bloom filter, re is the wide variety of lessen tasks, and tk is the amount of challenge trackers. The coefficient 2 is accelerated for the reason that neighborhood filters and with the global filters are transmitted among the task tracker and the project trackers. Our proposed structure significantly improves the execution time of queries. 


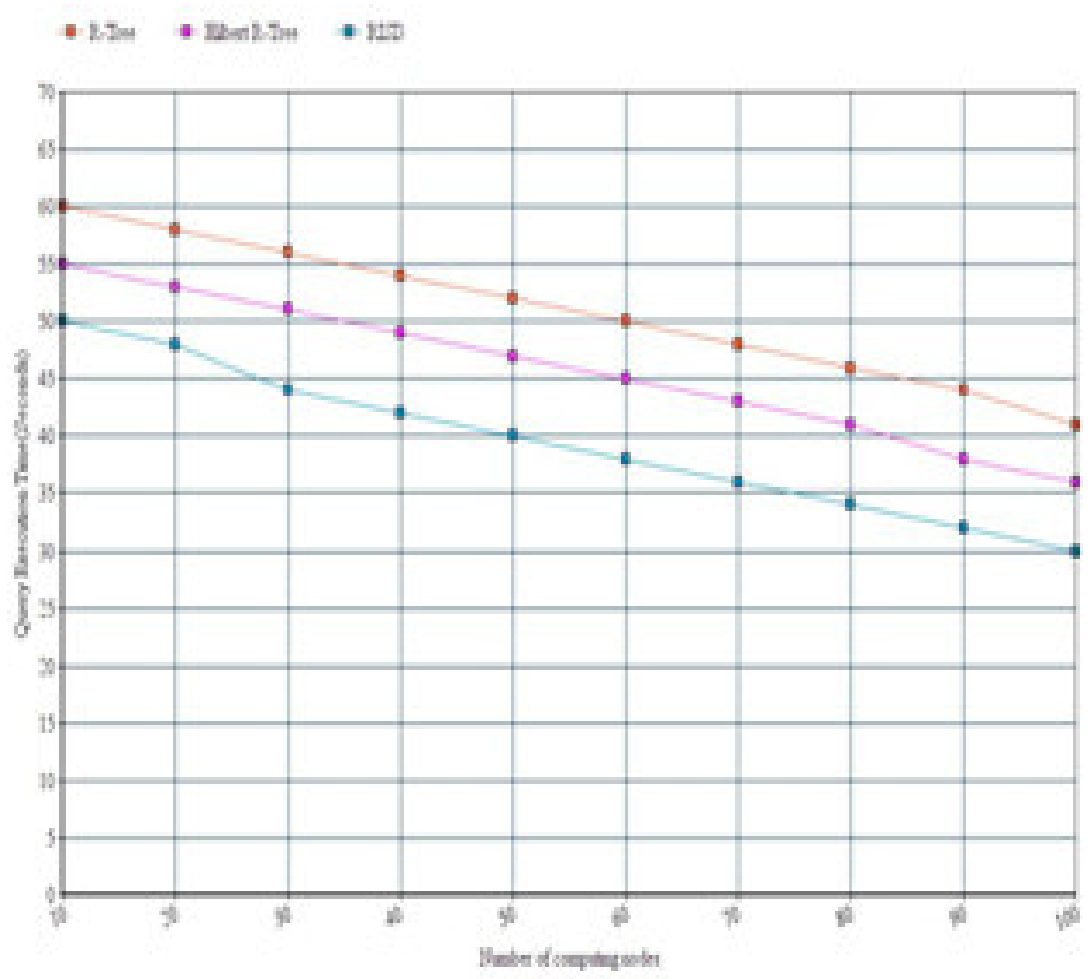

5. Experimental Results. Experiments are carried out for performance evaluation of search query on MR-BR-Tree indexed MapReduce The search query execution time of the R, Hilbert R and RISD Index on spatial data. The time taken to distribute the partitioned data onto cluster nodes and managing a global index of the master node is almost similar in all the three approaches. The query execution time shrinkages as the size of cluster upsurges. It remains due to parallelization of query. The query completing time is composed of the amount of time elapsed in map and reduce phases. The search query is decomposed into sub-queries that run in parallel, and consequently, execution time decreases. Initially, there is a small rise in execution time when second and third node is added to the cluster. It is due to increased sorting and shuffling in intermediate stages that overcomes the gain in performance due to fast computation achieved with parallelization. But later on, the performance gain achieved with parallel computation exceeds the burden of sorting and shuffling. Consequently, execution time decreases gradually with the addition of nodes in the cluster. In our proposed RISD framework, the index is built only one time on the complete dataset and only range search queries execute on the index. The indexing of the data reduces query execution time. The Performance chart is shown in Fig 5.1. The RISD performs almost better than the B-Tree and Hilbert $\mathrm{R}$ tree in spatial datasets.

6. Conclusion. In this work we studied and implemented the performance of bloom filter R-Tree in cloud platform. The results show that when using an instance with greater resources a better performance will be gained. The Map-Reduce technology has proved very effective for large scale structured, semi-structured and unstructured data, for information processing and retrieval. In this connection, a B-Tree index, in a chainedMapReduce process, is designed and implemented. The proposed work compares various indexed data structures that include RISD, R, Hibert R of Hadoop, for range search queries. It is observed that a significant amount of time is less to build indexes in proposed system than the existing system. 


\section{REFERENCES}

[1] John, A., M, Sugumaran and R. S. Rajesh, Indexing and Query Pprocessing Techniques in Spatio-Temporal Data, ICTACT Journal on Soft Computing 6.3, 2016.

[2] Wang, Kai, Jizhong Han, Bibo Tu, Jiao Dai, Wei Zhou, and Xuan Song, Accelerating spatial data processing with mapreduce, Parallel and Distributed Systems (ICPADS), 2010 IEEE 16th International Conference on. IEEE, 2010.

[3] Eldawy, Ahmed, Louai Alarabi, and Mohamed F. Mokbel, Spatial partitioning techniques in SpatialHadoop, Proceedings of the VLDB Endowment $8.12,1602-1605,2015$.

[4] Wang, Yong, Zhenling Liu, Hongyan LiaO, And Chenguun Li, Improving the performance of GIS polygon overlay computation with MapReduce for spatial big data processing., Cluster Computing 18.2, 507-516, 2015.

[5] Wei, Ling-Yin, Ya-Ting Hsu, Wen-Chin Peng, and Wang-Chien Lee, LU-Indexing spatial data in cloud data managements, Pervasive and Mobile Computing 15 (2014): 48-616.

[6] Rajashekhar M. Arasanal and Dannish U. Rumani, Improving MapReduce Performance through Complexity and Performance Based Data Placement in Heterogeneous Hadoop Clusters, Distributed Computing and Internet Technology, Lecture Notes in Computer Science Volume 7753, 2013, pp. 115-125, 2013.

[7] Performance Measurement of a Hadoop Cluster, , http://www.acma.com/acma/pdfs/AMAX Emulex Hadoop Whitepaper.pdf (Accessed on December 20, 2015

[8] Jeffrey Dean and Sanjay Ghemanat, MapReduce: SimplifiedDataProcessing on Large Clusters, Magazine Communications of the ACM - 50th anniversary issue: 1958 2008, Vol. 51 Iss. 1, pp. 107-113, 2008.

[9] http://sortbenchmark.org/Yahoo2009.pdf (April 2009) (Accessed on February 22, 2016).

[10] M.K. Agullera, W. Golab, and M.A. Shah, A practical scalable distributed b-tree, PVLDB, Vol. 1, Iss. 1, pp. 598-609, 2008.

[11] Burhan Ui. Islam Khan, Rashidah F. Olanrewaju, Hunain Altaf and Asadullah Shah, Critical insight for MapReduce optimization in Hadoop, International Journal of Computer Science and Control Engineering, Vol. 2, Iss. 1, pp. 1-7, 2014.

[12] Matei Zaharia, Andy Konwinski, Anthony D. Joseph Randy Katz and Ion Stoica, Improving MapReduce Performance in Heterogeneous Environments, In Proceedings of the 8th USENIX conference on Operating systems design and implementation, pp. 29-42, USENIX Association Berkeley, CA, USA 2008.

[13] Jimmy Lin And AleK Kolcz, Large-scale machine learning at Twitter, Proceedings of the 2012 ACM SIGMOD International Conference on Management of Data, Scottsdale, AZ, USA, pp. 793 804, 2012

[14] Fan Zhang, Junwei Cao, Samee U. Khan, Keqin. Li and Kai Hwang, A task-level adaptive MapReduce framework for real-time streaming data in healthcare applications, Future Generation Computer System, Vol. 43 44, pp. 149 160, 2015.

[15] David A. Patterson, Technical perspective: the data center is the computer, Communications of the ACM, Vol. 51, Iss.1, pp. 105-105, 2008.

[16] Eric Anderson and Joseph Tucek, Efficiency matters!., ACM SIGPOS Operating Systems Review, Vol. 44, Iss. 1, pp. 40-45, 2010.

[17] Feng Li, Beng Chin Ooi, M. Tamer Ozsu and Sai Wu, Distributed Data Management Using MapReduce, ACM Computing Surveys, Vol. 46, Iss. 3, Article No. 31, 2014.

[18] H.V. Jagdish, B.C. OoI, AND Q.H. Vu. BATON, A balanced tree structure for peer-to-peer networks, In Proceedings of the 31st International Conference on Very large data bases, VLDB Endowment, pp. 661-672, 2005.

[19] F.N. Aftari And J.D Ullman, Optimizing joins in a MapReduce environment, In Proceedings of the 13th International Conference on Extending Database Technology, EDBT, pp. 99-110, 2010.

[20] Sai Wu, Dawei Jiang, Beng Chin Ooi, and Kun-Lung Wu, Efficient b-tree based indexing for cloud data processing, Proceedings of VLDB Endowment,Vol. 3, Iss. 1, pp. 1207-1218, 2010.

[21] Ian H. Witten, Alistair Moffat, and Timothy C, Bell. Managing Gigabytes: Compressing and Indexing Documents and Images, Morgan Kaufmann, ISBN 1558605703, 1999.

[22] Steffen Heinz and Justin Zobel, Efficient single-pass index construction for text databases, JASIST, Vol. 54, Iss. 8, pp. 713-729, 2003.

[23] Anthony Tomasic and Hector Garcia-Molina, Performance of inverted indices in shared-nothing distributed text document information retrieval systems, Proceedings of PDIS, pp. 8-17, 1993.

[24] Mike Cafarella and Doug Cutting, Building Nutch: Open source search, ACM Queue, Vol. 2, Iss. 2, pp. 54-61, 2004.

[25] Mark Lillibridge, Kave Eshghi, Deepavali Bhagwat, Vinay Deolalikar, Greg Trezise and Peter Camble, Sparse indexing: large scale, inline deduplication using sampling and locality, In proceedings of USENIX Conference - File and Storage Technologies (FAST), pp. 111-123, February 2009.

[26] Yang, Chaowei, Michael Goodchild, Qunying Huang, Doug Nebert, Robert Raskin, Yan Xu, Myra Bambacus, and DANIEL FAY, Spatial cloud computing: how can the geospatial sciences use and help shape cloud computing?, International Journal of Digital Earth. 4(4) pp. 305-329, 2011.

Edited by: Rajkumar Rajasekaran

Received: Jul 24, 2018

Accepted: Dec 14, 2018 



\title{
ENHANCED DATA SECURITY FOR PUBLIC CLOUD ENVIRONMENT WITH SECURED HYBRID ENCRYPTION AUTHENTICATION MECHANISMS
}

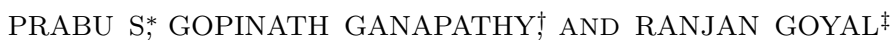

\begin{abstract}
Cloud computing is an evolving computing technology that provides many services such as software and storage. With the introduction of cloud storage, the security of outsourced data has become a major issue in cloud computing. Data storage in cloud computing environment needs to be secured in order to provide a safe and foolproof security for data outsourcing of the cloud service users. This paper presents a model for security of data in public cloud storage environment which successfully detects the unauthenticated access or any anomaly in the data. The proposed authentication model along with the data security model presented in this paper shows that this model is the best model suitable for securing the data in cloud computing environment.
\end{abstract}

Key words: Cloud Computing; Data Outsourcing; Data Security; Encryption model; Data Storage.

AMS subject classifications. 68M14, 68P25, 68P20

1. Inntroduction. Cloud Computing is an internet based computing technology that provides many services to the users including cloud storage. The cloud storage is a kind of cloud computing service that provides storage of data in logical pools, that is, the data is actually stored in some physical data centers present in some other location, but the user can access the data anywhere and on any device. The cloud computing is actually not a new technology, rather it is evolved from many existing technologies including grid computing, utility computing, parallel computing, distributed computing and virtualization. The cloud computing is a very powerful environment having pool of thousands of connected servers [1].

The cloud computing environment can be deployed as public, private, community or hybrid model. The public cloud deployment model is openly accessible to the public. The use of public deployment model can help in reducing computing costs making it economically inexpensive and efficient. The private cloud deployment model is generally used by private organizations. This model utilizes the VPN (Virtual Private Network) which makes it more secure from outside intrusion as compared to public cloud model. The community cloud deployment model is shared by several organizations that leads to formation of a community. This model is only suitable for the organizations working on the similar projects. The hybrid model can be a combination of public, private or community models. Here, it is a notable point that among all the deployment models, the public cloud model is most insecure and generic model. The public model can be used by anyone irrespective of the fact whether the use is a part of organization or community thus making it a generic cloud model. Thus, data security to the public cloud model is required to be provided in order to secure the outsourced data of the users.

This paper presents a model for enhanced data security for public cloud environment. The paper provides the description of model and its analysis which shows that the proposed model is suitable for enhanced security of data in public cloud environment. Rest of the paper is organized as follows: Section 2 provides the description of the cloud security with respect to attacks and security issues, fraud detection and different security mechanisms. Section 3 provides a discussion on related works based on the data security issues and reviews of cloud storage environment. Section 4 provides detailed discussion on the existing security mechanisms used for proposing the hybrid model. Section 5 provides the proposed model for enhanced security in (public) cloud environment. Section 6 provides an analysis on the proposed model followed by the conclusion in Section 7 .

2. Cloud Security. Cloud Computing though being a powerful architecture, is vulnerable to many security issues and attacks. A discussion on the attacks, fraud detection and security mechanisms are provided below.

2.1. Attacks and Security Issues. There are several number of possible attacks to which the cloud storage is vulnerable. The attack can be a physical or a network based. The physical attack can be the attack from an unauthorized person, trying to access the data in cloud storage by breaking into the authentication

\footnotetext{
*School of Computer Science, Engineering and Applications, Bharathidasan University, Tiruchirappalli, India

${ }^{\dagger}$ School of Computer Science, Engineering and Applications, Bharathidasan University, Tiruchirappalli, India

${ }^{\ddagger}$ School of Computing Science and Engineering, Vellore Institute of Technology, Vellore, India
} 
mechanisms. The network based attacks are the attacks from a system or a network of systems that are controlled by an attacker in order to break into the storage system or to disrupt the service. The most common among the network based attacks are Distributed Denial of Service (DDoS), Man-in-the-Middle (MITM) attack. In DDoS attack, the attacker sends too many meaningless packets or requests which the system fails to handle and the service gets disrupted [2]. In case of MITM attack, the attacker sits between two parties and tries to acquire the information or data being exchanged [3]. This creates a major security issue for data outsourcing.

2.2. Fraud Detection. The detection of any fraud or attack is an important factor deciding the level of security in the system. The data outsourced should be kept into an environment equipped with a fraud detection mechanism. The detection can help in taking further actions to prevent any theft or loss of data. The mechanism of fraud detection may have several factors such as usual locations of login and possible places the user can login in a particular time duration. For example, one user logging in from New York cannot login just after 10 minutes from Hong Kong. Nonetheless, there can be a verification process initiated in that case. Further, the usual activities of a user activities can help in the prediction of fraud [4]. The work does not consider the fraud detection in terms of the example given above as the model proposed in the paper eliminated such scenario from getting executed.

2.3. Security Mechanisms. There are many possible mechanisms for security of data in public cloud. The cryptography algorithms can be used for encryption of the data and sensitive information. The cryptography is a technique which can be used to securely transfer the information between two parties. The data can be encrypted with the use of algorithms, so the attacker cannot read the actual data even in case the attacker acquires the data. There can be a private and public key which can be used to decrypt the data on successful transmission [5].

3. Related Works. Sugumar et al. [6] provided a detailed demonstration about the security issues, characteristics and its importance in public cloud storage environment. The two models proposed were based on the owners and users of the service. In case of any sensitive data stored, the owner and the users are treated as different, otherwise as same. The security issues and requirements addressed in the paper suggested that there is a need of new mechanism for ensuring of outsourced data. Singla et al. [7] explored the security of data and data at moving. The paper proposed an authentication protocol mechanism that was based on a 3-step process and a block cipher based encryption algorithm was proposed in the paper to prevent against known attacks. The first step in the 3-step in the authentication protocol mechanism is sending the message to the client on demand. The second step involves the client responding to the message with a value that is calculated using a one-way hash function. The third step involves authentication by verification of the response value against its own calculated hash value. In case of a match, the cloud service will be offered by to the client, otherwise the connection will be terminated

Masala et al. [8] presented a cloud platform that was designed to provide secure access to the data stored in the cloud. The cloud platform was built using open stack architecture and the authentication was done using biometric fingerprint and face recognition. Kaaniche et al. [9] provided a review of data security and privacy preservation in cloud storage environment using cryptographic mechanisms. The comparative analysis of different cryptography based defense mechanisms and the work done in the paper shows that the there are high security and privacy challenges that are required to be solved with evolving cloud infrastructure. A survey of security issues for cloud computing presented by Khan [10], analyzed and categorized the working mechanisms of possible security issues and its surveyed some of its possible solutions. The paper also provided a survey on intrusion detection and prevention systems and analyzed the effectiveness of the system. The literature suggested countermeasures to deal with the security issues discussed in the paper.

The literature survey of the related works showed that the public cloud environment is vulnerable to many network-based attacks and there is high need to solve this issue by providing a foolproof authentication protocol and encryption mechanism for proper authentication and authorization of users. Thus, this paper provides a hybrid authentication mechanism which on analysis shows that the mechanism is highly secured from severe network-based attacks. Further, the model proposed in this paper, secures the data in rest and data in transit by making it highly tough for the attacker to enter into the environment and remain into the environment. 


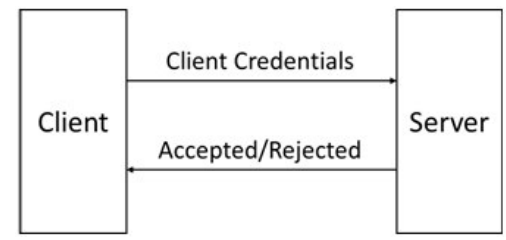

FIG. 4.1. PAP 2-way handshake mechanism

4. Existing Mechanisms. The existing mechanisms for authentication and encryption are discussed as follows:

4.1. Authentication Protocols. There are several existing authentication protocols proposed by researchers and scientists. The most common among them used to build the proposed mechanism includes Password Authentication Protocol (PAP), Secure Socket Layer (SSL) and Challenge Handshake Authentication Protocol (CHAP). This paper only focuses on these methods to provide a multi-layered hybrid authentication protocol.

The PAP is a password based authentication protocol that provides two-way handshake mechanism. In this protocol, the client sends the details such as username and password to the server which gets verified on the server side. In case the credentials match with the data present on the server-side database, the server sends an acceptance acknowledgement to the client and a connection is established. In case of no match, the client request is rejected and the connection request is terminated. Fig. 4.1 visualizes the PAP mechanism.

The issue in this protocol is that this protocol is highly vulnerable to network attacks such as Man-In-TheMiddle Attack (MITM) which can lead to account hijacking and unauthorized access. This issue occurs in the case of PAP as the credentials sent by the client are in clear or plain text, i.e. not encrypted. This issue is fixed up to some extent by encrypting the credentials with some cryptographic algorithm. Nonetheless, there is still a chance that the attacker can decrypt the credentials making it still vulnerable to the MITM attacks.

The Secure Socket Layer (SSL) is a protocol used to establish a secure connection between the server and the browser of the client by the use of encrypted links to make the transactions private. The SSL uses a public and private key. The public key is used for asymmetric encryption and private key is used for symmetric encryption. The use of asymmetric key provides better authentication and the use of symmetric key provides faster authentication. A SSL certificate is required to establish a SSL connection for which the submission of Certificate Signing Request (CSR) is required to be done to the Certification Authority (CA). The CSR contains the details and identity of the website along with the private key. The CA then validates the data and issues a SSL certificate which matches with the private key. Thus, the SSL is based on mutual authentication i.e. the digital certificates verify the web servers and client identity before the establishment of the connection. If the web page is SSL secured, then the web address will begin with HTTPS instead of HTTP and a lock icon with appear which contains the details of the website certificate. Also, if the SSL is having Extended Validation (EV) certification then a green address bar will appear instead of the usual address bar. The use of SSL can prevent fraud and hijacking as it relies on encryption and the originality of the website is also verified up to some extent but it may cause a slowdown in the performance. Thus, the SSL can be used along with the proposed model but the model did not consider the use of SSL. Another protocol is Extensible Authentication Protocol (EAP). EAP is an authentication framework. The EAS was originally an extension for Point-to-Point (PPP) authentication. EAP framework supports multiple authentication mechanisms. This framework can also be used but the proposed model did not consider the complete use of this framework.

Another method of authentication is Challenge Handshake Authentication Protocol (CHAP). It is an internet standard that uses one-way MD5 hash function. The CHAP is based on a 3-way handshake mechanism [11]. In this type of authentication, instead of the actual password, the transmission of the hash result is performed over the network. As the password in this case, does not get transferred over the network, it cannot be captured during the transmission. Also, the hash function generates the random string in such a manner that the operation cannot be reverse engineered to obtain the original password. Nonetheless, this method is vulnerable to remote server impersonation. This vulnerability can be prevented by using a two-way authentication 


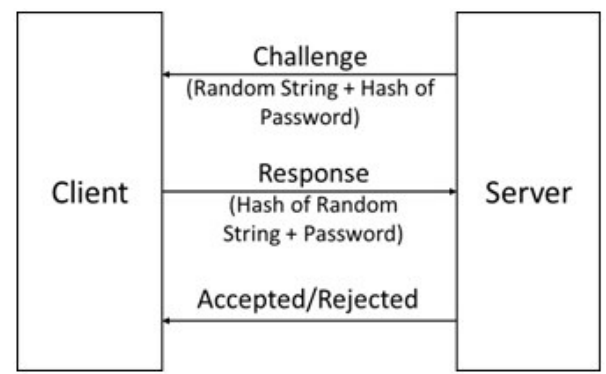

FIG. 4.2. CHAP 3-way handshake mechanism

using separate keys for transmitted and received data to identity server as well as client. This concept is used in MS-CHAP in which the challenge is sent repeatedly during the connection. Fig. 4.2 visualizes the CHAP mechanism.

4.2. Data Security. There are several existing algorithms proposed by researchers and scientists. The most suitable is discussed in this paper that is used in proposing the encryption model, i.e. Advanced Encryption Standard (AES). The AES is an encryption standard that is actually the 128-bit based symmetric block cipher algorithm known as Rijndael algorithm [13-15]. The AES is being used worldwide and is the most secure standard at present. The AES is the successor of Data Encryption Standard (DES) [16]. The AES is faster and more secure than DES. The Rijndael algorithm was based on 128, 192 and 256-bits block size but for the AES, only 128-bit block size was accepted. The AES thus have the block size of 128-bits and key size of 128, 192 and 256-bits. The algorithm is based on combination of substitutions and permutations. It operates on a 4 by 4 column major order matrix. There are 10 rounds for 128-bits key, 12 rounds for 192-bits key and 14 rounds for 256-bits keys. Thus, the data-in-rest and data-in-transit can be handled securely using this algorithm. The data here refers to the plain text which on encryption becomes cipher text. The cipher text can be converted back to plain text with the help of decryption. In symmetric encryption, the same key is used for encryption and decryption. Fig. 3 depicts the AES encryption and decryption operations.
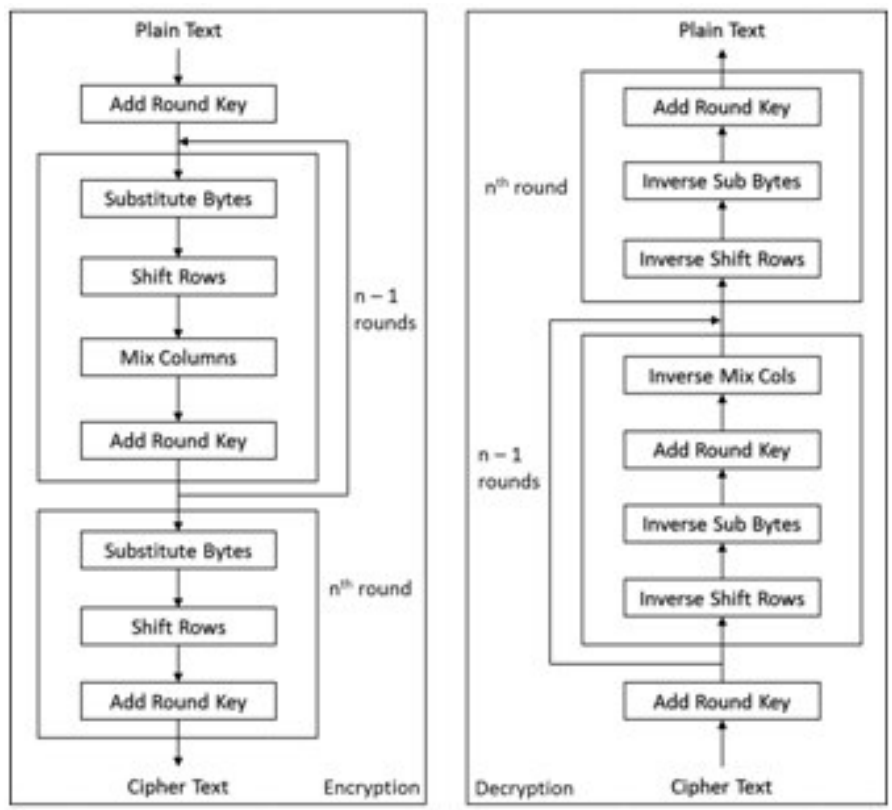

FIG. 4.3. AES Encryption and Decryption 


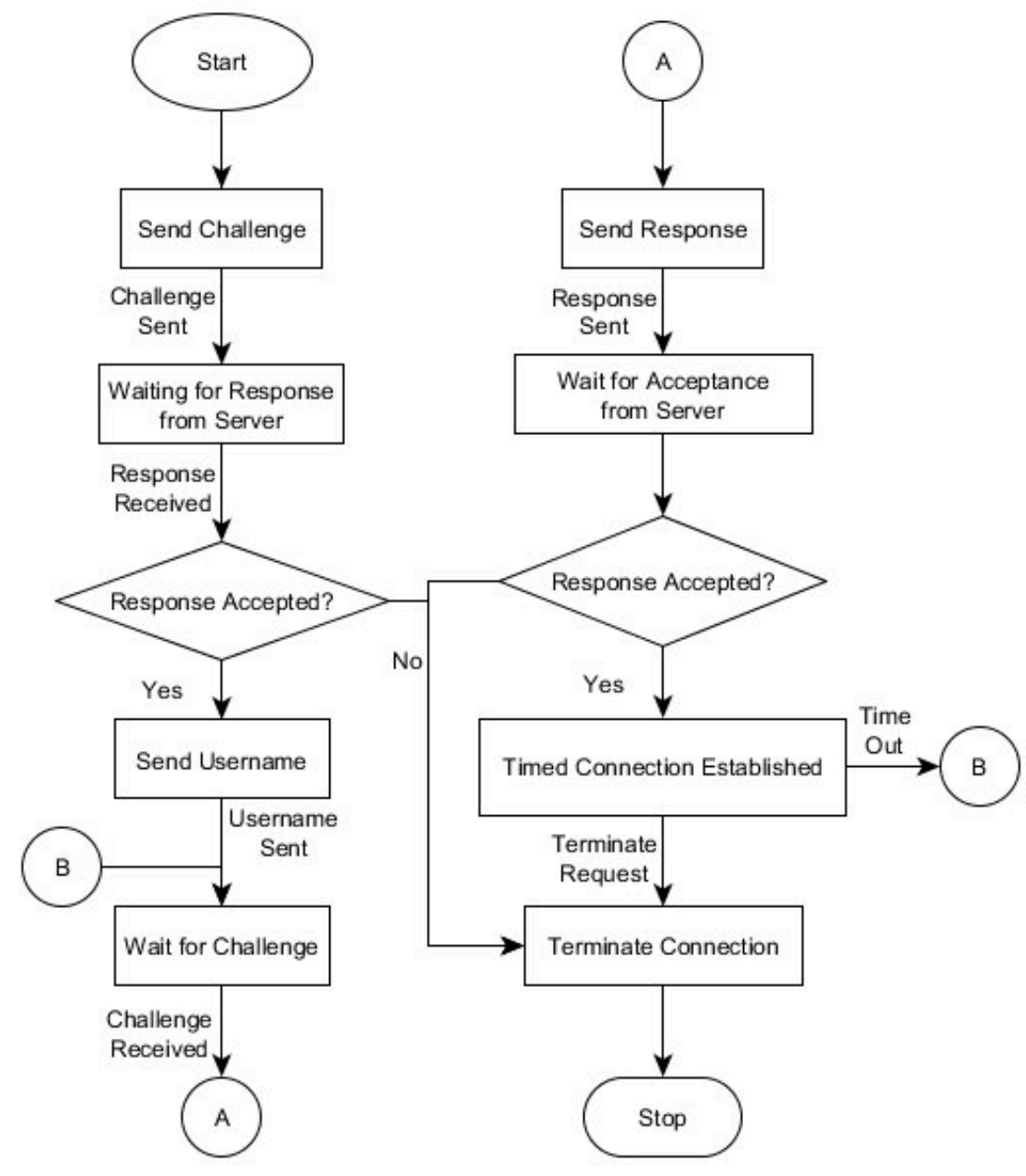

FIG. 5.1. Proposed Client-Side Authentication Mechanism

5. Proposed Method. The paper focuses on securing data present in the public cloud computing environment by providing a more secure hybrid of authentication and encryption mechanisms. The two mechanisms are discussed as follows:

5.1. Authentication Mechanism. The authentication protocol proposed in this paper, works on the idea of the PAP and CHAP mechanisms along with TLS. The client-side mechanism for the proposed authentication protocol is depicted in the Fig. 5.1. The client sends a challenge to the server and waits for the response. After the response is received, the response is verified and is either accepted or rejected. If the response is accepted then the client sends the username in encrypted format and waits for the challenge. In case of rejection, the connection is terminated. When the challenge is received, the response is generated and is sent to the server and the client waits for the acceptance of response from the server. Upon Acceptance, a timed connection is established. In case of rejection, the connection request is terminated. Upon connection time-out, the client waits for the challenge from the server and same procedure is repeated until the connection gets established again. During the connection, any terminate request if given, terminates the connection.

The server-side mechanism depicted in Fig. 5.2, shows the authentication procedure from the server side. Initially, the server waits for the challenge from the client. When the challenge is received, a response a generated and sent to the client. If the response is accepted by the client, the server waits for the client to send 


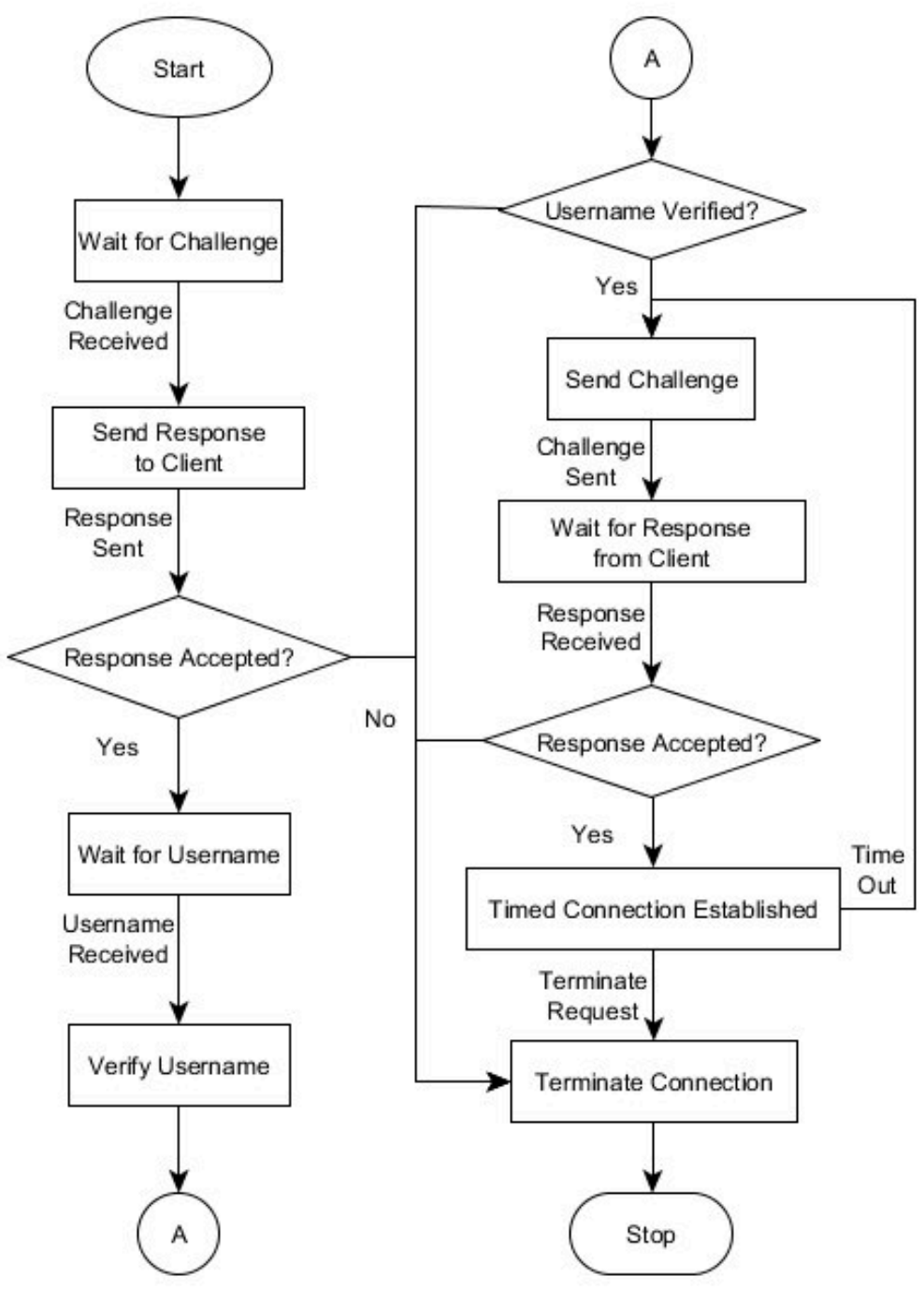

Fig. 5.2. Proposed Server-Side Authentication Mechanism

the username. When the client sends the username, the server verifies the username by decrypting encrypted using and matching it in the database. If the username is successfully verified, the server sends a challenge to the client and waits for the response. When the response is received, it is verified and on acceptance of response, a timed connection is established. The mechanism leads to termination in the case of rejection of response, rejection of username or any termination request.

The combined Client-Server Authentication Mechanism is depicted in Fig. 5.3. The mechanism depicted in the figure is based on the interactions of the client and server that does not show the time of propagation and delay. This proposed mechanism is named as Improved Hybrid Authentication Mechanism (IHAP).

5.2. Data Security. The paper proposes the encryption mechanism using the AES algorithm. There are several attacks that AES can handle including brute force and biclique attack due to high time complexity. Nonetheless, the poor key management can compromise the data. Thus, there is a need to deal with this problem by using separate key management technique. For this, the paper suggests to use the algorithm known as Secure Hash Algorithm 3 (SHA-3). This paper presents the key management using the SHA3-256 that have the attack resistance of 2128. Fig. 7 depicts the data security proposed model using AES encryption and SHA-3 


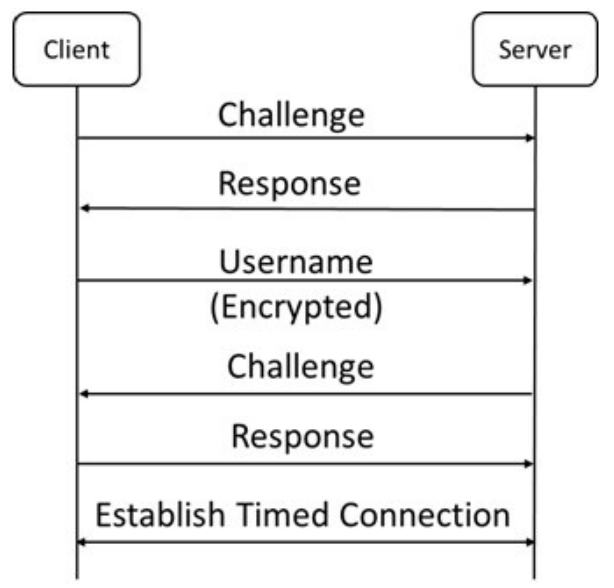

FIG. 5.3. Proposed Client-Server Authentication
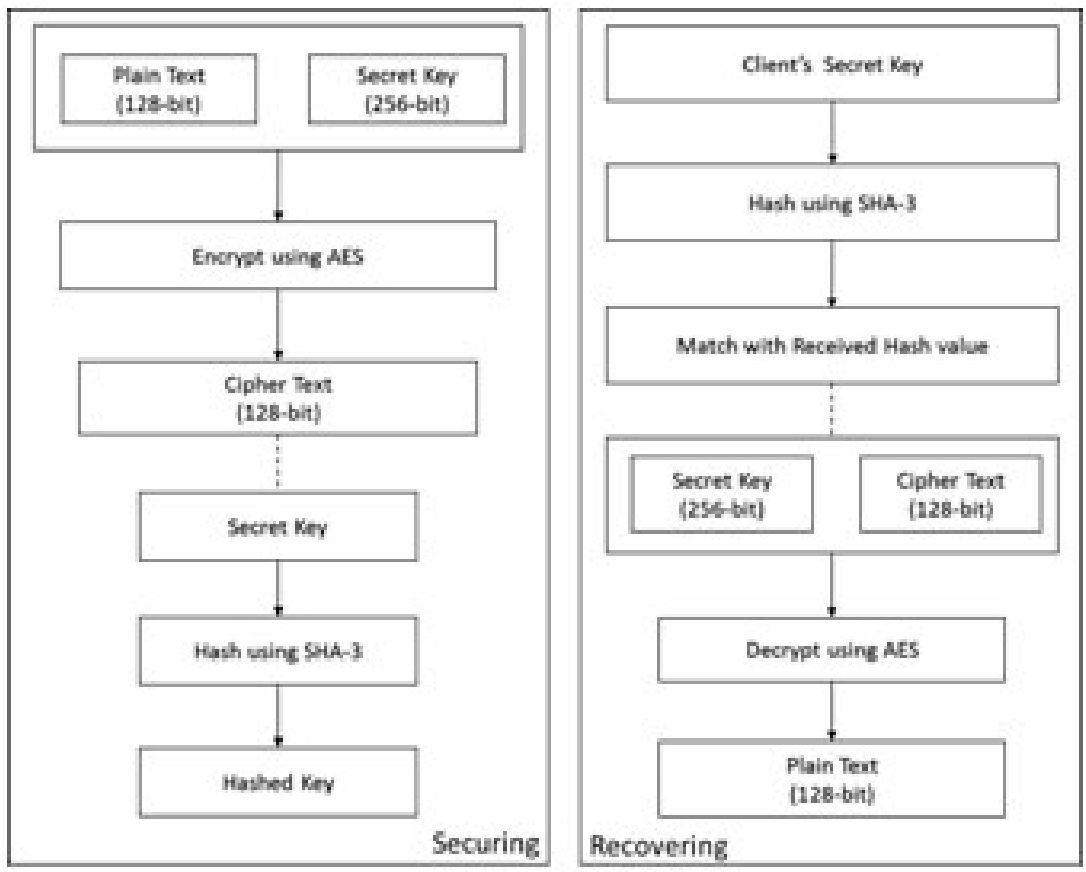

FIG. 5.4. Data Security Proposed Model using AES and SHA-3

hashing technique. The key used in AES for ciphering the text is of 256-bit. The size of block for SHA-3 is 256 bits. The data securing is done by first encrypting the plain text of 128-bit block using a secret key [17]. The cipher text obtained on encryption is the required encrypted data. Now, for key management, the secret key is hashed using the SHA-3 and the hashed key is obtained [18]. In recovering process, the original secret key that client is having, is hashed using the SHA-3. Then the hash value of that key is matched with the hashed value received from the server. In case of match, the key can now be used to decrypt the cipher text using the AES algorithm and the cipher text can be converted back into plain text.

6. Analysis. The proposed model for authentication for client and server side can be analyzed based on the possible common network attacks. The possible common network attacks in this scenario are MITM, DoS and DDoS Attacks. Let us consider the more powerful attack DDoS alone among DoS and DDoS as the DDoS 
is the distributed attack scenario of DoS attack. Therefore, considering the MITM and DDoS attack possibility at every point of exchange of information, the authentication mechanism procedure is analyzed as follows:

Challenge and Response (Client and Server): In the case of DDoS attack, the attacker may try to send meaningless or irrelevant responses for the challenge. But this makes it impossible to continue the attack as the requests will be dropped after the limit is crossed. The limit is set for requests as it is impossible to receive a large number of requests from a client in a particular time limit. Thus, this point is safe from DDoS attack. In case of MITM attack, the attacker clones the client and tries to capture the response sent. Thereafter, there is a possibility that the attacker clones the server and sends the response to the client. But in this at the connection cannot be established as the username step involves encryption that makes it hard to perform MITM as the cloning of client and server along with decryption of username without knowing the actual algorithm and key is a complex process in real life scenario and hard to be implemented due to very high time complexity. As the time is limited for authentication, the attacker fails to perform the attack due to all this complex process. Also, there is a possibility that the attacker tries to fetch the challenge and solve it. In that case, the attacker may get the information of challenge that contains some random string along with hash of password. As the irreversible process is followed, the attacker cannot reverse engineer it, making it impossible to find the actual response value for that challenge thus making it highly safe from MITM attack at these points.

Username: In case of MITM attack, as the username sent is encrypted, so even if the attacker succeeds in capturing the username, it is hard to decrypt it without knowing the encryption algorithm and the value of key. Considering the worst-case scenario, the attacker successfully decrypts the username but in this case, the time limit by that time is already crossed and the process is already completed thereby making it meaningless to decrypt the username. Also, DDoS attack again becomes irrelevant at this point as the meaningless requests sent at this point will not be accepted thus making it highly safe from DDoS and MITM attack at this point.

Established Connection: In case of MITM attack, considering the only possibility that the attacker succeeds in bypassing authentication by somehow cloning the client successfully and establishes connection to server. Nonetheless, the attacker though gets the connection but as the connection is actually a timed connection, thus it will make it impossible to remain into the system after the time gets expired as the challenge and response process is further continued after time gets expired. Also, in case of DDoS attack at the time of established connection, no meaningless request is accepted from client and server side thus making it safe from MITM and DDoS attack at this point.

Thus, this proposed authentication mechanism is highly secure from common network attacks, MITM and DDoS attack. Considering the case of other attacks like phishing and sniffing attack etc., these attacks either comes under these MITM attacks which makes shows that this mechanism is secured from all these attacks. Consider an example of sniffing attack, where the attacker captures the packets being sent. As here the username sent is encrypted so it makes it safe from sniffing attack. Also, as the web page is SSL secured, it makes the webpage safe from phishing attack. Therefore, the mechanism is safe from many network attacks including the most common ones, MITM and DDoS attack.

Now, for the proposed data security model, the securing and recovering mechanism was implemented using the python program and the simulation was done on the system having specifications as: 16GB RAM and Intel i7, 7th Generation Processor on the Windows 10 environment. The simulation results were received for $1 \mathrm{MB}$, $10 \mathrm{MB}, 100 \mathrm{MB}, 1 \mathrm{~GB}$ and $10 \mathrm{~GB}$ file sizes and the running time for the securing and recovering process was recorded. The observed values for the proposed model of recovering and securing process is given in the Table I. Also, the graph depicting the securing and recovering time is shown in the Fig. 6.2. The securing process contains the results for running time that involves the encryption using AES 256-bit key and the generation of the hash value using the SHA-3 256-bit for that key after the encryption process is completed. The recovery process contains the results for the running time that involves generation of hash value for the clients key and matching this hash value with the received hash value from the server followed by decryption of the data using the clients key if the hash value of the clients key matches with the hash value received from the server.

Therefore, the proposed model for securing the data using AES and SHA-3 is safe from most of the known attacks including the password cracking brute force attack, birthday attack, biclique attack, timing attacks etc. as the AES is found to be safe from all these attacks. The only possible vulnerability was that the poor key management can compromise the data encryption. This problem is also solved by the proposed mechanism as 


\begin{tabular}{|c|c|c|}
\hline \multirow{4}{*}{$\begin{array}{l}\text { Proposed } \\
\text { Mechanism } \\
\text { (Encryption } \\
\text { Generation) }\end{array}$} & File Size & Running Time (sec) \\
\hline & 1 Megabyte & 0.0150 \\
\hline & 10 Megabytes & 0.2000 \\
\hline & 100 Megabytes & 1.9300 \\
\hline & 1 Gigabyte & 19.2430 \\
\hline & 10 Gigabytes & 199.5580 \\
\hline \multirow{6}{*}{$\begin{array}{l}\text { Proposed Recovering } \\
\text { Mechanism } \\
\text { (Hash Generation + } \\
\text { Hash Matching + } \\
\text { Decryption) }\end{array}$} & File Size & Running Time (sec) \\
\hline & 1 Megabyte & 0.0220 \\
\hline & 10 Megabytes & 0.1849 \\
\hline & 100 Megabytes & 1.7699 \\
\hline & 1 Gigabyte & 19.0590 \\
\hline & 10 Gigabytes & 128.0299 \\
\hline
\end{tabular}

FIG. 6.1. Observed running time values for proposed model

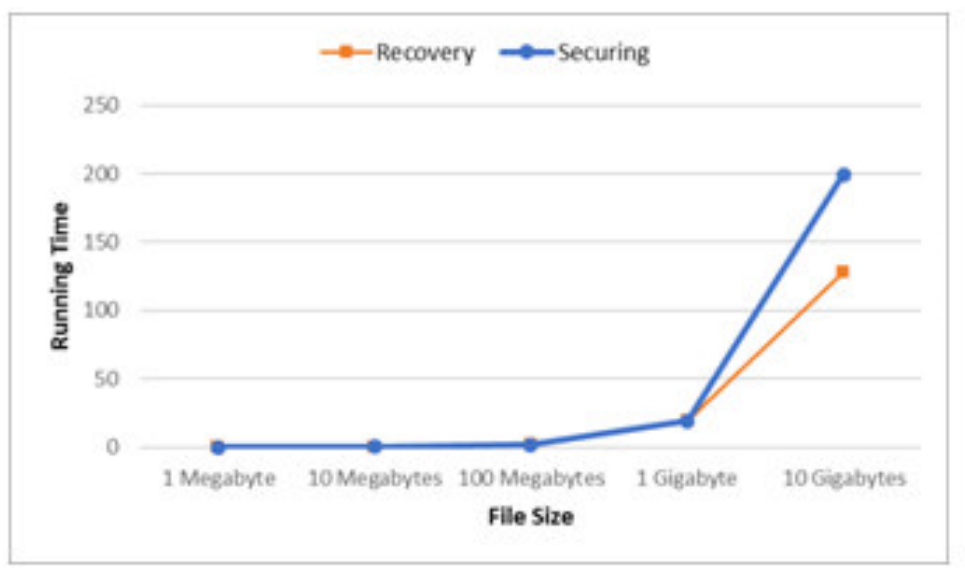

FIG. 6.2. Graph depicting Securing and Recovering Time

the mechanism hashes the key with the help of SHA-3. So, overall the security get user enhanced in terms of authenticity and data security. Thus, due to highly enhanced security during authentication and during accessing the data, it becomes almost impossible for the intruder to fool the security mechanism to get access to the resources and steal information and kept data in the cloud environment.

7. Conclusion. In this paper, a model for security of data in cloud computing environment is proposed that provides a detailed visualization of the security mechanism. The work done in this paper contributes in providing the highest possible security mechanism suitable for public cloud storage with the use of authentication and encryption mechanisms. The proposed model uses the hybrid approach from the existing mechanisms that helps in achieving the highly enhanced security. Future work is to implement further authentication mechanisms such as biometric authentication that includes fingerprint, iris and face recognition techniques as multilevel authentication mechanism. The biometric technique is currently not cost effective considering the cost of highly accurate iris and face recognition. Hence, this paper may also provide a scope for the integration of the biometric authentication into the cloud security mechanism along with the use of proposed authentication mechanism. 


\section{REFERENCES}

[1] M.G. Avram, Advantages and challenges of adopting cloud computing from an enterprise perspective, 7th International Conference Interdisciplinarity in Engineering (INTER-ENG 2013), Volume 12, pp. 529-534, 2014.

[2] Rashmi V. Deshmukh and Kailas K. Devadkar, Understanding DDoS Attack and Its Effect In Cloud Environment, Procedia Computer Science (2015), Vol. 49, pp. 202-210, 2015.

[3] S. Subashini and V. Kavitha, A survey on security issues in service delivery models of cloud computing, Journal of Network and Computer Applications, Volume 34, pp. 1-11, July 2010.

[4] Md. Tanzim Khorshed, A.B.M. Shawkat Ali And Saleh A. Wasimi, A survey on gaps, threat remediation challenges and some thoughts for proactive attack detection in cloud computing.

[5] Madhuri B. Shinde, Design and Implementation of Asymmetric Cryptography Using AES Algorithm, IJARIIE-ISSN(O)2395-4396, Vol-1 Issue-4, pp. 371-378, 2015.

[6] Dr. Ramalingam Sugumar and Sharmila Banu Sheik Imam, Data Security in Public Cloud Storage Environment, International Journal of Engineering Research and Technology (IJERT), ISSN: 2278-0181, Vol. 4 Issue 06, pp. 101-105, June-2015.

[7] Sanjoli Singla And Jasmeet Singh, Survey on Enhancing Cloud Data Security using EAP with Rijndael Encryption Algorithm, Global Journal of Computer Science and Technology Software and Data Engineering, Volume 13, Issue 5, 2013.

[8] G.L. Masala, P. Ruiu, A. Brunetti, O. Terzo and E. Grosso, Biometric Authentication and Data Security in Cloud Computing, Int'l Conf. Security and Management, SAM'15, pp. 9-15.

[9] Nesrine KaAniche and Maryline Laurent, Data Security and privacy preservations in cloud storage environments based on cryptographic mechanisms, Computer Commincations (2017) Vol. 111, pp. 120-141, October 2017.

[10] Minhaj Ahmad Khan, A survey on security issues for cloud computing, Journal of Network and Computer Applications (2016), Vol. 71, pp. 11-29, August 2016.

[11] Sadia Marium, Qamar Nazir, Aftab Ahmed, Saira Ahthasham and Mirza Aamir Mehmood, Implementation of EAP with RSA for Enhancing The Security of Cloud Computing, International Journal of Basic and Applied Sciences , Volume 1, Issue 3, pp. 177-183, 2012.

[12] Atewologun Olumide, Abeer Alsadoon, P.W.C. Prasad and Linh Pham, A Hybrid Encryption model for Secure Cloud Computing, 2015 Thirteenth International Conference on ICT and Knowledge Engineering, pp. 24-32, November 2015.

[13] Babitha M.P And K.R. Remesh Babu, Secure Cloud Storage Using AES Encryption, 2016 International Conference on Automatic Control and Dynamic Optimization Techniques (ICACDOT), pp. 859-864, September 2016.

[14] Sanjoli Singla And Jasmeet Singh, Cloud Data Security using Authentication and Encryption Technique, International Journal of Advanced Research in Computer Engineering and Technology (IJARCET), Volume 2, Issue 7, pp. 2232-2235, July 2013.

[15] Mrs. S. M. Barhate And Dr. M. P. Dhore, User Authentication Issues In Cloud Computing, IOSR Journal of Computer Engineering (IOSR-JCE), Volume 4, pp. 30-35, 2016.

[16] Shakeeba S. Khan And R.R. Tuteja, Security in Cloud Computing using Cryptographic Algorithms, International Journal of Innovative Research in Computer and Communication Engineering, Volume 3, Issue 1, pp. 148-154, January 2015.

[17] Zaid Kartit, Applying Encryption Algorithm to Enhance Data Security in Cloud Storage, Advances in Ubiquitous Networking. Lecture Notes in Electrical Engineering, Vol 366, pp. 141-154, Springer, Singapore.

[18] J R Ngnie Sighom, Pin Zhing And Lin You, Security Enhancement for Data Migration in the Cloud, Future Internet, Volume 9, Issue 3, pp. 1-13, June 2017.

Edited by: Rajkumar Rajasekaran

Received: Jul 24, 2018

Accepted: Dec 4, 2018 


\title{
CLOUD BASED DYNAMIC COURSE SELECTION FRAMEWORK USING NETWORK GRAPHS WITH TERM DIFFICULTY ESTIMATION*
}

\author{
JASEM M. ALOSTAD †
}

\begin{abstract}
The system developed in this paper uses a cloud based technology to implement and design a software as a service (SAAS) application for adaptive course selection and term difficulty estimation for a networked curriculum. The choice of courses in every term is completely in the hands of the students who enroll for a particular program in Universities. The order of courses taken in every term is ad hoc due to different factors like student interests, uncertainty about the student pass rates, frequent changes in admission policies and curriculum requirements. However, this choice of course plays a vital role in students graduating in time from the university. In this paper, we analyze student success ratios in terms of time to graduation. To illustrate the designed models, data from different colleges of the Public Authority of Applied Education and Training (PAAET), Kuwait, is used. Graph-based complex networks are used for analyzing the courses and how crucial they are. The difficulty levels of courses are estimated based on the institutional data from spring 2013 to fall 2016 and term difficulties are estimated based on the courses chosen. This work presents a robust framework which is adaptable to the courses chosen by the students and the ease of flow of students through the curriculum with the aim of improving the universitys graduation rate.
\end{abstract}

Key words: Parallel processing, Data Analytics, Cloud computing, Graph Theory, Student Success Ratio, Time to Graduation, Course Cruciality, Course Difficulty

AMS subject classifications. 68M14, 90C35

\section{Introduction.}

1.1. Background. Prediction and investigations on academic performance and the factors affecting student success and the endurance of students are topics of utmost importance in higher education [18]. Academic performance is a crucial factor in analyzing higher education levels and predicting other important job outcomes like performance in the job hired for and salary predictions [19]. The success ratio is an important metric for the students and the university. The success of a student has many definitions, varying from the grades to self-improvement [7, 8]. Most of the studies from the literature indicate student graduation in time as the most important success metric. From the perspective of the university, and especially for public universities, factors like graduation, student retention rate and time to degree are essential for the so called performance funding of the universities from the state. Generally, the final grades obtained by the student are used for analyzing the performance of a student.

The graduation rate of students depends on two factors: institutional and pre-institutional factors [11, 12]. The students perspective on the graduation rate depends on pre-institutional factors like High school performance, the demographic status of the students, socio-economic data and some of the institutional factors include the guidelines and policies of the university, tutoring, advisory arrangements and the competence of the instructors $[13,14]$. The success framework of students is shown in Fig.1.1 as discussed in [10]. Prediction and progression results are used in [17] educational data mining to analyze student performance.

In this paper we are considering the optimal course choice models for The Public Authority of Applied Education and Training (PAAET), a higher education institute in Kuwait, offering a range of programs through its various colleges across the country. There are five different colleges and two campuses differentiated gender wise, for each college. PAAET is considered as one of the largest institutes in the Middle East, taking student enrollment as a factor in the measurement. Every term approximately 40000 students are admitted to different colleges in PAAET. There are 5 different colleges, namely College of Basic Education, College of Business Studies, College of Health Sciences, College of Nursing and College of Technological Studies under PAAET. Of all the colleges, the College of Basic Education has more number of students enrolled each term. BA0106, a four year degree program of the college of Basic Education, PAAET, is considered for further processing, analysis and discussion in this paper.

\footnotetext{
*This work was supported by The Public Authority of Applied Education and Training, Kuwait.

†College of Basic Education, The Public Authority of Applied Education and Training (PAAET), Kuwait,(jm.alostad@paaet.edu.kw).
} 


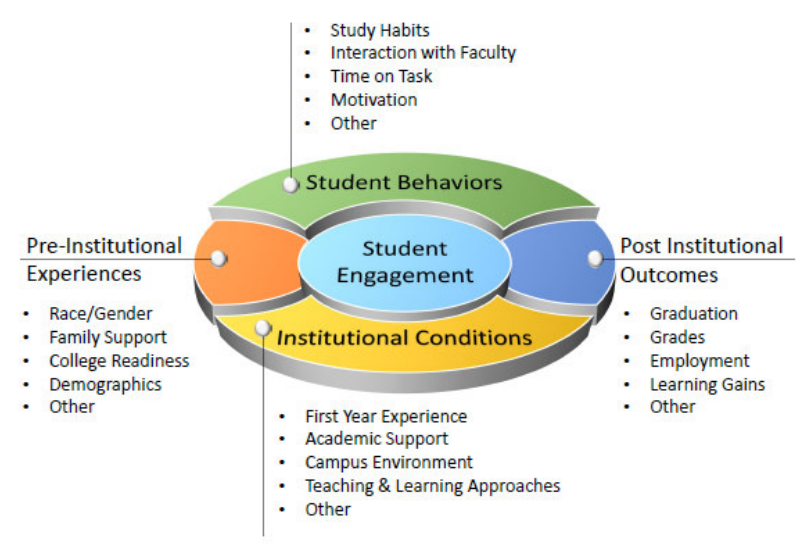

FIG. 1.1. Student success framework.

1.2. Problem Statement. Student graduation rate is one of the most important metric in evaluating the rank of a College or University. Universities where the success ratio is large, has largest enrollments in years to come. E-Advisor is a web based application providing online guidance to the students of PAAET. Here guidelines are given in the direction of finding appropriate disciplines so they can succeed by graduating in-time. The e-advisor system provides the detailed information of the different colleges in PAAET, their major courses, the success rate in each of the course in the previous years and the rules and regulations of the Authority. The current system lacks options for guiding student about the subjects that has to be chosen in future semesters. Hence, for the objective of increasing the Graduation rate of the student, choice of courses in every term is taken into consideration in this work. The proposed solution acts as an advising system for the students. This solution helps students who are below average and who are unaware of being expelled from college for reaching the maximum allowed semesters in their period of graduation. They system alerts the students as early in his/her 3 rd semester about his delay in graduation and time to graduation.

1.3. Proposed Solution. A critical institutional factor that is often glossed over is the order of course choices every term i.e. the structure of the curriculum, which is linked with the program which a student has selected. This is one of the most relevant perspectives in understanding the academic performance of a student. Hence, in this paper we analyze the course choices in every term and identify the most crucial courses in the curriculum. The key point is failure in such crucial courses or if the courses are not taken according to schedule this will directly lead to delay in graduation. This analysis is carried out program wise. In this process we developed an adaptable framework which is used to guide the student on choosing courses every term so that he is not delayed in graduation.

Contributions: In this paper the problem of student success ratio is addressed in terms of his/her graduation in-time from the university. The proposed algorithm incorporates the following unique features

1. A network graph for the entire courses in a particular program is constructed for the algorithm implementation.

2. Course prerequisites, co requisites and level of the courses are taken into consideration.

3. An important feature for student success; choice of courses in every allowed term by students in order to identify their time to graduation is considered. As an advisory system, our algorithm gives an optimal set of courses for a student for a term in order to graduate in time.

4. A dynamic course selection algorithm is developed which displays the optimal choice and allows a student to decide the courses on his own. Depending on the courses chosen; the algorithm dynamically recomputes the optimal choice of courses from the next term and an alert is generated if the student is expected to exceed the number of allowed terms.

5. The difficulty of every course in the program is identified using the institutional data from the years 
2013 to 2016.

6. With the calculated course difficulty, term difficulty is computed and future term difficulty is also predicted with the current selection of courses.

Organization: The rest of the paper is organized as follows. Section 2 gives a brief review of the literature considering the work done earlier in this field. In section 3 the proposed adaptive course selection framework is presented in detail, considering the static and dynamic selections. Section 4 presents details about the difficulty analysis of every course in the chosen program. Section 5 discusses the numerical results obtained with the sample data and Section 6 concludes the work.

2. Related Works. A student performance analysis system presented in [1] uses students' grades to classify students into different clusters. The performance of a student on a particular course is analyzed and predicted using data mining techniques. A case study on the Educational data mining techniques is presented in [2], where classification based data mining algorithms are presented for analyzing the student performance. Similarly in an exhaustive study in the field of educational data mining [3], the prediction algorithms used for student performance analysis and their comparison is presented.

Abeer and Elaraby [4] used the demographic information about students, including their behaviour and activities, with rules for classification and predicted the performance of students. The approximate grades of the students are predicted, which helped in improving the student performance on future courses.

An association rule based performance prediction and improvement in higher education is proposed in [6]. The work presented in [5] is based on knowledge mining from the educational data using a decision tree and identifying the students with low performance ranges and it suggests improvements in their learning methodology.

In [15] different metrics for measuring student success are analyzed. The five most important metrics included in the discussion are: retention rates, graduation rates, time to completion, academic performance, and tracking educational goals. The graduation rate and the retention rate are closely related and the factors affecting both the metrics are almost the same. The most important factor to be considered in these two metrics is the unpredictability in students' paths. A prediction model for identifying the social, economic and psychological factors a student is facing in his adolescence is analyzed in [16]. The model takes data balancing, dimensionality reduction, discretization and normalization to pre process the data and predictor construction.

In all the work proposed in the literature in the direction of analysis of student success or performance analysis recent data mining techniques have been used to analyze the performance of the students and identify good or bad performance, enabling the teacher and the student himself to explore different ways to improve performance in future terms. We recognize an important factor in student success as the courses taken during his period of study. Difficulty analysis is done for different courses in [22], where different data such as high school GPA, ICT scores, demographic data and the grades obtained by the students in the previous years are taken into consideration. The institutional data plays a major role in deciding the difficulty of the courses conducted in the university. Hence, in this work we build upon this to identify the static and dynamic term difficulty as a warning or information for students, providing awareness about the level of his course. Curriculum design patterns and applications are explained in detail in [20,21], which is the basis of the work presented in this paper. The difficulty level and how crucial courses are considered to be should be known before choosing a course. Hence, in this work we present a framework for providing an optimal choice of courses for a particular term for a student so that the success rate of the student increases, enabling a high graduation rate from the university.

3. Adaptive Course Selection Framework. The proposed course selection framework is explained in detail in what follows. The network construction of courses in the curriculum of a program is described first and the algorithm for deciding the optimal course choice is discussed later.

3.1. Course Network Construction. As a measure of a students success, graduating in time from the university is considered as an important institutional factor. For a student to graduate in time the requirement is completion of the courses and earning the required credits. The order of course choice plays an important role in time to graduation as the courses in the curriculum are sometimes not dependent but some of the courses has pre-requisites which have to be completed before taking them up. Hence, in this paper the curriculum 
associated with a particular degree program is taken and the choice of courses in different terms is identified using the course selection algorithm. Due to the nature of course networks, graph theory and complex network analysis is used to find out the most crucial courses in the curriculum mathematically. This calculation of how crucial a course is can guide the student in deciding when to choose a particular course in order to avoid delay in graduation.

The curriculum graphs are constructed using graph theory. Here the courses in the curriculum are represented as the nodes and two nodes are connected if the course has a direct prerequisite. Hence, the course network is represented as an $N x N$ adjacency matrix $\mathrm{M}$, where $\mathrm{N}$ is the number of courses in a program. If there is a vertex between two nodes the corresponding entry in the adjacency matrix $M_{i j}=1,0$ otherwise .

We define two properties, the Delay factor and blocking factor for each course. The Delay and Blocking factor is 0 for courses that are independent, i.e courses without any prerequisites. Hence, for the courses with prerequisites the length of the longest path from a node to its leaf node is the delay factor $D f_{i}$. The delay factor identifies courses that drive a student to being at risk if it is not finished on time. Connectivity of a node is considered in calculating the blocking factor of the node $B f_{i}$. The Blocking factor identifies the courses that are prerequisites for a large number of courses. If such a course is not completed at the right time a student may be blocked from the follow-on courses, which means negative progress towards graduation. The total number of nodes connected to a particular node gives $B f_{i}$ of a node. $B f_{i}$ is defined by equation 3.1:

$$
B f_{i}=\sum_{j} n_{i j}
$$

where $n_{i j}=1$ if there is a path from $i$ to $j, 0$ otherwise. Cruciality of a node is calculated as the sum of the blocking factor and the delay factor. Cruciality $C_{i}$ is given in equation 3.2 :

$$
C_{i}=B f_{i}+D f_{i}
$$

Hence cruciality factor of a course plays a vital role in deciding when to take a particular course. Since delay and blocking both are taken into consideration, a high crucial course implies that the course has to be given preference and taken in appropriate time failing which results in delay in graduation.

3.2. Static Course Preference Algorithm. The Algorithm for course selection is given in Fig.3.1. Let Cr be the number of courses in the curriculum, $\mathrm{T}$ be the number of terms, assign the credit points to all the

\section{Algorithm 1 Static Course Preference}

Let $\mathrm{N}$ be number of courses in each term

Initialize $t=1$

while $\mathrm{Cr}$ is not empty and $t<T$ do

1. Calculate the cruciality of the courses listed in the curriculum

2. Sort the list of courses in ascending order of course levels and descending order of the cruciality factor

3. Select the first $\mathrm{N}$ courses from the list so as to satisfy the limits in the sum of credits that can be registered.

4. Select the courses such that there are no prerequisites.

5. Check if any of the selected coursehas co-requisites. if true the co-requisites and its corresponding prerequisites if any has to be processed first.

6. These courses will fill term .

7. Remove the selected courses

from $\mathrm{Cr}$ and $\mathrm{M}$

end while

8. $t=t+1$

FIG. 3.1. Course Preference algorithm 


\section{Algorithm 2 Dynamic Course Preference}

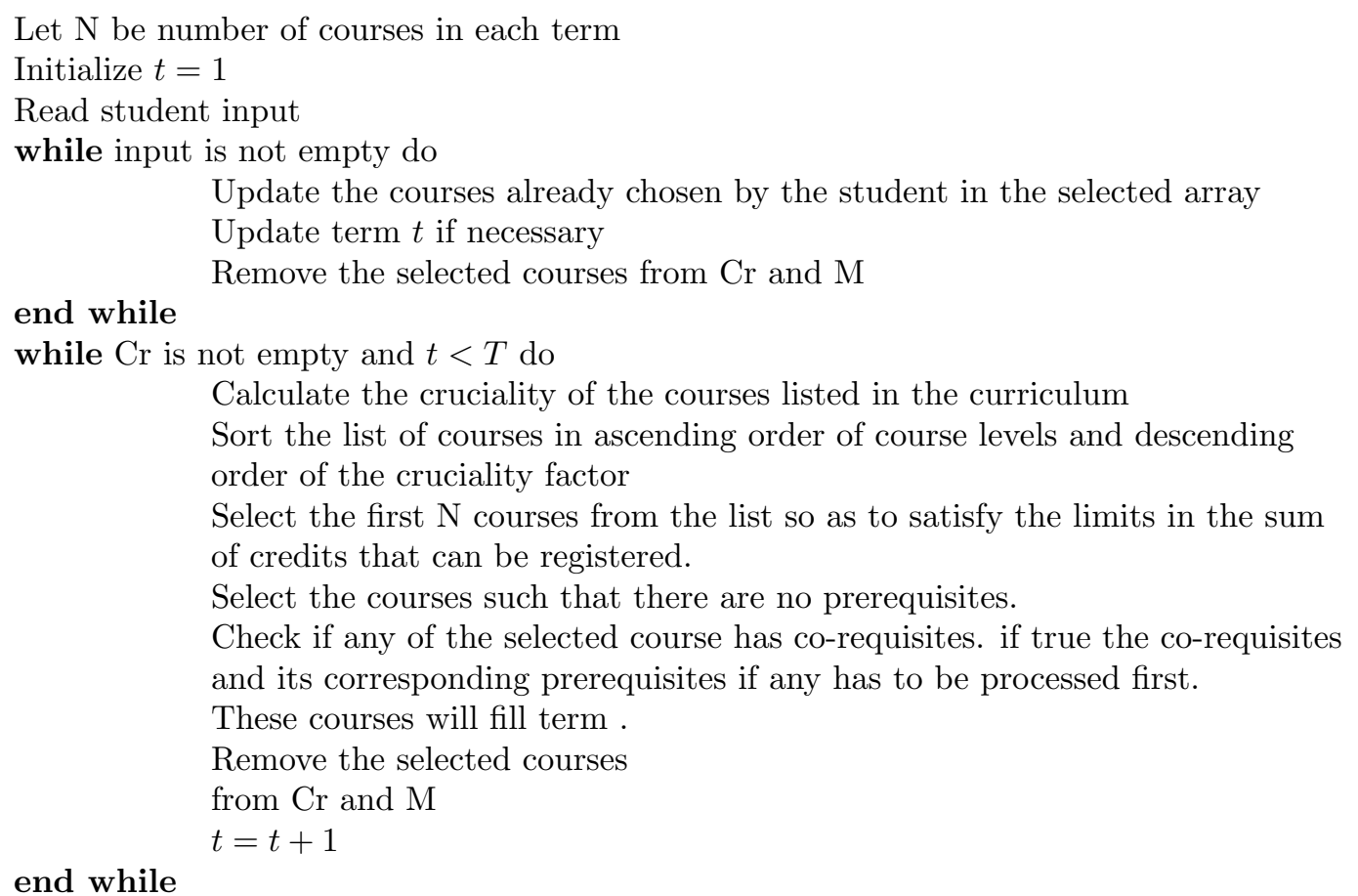

FIG. 3.2. Dynamic Course Preference algorithm

terms as mentioned, and let $\mathrm{M}$ be the adjacency matrix representing the course and the prerequisite relation. The algorithm generates an ordered list of courses term-wise for the student, so that he can graduate in time within the allowed terms.

3.3. Dynamic Course Preference algorithm. The Dynamic course preference algorithm allows a student to choose a course on his own (which may or may not be from the output of the static algorithm), and for the rest of the terms the ordered courses list is displayed to the student. The number of terms required to graduate with the current choice of courses is also displayed in the output of the algorithm. The algorithm is shown in Fig. 3.2.

4. Course Difficulty: Institutional Data Analysis. Institutional Data for 8 terms from spring 2013 to fall 2016 are analyzed to identify the difficulty of courses. The difficulty estimations are done based on the previous grades. Mean and standard deviations are calculated for the grade distributions for all the courses for each of the 8 terms. Some of the courses are not taught in some terms and, hence, the means of the courses for those particular terms are not available. The rank for each course in each term is calculated and as a sample the ranks of 25 courses are shown in Table 4.1. The ranks indicate there is a considerable amount of variation in grade distribution in every term.

In order to ensure stability of course difficulty over time, mean of the grade values are ranked. In spite of ranking the mean of the grade values there are difference in some mean grades because not all the courses are taught in all the semesters, it depends on the choice of the students. To analyze these variations further, correlation among the 8 terms are calculated using pearson's $R$ method. On examining the correlation coefficients supports the general idea that the grade distributions are consistent across several semesters. The calculated correlations are plotted in the correlation plot shown in Fig.4.1. The correlation matrix is shown table 4.2. The minimum correlation exists between the terms Spring2016 and Fall 2016, on examining the mean grade values of these terms reveals that several courses had their extreme values in these semesters. The correlation graph 
TABLE 4.1

Rank of Courses (mean grades) in Various Terms

\begin{tabular}{|c|c|c|c|c|c|c|c|c|}
\hline Courses & S13 & F13 & S14 & F14 & S15 & F15 & S16 & F16 \\
\hline X101.102 & 5 & 6 & 6 & 29 & 47 & 9 & 1 & NA \\
\hline X102.101 & 3 & 3 & 2 & 1 & 6 & 2 & 4 & 13 \\
\hline X102.102 & 4 & 5 & 4 & 6 & 10 & 3 & 8 & 7 \\
\hline X103.105 & 1 & 1 & 3 & 4 & 5 & 5 & 2 & 3 \\
\hline X103.115 & 2 & 2 & 1 & 2 & 3 & 1 & 3 & 4 \\
\hline X106.101 & 9 & 13 & 11 & 12 & 14 & 4 & 13 & 11 \\
\hline X106.104 & 10 & 4 & 5 & 11 & 7 & 7 & 10 & 8 \\
\hline X107.104 & 55 & 10 & 48 & 25 & 54 & 51 & 40 & 45 \\
\hline X107.121 & 54 & 54 & 52 & 50 & 46 & 46 & 38 & 46 \\
\hline X107.124 & 39 & 35 & 39 & 36 & 20 & 32 & 35 & 38 \\
\hline X107.131 & 42 & 41 & 50 & 47 & 31 & 34 & 31 & 20 \\
\hline X107.134 & 51 & 16 & 18 & 22 & 8 & 37 & 22 & 26 \\
\hline X107.154 & 26 & 32 & 35 & 32 & 18 & 8 & 42 & 12 \\
\hline X107.161 & 45 & 33 & 43 & 31 & 36 & 36 & 30 & 47 \\
\hline X107.171 & 52 & 47 & 45 & 33 & 41 & 40 & 29 & 35 \\
\hline X107.194 & 41 & 27 & 26 & 35 & 13 & 21 & 5 & 32 \\
\hline X107.214 & 33 & 38 & 46 & 45 & 23 & 30 & 39 & 17 \\
\hline X107.244 & 28 & 34 & 41 & 38 & 19 & 24 & 25 & 25 \\
\hline X107.251 & 48 & 48 & 54 & 26 & 21 & 22 & 37 & 30 \\
\hline X107.254 & 35 & 36 & 22 & 43 & 22 & 42 & 6 & 48 \\
\hline X107.264 & 50 & 42 & 21 & 42 & 26 & 23 & 9 & 44 \\
\hline X107.331 & 53 & 51 & 53 & 51 & 51 & 49 & 48 & 31 \\
\hline X107.341 & 49 & 53 & 42 & 46 & 43 & 33 & 32 & 43 \\
\hline X107.342 & 43 & 40 & 40 & 49 & 37 & 48 & 45 & 40 \\
\hline X107.372 & 44 & 52 & 44 & 18 & 27 & 20 & 43 & 23 \\
\hline
\end{tabular}

TABLE 4.2

Correlation of mean grades for 8 terms

\begin{tabular}{|c|c|c|c|c|c|c|c|c|}
\hline & S13 & F13 & S14 & F14 & S15 & F15 & S16 & F16 \\
\hline S13 & 1.0000000 & 0.77334600 & 0.86108600 & 0.7729660 & 0.6197429 & 0.7623634 & 0.6105081 & 0.6783107 \\
\hline F13 & 0.7733460 & 1.00000000 & 0.85515533 & 0.8075693 & 0.6784647 & 0.7075601 & 0.6590922 & 0.6438203 \\
\hline S14 & 0.8610860 & 0.85515533 & 1.00000000 & 0.8437116 & 0.7005898 & 0.7777775 & 0.6910246 & 0.6487445 \\
\hline F14 & 0.7729660 & 0.80756928 & 0.84371157 & 1.0000000 & 0.6835913 & 0.8127427 & 0.6042601 & 0.7371457 \\
\hline S15 & 0.6197429 & 0.67846468 & 0.70058981 & 0.6835913 & 1.0000000 & 0.6993695 & 0.7079145 & 0.5426127 \\
\hline F15 & 0.7623634 & 0.70756014 & 0.77777746 & 0.8127427 & 0.6993695 & 1.0000000 & 0.5991665 & 0.7315858 \\
\hline S16 & 0.6105081 & 0.65909216 & 0.69102459 & 0.6042601 & 0.7079145 & 0.5991665 & 1.0000000 & 0.5144824 \\
\hline F16 & 0.6783107 & 0.64382030 & 0.64874449 & 0.7371457 & 0.5426127 & 0.7315858 & 0.5144824 & 1.0000000 \\
\hline
\end{tabular}

indicates that there is no correlation in the opposite direction. Deviations from normality are also identified using the Shapiro-Wilk's test. The sample normality plots for fall 2014 and spring 2016 shown in Fig.4.2 show no traces of lack of normality. The $\mathrm{p}$ values range from 0.1895 to 0.9958 .

\section{Numerical Results and Discussions.}

5.1. Static Course Choices. To showcase the above mentioned process we have selected the courses of the BA0106 program of the College of Basic Education, PAAET. There are 90 courses in total and these are categorized as mandatory courses for the Major, Elective courses, and General courses. Of the three categories the student has to choose courses totaling 130 credits in 4 years to graduate. The number of credits a student can register for a term is restricted to 18 in fall and spring and 7 in summer. The summer terms are optional for the students. The entire set of courses in the program is represented as the network shown in Fig.5.1. The nodes in the network represent the courses. Courses are named as a combination of the department id and the course id. The courses without any prerequisites are represented as nodes without any edges and the courses connected by prerequisites have edges between them.

The cruciality factor is calculated for the courses in the program according to equation 3.1. The most 


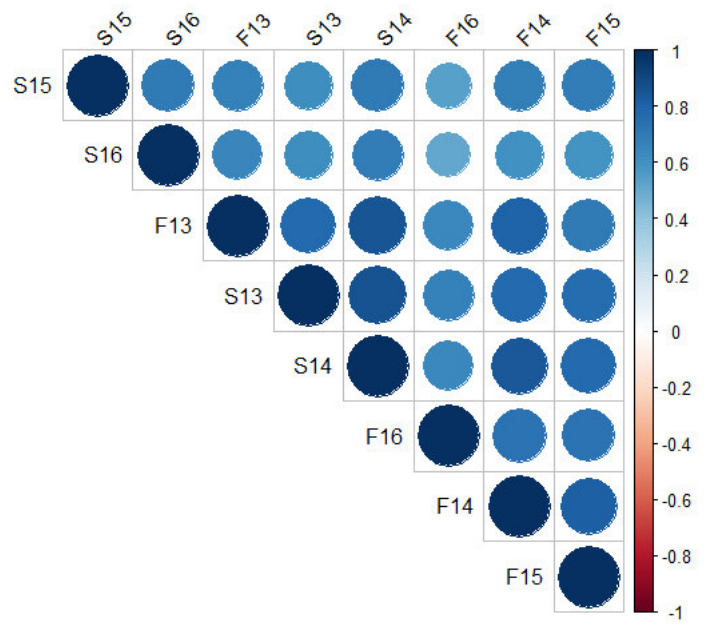

FIG. 4.1. Correlation among the 8 terms

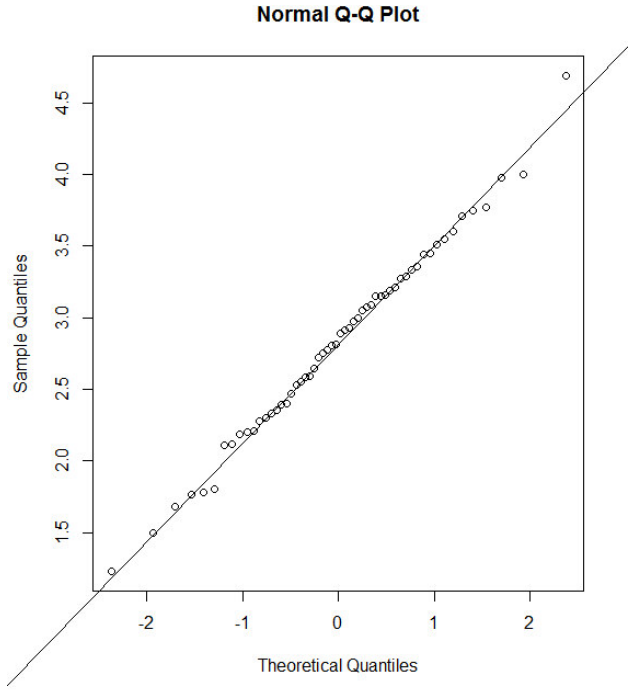

(a) Case I

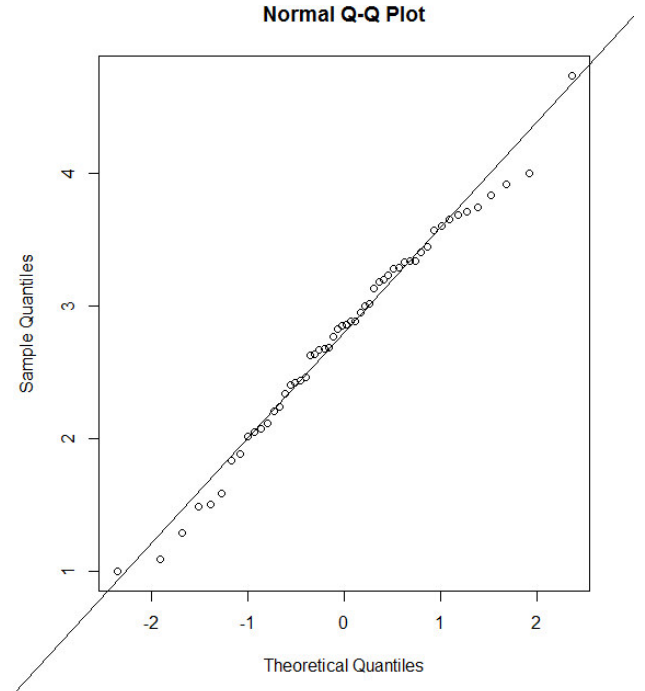

(b) Case II

FIG. 4.2. Sample normality plots for Fall 2014 and Spring 2016

crucial 10 courses are shown in Table 5.1.

The static course preference algorithm is executed on the network constructed. The different courses that a student has to choose in order to graduate in time are the output generated from the algorithm. These lists of courses are shown in Table 5.2. The co-requisites and prerequisites are handled optimally and the course levels are also taken into consideration. The total number of credits earned is 130 .

5.2. Adaptive course choices. The actual choice of courses is in the hands of the students. Hence, we have also presented a dynamic version of the algorithm which initially displays the optimal choice of courses and reads the input file containing the actual courses chosen by the student and, based on the courses chosen; the set of optimal choices for the upcoming terms is displayed to the student. The algorithm also displays the number of terms a student will actually need for graduation with his current choice of courses. Based on the output the student may choose to take one or more summer terms as per his requirements. As an illustration 


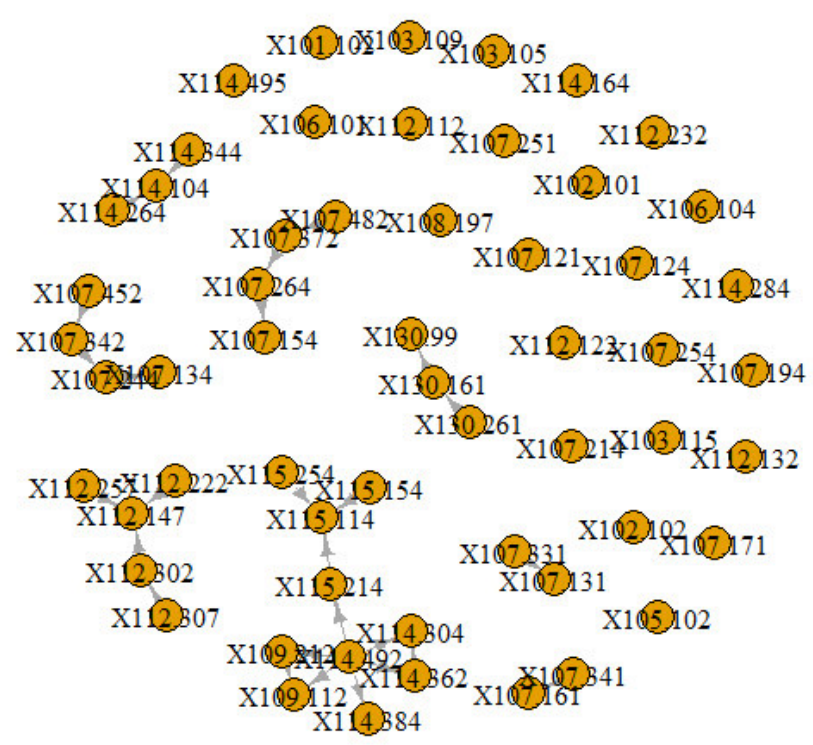

FIG. 5.1. Course Network Structure of courses and Prerequisite courses

TABLE 5.1

10 Most crucial courses in the Curriculum

\begin{tabular}{|c|c|c|c|}
\hline Course & Connectivity & Delay & Cruciality C \\
\hline X114.492 & 6 & 3 & 9 \\
\hline X115.114 & 5 & 3 & 81 \\
\hline X109.112 & 2 & 4 & 6 \\
\hline X109.212 & 2 & 4 & 6 \\
\hline X114.304 & 2 & 4 & 6 \\
\hline X114.362 & 2 & 4 & 6 \\
\hline X114.384 & 1 & 4 & 5 \\
\hline X115.154 & 1 & 4 & 5 \\
\hline X115.254 & 1 & 4 & 5 \\
\hline X115.424 & 1 & 4 & 5 \\
\hline
\end{tabular}

of the dynamic course selection algorithm, for the above mentioned program BA0106, the courses chosen by a student as shown in Table 5.3 is given as the input. For the given input the choice of courses to be chosen is given in Table 5.4. It can be observed from Table 5.4 that the first two term data is given from the actual courses chosen by the student and for the rest of the terms the courses to be chosen are displayed in Table 5.4. The number of terms in Table 5.4 is given as 9, hence, the student is expected to exceed one term over the allotted number of terms. The student can plan for a summer term in order to graduate in time.

Fig. 5.2 shows the dynamic course network that adapts to the courses chosen by the student. The 8 subfigures of Fig.5.2 present the structure of the network after choosing the courses. It represents the remaining courses in the curriculum of the program after every term. It can be observed from the figure that the remaining courses in Fig.5.2.(h) are the courses chosen in the $9^{\text {th }}$ term by the student as shown in Table 5.4. It can also be inferred from Table 5.4, that the course levels are not maintained in the dynamic selection. The reason is the input decided by the student is random without considering the course levels, with the random input the cruciality, co-requisites and prerequisites are considered since the input does not follow the level.

5.3. Difficulty Estimation for Courses. The course difficulties are estimated based on the institutional data, i.e the grade distributions. The careful examination of the correlation coefficients supports the general perception that the distribution of grades is highly consistent across semesters. The Shapiro-Wilk's test and the 
TABLE 5.2

Term wise courses

\begin{tabular}{|c|c|c|c|c|c|c|c|c|}
\hline Term 1 & X130.99 & X102.101 & X106.101 & X102.102 & X101.102 & X114.103 & X114.104 & X106.104 \\
\hline Term 2 & X107.104 & X103.105 & X109.112 & X112.112 & X115.114 & X103.115 & X107.121 & \\
\hline Term 3 & X112.122 & X107.124 & X107.131 & X112.132 & X107.134 & X112.147 & X115.154 & X107.154 \\
\hline Term 4 & X130.161 & X107.161 & X114.164 & X107.171 & X107.194 & X108.197 & X109.212 & X115.214 \\
\hline Term 5 & X107.214 & X112.222 & X112.232 & X107.244 & X107.251 & X115.254 & X107.254 & X112.257 \\
\hline Term 6 & X130.261 & X107.264 & X114.284 & X112.302 & X114.304 & X107.331 & X107.341 & \\
\hline Term 7 & X112.307 & X107.342 & X114.344 & X114.362 & X107.372 & X114.384 & X114.464 & \\
\hline Term 8 & X114.492 & X114.495 & X107.482 & X107.452 & & & & \\
\hline
\end{tabular}

TABLE 5.3

Student Course Choice input

\begin{tabular}{|c|c|c|}
\hline Term & Dept_code & Course_Number \\
\hline 1 & 114 & 384 \\
\hline 1 & 103 & 105 \\
\hline 1 & 115 & 114 \\
\hline 2 & 114 & 284 \\
\hline 2 & 101 & 102 \\
\hline 2 & 114 & 304 \\
\hline 2 & 108 & 197 \\
\hline
\end{tabular}

Pearson's coefficients determine the stability of average grades. It appears to be a reasonable assertion that the grade distribution of individual courses does not change considerably from term to term. To be certain some variations indeed do occur in a minimal number of courses.

Nevertheless, the average grades appear to be stable enough to be used for estimating course difficulties. Hence, the eight term grade distributions are combined into one set of 57 grade distributions. Hence, average grades and their standard deviation are calculated, a sample of the first 25 courses is shown in Table 5.5 and these means are ranked in ascending order to identify the most difficult course.

The difficulty levels of the courses are represented in a heat map in Fig. 5.3. The colors vary from dark red to yellow for courses that are more difficult to easy.

With these course difficulty levels we also calculate the term difficulty by averaging the difficulty level of courses chosen for a particular term. Term difficulty is calculated both for the static course choices as in Fig. 5.4.(a) and dynamic course choices as in Fig. 5.4.(b). In the dynamic algorithm the term difficulty of the future terms is also predicted. This is given as advice to the student so that the courses can be chosen according to his convenience.

In another direction, this dynamic course preference algorithm can be used for identifying the students who will not graduate with the maximum allowed terms for graduation. Every program has the designated number of terms for a student to graduate in-time from the university. There is also a maximum allowed term for graduation beyond which a student can never graduate. Such a category of student who is anticipated not to graduate is identified early by the proposed algorithm, so that the management can decide upon such students in their early terms instead of allowing them for the maximum terms and terminating them from the university. On the other hand, a student is also given an opportunity to decide on his future in his early college life.

The cruciality analysis for different courses can be expanded department-wise and college-wise in order to identify the department with more crucial courses and the college with the most crucial departments. This data can be used further in allotting grade ranges for different subjects. The future direction of this work is allocating grade ranges for subjects within the curriculum. The grade ranges may vary based on the cruciality of the course. Less crucial courses have different ranges of values for grades compared to more crucial courses. The outcome GPA, which counts in factors like employment and higher education, will there for be fair to all students, be it a student who graduated studying less crucial courses or more crucial courses.

6. Conclusion and future enhancement. In this paper, network analysis and graph theory have been used to propose a framework to understand the structure of University courses by calculating the cruciality factor. Using this framework, the course selection algorithm shows the optimal choice of courses to be selected 
TABLE 5.4

Student Course Choice input

\begin{tabular}{|c|c|c|c|c|c|c|c|c|}
\hline 1 & $\mathrm{X} 114.384$ & $\mathrm{X} 103.105$ & $\mathrm{X} 115.114$ & & & & & \\
\hline 2 & $\mathrm{X} 114.284$ & $\mathrm{X} 101.102$ & $\mathrm{X} 114.304$ & $\mathrm{X} 108.197$ & & & & \\
\hline 3 & $\mathrm{X} 109.112$ & $\mathrm{X} 114.362$ & $\mathrm{X} 112.147$ & $\mathrm{X} 115.154$ & $\mathrm{X} 115.254$ & $\mathrm{X} 107.134$ & $\mathrm{X} 107.154$ & $\mathrm{X} 114.104$ \\
\hline 4 & $\mathrm{X} 109.212$ & $\mathrm{X} 107.244$ & $\mathrm{X} 107.264$ & $\mathrm{X} 115.214$ & $\mathrm{X} 112.222$ & $\mathrm{X} 112.257$ & $\mathrm{X} 112.302$ & $\mathrm{X} 114.344$ \\
\hline 5 & $\mathrm{X} 114.492$ & $\mathrm{X} 114.495$ & $\mathrm{X} 107.372$ & $\mathrm{X} 107.342$ & $\mathrm{X} 112.307$ & $\mathrm{NA}$ & $\mathrm{NA}$ & $\mathrm{NA}$ \\
\hline 6 & $\mathrm{X} 107.452$ & $\mathrm{X} 107.482$ & $\mathrm{X} 130.99$ & $\mathrm{X} 107.121$ & $\mathrm{X} 107.131$ & $\mathrm{X} 107.161$ & $\mathrm{X} 107.171$ & $\mathrm{X} 107.251$ \\
\hline 7 & $\mathrm{X} 130.161$ & $\mathrm{X} 112.112$ & $\mathrm{X} 107.331$ & $\mathrm{X} 107.341$ & $\mathrm{X} 107.124$ & $\mathrm{X} 107.194$ & $\mathrm{X} 107.214$ & $\mathrm{X} 107.254$ \\
\hline 8 & $\mathrm{X} 130.261$ & $\mathrm{X} 102.102$ & $\mathrm{X} 106.104$ & $\mathrm{X} 103.115$ & $\mathrm{X} 112.132$ & $\mathrm{X} 114.164$ & $\mathrm{X} 112.232$ & NA \\
\hline 9 & $\mathrm{X} 102.101$ & $\mathrm{X} 106.101$ & $\mathrm{X} 114.264$ & $\mathrm{X} 105.102$ & $\mathrm{X} 112.122$ & $\mathrm{X} 103.109$ & $\mathrm{NA}$ & NA \\
\hline
\end{tabular}

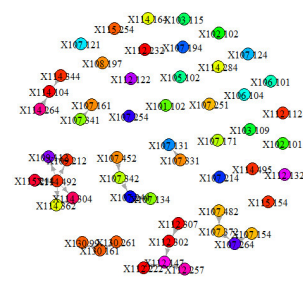

(a) Case I

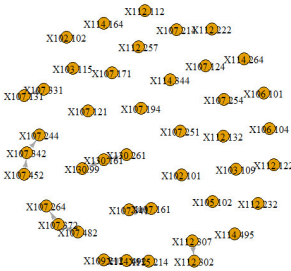

(c) Case III

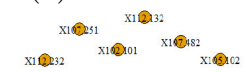

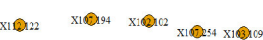

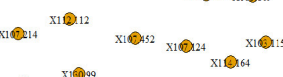

x10

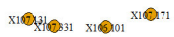

(e) Case V

x1ख15

x12:22 $\quad$ x11232

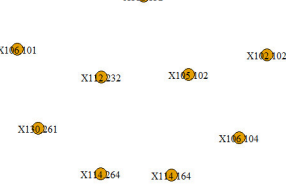

(g) Case VII

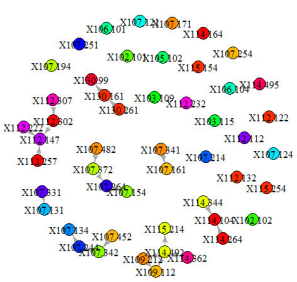

(b) Case II

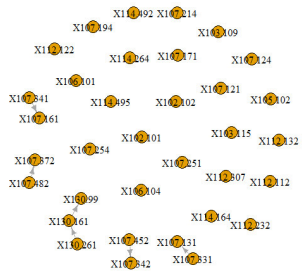

(d) Case IV

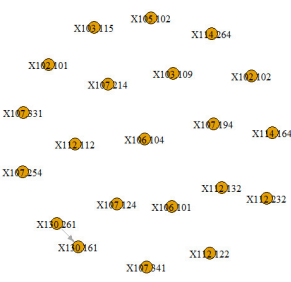

(f) Case VI

x1109

x1012

h) Case VIII

FIG. 5.2. Structure of the course network after removing courses every term

by a student in order to graduate in time. This choice of courses keeps track of his time to graduation. The dynamic course preference algorithm takes the student decision and based on the decision his courses and number of terms required to graduation is presented which enables the student to plan for extra courses in the upcoming terms and supports him in deciding on the summer term. The framework can be extended to trace the progress of the students based on the grades obtained and the number of courses chosen along with the cruciality factor to have a positive impact on the graduation rates. The difficulty analysis helps in maintaining 
TABLE 5.5

Mean Grades indicating the course difficulty

\begin{tabular}{|c|c|c|}
\hline Courses & Mean grades & SD \\
\hline X103.115 & 1.287398716 & 0.222689073 \\
\hline X103.105 & 1.394775842 & 0.276204191 \\
\hline X102.101 & 1.555222426 & 0.356798592 \\
\hline X102.102 & 1.819692206 & 0.203383015 \\
\hline X106.104 & 2.016552526 & 0.210480467 \\
\hline X114.344 & 2.164735106 & 0.51446792 \\
\hline X101.102 & 2.206264413 & 0.69981009 \\
\hline X106.101 & 2.235902851 & 0.249309948 \\
\hline X114.284 & 2.30080383 & 0.502985535 \\
\hline X115.214 & 2.328451714 & 0.595838926 \\
\hline X109.112 & 2.410101831 & 0.316662409 \\
\hline X114.104 & 2.449580312 & 0.41757027 \\
\hline X114.164 & 2.506503807 & 0.791445629 \\
\hline X114.304 & 2.524069967 & 0.82630547 \\
\hline X112.147 & 2.547242765 & 0.341405059 \\
\hline X114.384 & 2.594689678 & 0.654464115 \\
\hline X115.254 & 2.623985029 & 0.672616448 \\
\hline X115.154 & 2.634053128 & 0.324480842 \\
\hline X130.261 & 2.636714159 & 0.318058341 \\
\hline X112.232 & 2.654884638 & 0.416786992 \\
\hline X107.194 & 2.704502393 & 0.590925762 \\
\hline X115.114 & 2.716668919 & 0.314458112 \\
\hline X130.161 & 2.764785793 & 0.561615129 \\
\hline X112.112 & 2.768196564 & 0.4150545 \\
\hline X107.154 & 2.771499036 & 0.468601654 \\
\hline & \multicolumn{2}{|c}{}
\end{tabular}
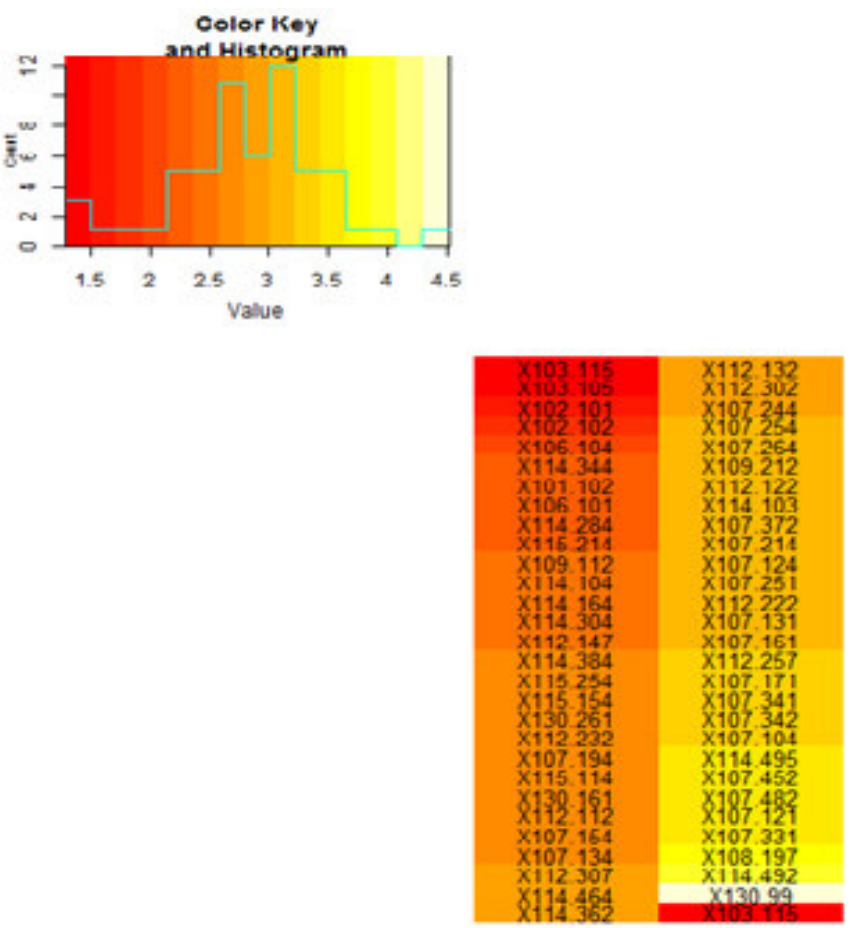

FIG. 5.3. Difficulty level of all courses in the network. 

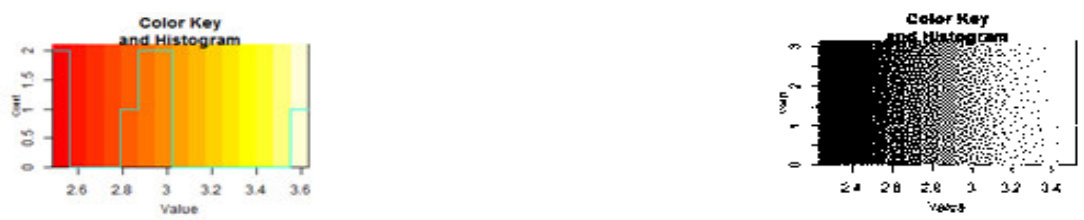

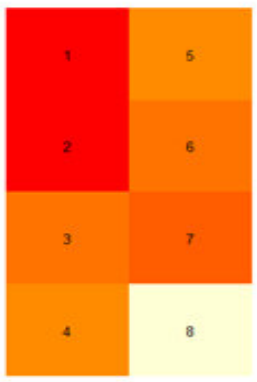

(a) Case I

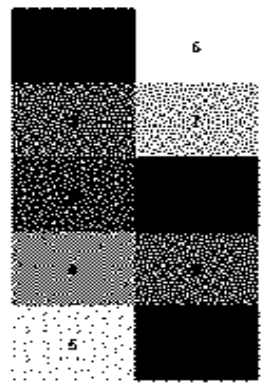

(b) Case II

FIG. 5.4. Difficulty level of terms (a) static term difficulty (b) Dynamic term difficulty

a medium hard semester for the student. The student can decide for himself the hardness of a term based on his personal conditions. The entire system is provided as software as service application in the cloud and enable student to register for course from any where irrespective of his location.

\section{REFERENCES}

[1] C. L. Sa, D. H. B. Abang Ibrahim, E. Dahliana Hossain, M. Bin Hossin, "Student performance analysis system (SPAS), Information and Communication Technology for The Muslim World (ICT4M) 2014 The 5th International Conference on, pp. 1-6, 2014.

[2] Kundariya, Daxa and Vaseem, ,A Case Study For Student Performance Analysis Based On Educational Data Mining (Edm), (2016), 10.5281/zenodo.168107.

[3] Pooja Thakar, Anil Mehta, Manisha ,Performance Analysis and Prediction in Educational Data Mining: A Research Travelogue, International Journal of Computer Applications (0975 8887) Volume 110 No. 15, January 2015

[4] Ahmed, A.B.E.D. and Elaraby, I.S.,Data Mining: A prediction for Students Performance Using Classification Method, World Journal of Computer Application and Technology, 2014 2(2), pp.43-47.

[5] Al-Radaideh, Q., Al-Shawakfa, E. and Al-NajJar, M., Mining Student Data Using Decision Trees, The 2006 International Arab Conference on Information Technology(ACIT2006),2006, Conference Proceedings.

[6] Zhu, Li, Yanli Li, and Xiang Li,Research on EarlyWarning Model of Students Academic Records Based on Association Rules, Computer Science and Information Engineering, 2009 WRI World Congress on. Vol. 4. IEEE, 2009.

[7] G. D. Kuh, J. Kinzie, J. A. Buckley, B. K. Bridges, And J. C. HayeK, What matters to student success: A review of the literature, National Postsecondary Education Cooperative, U.S. Department of Education, Commissioned Report for the National Symposium on PostsecondaryStudent Success: Spearheading a Dialog on Student Success, Tech. Rep.,2006

[8] G. D. Kuh, J. Kinzie, J. H. Schun, And E. J. Whitt,Student Success in College: Creating Conditions That Matter, San Francisco, CA: Jossey- Bass, 2010.

[9] Ahmad Slim, Jarred Kozlick, Gregory L. Heileman, Jeff Wigdahl and Chaouki T. Abdallah, Network Analysis of University Courses, International World Wide Web Conference Committee (IW3C2), WWW14 Companion, April 7-11, 2014, Seoul, Korea. ACM 978-1-4503-2745-9/14/04.

[10] Tushar Ojha, Prediction of Graduation Delay Based on Student Characteristics and Performance, University of New Mexico, UNM Digital Repository, Electrical and Computer Engineering ETDs 2017.

[11] Vincent Tinto, Dropout from higher education: A theoretical synthesis of recent research, Review of Educational Research, $1(45): 89-125,1975$.

[12] George D. Kuh, Jillian Kinzie, John H. Schuh, and Elizabeth J. Whitt. Stu- dent Success in College: Creating Conditions That Matter,Jossey-Bass, San Francisco, CA, 2010.

[13] Ahmad Slim, Jarred Kozlick, Gregory L. Heileman, and Chaouki T.Abdallah, The complexity of university curricula according to course cruciality, In Proceed ings of the 8th International Conference on Complex, Intelligent, and Software Intensive Systems, Birmingham City University, Birmingham, UK, 2014. IEEE.

[14] Ahmad Slim, Jarred Kozlick, Gregory L. Heileman, Jeo Wigdahl, and Chaouki T. Abdallah. Network analysis of university courses. In Proceedings of the 6th Annual Workshop on Simplifying Complex Networks for Practitioners, Seoul, Korea, 2014. ACM. 
[15] Jonathan Kim, The 5 Most Commonly Found Metrics for Student Success, Envisions resources, 2017.

[16] A. T. M. Shakil Ahamed, Navid Tanzeem Mahmood and Rashedur M Rahman, An intelligent system to predict academic performance based on different factors during adolescence, Journal of Information and Telecommunication,(2017) 1:2, 155-175, DOI: $10.1080 / 24751839.2017 .1323488$

[17] RaheelaAsif, Agathe Merceron, Syed AbbasAli and Najmi Ghani- Haider, Analyzing undergraduate students performance using educational data mining, Computers \& Education, Volume 113, October 2017, pp. 177-194.

[18] Ruban, L. M., McCoACh, D. B.,Gender differences in explaining grades using structural equation modelling, Review of Higher Education 284755022005.

[19] Kuncel, N. R., Crede, M., Thomas, L. L., The validity of selfreported grade point averages, class ranks, and test scores: A meta analysis and review of the literature, Review of Educational Research 75163822005.

[20] A. Stim.,Curricular Analytics in Higher Education. PhD thesis, University of New Mexico, 2016

[21] J. Wigdahl, G. L. Heileman, A. Slim, and C. T. Abdallah, Curricular efficiency: What role does it play in student success?, In 2014 ASEE Annual Conference \& Exposition, Indianapolis, Indiana, June 2014. ASEE Conferences.

[22] Daniel James Mundfrom, Estimating course difficulty, IOWA State University, Digital repository, 1991.

Edited by: Rajkumar Rajasekaran

Received: Jul 29, 2018

Accepted: Dec 3, 2018 



\title{
PARALLEL SEED SELECTION METHOD FOR OVERLAPPING COMMUNITY DETECTION IN SOCIAL NETWORK
}

\author{
BELFIN R.V.\& E. GRACE MARY KANAGA*
}

\begin{abstract}
Social network analysis is one of the key areas of research during modern times. The social network is growing with more users and the ties between them day by day. This reason brings out many research queries and new conclusions from this area. Overlapping community detection in the social network is one such research problem which has acquired interest among researchers nowadays. Earlier, the investigation was in finding out algorithms to detect communities in the network sequentially. There are many distinguished findings toward overlapping community detection. Due to the velocity of data in the current era, the available algorithms will be a bit sluggish in processing the data. The proposed algorithm uses parallel processing engine to resolve this delay problem in the current scenario. The algorithm in parallel finds out the superior seed set in the network and expands it in parallel to find out the community. The work shows amazing improvement in the runtime and also detects quality groups in the network.
\end{abstract}

Key words: Overlapping community detection, Seed selection, Graph parallel processing, seed expansion

AMS subject classifications. 65Y05, 68R10, 91D30

1. Introduction. Complex networks are commonly utilized for modeling the synergies in real-world systems in various areas, such as sociology, biology, knowledge dispersion and many different fields. One important feature of this complex network is that the nodes are tightly connected with each other in groups and in turn, the groups will be loosely connected to each other. The tightly connected groups are called as communities. The communities usually have common properties. Therefore, finding the communities from a complex network could give many insights about the network. The community detection can be done in two different ways: Firstly, the network partition can be calculated to cut the graph into partitions. Secondly, the selection of seed nodes will be done and the local communities will be centered around these seeds.

The proposed work is of the latter category where, the most important nodes in the community will be identified using a parallel superior seed set selection $(\mathrm{P} 4 \mathrm{~S})$ algorithm. The identified superior seeds will be expanded by their neighborhood till it reaches the next seed. Since the algorithm expands using the neighborhood, it will form the closely knitted group around the seed nodes.

Seed selection process is an important process in the field of network science. Usually, the measures for calculating the important nodes from the target network is named as centrality measures [24, 25]. There are many centrality measures, to name a few, Degree [31], Betweenness [34], Closeness [18], Eigen vector [35], Page Rank [32] and so on. These seed nodes play an important role in finding a good community in community detection problem and fast spreading in information diffusion application.

2. Related Works. Data nowadays is huge and need to be processed as fast as possible. There is a need of parallel algorithms to process the volume of data which comes in high velocity. Processing the data in the faster may fetch the organizations a good profit. There are lots of seed selection algorithms available for different applications. Each application might have to adopt different seed selection algorithms which match the application requirement. For example, if the application is a marketing application, the out-degree centrality or the page rank centrality may be used for the seed selection process. So, each centrality measures[9, 10, 2] will have their own limitations with various applications. The proposed method finds out a generic seed by combining various centralities.

There are some related works available in the literature. There are some diffusion models [40, 28, 21, 7] and community detection algorithms $[36,3,26,14,11]$ which will have a seed selection part in their model $[22,12,27,6]$. Some of the algorithms selects the seeds in random and optimize its result at the end of the process [15]. There is a excellent study on seed nodes in [13]. Evolutionary algorithms are used to find out the seed nodes in some cases [34].

*Department of Computer Science and Engineering, Karunya institute of technology and sciences, Coimbatore, Tamilnadu, India. (belfin@karunya.edu, grace@karunya.edu). 
There are several graph computations algorithm available for large-scale graphs, various parallel modules have been developed, e.g., Pregel [20], GraphLab [19] Giraph++ [8], GraphX [37], GRACE [30], GPS [23] and Blogel [38], based on MapReduce [5] and BSP (Bulk Synchronous Parallel) models [29].

The proposed algorithm uses GraphX to process the graph in parallel. Our previous work Superior seed set selection algorithm (4S) [1] was extended to parallel processing in this article. The experiment result proves that the proposed parallel algorithm can find out the good clusters fastest then the available algorithms.

3. Problem Statement. Some of the seed selection algorithms which are already available are using random nodes for selecting the seed node. The selection of a seed node or a seed set is really important in the algorithms which use seeds to find out communities. Since, the community detection algorithm needs to work in unsupervised way the seeds should be excellent to obtain a perfect local community. The selection of excellent seeds will reduce the number iterations in the community detection algorithm.

Nowadays the data need to be processed is large because of the growing use of internet and social media. Reducing iterations will not be enough to make the algorithm work faster. There is a need of parallel algorithms which can process the data in a synchronized manner. Parallel processing algorithms are modern nowadays. The uses of these kinds of algorithms will be fast and effective.

4. Problem Formulation. Assuming an undirected graph $G=(V, E)$. The nodes of the graph $G$ be $n=|V|$ nodes and the edges of the graph $G$ be $m=|E|$. The overlapping community detection algorithm is used to determine the community $C=C_{1}, \ldots, C_{x}$ of all the nodes of $G$. $|C|$ be the number of communities identified. In the proposed work, $|C|$ is the number of seed node selected from parallel superior seed set selection algorithm.

Various centrality measures $\mu_{i}$ for a node $i$ can be calculated which will be the importance measure of node $i$. Let us assume the graph to be undirected. The traditional methods uses any one of the centrality measures with respect to the use case, to define its seed set $S(G)=s_{i}, s_{j}, \ldots, s_{\kappa}$ where $s_{i}, s_{j}, \ldots, s_{\kappa} \in V$. Some models pick seed set randomly and gets the parameter $\kappa$ as input, to decide the number of seeds in the set. The difficulty in the conventional methods is that, the seed set need to be adjusted according to the circumstances of the problem. The proposed parallel, unified model determines the superior seed set $S(G)$ from the centrality measures collectively. This article introduces a threshold value $\tau$, which limits the number of seed nodes selected for the $S(G)$.

A community may be basically described as a collection of nodes that may share common features, or engage in similar roles in the network. Also, it is tightly knitted groups with a high density of inter community ties and a low density of intra-community ties. The proposed algorithm produces results that are composed of one of two types of assignments, crisp assignment of nodes or fuzzy assignment of nodes. With crisp assignment, the relationship between a node and a cluster is binary. That is, a node $i$ either belongs to community $C$ or does not. With fuzzy assignment, each node is associated with two or more communities. Throughout the article, the terms set, cluster, and community are used interchangeably.

5. Proposed work. The main idea of this work is to use the parallel processing architecture to find out the best seeds and subsequently the best seeds from $\mathrm{P} 4 \mathrm{~S}$ algorithm will be used to find out the communities hidden in the network. The work has been done with GraphX a module in spark parallel processing engine. The algorithms for the Parallel seed selection and parallel community detection has been explained below in the following sections. The parallel community detection framework is depicted in the Fig 5.1. The input of the algorithm will be the unlabeled network, for example, social graph or collaboration networks or the network of web pages. The output of the work will be the expanded communities from the selected seeds. The goodness of the communities is tested by comparing the inter-density and intra-density of the communities along with the graph density. The algorithm for the P4S has been given in the Algorithm 1. Algorithm 2 shows the parallel seed set expansion algorithm which finds out the communities.

GraphX is the Apache Spark ingredient for graph-parallel calculations, developed upon a division of mathematics called graph theory. It is a distributed graph processing framework that lies on top of the Spark core. GraphX inherits the Spark RDD with a Resilient Distributed Property Graph. The property graph is a directed multi-graph. The multiple edges it has will be parallel. The parallel edges permit multiple relationships between the same vertices. The Fig. 5.2 shows the architecture of the parallel $4 \mathrm{~S}$ and parallel community detection implemented in the spark. 


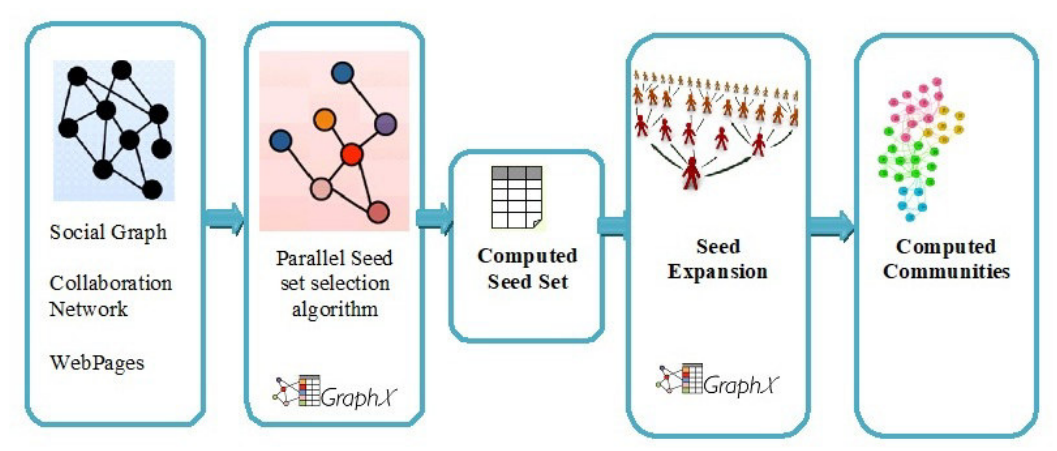

FIG. 5.1. Parallel community detection framework
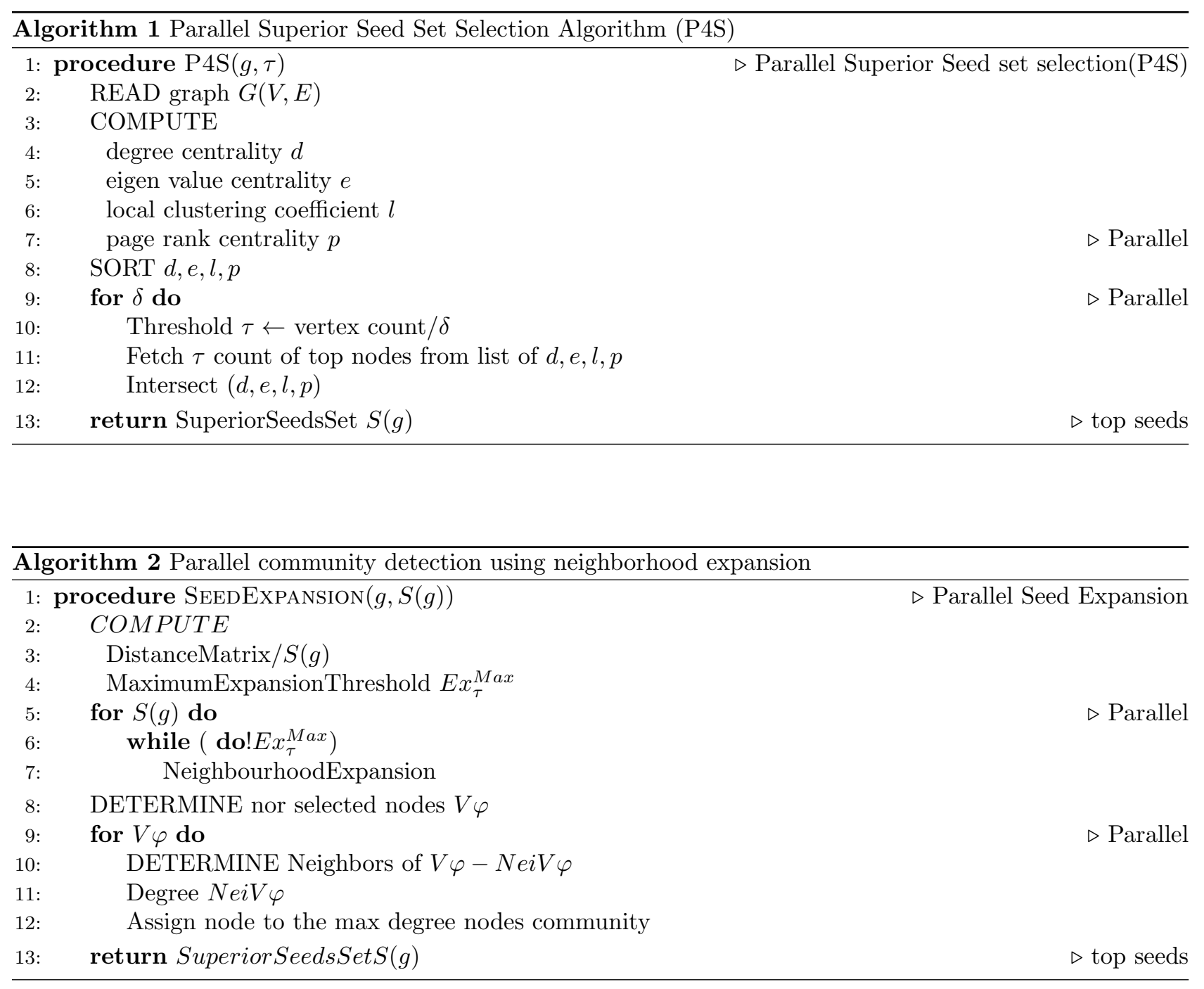


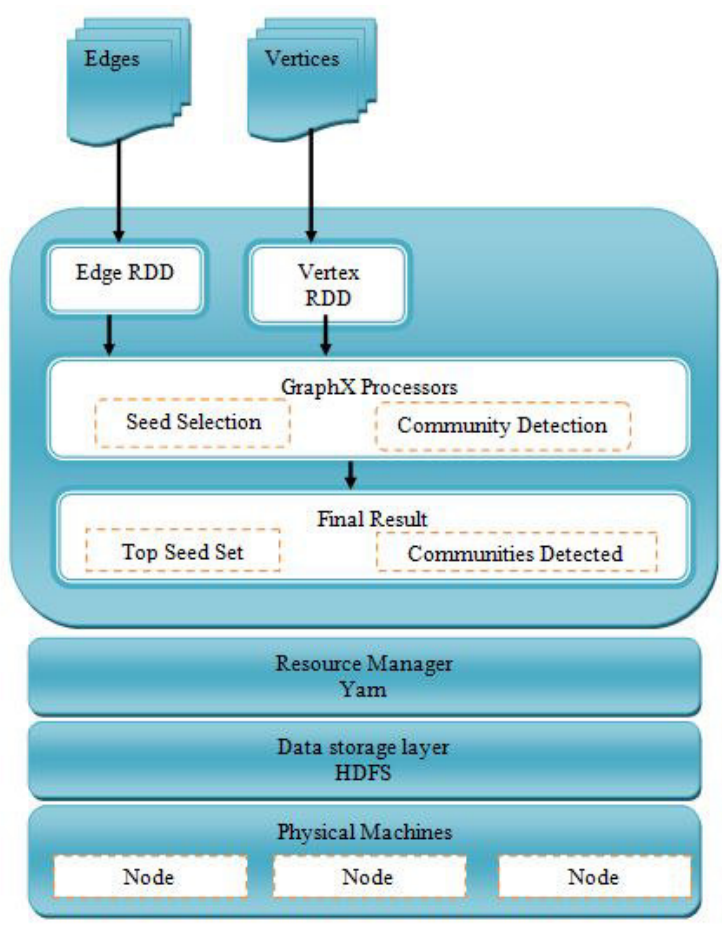

FIG. 5.2. Implementation of Parallel $4 S$ and Parallel community detection algorithm in Spark architecture

The GraphX processors will be used to compute the superior seed set and the seed expansion of the node to form community in parallel. Three node clusters was used for computing the communities in parallel.

5.1. Parallel Superior Seed Set Selection (P4S). Parallel superior seed set selection algorithm extracts the very important nodes in the input graph. The input data will be stored in the distributed file system for further processing. Centrality measures are used to coin out the important nodes in the network. Each centrality measure will have its own importance and use cases to work on. Combining these centrality measures can identify good seeds across all the centrality measures. For the experiment done for this work Page rank, Degree, Eigen value, Local clustering coefficient centralities was used. The centralities measure calculation will automatically run in parallel when GraphX is used. Sorting according to the centrality ranks will be done for all centrality measures used. A threshold value will be used to split the top ranks for all measures. Finally, set intersection of the top nodes from each centrality measure will be done to get the superior seed set. Fig. 5.3 depicts the process of finding the superior seed set.

5.2. Parallel Community Detection. Community detection from seed by expanding greedily through neighbors is a classical process. The proposed work is the modified version of the seed expansion in a parallel way. Since we have multiple seeds the algorithm will expand in parallel from the seeds till it reaches the next seed and stops expanding. The distance between the seeds will be calculated by the distance matrix. The $E x_{\tau}^{M a x}$ defined in the Algorithm 2 is the maximum expansion limit. The nodes which are not selected in the first iteration will be picked and added to its neighbors community. In case of more than one neighbor the highest degree node will add the ungrouped node to its community.

6. Experiment. The real-world datasets used for the experiments are from [17]. All the datasets are connected, undirected graphs. The datasets are from various categories like collaboration networks and product networks. The detailed information about the datasets is given in the Table 6.1.

Collaboration networks. In a collaboration network, vertices denote authors, and edges denote coauthorship. If authors $u$ and $v$ are co-authors, they will be connected by an edge. So, if an article is written 


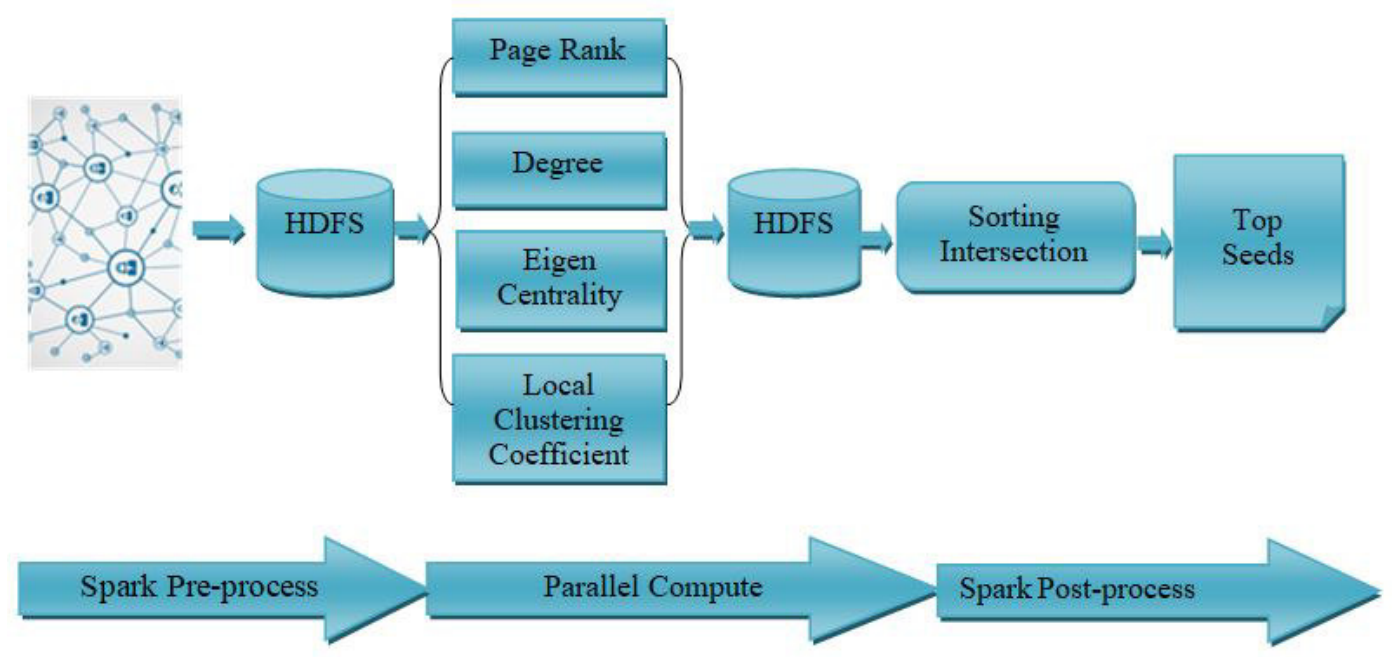

FIG. 5.3. Parallel $4 S$ algorithm for finding top seed set

TABLE 6.1

Summary of real-world networks used

\begin{tabular}{|l|l|l|l|l|l|l|}
\hline Graph & $\begin{array}{l}\text { No. of } \\
\text { Vertices }\end{array}$ & $\begin{array}{l}\text { No. of } \\
\text { edges }\end{array}$ & $\begin{array}{l}\text { Max. } \\
\text { Deg }\end{array}$ & $\begin{array}{l}\text { Avg. } \\
\text { Deg }\end{array}$ & Avg. CC & $\begin{array}{l}\text { Ground- } \\
\text { truth }\end{array}$ \\
\hline HepPh & 11,204 & 117,619 & 491 & 21.00 & 0.6216 & N/A \\
\hline AstroPh & 17,903 & 196,972 & 504 & 22.00 & 0.6328 & N/A \\
\hline CondMat & 21,363 & 91,286 & 279 & 8.50 & 0.6417 & N/A \\
\hline DBLP & 317,080 & $1,049,866$ & 343 & 6.60 & 0.6324 & Yes \\
\hline Amazon & 334,863 & 925,872 & 549 & 5.50 & 0.3967 & Yes \\
\hline Orkut & 731,332 & $21,992,171$ & 6933 & 60.10 & 0.2468 & Yes \\
\hline
\end{tabular}

by $\mathrm{n}$ authors, then their relationship will be represented as a clique in the network. HepPh, AstroPh, and CondMat networks are formed based on the journal submitted to High Energy Physics (Phenomenology) group, Astrophysics group, and Condensed Matter Physics group under the arXiv e-print service, respectively. The DBLP network is formed based on the DBLP computer science bibliography website.

Product network. In the Amazon product network, vertices denote products and edges denote copurchasing information. If products $u$ and $v$ are frequently co-purchased, then there will be an undirected edge between them.

Social networks. In a social network, vertices denote users of the social network and the edges denote social communications between them. Users can construct a friendship relationship with each other in this web application.

\section{Community Evaluation.}

7.1. Intra-Density, Inter-Density and Graph Density. The cohesiveness of the edges in a graph G can be readily attained by calculating the graph density $\rho$.

$$
\rho=\frac{|E|}{n(n-1) / 2}
$$

where $n$ is the count of nodes in the network and $n(n-1) / 2$ is the maximum possible edges and $|E|$ is the number of edges in graph $G$. 
Consider $c$ is a community in the given network $\mathrm{G}$ where $|G|=n$ and $|c|=n c$. Internal edges are edges which have it both sides situated inside the community $c$ and the external edges of community $c$ refer to the edges which connect a vertex in $c$ to the rest of the graph. The internal degree of vertex $v$ in community $c$ is denoted by $k_{v}^{i n t}$ is the number of edges connecting $v$ to other vertices in $c$ and the external degree of $v$ denoted as $k_{v}^{e x t}$ is the number of edges connecting $v$ to the rest of the graph. The intra cluster density $\delta^{i}(c)$ of a community $c$ is the ratio between the number of internal edges of $c$ and the number of all possible internal edges:

$$
\delta^{i}(C)=\frac{\left|E^{i}\right|}{n_{c}\left(n_{c}-1\right) / 2}
$$

where $\left|E^{i}\right|$ is the count of internal edges in the community.

Similarly, the inter-cluster density $\delta^{e}(c)$ is the ratio between the number of inter-cluster edges of $c$ and the number of all possible inter-cluster edges:

$$
\delta^{e}(C)=\frac{\left|E^{e}\right|}{n_{c}\left(n-n_{c}\right)}
$$

where $\left|E^{e}\right|$ is the count of inter community edges in the community.

The proposed parallel seed set selection algorithm has been implemented in the real-world datasets and has given a better result. The goodness of the community has been tested with the inter-cluster and intra-cluster density. Usually For overlapping communities, internal and external metric values will be used. Because, combination metrics and modularity scores will result in confusing values that should be inconsistent[15]. The goodness of the community using graph density $\rho$, intra-cluster density $\delta^{i}(c)$ and inter cluster density $\delta^{e}(c)$ can be given as:

$$
\delta^{i}(C)>\rho>\delta^{e}(C)
$$

The comparison of inter-cluster density, intra-cluster density and graph density of the real datasets are plotted in the result and discussion section. The experiment was done for the seed sizes $71,84,114,128,152$, 172, 207, 240 and 270.

7.2. Clustering Coefficient. A clustering coefficient is a measure of the degree to which nodes in a graph are inclined to tie together. Research results suggests that in most real-world networks, and in some social networks, nodes are likely to create tightly connected groups characterized by a pretty high density of ties; this likelihood will greater than the average probability of a connection randomly established between two nodes [33].

The local clustering coefficient $l_{i}$ for a vertex $i$ is then given by the ratio of links between the nodes within its neighbors divided by the number of edges possible between them. The local clustering coefficient for an undirected graph is given as:

$$
C_{i}=\frac{2\left|e_{j, n e i}: v_{j}, v_{n e i} \in N_{i}, e_{j, n e i} \in E\right|}{n e i_{i}\left(n e i_{i}-1\right) / 2}
$$

where vertex $v_{j}$, has $n e i_{i}$ neighbors, $\frac{n e i_{i}\left(n e i_{i}-1\right)}{2}$ edges will be the maximum possible edges exist among the vertices within the neighborhood.

8. Result and discussion. The problem of community assessment is still an open and difficult problem in spite of huge sum of work addressing this topic [13]. The experiment done on the real-world datasets are given on the summary Table 6.1. The values of inter-density, intra-density and graph density are compared to find the goodness of the cluster. The result from the six real-world datasets specified in Table 6.1 has been plotted in the Fig 8.1. Every dataset used for the experiment passes the test and it goes hand in hand with the Eq. (7.4). In all cases the intra-cluster density $\delta^{i}(c)$ from Eq. (7.2) of all the communities identified is greater than the graph density $\rho$ and the inter-cluster density $\delta^{e}(c)$ from Eq. 7.3 of nodes after finding communities is lesser then the graph density $\rho$ from Eq. 7.1. 

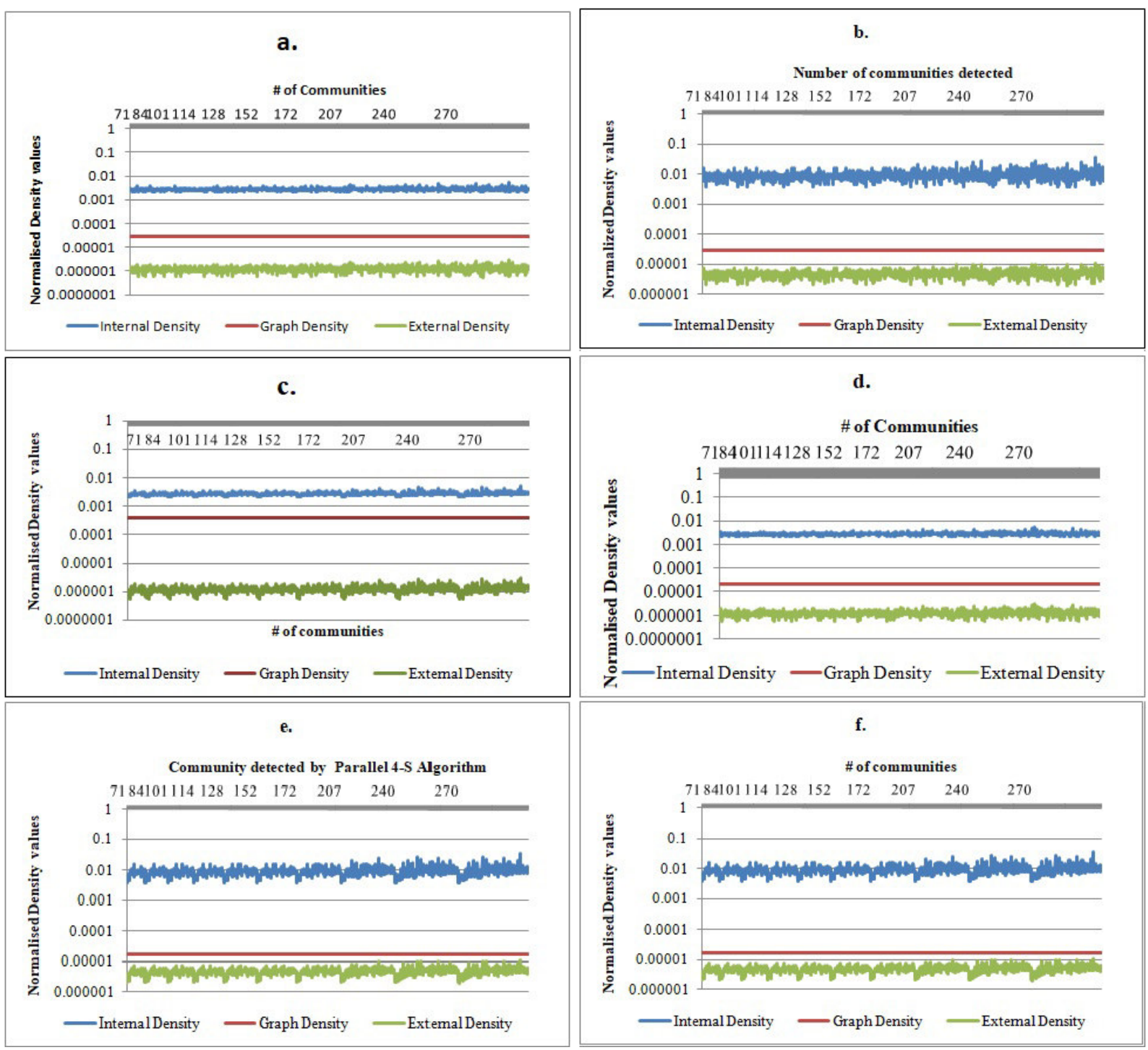

FIG. 8.1. Data comparison between the inter-cluster density, intra-cluster density and graph density: (a) Amazon dataset (b) AstroPh (c) CondMat (d) DBLP (e) HepPh (f) Orkut

The clustering coefficient can be a measure to find out the closely connected groups. The clustering coefficient equation is given the Eq. 7.5. The comparisons of clustering coefficient with the number of communities are given in the Fig. 8.2. The clustering coefficient of two datasets namely Amazon Fig. 8.2a and AstroPh Fig. $8.2 \mathrm{~b}$ are shown. The result shows an increasing trend in the clustering coefficient as the number of seeds increases. This shows that the larger the seed set higher the density of the community. Fig. 8.3 shows the comparison of clustering coefficient and number of nodes in each community. The result of comparison of average clustering coefficient of the entire network and the average community clustering coefficient is compared in the Fig. 8.4a and the comparison of average degree of the entire graph and the average degree of all the communities detected are shown as a bar plot in Fig. 8.4b. The plots clearly show that the density of the sub graphs increases after the community detection.

The running time factor is one of the important problems in community detection algorithms. When the input graph becomes larger the operations in graph will become complex and operations on it will be costly. The running time of the algorithm was compared with five important algorithms from the literature shown in 


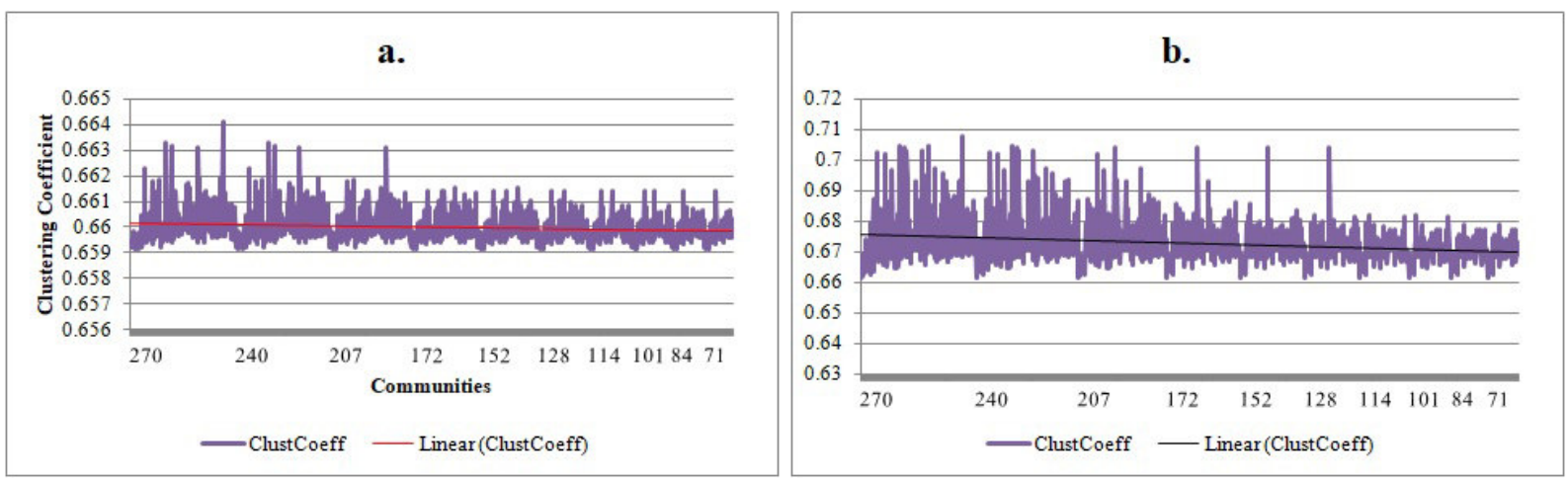

FIG. 8.2. Number of communities VS Clustering Coefficient (a) Amazon (b)AstroPh
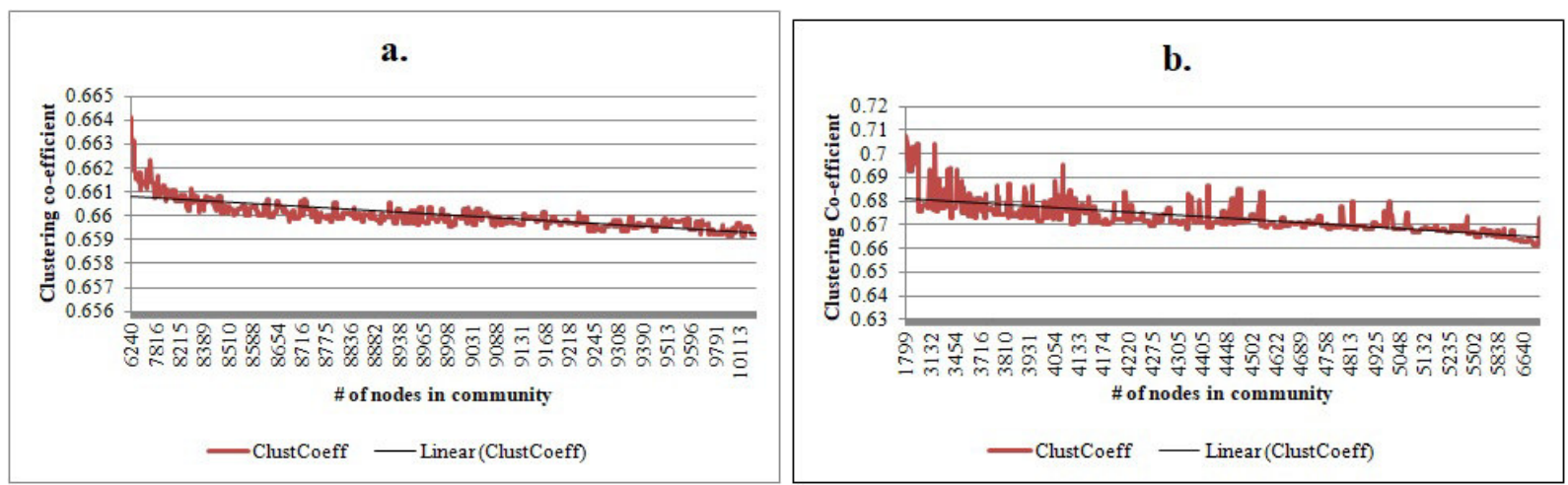

FIG. 8.3. Number of nodes in the community VS Clustering Coefficient (a) Amazon (b)AstroPh

the Table 8.1. OSLOM [16], DEMON [4], Big Clam [39], nise-sph-fppr and nise-grc-fppr from [35].

As it can be clearly seen in the Table 8.1 the proposed P4S algorithm with seed expansion algorithm works faster than the other algorithms compared. Since it is parallel execution, the algorithm finishes its execution faster than the other algorithms compared.

The details in the Table 8.2 depict the coverage (\%) of each algorithm. In this case the proposed algorithm doesnt gives $100 \%$ as nise-sph-fprr and nise-grc-fppr [35]. When the number of communities is considered the proposed $\mathrm{P} 4 \mathrm{~S}$ method gives better result than the algorithms compared.

9. Conclusion. The proposed $\mathrm{P} 4 \mathrm{~S}$ method has been implemented and tested in this article. The work gives encouraging result and it could produce better communities in less time. The P4S selects very good seeds and because of the seeds he communities can be expanded from the seeds easily. Since the seeds have a good density of nodes around it, the expansion will be faster when the process starts and it drags a little to complete. The generated communities from this method prove the seeds selected are excellent. The goodness metric of the communities selected has been tested with the density metrics. The density test proves that the communities generated are also good. The communities detected has also been tested with the clustering coefficient and proved to be good after the detection of community. The trend line shows that larger the seed set higher the density will be. The runtime of the algorithm was also calculated and it shows a very good improvement. Finally, the coverage of the algorithm was tested and the P4S covers more than $95 \%$ in most of the iterations. When considering the number of communities with the coverage $\%$ the result $\mathrm{P} 4 \mathrm{~S}$ gave is better. The algorithm still needs many improvements with respect to the centrality measures selection, range of threshold values and seed expansion approach. 

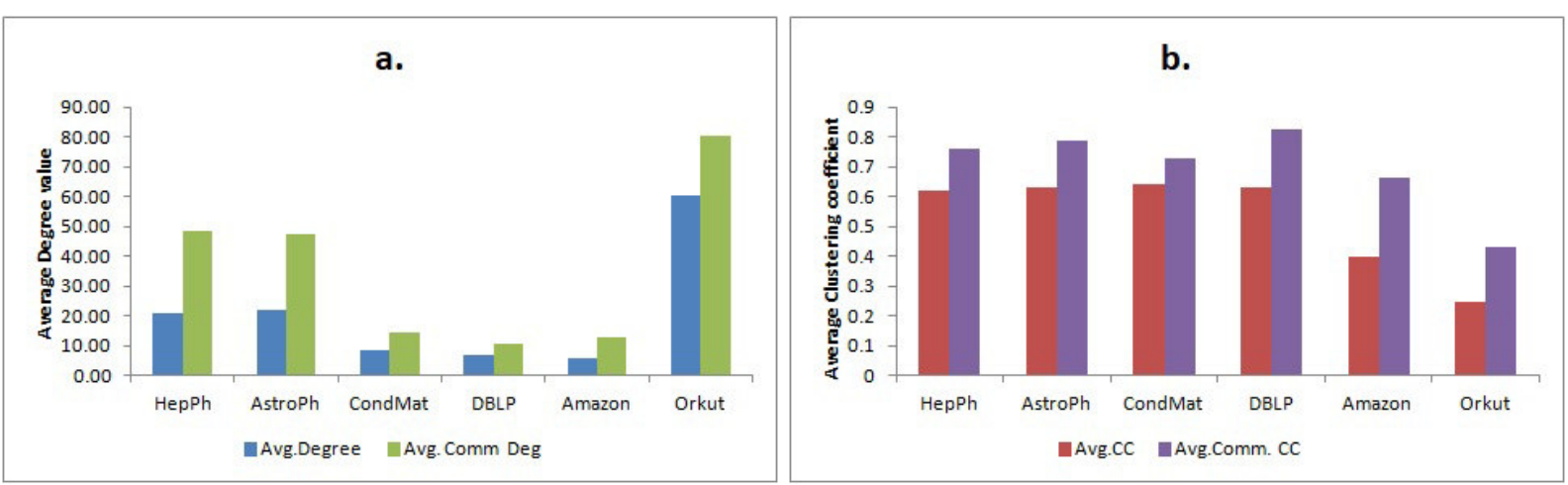

FIG. 8.4. (a) Comparison of average clustering coefficient of nodes before and after community detection. (b) Comparison of average degree of nodes before and after community detection.

TABLE 8.1

Running times of different methods on our test networks in (Minutes)

\begin{tabular}{|l|l|l|l|l|l|l|}
\hline Graph & oslom & demon & bigclam & nise-sph-fppr & nise-grc-fppr & P4S \\
\hline HepPh & 19.26 & 0.45 & 11.383 & 0.36 & 2.8 & 0.3 \\
\hline AstroPh & 38.05 & 0.7 & 48.016 & 0.6 & 2.43 & 0.483 \\
\hline CondMat & 20.65 & 0.83 & 7.35 & 0.6 & 1.23 & 0.45 \\
\hline DBLP & 350 & 233 & 433 & 18.33 & 29.733 & 12.6 \\
\hline Amazon & 175 & 115 & 85 & 37.6 & 42.716 & 20.3 \\
\hline Orkut & N/A & N/A & 4199 & 43.916 & 236 & 31.83 \\
\hline
\end{tabular}

TABLE 8.2

Returned number of communities and graph coverage of each algorithm

\begin{tabular}{|l|l|l|l|l|l|l|l|}
\hline Graph & & oslom & demon & bigclam & nise-sph-fppr & nise-grc-fppr & P4S \\
\hline \multirow{2}{*}{ HepPh } & Coverage (\%) & 100 & 88.3 & 84.37 & 100 & 100 & 94.83 \\
& no.of.clusters & 608 & 5,147 & 100 & 99 & 90 & 270 \\
\hline \multirow{2}{*}{ AstroPh } & Coverage (\%) & 100 & 94.15 & 91.11 & 100 & 100 & 95.4 \\
& no.of.clusters & 1,241 & 8,259 & 200 & 212 & 246 & 270 \\
\hline \multirow{2}{*}{ CondMat } & Coverage (\%) & 100 & 91.16 & 99.96 & 100 & 100 & 98.5 \\
& no.of.clusters & 1,534 & 10,474 & 200 & 201 & 249 & 270 \\
\hline \multirow{2}{*}{ DBLP } & Coverage (\%) & 100 & 84.89 & 100 & 100 & 100 & 97.4 \\
& no.of.clusters & 17,519 & 174,560 & 25,000 & 26,503 & 18,477 & 270 \\
\hline \multirow{2}{*}{ Amazon } & Coverage (\%) & 100 & 79.16 & 100 & 100 & 100 & 96.71 \\
& no.of.clusters & 17,082 & 105,685 & 25,000 & 27,763 & 20,036 & 270 \\
\hline \multirow{2}{*}{ Orkut } & Coverage (\%) & N/A & N/A & 82.13 & 99.99 & 99.99 & 98.5 \\
& no.of.clusters & N/A & N/A & 25,000 & 25,204 & 32,622 & 270 \\
\hline
\end{tabular}




\section{REFERENCES}

[1] R. Belfin, G. E., and P. Bródka. Overlapping community detection using superior seed set selection in social networks. Computers and Electrical Engineering, 2018.

[2] P. Bonacich. Power and Centrality: A Family of Measures. American Journal of Sociology, 1987.

[3] T. Chakraborty, S. Srinivasan, N. Ganguly, A. Mukherjee, and S. Bhowmick. Permanence and Community Structure in Complex Networks. 11(2), 2016.

[4] M. Coscia, G. Rossetti, F. Giannotti, and D. Pedreschi. DEMON: a Local-First Discovery Method for Overlapping Communities. 2012.

[5] J. Dean and S. Ghemawat. MapReduce: Simplified Data Processing on Large Clusters. Communications of the ACM, 2008.

[6] S. Dhamal, P. K. J., and Y. Narahari. Information Diffusion in Social Networks in Two Phases. IEEE Transactions on Network Science and Engineering, 4697(c):1-1, 2016

[7] F. Erlandsson, P. Brdóka, and A. Borg. Seed selection for information cascade in multilayer networks. Studies in Computational Intelligence, 689:426-436, 2018.

[8] W. Fan, J. Xu, X. Luo, Y. Wu, W. Yu, and R. Xu. GRAPE: Conducting Parallel Graph Computations without Developing Parallel Algorithms. pages 30-41.

[9] L. C. Freeman. Centrality in Social Networks. Social Networks, 1978.

[10] L. C. Freeman. Centrality in social networks conceptual clarification. Social Networks, 1978.

[11] J. Han, W. Li, Z. Su, L. Zhao, and W. Deng. Community detection by label propagation with compression of flow. 2016.

[12] L. G. S. Jeub, M. W. Mahoney, P. J. Mucha, and M. A. Porter. A Local Perspective on Community Structure in Multilayer Networks. volume 5, pages 144-163, 2015.

[13] R. Kanawati. Seed-Centric Approaches for Community Seed-Centric Algorithms : A Classification Study. pages 197-208, 2014.

[14] R. Kanawati. YASCA: An Ensemble-Based Approach for Community Detection in Complex Networks. Algorithmica, 76(4):657-666, 2014.

[15] A. Lancichinetti and S. Fortunato. Community detection algorithms: A comparative analysis. Physical Review E - Statistical, Nonlinear, and Soft Matter Physics, 80(5):1-11, 2009.

[16] A. Lancichinetti, F. Radicchi, J. J. Ramasco, and S. Fortunato. Finding statistically significant communities in networks. PLoS ONE, 6(4), 2011.

[17] S. Large and N. Dataset. Stanford Large Network Dataset Collection, 2013.

[18] Y. Li, Y. Wang, J. Chen, L. Jiao, and R. Shang. Overlapping community detection through an improved multi-objective quantum-behaved particle swarm optimization. Journal of Heuristics, 21(4):549-575, 2015.

[19] Y. Low, J. Gonzalez, A. Kyrola, D. Bickson, C. Guestrin, and J. M. Hellerstein. GraphLab: A New Framework for Parallel Machine Learning. The 26th Conference on Uncertainty in Artificial Intelligence (UAI 2010), 2010.

[20] G. Malewicz, M. H. Austern, A. J. Bik, J. C. Dehnert, I. Horn, N. Leiser, and G. Czajkowski. Pregel: a system for large-scale graph processing. Proceedings of the 2010 international conference on Management of data - SIGMOD '10, 2010.

[21] R. Michalski, T. Kajdanowicz, P. Bródka, and P. Kazienko. Seed selection for spread of influence in social networks: Temporal vs. static approach. New Generation Computing, 32(3-4):213-235, 2014.

[22] C. H. Mu, J. Xie, Y. Liu, F. Chen, Y. Liu, and L. C. Jiao. Memetic algorithm with simulated annealing strategy and tightness greedy optimization for community detection in networks. Applied Soft Computing Journal, 34:485-501, 2015.

[23] S. Salihoglu and J. Widom. GPS : A Graph Processing System. SSDBM Proceedings of the 25th International Conference on Scientific and Statistical Database Management, 2013.

[24] A. Sol, S. G. e-Ribalta, Manlio De Domenico, and A. A. Omez. Random walk centrality in interconnected multilayer networks. Physica D: Nonlinear Phenomena, 323-324:73-79, 2016.

[25] L. Solá, M. Romance, R. Criado, J. Flores, A. García del Amo, and S. Boccaletti. Eigenvector centrality of nodes in multiplex networks. Chaos, 23(3):1-11, 2013.

[26] G. Song, Y. Li, X. Chen, X. He, and J. Tang. Influential Node Tracking on Dynamic Social Network: An Interchange Greedy Approach. IEEE Transactions on Knowledge and Data Engineering, 29(2):359-372, 2017.

[27] A. Srivastava, C. Chelmis, and V. Prasanna. Social influence computation and maximization in signed networks with competing cascades. Proceedings of the 2015 IEEE/ACM International Conference on Advances in Social Networks Analysis and Mining, ASONAM 2015, pages 41-48, 2015.

[28] V. Tejaswi, P. V. Bindu, and P. S. Thilagam. Diffusion models and approaches for influence maximization in social networks. In 2016 International Conference on Advances in Computing, Communications and Informatics, ICACCI 2016, pages 1345-1351, 2016.

[29] L. G. . V. . Valiant. A Bridging Model for Parallel Computation. Communications of the ACM, 1990.

[30] G. Wang, W. Xie, A. Demers, and J. Gehrke. Asynchronous Large-Scale Graph Processing Made Easy. Cidr, 2013.

[31] M. Wang, C. Wang, J. X. Yu, and J. Zhang. Community Detection in Social Networks : An In-depth Benchmarking Study with a Procedure-Oriented Framework. Proceedings of the VLDB Endowment, pages 998-1009, 2015.

[32] Y. Wang, B. Zhang, A. V. Vasilakos, and J. Ma. PRDiscount: A Heuristic scheme of initial seeds selection for diffusion maximization in social networks. In Lecture Notes in Computer Science (including subseries Lecture Notes in Artificial Intelligence and Lecture Notes in Bioinformatics), volume 8588 LNCS, pages 149-161, 2014.

[33] D. J. Watts and S. H. Strogatz. Collective dynamics of small-world' networks. Nature, 393(6684):440-442, 1998.

[34] M. Weskida. Evolutionary Algorithm for Seed Selection in Social Influence Process. pages 1189-1196, 2016.

[35] J. J. Whang, D. F. Gleich, and I. S. Dhillon. Overlapping Community Detection Using Neighborhood-Inflated Seed Expansion. IEEE Transactions on Knowledge and Data Engineering, 28(5):1272-1284, 2016. 
[36] J. J. Whang, P. Rai, and I. S. Dhillon. Stochastic blockmodel with cluster overlap, relevance selection, and similarity-based smoothing. Proceedings - IEEE International Conference on Data Mining, ICDM, pages 817-826, 2013.

[37] R. S. Xin, J. E. Gonzalez, M. J. Franklin, and I. Stoica. GraphX. In First International Workshop on Graph Data Management Experiences and Systems - GRADES '13, pages 1-6, 2013.

[38] D. Yan, J. Cheng, Y. Lu, and W. Ng. Blogel - a block-centric framework for distributed computation on real-world graphs. Proceedings of the VLDB Endowment, 7(14):1981-1992, 2014.

[39] J. Yang and J. Leskovec. Overlapping community detection at scale: A Nonnegative Matrix Factorization Approach. Sixth ACM international conference on Web search and data mining, page 587, 2013.

[40] J. U. N. Zhang, F. Xia, S. Member, and Z. Ning. A Hybrid Mechanism for Innovation Diffusion in Social Networks. $4,2016$.

Edited by: Rajkumar Rajasekaran

Received: Jul 30, 2018

Accepted: Dec 14, 2018 



\title{
ENERGY-EFFICIENT REAL-TIME SCHEDULING ALGORITHM FOR FAULT-TOLERANT AUTONOMOUS SYSTEMS*
}

\author{
HUSSEIN EL GHOR $\dagger$ JULIA HAGE $\ddagger$ NIZAR HAMADEH§ AND RAFIC HAGE CHEHADE
}

\begin{abstract}
For the past decades, we have experienced an aggressive technology scaling due to the tremendous advancements of Integrated Circuit technology. As massive integration continues, the power consumption of the IC chips exponentially increases which further degraded the system reliability. This in turn poses significant challenges to the design of real-time autonomous systems. In this paper, we target the problem of designing advanced real-time scheduling algorithms that are subject to timing, energy consumption and fault-tolerant design constraints. To this end, we first investigated the problem of developing scheduling techniques for uniprocessor real-time systems that minimizes energy consumption while still tolerating up to $k$ transient faults to preserve the system's reliability. Two scheduling algorithms are proposed: the first scheduler is an extension of an optimal faultfree energy-efficient scheduling algorithm, named ES-DVFS. The second algorithm aims to enhance the energy saving by reserving adequate slack time for recovery when faults strike. We derive a necessary and sufficient condition that must be efficiently checked for the time and energy feasibility of aperiodic jobs in the presence of failures. Later, we formally prove that the proposed algorithm is optimal for a $k$-fault-tolerant model. Our simulation results demonstrate that the proposed schedulers can efficiently improve energy savings when compared with previous works.
\end{abstract}

Key words: Real-time systems, real-time scheduling, fault tolerance, energy consumption, processor demand, ES-DVFS scheduler

AMS subject classifications. $68 \mathrm{M} 15,94 \mathrm{C} 12$

1. Introduction. Autonomous systems are becoming increasingly important in our lives. In these autonomous devices, the management of energy is a crucial issue. They are more and more varied and appear in extremely diverse sectors such as transport (avionics, cars, buses, ..), multimedia, mobile phones, game consoles, etc. A large part of autonomous systems have needs for autonomy and limitations of space (small size) and energy (limited consumption). As a result, the major technological and scientific challenge is to build systems of trust from the point of view of the functionalities provided and the rendered quality of service. Its more about designing these systems at an acceptable cost.

For the past several decades, we have experienced tremendous growth of real-time systems and applications largely due to the remarkable advancements of IC technology. However, as transistor scaling and massive integration continue, the dramatically increased power/energy consumption and degraded reliability of IC chips have posed signficant challenges to the design of real-time embedded systems [1]. Hence, it is imperative to propose efficient and effective power/energy management techniques for real-time systems while still guaranteeing the timing constraints. For the past years, extensive real-time energy-efficient scheduling algorithms have been proposed to minimize the processor energy consumption for embedded systems [2], [3].

Such a problem is usually treated by Dynamic Voltage and Frequency Scaling (DVFS) methods that affect the processor speed, which directly affects the energy consumption of the system. The energy-efficient scheduling of real-time jobs on a DVFS processor has been extensively studied in the previous decade [4], [5], [6].

At the same time, it is observed that as autonomous real-time systems become more and more complex, the required level of reliability for such systems appears to be another open problem. Such comples systems are usually situated at harsh, remote or inaccessible locations. Consequently, it is often difficult and sometimes even impossible to repair and to perform maintenance. This necessitates the use of fault-tolerant techniques. Fault-tolerant computing stands for the reliable (correct) execution of system software and user programs in the presence of failures [7]. Nowadays, the impacts of system failures become more and more substantial, ranging from personal inconvenience, disruption of our daily lives, to some catastrophic consequences such as huge financial loss. Conceivably, guaranteeing the reliability of computing systems has also been raised

\footnotetext{
*This work was supported by the Laboratory of Embedded and Networked Systems at the Lebanese University.

${ }^{\dagger}$ LENS Laboratory, Faculty of Technology, Lebanese University, B.P. 813 Saida, LEBANON. (husseinelghor@ul.edu. 1b).

${ }^{\ddagger}$ Faculty of Technology, Lebanese University, B.P. 813 Saida, LEBANON.

$\S$ LENS Laboratory, Faculty of Technology, Lebanese University, B.P. 813 Saida, LEBANON (nizar.hemadeh@ul.edu.1b).

"LENS Laboratory, Faculty of Technology, Lebanese University, B.P. 813 Saida, LEBANON (rhagechehade@ul.edu. lb).
} 
to be a first-class design concern. Recent studies indicate that the emerging energy-efficient design techniques further increase the susceptibility of VLSI circuits to transient faults [4]. Left unchecked, the high power/energy consumption and deteriorating reliability of IC chips will handicap the availability of future generations of realtime computing systems. Hence, faults have to be detected and convenient recovery methods must be performed within the timing constraints.

Processor faults can be mainly seperated into two categories: transient and permanent faults [8]. Transient faults are temporary malfunctioning of the computing unit or any other associated components caused by factors such as electromagnetic interference and cosmic ray radiations, which causes incorrect results to be computed. On the contrary, a permanent or hard fault in hardware is an erroneous state that is continuous and stable. Permanent faults in hardware are caused by the failure of the computing unit. We focus in this paper on the transient fault since, in most computing systems, the majority of errors are due to transient faults [9]. In the case of an energy-efficient system, reliability also means ensuring that the system will never be short of energy to ensure its treatment. Anticipation of possible cases of energy can, again, be implemented on the basis of the flexibility offered by the system at the level of the execution of the tasks.

In this work, we target the problem of real-time scheduling under reliability and energy constraints. Its about considering real-time tasks that have needs which are expressed on the one hand in terms of processing time and energy consumed by the processor and on the other hand in terms of the number of tolerated faults. A task configuration is energy overloaded, this means that the amount of energy consumed is greater than the amount of energy available. In addition, the amount of execution time requested is smaller than the available capacity, the system will therefore typically be able to meet all its deadlines or else catastrophic consequences will occur. A major question that needs to be answered is: how to schedule real-time tasks in case of energy where the system keeps reliable and able to tolerate up to $k$ faults.

To answer this question, a uniprocessor Earliest Deadline First (EDF) scheduler is first analyzed to derive an efficient and exact feasibility condition by considering energy management and fault-tolerance. Second, the proposed algorithm is designed to achieve energy autonomous utilization of the processor while meeting the task deadlines.

The rest of the paper is organized as follows. In the next section, we summarize the related work. In section 3 , we introduce the model and terminology. The fault tolerant speed schedule was then presented in section 4 . Section 5 presents the experimental results to demonstrate energy savings and Section 6 concludes the paper.

2. Related Work. Researchers in both academia and industry have resorted to various techniques to minimize energy consumption in computing systems. Among these, DVFS technique has risen as one of the best framework level methods for energy consumption. DVFS scheduling reduces the supply voltage and frequency when conceivable for preserving energy consumption. Subsequently, a great number of procedures considering the issue of limiting the energy consumption without jeopardizing the timing constraints on uniprocessor platforms are widely proposed in literature for different task models. Many of the previous work that studied the problem of energy efficient frameworks for real-time embedded systems employ the DVFS technique [4], [10], [11], [12], [13].

Yao et al. [10] developed a DVFS scheme for a set of aperiodic real-time tasks scheduled under EDF policy with a focus of minimizing dynamic power consumption for real-time systems. In [12], authors considered the temperate and leakage dependencies and proposed an efficient DVFS scheme to minimize the overall energy consumption while guaranteeing the timing constraints of a real-time system. Later in [13], we settle the hypothesis for enhancing energy saving in real-time systems, we proposed an energy-efficient scheduling algorithm for aperiodic tasksin real-time embedded systems. Specifically, we applied the DVFS technique to the concept of real-time process scheduling. Further, we proposed in [14] an energy guarantee scheduling and voltage/frequency selection algorithm targeting at real-time systems with energy harvesting capability. We show that our scheduler achieves capacity savings when compared to other schedulers.

On the other side, fault tolerance, and in general reliability, objectives are of paramount importance for embedded systems [15]: faults and failures can occur in real-time computing systems and can result in deadline violations and/or hardware errors. Since soft errors are more common in computing systems, most researches related to fault tolerance focus on soft errors. Such research efforts was done on scheduling techniques with the joint consideration of energy efficiency and fault tolerance. 
Zhu et al. [16] investigated the reliability problem of a real-time system as the probability to execute all tasks, in abscence or presence of faults. Following this, authors proposed a linear and an exponential model that can detect the effects of DVFS technique on the transient fault rate. They demonstrated that minimizing energy consumption through DVFS can reduce the system reliability. For this sake, they presented a recovery scheme to schedule a recovery for each scaled job to compensate the reliability loss caused by DVFS.

Melhem et al. [17] targeted the reliability problem for a set of periodic tasks scheduled under EDF on a monoprocessor with the restriction that there is at most one failure (i.e. $k=1$ ). Authors presented a checkpointing scheme that can reduce the fault-recovery overhead significantly at the cost of runtime overhead, this means by inserting checkpoints, which may potentially improve the system schedulability and leave more space for energy management. Zhang et al. [18] investigated the same problem but on fixed-priority real-time tasks. For this sake, authors proposed a DVFS scheme combined with checkpointing that is able to tolerate faults for a set of periodic tasks to minimize energy consumption.

More recently, Zhao et al. [19] proposed the Generalized Shared Recovery (GSHR) technique to reserve computing resources, which can be used by other tasks to enhance the energy efficiency. Later, this work was extended to be applied to a real-time periodic task model [15]. The proposed algorithms aim to determine the processor scaling factor and the reserved resources for every task to enhance the minimization of energy while still guaranteeing the reliability requirement at the task-level. The advantage of the GSHR scheduler comes from the fact that the reliability of the system can be increased when aplying the DVFS technique.

Recently, Han et al. developed effective scheduling algorithms that can save energy when considering that the proposed real-time system can tolerate up to $\mathrm{k}$ failures when scheduling a set of aperiodic tasks on a single processor under the EDF policy [20]. For this sake, authors proposed three algorithms: The first two algorithms are based on the previous work performed in [10]. The third algorithm extends the first two by considering that the computing resources are no longer reserved and hence better energy saving performance can be achieved. The main drawback of this work is that the problem of improving the system reliability in presence of failures cannot be solved by a simple modification to the work done in [10].

3. Model and Terminology. We consider the system model and their corresponding notations. Then, we present the problem formulation.

3.1. Task Model. We consider a set of $n$ independent aperiodic real-time jobs $\mathcal{J}=\left\{J_{1}, J_{2}, \cdots, J_{n}\right\}$, where $J_{i}$ denotes the $i^{\text {th }}$ job in $\mathcal{J}$ and is characterized by a three tuple $\left(a_{i}, c_{i}, d_{i}\right)$. The definition of these parameters are as follows:

- $a_{i}$ is referred to the arrival time, this means that the time when job $J_{i}$ is ready for execution.

- $c_{i}$ stands for the worst case execution time (WCET) under the maximum available speed $S_{\max }$ of the processor.

- $d_{i}$ is considered as the absolute deadline of job $J_{i}$.

We denote the laxity of the job $J_{i}$ by $d_{i}-\left(a_{i}-c_{i}\right)$. We consider that the job set $\mathcal{J}$ is said to be feasible in the real-time manner and under fault-free scenario. In other words, there exists a feasible schedule for $\mathcal{J}$ in abscence of energy considerations, where all deadlines in are respected.

3.2. Power and Energy Model. We assume the speed / frequency of the processor is equipped with a DVFS-enabled with $N$ discrete frequencies $f$ ranging from $f_{\min }=f_{1} \leq f_{2} \leq \cdots \leq f_{N}=f_{\max }$. We consider the notation processor speed $S_{N}$, or slowdown factor, as the ratio of the computed speed to the maximum processor speed, this means that $S_{N}=f_{N} / f_{\max }$ [13]. The CPU speed can be changed continuously in $\left[S_{\min }, S_{\max }\right]$. Consequently, when a job $J_{i}$ is executed under speed $S_{i}$, the worst case execution time of $J_{i}$ becomes equal to $c_{i} / S_{i}$.

For embedded systems, the processor and off-chip devices such as memory, I/O interfaces and underlying circuits mainly consume the major part of the energy [21]. In this paper, we distinguish between frequencydependent and frequency-independent power components. Specifically, we adopt the overall power consumption $(P)$ at a slowdown factor $S$ as follows:

$$
P=P_{\text {ind }}+P_{\text {dep }}=P_{\text {ind }}+C_{e f} S^{\alpha}
$$

Where $P_{\text {ind }}$ stands for the frequency-independent power that includes the constant leakage power and the power 
consumed by off-chip devices [20], which is independent of the system frequency and supply voltage. $C_{e f}$ is denoted as the effective switching capacitance. $\alpha$ is the dynamic power exponent, which is a constant usually larger than or equal to 2.

$P_{d e p}$ is considered to be the frequency-dependent active power, which includes not only the processor power, but also any power that depends on the processing speed $S$. Consequently, the energy consumption of a job $J_{i}$ that runs at the speed $S_{i}$, denoted as $E_{i}\left(S_{i}\right)$, can be expressed as:

$$
E_{i}\left(S_{i}\right)=\left(P_{i n d}+C_{e f} S_{i}^{\alpha}\right) \cdot \frac{c_{i}}{S_{i}}
$$

3.3. Energy Storage Model. Our system relies on an energy storage unit (battery or supercapacitor) with a nominal capacity, namely $C$, that corresponds to a maximum stored energy. The energy level of the battery must remain between two predefined boundaries, namely $C_{\min }$ and $C_{\max }$, where $C=C_{\max }-C_{\min }$. We consider that $C(t)$ stands for the energy level in the energy storage unit at time $t$. We state that the energy stored in the battery at any time is less than the storage capacity, that is

$$
C(t) \leq C \quad \forall t
$$

3.4. Fault Model. During the execution of an operation computing system, both permanent and transient faults may occur due to various reasons, like hardware defects or system errors. In this paper, we focus on transient faults since it has been shown to be dominant over permanent faults especially with scaled technology sizes [23].

We consider that the proposed system can afford a maximum of $k$ transient faults. The used system is usually able to detect faults when a job ends its execution. We assume that the energy and time overhead caused by fault detection, denoted as $E O_{i}$ and $T O_{i}$ respectively, are not negligible and are independent of the variations in the processor frequency.

Generally, there is not restriction on the occurrence of faults during the execution of jobs and multiple faults may occur when executing a single job [20]. The fault recovery scheme in this paper is based on re-executing the affected job. Consequently, $R_{i}$ stands for the maximum recovery overhead for executing a job $J_{i}$ under the maximum speed $S_{\max }$, which is equal to $c_{i}$, or $R_{i}=c_{i}$. When a fault occurs during any job execution, say $J_{i}$ , a recovery job is released having the same deadline $d_{i}$, which is subject to preemption as well.

3.5. Terminology. We now give some definitions we will be useful throughout the rest of this paper.

Definition 3.1. A schedule $\Gamma$ for a job set $\mathcal{J}$ is said to be valid if the deadlines of all jobs of $\mathcal{J}$ are met in $\Gamma$, starting with a storage fully charged [13].

Definition 3.2. A system is said to be feasible if there exists at least one valid schedule $\Gamma$ for $\mathcal{J}$ with a given energy source. Otherwise, it is infeasible [13].

In this paper, we consider that the limiting factors are not only time but are either, both time and energy, only energy or only time. We focus here on feasible systems only.

Formally, we introduce a novel terminology that is peculiar to energy constrained real-time computing systems.

Definition 3.3. A schedule $\Gamma$ for a job set $\mathcal{J}$ is said to be time-valid if the deadlines of all jobs of $\mathcal{J}$ are met in $\Gamma$, considering that $\forall 1 \leq i \leq n, E_{i}\left(S_{i}\right)=0$ [13].

DEFInItion 3.4. A system is said to be time-feasible if there exists at least one time-valid schedule $\Gamma$ for $\mathcal{J}$. Otherwise, it is infeasible [13].

Definition 3.5. A schedule $\Gamma$ for a job set $\mathcal{J}$ is said to be energy-valid if the deadlines of all jobs of $\mathcal{J}$ are met in $\Gamma$, considering that $\forall 1 \leq i \leq n, c_{i}=0$ [13].

Definition 3.6. A system is said to be energy-feasible if there exists at least one time-valid schedule $\Gamma$ for $\mathcal{J}$. Otherwise, it is infeasible [13].

3.6. Problem Formulation. We formulate the problem in this paper as follows: Given a set of real-time jobs $\mathcal{J}$ of $n$ independent aperiodic jobs $\mathcal{J}=\left\{J_{1}, J_{2}, \cdots, J_{n}\right\}$ having the following parameters: a release time, a worst-case execution time (WCET) and a deadline, that are executed on a DVS-enabled processor. Is it possible to minimize the overall energy consumption for all jobs in $\mathcal{J}$ along with the potential recovery operations without deadline violations under any fault scenario with at most $k$ 
transient faults? In our work, we use the ES-DVFS scheduling policy [13], which was proved to be optimal in minimizing the total energy consumption for uniprocessor systems under conventional or non-fault-tolerant analysis techniques. To answer this question, we have to find the CPU speed decisions (including the recoveries) in order to minimize the overall energy consumption within predefined timing constraints when no more than $k$ faults occur.

We consider that the processor is equipped with a set of discrete speed values for the whole time interval where $\mathcal{J}$ is executed as a speed schedule.

\section{Fault Tolerant Speed Schedule.}

4.1. Overview of the Scheduling Scheme. We develop an approach of a fault-tolerant DVFS scheduling for dynamic-priority real-time job set on uniprocessor systems to enhance energy savings while still guaranteeing the timing constraints. The proposed algorithm is based on the Energy Saving - Dynamic Voltage and Frequency Scaling (ES-DVFS) algorithm that we previously proposed in [13].

DEFINITION 4.1. A job set $\mathcal{J}$ is said to be $k$-fault tolerant if all jobs and potential recovery operations can be completed before their corresponding deadlines under any fault scenario with at most $k$ transient faults.

To better understand our approach and before proceeding, we first state some basic definitions and then briefly reiterate the general concept of ES-DVFS.

Definition 4.2. Given a real-time job set $\mathcal{J}$ of $n$ independent aperiodic jobs such that $\mathcal{J}=\left\{J_{1}, J_{2}, \cdots, J_{n}\right\}$.

- $\mathcal{J}\left(t_{s}, t_{f}\right)$ stands for the set of jobs contained in the time interval $\phi=\left[t_{s}, t_{f}\right]$, i.e jobs that are ready to be processed at time $t_{s}$ and with deadlines not more than $t_{f} . \mathcal{J}(\phi)=\left\{J_{i} \mid t_{s} \leq a_{i}<d_{i} \leq t_{f}\right\}$.

- $W(\phi)$ denotes the total amount of workload of jobs in $\mathcal{J}(\phi)$ in the time interval $\left[t_{s}, t_{f}\right]$, that means that the total worst case execution time of jobs that are completely contained in the time interval,

$$
W(\phi)=\sum_{J_{i} \in \mathcal{J}(\phi)} c_{i}
$$

- The processor load $h(\phi)$ over an interval $\phi=\left[t_{s}, t_{f}\right]$ is defined as

$$
h(\phi)=\frac{W(\phi)}{t_{f}-t_{s}}
$$

- The intensity of jobs in the time interval $\phi=\left[t_{s}, t_{f}\right]$, denoted as $I(\phi)$, is defined as

$$
I(\phi)=\max _{J_{j} \in \mathcal{J}(\phi)}\left(\frac{\sum_{d_{i} \leq d_{j}} c_{i}}{d_{j}-\left(t_{f}-t_{s}\right)}\right)
$$

- We consider that the fault-related overhead of a time interval $\phi=\left[t_{s}, t_{f}\right]$, denoted as $W_{k}(\phi)$ is

$$
W_{k}(\phi)=W_{r}(\phi)+W_{T O}(\phi)
$$

Where $W_{r}(\phi)$ stands for the worst-case reserved workload to be used in case of recovery, i.e. $W_{r}(\phi)=$ $k \times\left(R_{l}+T O_{l}\right)$ and $l$ represents the index of the job with the longest recovery time in $\mathcal{J}(\phi) . J_{l}=$ $\left\{J_{i} \mid \max \left(R_{i}+T O_{i}\right), J_{i} \epsilon \mathcal{J}(t \phi)\right\}$ and $W_{T O}(\phi)$ denotes the overhead imposed by fault detection from regular jobs, i.e.

$$
W_{T O}(\phi)=\sum_{J_{i} \in \mathcal{J}(\phi)} T O_{i}
$$

Further, $W_{k}(\phi) \geq W_{k-1}(\phi)$ for $k \geq 1$, since all the jobs' recovery have strictly positive execution times. For this sake, we restrict our analysis to the $k$-fault tolerance in such a way that there exists exactly $k$ faults when we investigate the worst-case reserved recovery of failures with at most $k$ faults. 
- The energy demand of a job set $\mathcal{J}$ on the time interval $\phi=\left[t_{s}, t_{f}\right]$ is

$$
g(\phi)=\sum_{t_{s} \leq r_{k}, d_{k} \leq t_{f}} E_{k}\left(S_{k}\right)
$$

Given a real-time job set $\mathcal{J}$, ES-DVFS was provably optimal in minimizing energy consumption in on-line energy-constrained setting by providing sound dynamic speed reduction mechanisms [13]. ES-DVFS produces an energy efficient technique by scaling down the processor frequency iwhile still guaranteeing the timing constraints. Using this framework, the slowdown factor of the ready jobs to be executed is not fixed in the used interval as the previous work in [10] but is dynamically adjusted on the fly. ES-DVFS is employed as follows:

- Step 1: Identify an interval $\phi=\left[t_{s}, t_{f}\right]$, add the ready jobs to the job queue $\mathcal{Q}$ and select the job $J_{i}$ with the highest priority.

- Step 2: Calculate the effective processor load $h(\phi)$ and intensity $I(\phi)$ using equations 4.2 and 4.3 respectively.

- Step 3: Set the speed $S_{i}$ of job $J_{i}$ to the maximum between $h(\phi)$ and $I(\phi)$.

- Step 4: In case of preemption, update $S_{i}$.

- Step 5: Remove job $J_{i}$ from the queue $\mathcal{Q}$.

- Step 6: Repeat step (1) - (6) until $\mathcal{Q}$ is empty.

Moreover, we proved that ES-DVFS provides an optimal speed schedule for a given job set $\mathcal{J}$.

Lemma 4.3. [13] An optimal speed schedule for a job set $\mathcal{J}$ is defined on a set of time intervals $\phi=\left[t_{s}, t_{f}\right]$ in which the processor maintains a constant speed $S_{i}=\max (I(\phi), h(\phi))$ where $h(\phi)$ and $I(\phi)$ are respectively the workload and intensity of jobs in $\phi=\left[t_{s}, t_{f}\right]$ and each of these intervals $\left[t_{s}, t_{f}\right]$ must start at $t_{s}$ and with deadlines at or earlier than $t_{f}$.

4.2. Concepts for the EMES-DVFS Model. ES-DVFS is proved to be optimal in case of fault-free conditions. Hence, To make the above ES-DVFS fault-tolerant, we adopt an approach, named MES-DVFS, that takes the fault recovery into consideration when calculating the effective processor load and intensity in any interval $\phi=\left[t_{s}, t_{f}\right]$, i.e. to replace $h(\phi)$ and $I(\phi)$ with $h_{m}(\phi)$ and $I_{m}(\phi)$ respectively, such that

$$
h_{m}(\phi)=\frac{\sum_{J_{i} \in \mathcal{J}(\phi)} c_{i}+k \times R_{l}}{d_{\max }-W_{T O}(\phi)-k \times T O_{l}}
$$

Where $d_{\max }$ is the longest deadline in $\mathcal{J}(\phi), l$ stands for the index of the job with the maximum recovery in $\mathcal{J}(t \phi)$ and $W_{T O}(\phi)$ stands for the overall fault-detection overheads for regular jobs as stated in Definition 4.2.

In addition, the intensity of the jobs in $\mathcal{J}(\phi)$ at current time $t$ is

$$
I_{m}(t)=\max _{J_{j} \in \mathcal{J}(\phi)}\left(\frac{\sum_{d_{i} \leq d_{j}} c_{i}+k \times R_{l}}{\left.d_{j}-t-W_{T O}(\phi)-k \times T O_{l}\right)}\right)
$$

Aydin, in [25], showed that the feasibility condition of scheduling a job set $\mathcal{J}$ by using EDF scheduler on a monoprocessor that can tolerate up to $k$ transient faults can be summarized as

THEOREM 4.4. [25] Given a real-time job set $\mathcal{J}$ that can tolerate a maximum of $k$ faults and with maximum processor speed $\left(S_{\max }=1\right)$, if for each interval $\left[t_{s}, t_{f}\right]$, we have

$$
\frac{\sum_{J_{i} \in \mathcal{J}\left(t_{s}, t_{f}\right)} c_{i}+W_{f t}\left(t_{s}, t_{f}\right)}{t_{f}-t_{s}} \leq 1
$$

When a fault is detected, and for the sake of enhancing the total savings for both the original jobs and their recovery copies, MES-DVFS runs the copy of the recovered job using a scaled processor speed $\left(S_{i} \leq S_{\max }\right)$. 
However, this may not be energy efficient since, in practice, the fault rate is usually very low.

An extended approach for MES-DVFS (we call it EMES-DVFS), is to execute the recovery copies under the maximum possible processor speed, usually at $S_{\max }$.

Hence, the intensity calculation of the jobs in $\mathcal{J}(\phi)$ can be modified correspondingly, as equation 4.10

$$
I_{e}(t)=\max _{J_{j} \in \mathcal{J}(\phi)}\left(\frac{\sum_{d_{i} \leq d_{j}} c_{i}}{d_{j}-t-W_{k}(\phi)}\right)
$$

Further, the effective processor load of the jobs in $\mathcal{J}(\phi)$ can also be modified correspondingly, as equation 4.11

$$
h_{e}(\phi)=\frac{\sum_{J_{i} \epsilon \mathcal{J}(\phi)} c_{i}}{d_{\max }-W_{k}(\phi)}
$$

4.3. Description of the EMES-DVFS Scheduler. In what follows, we consider a given set of $n$ jobs $\mathcal{J}=\left\{J_{1}, J_{2}, \cdots, J_{n}\right\}$ that can tolerate up to $k$ faults. Let $\mathcal{Q}(\phi)$ be the list of uncompleted jobs ready for execution at in the time interval $\phi=\left[t_{s}, t_{f}\right]$. We can formulate our EMLPEDF algorithm to obey the following rules:

Rule 1: The EDF priority order is used to select the future running jobs in $\mathcal{Q}(\phi)$.

Rule 2: The processor is imperatively idle in $\left[t_{s}, t_{s}+1\right)$ if $\mathcal{Q}(\phi)$ is empty.

Rule 3: The processor is imperatively busy in $\left[t_{s}, t_{s}+1\right)$ if $\mathcal{Q}(\phi)$ is not empty and $0<C\left(t_{s}\right) \leq C$. Hence, the following steps must be performed:

1. Select the job, say $J_{i}$ with the highest priority.

2. Calculate the effective processor load $h_{e}(\phi)$ and intensity $I_{e}(\phi)$ using equations 4.11 and 4.10 respectively.

3. Set the speed $S_{e i}$ of job $J_{i}$ to the maximum between $h_{e}(\phi)$ and $I_{e}(\phi)$.

Rule 4: If $S_{e i}<S_{\text {min }}$, then $S_{e i}=S_{\text {min }} \forall J_{i} \epsilon \mathcal{J}(\phi)$.

Rule 5: If job, say $J_{j}$ is released with $d_{j}<d_{i}$, then update $S_{e i}$ by Rule 3.

Rule 6: If job, say $J_{k}$ is released with $d_{k}>d_{i}$, then complete the execution of $J_{i}$.

Rule 7: If job, say $J_{k}$ is released with $d_{k}>d_{i}$, and $c_{k}>d_{k}-d_{i}$ then update $S_{e i}$ by Rule 3.

Rule 8: Calculate the energy consumption $E_{i}\left(S_{e i}\right)$ according to eq. (3.2).

Rule 9: Calculate the remaining energy in the battery at the end of the execution.

Rule 10: Remove job $J_{i}$ from the queue $\mathcal{Q}(\phi)$.

Rule 11: Repeat step (1) - (8) until the queue $\mathcal{Q}$ is empty.

4.4. Feasibility Analysis. When the job set $\mathcal{J}$ is feasible, it is not difficult to verify that $S_{e}(\phi) \leq$ $S_{m}(\phi)$ for a given interval $\phi=\left[t_{s}, t_{f}\right]$ since $\sum_{J_{i} \in \mathcal{J}(\phi)} c_{i}+W_{k}(\phi) \leq t_{f}-t_{s}$, where $S_{m}(\phi)$ and $S_{e}(\phi)$ are equal to $\max \left(I_{m}(\phi), h_{m}(\phi)\right)$ and $\max \left(I_{e}(\phi), h_{e}(\phi)\right)$ respectively.

More importantly, since EMES-DVFS can successfully schedule a given job set $\mathcal{J}$, then we can guarantee the feasibility of the resulted schedule. This is stated in the theorem below.

THEOREM 4.5. EMES-DVFS can guarantee that the deadlines of all jobs in $\mathcal{J}$ can be met as long as the following two constraints are satisfied: (1) no more than $k$ faults occur; (2) $\forall i \epsilon[1, n]$, where $n$ is the number of jobs in the job set $\mathcal{J}$, we have $S_{e i} \leq 1$ and $C(t)>0 \forall t$.

Proof. In EMES-DVFS, for a given time interval $\phi=\left[t_{s}, t_{f}\right]$, we can only execute jobs and their recovery copies included in the interval. For any job with higher priority, say $J_{h}$, and ready at time $t$, with possible execution overlapping with $\phi$, the EMES-DVFS scheduler must preempt the running job and begin executing $J_{h}$ and hence we will have a new slowdown factor that forces $J_{h}$ to finish within the interval $\phi$. Similarly, for 
any job with lower priority (e.g. $J_{l}$ ) with an execution time that can possibly overlap with $\phi$, the EMES-DVFS scheduler will update the processor speed and continue execution. $J_{l}$ is excluded from execution in the time interval $\phi$ and will be postponed to the next interval. Hence, scheduling decisions are only applied online when any of the following events occur: 1) Event 1: a new aperiodic job arrives and is added to the job queue. 2) Event 2: the current job is completely executed.

Hence, for proving this theorem, it is sufficient to prove that when the processor speed is set to $S_{e i}$, then we can guarantee that all jobs in $\phi$ are scheduled in the worst case scenario (i.e. against $k$ faults), as long as $S_{e i} \leq 1$ and the energy reservoir is not fully depleted $(C(\phi)>0)$. We prove this by contradiction. Consider that $J_{b}=\left(r_{b}, c_{b}, d_{b}\right) \in \mathcal{J}(\phi)$ misses its deadline when the processor speed is set to $S_{e i}$. Here, we have 2 cases:

Case 1: $J_{b}$ misses its deadline because of time starvation. Then we must be able to find a time $t \leq r_{b}$, such that for interval $\phi^{\prime}=\left[t, d_{b}\right]$, we have $\frac{W\left(\phi^{\prime}\right)}{S_{e i}}+W_{k}\left(\phi^{\prime}\right) \geq d_{b}-t$. Here we have 2 cases:

Case 1a: $J_{b}$ is not contained in the time interval $\phi$ (i.e. $d_{b} \geq t_{f}$ ). In this case, event 2 occurs, which means that the current running job, say $J_{i}$, will complete its execution without being preempted and job $J_{b}$ will be executed in the next time interval. This contradicts that $J_{b}$ misses its deadline.

Case 1b: $J_{b}$ is contained in the time interval $\phi$ (i.e. $d_{b} \leq t_{f}$ ). In this case, event 1 occurs, which means that the EMES-DVFS scheduler updates the processor speed schedule for all the ready jobs including the newly arrived one and consequently $S_{e i}$ is updated to another slowdown value $S_{e i}^{\prime}$ such that $S_{e i}^{\prime} \geq S_{e i}$ and $\phi^{\prime} \subseteq \phi$. Here we have 2 cases:

Case 1b1: Effective processor load $h_{e}\left(\phi^{\prime}\right)$ is greater than the intensity $I_{e}\left(\phi^{\prime}\right)$. This means that $S_{e i}^{\prime}\left(\phi^{\prime}\right)=$ $h_{e}\left(\phi^{\prime}\right)$. But, $h_{e}\left(\phi^{\prime}\right)$ is equal to $\frac{W\left(\phi^{\prime}\right)}{d_{\max }-W_{k}\left(\phi^{\prime}\right)}$ which is greater than or equal to $S_{e i}$. Since $S_{e i}^{\prime}\left(\phi^{\prime}\right) \geq S_{e i}$, then we have $\frac{W\left(\phi^{\prime}\right)}{S_{e i}^{\prime}} \leq \frac{W\left(\phi^{\prime}\right)}{S_{e i}}$. Take Eq. 4.11 into the right-hand side of the above inequality and add $W_{k}\left(\phi^{\prime}\right)$ to both sides. We get $\frac{W\left(\phi^{\prime}\right)}{S_{e i}^{\prime}}+W_{k}\left(\phi^{\prime}\right) \leq d_{b}-t \leq d_{\max }-t$. This violates the assumption that $J_{b}$ misses its deadline.

Case 1b2: Effective processor load $h_{e}\left(\phi^{\prime}\right)$ is smaller than the intensity $I_{e}\left(\phi^{\prime}\right)$. This means that $S_{e i}^{\prime}\left(\phi^{\prime}\right)=$ $I_{e}\left(\phi^{\prime}\right)$. But, $I_{e}\left(\phi^{\prime}\right)$ is equal to $\max _{J_{j} \in \mathcal{J}\left(\phi^{\prime}\right)}\left(\frac{\sum_{d_{i} \leq d_{j}} c_{i}}{d_{j}-t-W_{k}\left(\phi^{\prime}\right)}\right)$ which is greater than or equal to $S_{e i}$. Since $S_{e i}^{\prime}\left(\phi^{\prime}\right) \geq S_{e i}$, then we have $\frac{\sum_{d_{i} \leq d_{j}} c_{i}}{S_{e_{i}}^{\prime}} \leq \frac{\sum_{d_{i} \leq d_{j}} c_{i}}{S_{e_{i}}}$. Take Eq. 4.10 into the right-hand side of the above inequality and add $W_{k}\left(\phi^{\prime}\right)$ to both sides. We have $\frac{W\left(\phi^{\prime}\right)}{S_{e_{i}}^{\prime}}+W_{k}\left(\phi^{\prime}\right) \leq d_{b}-t$. This violates the assumption that $J_{b}$ misses its deadline.

Case 2: $J_{b}$ misses its deadline because of energy starvation. This means that $d_{b}$ is missed with energy demand $g\left(d_{b}\right)=0$. Then, we must find a time $t_{0} \leq d_{b}$ where a job with deadline after $d_{b}$ is released and no other job is ready just before $t_{0}$ and the battery is fully replenished i.e. $C\left(t_{0}\right)=C$. The processor is busy at least in the time interval $\phi^{\prime}=\left[t_{0}, d_{b}\right]$. Here we also have 2 cases:

Case 2a: No job with deadline greater than $d_{b}$ executes within the time interval $\phi^{\prime}$. This means that all the jobs that execute within $\phi^{\prime}$ have release time greater than or equal to $t_{0}$ and a deadline no more than $d_{b}$. The amount of energy needed to fully execute these tasks is $g\left(\phi^{\prime}\right)$. But since the processor is always busy in the time interval $\phi^{\prime}$, then jobs are executed with the minimum possible speed. Further, the energy reservoir is fully charged at $t_{0}$. Consequently, $g\left(\phi^{\prime}\right)<C\left(t_{0}\right)<C$. We conclude that all ready jobs within $\phi^{\prime}$ can be fully executed with no energy starvation, which contradicts the deadline violation at $d_{b}$ with $C\left(d_{b}\right)=0$.

Case 2b: At least one job, say $J_{m}$ is released within time interval $\phi^{\prime}$ and with with $r_{m}>r_{b}$. Here we have 2 cases:

Case 2b1: $J_{m}$ is released with $d_{m}<d_{b}$, therefore we have to update $S_{e i}$ by Rule 3 . Let $t_{2}$ be the latest time where $J_{m}$ is executed. As $d_{m}$ is lower than $d_{b}$ and jobs are executed according to preemptive EDF, we have $r_{m} \geq r_{b}$ and $J_{b}$ is preempted by the higher priority job $J_{m}$. Thus, the processsor speed must be updated, otherwise $d_{m}$ will be violated. Since the processor is busy all the times in $\left[t_{2}, d_{b}\right]$ and the job set $\mathcal{J}$ is timefeasible, then $S_{e m}$ will be the minimum speed for the execution of $J_{m}$ and consequently $g\left(t_{2}, d_{b}\right)<C\left(d_{b}\right)$. Hence, the amount of energy that $J_{m}$ require is at most $g\left(t_{2}, d_{b}\right)$. That contradicts deadline violation and 
$C\left(d_{b}\right)=0$.

Case 2b2: $J_{m}$ is released with $d_{m}>d_{b}$. We consider two cases: (i) $c_{m}<d_{k}-d_{b}$, hence $J_{b}$ will complete its execution (Rule 6) and the proof is therefore similar to case $2 a$. (ii) $c_{m}>d_{k}-d_{b}$, hence $S_{e b}$ must be updated (Rule 7) and the proof is therefore similar to case $2 b 1$.

Since all jobs in $\mathcal{J}$ are successfully executed within time intervals in EMES-DVFS and all running jobs within the corresponding time intervals are still schedulable when applying their corresponding speed, we prove the theorem.

口

We state the optimality of EMES-DVFS by proving that a job set $\mathcal{J}$ is feasible in a $k$-fault-tolerant if and only if all the jobs in $\mathcal{J}$ are executed without violating time and energy constraints. This violation is due to one of the two following reasons: either job, say $J_{i}$ lacks time (Lemma 4.6) or job $J_{i}$ lacks energy (Lemma 4.7 ) to complete its execution before or at deadline $d_{i}$. The time starvation occurs when deadline $d_{i}$ is missed with the energy reservoir not exhausted at $d_{i}$. On the other side, the energy starvation case is when the energy reservoir is fully depleted at $d_{i}$ and $J_{i}$ is not completed.

Further, the feasibility of the EMES-DVFS scheduler is guaranteed, which is formulated in Lemma 4.6 and Lemma 4.7.

LEMMA 4.6. A real-time job set $\mathcal{J}$ can be time-feasible in a $k$-fault-tolerant manner by EMES-DVFS if and only if all the jobs in $\mathcal{J}$ can still respect their deadlines when they are executed according to the processor speeds determined by EMES-DVFS for every time interval $\left[t_{s}, t_{f}\right]$.

Proof. Only if part. Directly follows Theorem 4.5.

If part. Suppose the contrary. Let us consider $\mathcal{J}(\phi)$ as the set of jobs contained in the time interval $\phi=\left[t_{s}, t_{f}\right]$, this means jobs that are ready to be processed at or after time $t_{s}$ and with deadlines at or earlier than $t_{f}$. We denote a fault pattern $f=\left\{f_{1}, f_{2}, \cdots, f_{n}\right\}$, where $f_{i}$ refers to the number of faults affecting job $J_{i}$ and its recovery. Hence, we say that $f$ is a $k$-fault pattern if the total number of faults is exactly $k$. Formally $h_{e}(\phi) \leq 1$ for all intervals $\left[t_{s}, t_{f}\right]$. However, there is a $j$-fault pattern $j \leq k$ (say $f^{j}$ ) resulting in deadline miss(es). Let us assume that the first deadline violation occurs at $t=d_{i}$ and that $t_{0}$ is the latest time preceding $d_{i}$ such that either the processor is idle or a job (recovery) of deadline $>d_{i}$ is executing.

We note that the time $t_{0}$ is well-defined in a way that it corresponds to a job release time. In addition, the processor is continuously busy executing jobs (recovery) in the time interval $\phi^{0}=\left[t_{0}, d_{i}\right.$ ). Now, let us denote $f^{0} \subset f^{j}$ be the subset of faults affecting jobs in the time interval $\phi^{0}$. Note that the number of faults in $f^{0}$ is obviously smaller than $k$. Since EDF is a work-conserving scheduling algorithm, this means that the processor is never kept idle unless there are no ready jobs, the deadline violation at $d_{i}$ and the above definition of $t_{0}$ imply that the available processor time in the interval time interval $\phi^{0}$ was not sufficient to accommodate the increase in the processor demand even there is no energy starvation in the interval $\phi^{0}$ (the battery is not fully replenished at time $\left.d_{i}\right)$. Consequently, we obtain $\sum_{J_{i} \in \mathcal{J}\left(\phi^{0}\right)} c_{i}+j \times R_{l}>d_{\max }-W_{T O}\left(\phi^{0}\right)-j \times T O_{l}$, where $j$ is the number of faults in $\phi^{0}$ and $l$ stands for the index of the job with the longest recovery time in $\mathcal{J}\left(\phi^{0}\right)$. But since $\sum_{J_{i} \in \mathcal{J}(\phi)} c_{i}+W_{k}(\phi) \leq d_{i}-t_{0}\left(h_{e}(\phi) \leq 1\right)$ and $W_{k}(\phi)>W_{k}\left(\phi^{0}\right)$ and $\sum_{J_{i} \in \mathcal{J}(\phi)} c_{i}>\sum_{J_{i} \in \mathcal{J}\left(\phi^{0}\right)} c_{i}$, we get $\sum_{J_{i} \in \mathcal{J}\left(\phi^{0}\right)} c_{i}+W_{k}\left(\phi^{0}\right) \leq d_{i}-t_{0}$ contradicting our assumption that a deadline violation occurs at $d_{i}$. $\square$

LEMma 4.7. A real-time job set $\mathcal{J}$ can be energy-feasible in a $k$-fault-tolerant manner by EMES-DVFS if and only if the deadlines of all the jobs in $\mathcal{J}$ can be met when they are executed based on the processor speeds determined by EMES-DVFS considering that for every time interval $\left[t_{s}, t_{f}\right], g\left(t_{s}, t_{f}\right)>0$.

Proof. Only if part. Directly follows Theorem 4.5.

If part. Suppose the contrary. Let us consider $\mathcal{J}(\phi)$ as the set of jobs contained in the time interval $\phi=\left[t_{s}, t_{f}\right]$. We also denote a fault pattern $f=\left\{f_{1}, f_{2}, \cdots, f_{n}\right\}$, where $f_{i}$ refers to the number of faults affecting job $J_{i}$ and its recovery. Hence, we say that $f$ is a $k$-fault pattern if the total number of faults is exactly $k$ and the energy in the reservoir is sufficient to execute all jobs in $\phi$. Formally $g(\phi)>0$ for all intervals $\left[t_{s}, t_{f}\right]$. However, there is a $j$-fault pattern $j \leq k$ (say $f^{j}$ ) resulting in deadline miss(es) due to energy starvation. Let us assume that the energy reservoir becomes empty at $t=d_{i}\left(C\left(d_{i}\right)=0\right)$ and that $t_{0}$ is the latest time preceding $d_{i}$ such that 
the processor is still executing a job (recovery) of deadline $<d_{i}$.

We note that the time $t_{0}$ is well-defined in a way that it corresponds to a job release time. In addition, the processor is continuously busy executing jobs (recovery) in the time interval $\phi^{0}=\left[t_{0}, d_{i}\right)$. Now, let us denote $f^{0} \subset f^{j}$ be the subset of faults affecting jobs in the time interval $\phi^{0}$. Note that the number of faults in $f^{0}$ is obviously smaller than $k$. Since EDF is a work-conserving scheduling algorithm, this means that the processor is never kept idle unless there are no ready jobs, the deadline violation at $d_{i}$ and the above definition of $t_{0}$ imply that the available energy in the reservoir in the time interval $\phi^{0}$ was not sufficient to accommodate the increase in the energy demand even there is no time starvation in the interval $\phi^{0}$, this means $\sum_{J_{i} \in \mathcal{J}\left(\phi^{0}\right)} c_{i}+j \times R_{l} \leq d_{\text {max }}-W_{T O}\left(\phi^{0}\right)-j \times T O_{l}$, where $j$ is the number of faults in $\phi^{0}$ and $l$ stands for the index of the job with the maximum recovery time in $\mathcal{J}\left(\phi^{0}\right)$. But since the job set $\mathcal{J}(\phi)$ is feasible, then $g(\phi)>0$. In addition, the energy demand in $\phi$ is greater than the energy demand in $\phi^{0}$, since the number of faults in $\phi(k)$ is more than that in $\phi^{0}(j)$, i.e. $g(\phi)>g\left(\phi^{0}\right)$. Hence, we get $g(\phi)>g\left(\phi^{0}\right)>0$ contradicting our assumption that a deadline violation occurs at $d_{i}$ because of energy starvation.

Now, we may draw Theorem 4.8, a major result of optimality for uniprocessor scheduling in a $k$-fault-tolerant manner by EMES-DVFS with time and energy constraints.

THEOREM 4.8. The EMES-DVFS scheduling algorithm is optimal for a $k$-fault-tolerant model.

Proof. According to Lemma 4.6, EMES-DVFS can schedule a given set of jobs $\mathcal{J}$ in a $k$-fault-tolerant manner, without violating timing constraints when the energy demand is lower than the maximum energy that is available in the reservoir. According to Lemma 4.7, EMES-DVFS can schedule a given set of jobs $\Gamma$ in a $k$-fault-tolerant manner, without violating energy constraints when the processor demand is cannot exceed the maximum available processor time that could be available in any given time interval. As a conclusion, if EMESDVFS can schedule a given set of jobs $\mathcal{J}$ for a given time or/and energy constraints without time starvation and energy starvation, are the only two reasons for deadline violations, then we conclude that EMES-DVFS is optimal. $\mathrm{c}$

5. Evaluation. In this section, we study the performance of four scheduling algorithms: EMES-DVFS, MES-DVFS, NPM and LPSSR proposed in [20]. NPM scheme executes jobs with maximum frequency and does not scale down the voltage/frequency.

We developed a discrete event-driven simulator in $\mathrm{C}$ that generates a job set $\mathcal{J}$ where the number of jobs varies from 10 to 50 . The simulation is repeated 100 times for the same number of jobs.

For the sake of clarity, we use NPM as a reference schedule that represents the schedule of given set of jobs $\mathcal{J}$ without incorporating DVFS. This means that all jobs or recoveries in $\mathcal{J}$ are executed under the maximum processor speed $S_{\max }$. We consider that all the plotted energy consumptions are normalized to NPM. However, to give LPSSR a fair chance, we consider the same parameters as used in [20]. Hence, we consider the following parameters: We assumed that $\alpha=2, C_{e f}=1, P_{\text {ind }}=0.05$, and $S_{\text {min }}$ is set to 0.25 .

The proposed algorithms are tested with randomly generated job sets as follows: the choice of the arrival time $a_{i}$ and the relative deadline of each job $J_{i}$ is assumed to be distributed uniformly in the time interval $[0 s, 100 s]$ and $[50 s, 100 s]$ respectively. Moreover, the worst case execution time $c_{i}$ is randomly generated such that $c_{i}<d_{i}$. The timing and energy overhead of detecting faults is considered as $10 \%$ of the WCET and its energy consumption respectively. As for the fault arrival rate, we consider 2 cases: safety-critical real-time system with range of $10^{-10}$ to $10^{-5}$ /hour or in harsh environment with a range between $10^{-2}$ and $10^{2}$ /hour.

All simulation results are computed on a discrete DVFS processor that operates on 8 frequency levels $\{1.00,0.86,0.76,0.67,0.57,0.47,0.38,0.28\}$ as in the PentiumM processor.

We report here two sets of experiments. The first set is designed to show the energy consumption of the 4 approaches by varying the number of jobs. In the second experiment set-up, we compare the energy consumption by varying the number of faults.

5.1. Experiment 1: Energy Consumption by Varying the Number of Faults. In this set of simulations, we evaluated the impact of the number of faults on energy savings. In this experiment, we took a fixed number of jobs equal to 15 and the number of tolerated faults varies between 1 and 10. Again, we repeat the experiment for 100 times with different generated test cases but with the same number of faults. We took then the average results, which are shown in figure 5.1. 


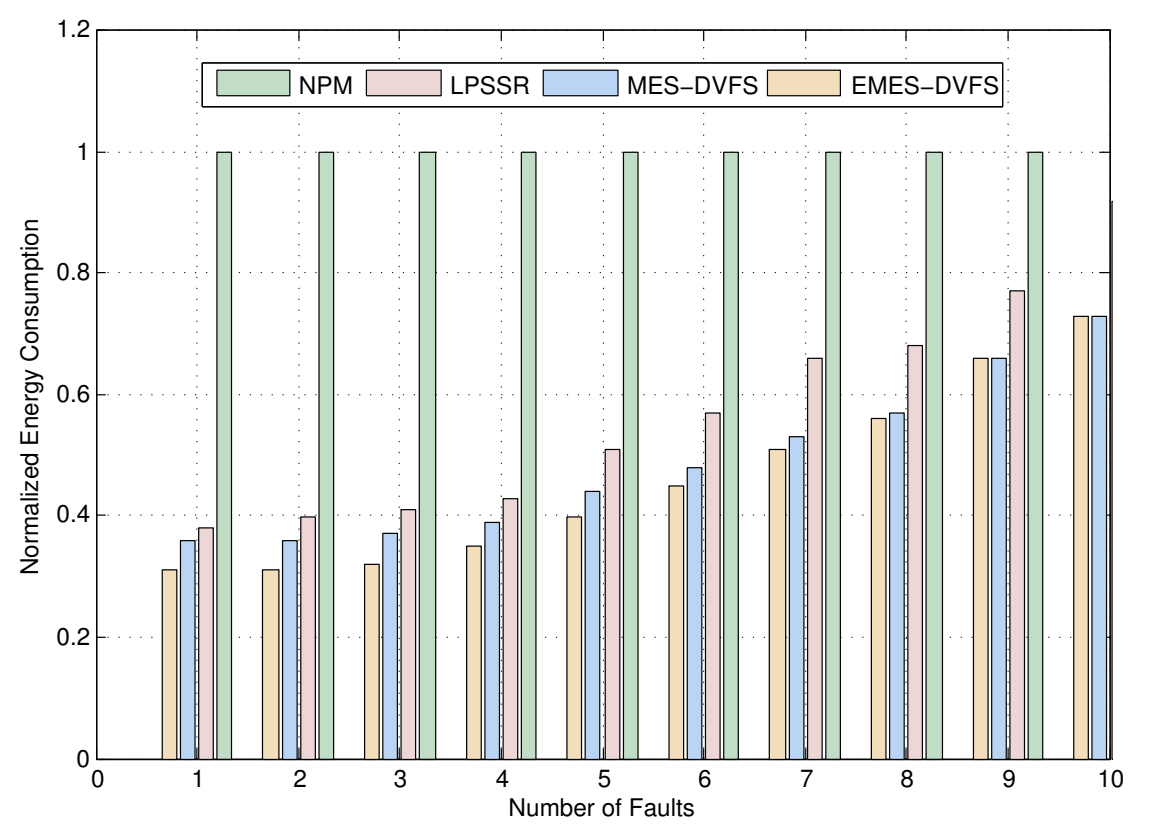

FIG. 5.1. Energy savings by varying the numbers of faults.

From the figure 5.1, we can find that EMES-DVFS and MES-DVFS can achieve energy savings compared to LPSSR and NPM. Clearly, we find that the energy consumptions by the four schedulers increase rapidly as the number of faults increases, since more expected energy may be consumed due to the increased number of recovery jobs being executed, which in turn limits the maximum amount of dynamic slack used. However, as the number of faults increases, the energy consumption in EMES-DVFS and EMES-DVFS grows but less dramatically.

As illustrated in figure 5.1, the EMES-DVFS and MES-DVFS approaches attain respectively around $19 \%$ and14\% more energy saving than LPSSR. The reason is that the optimal dynamic slack time to minimize the expected energy consumption is used to the maximum extent by employing speed assignment on the fly. On the contrary, LPSSR is significantly affected when we increase the number of system faults and the system consumes of about $22 \%$ additional energy when we increase the fault occurrences from 1 to 10 . On the other hand, EMES-DVFS could tolerate up to 5 times more faults with same energy as consumed by LPSSR.

As a conclusion, the advantage of our approaches (EMES-DVFS and MES-DVFS) over the other two (LPSSR and NPM) in terms of energy savings is evident in this experiment where EMES-DVFS and MESDVFS can still guarantee tolerance even under 10 faults and with more energy saving than LPSSR and the energy savings drops around 19\% under LPSSR when we compare it with EMES-DVFS.

5.2. Experiment 2: Energy Consumption by Varying $P_{\text {ind }}$. In this experiment, we study the impact of frequency-independent power $P_{i n d}$ on energy savings. $P_{i n d}$ varies between $[0,0.3]$ for each job and the number of jobs is fixed at 15. According to figure 5.2, the larger the $P_{\text {ind }}$, the higher the energy consumption. This is due to the fact that as the $P_{\text {ind }}$ increases, the contribution of frequency independent energy consumption becomes more dominant, the energy-efficient frequency increases and consequently DVFS has fewer opportunities to be applied. Even under this situation, EMES-DVFS still has the best performance in terms of energy consumption (EMES-DVFS attains approximately $18 \%$ more energy saving than LPSSR).

5.3. Experiment 3: Percentage of feasible Job Set. In this experiment, we take interest in the percentage of feasible job set that respect their deadlines with the four scheduling algorithms by varying the energy storage capacity. From this experiment, we can deduce two measures. The first one gives us an indication about the percentage of time during which all deadlines are still respected. The second one gives, for each 


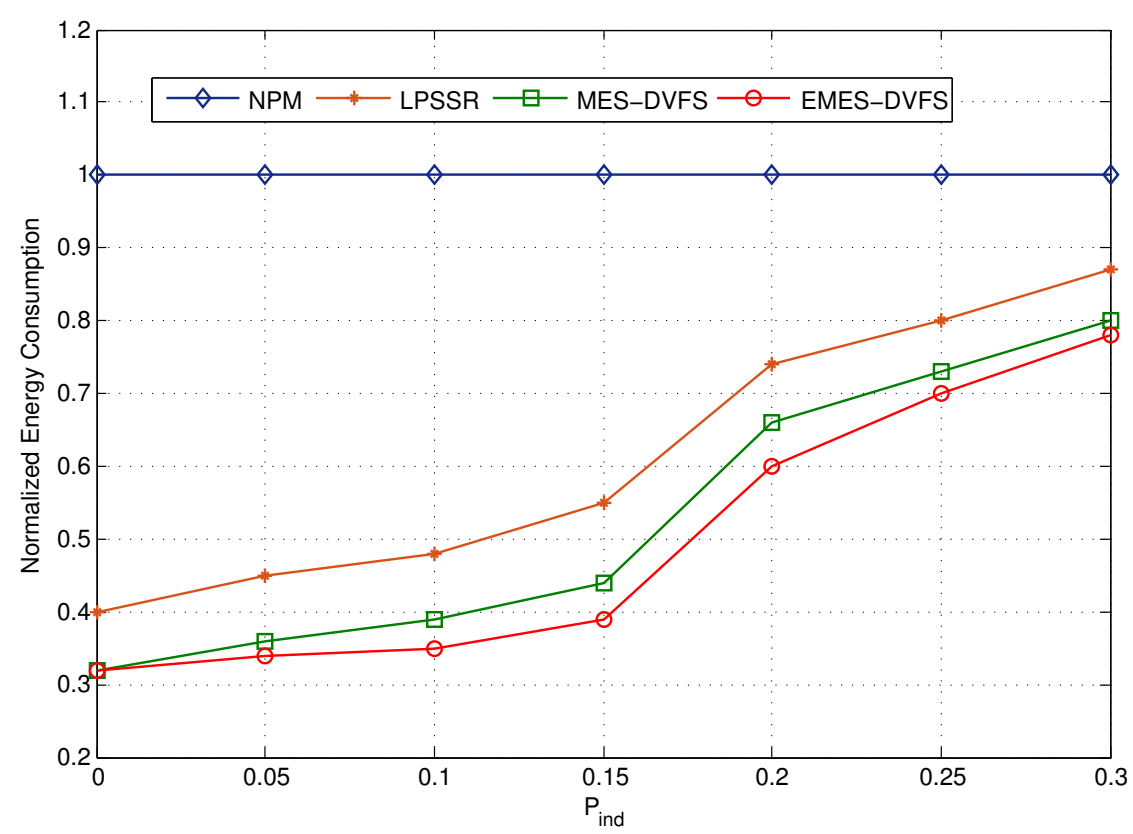

FIG. 5.2. Energy savings by varying $P_{\text {ind }}$.

approach and for a given processor load, the minimum size of the storage that ensures time and energy feasibility. We report here the results of two simulation studies where the processor load is set to 0.4 and 0.8 , respectively.

(a) Processor load $=0.4$

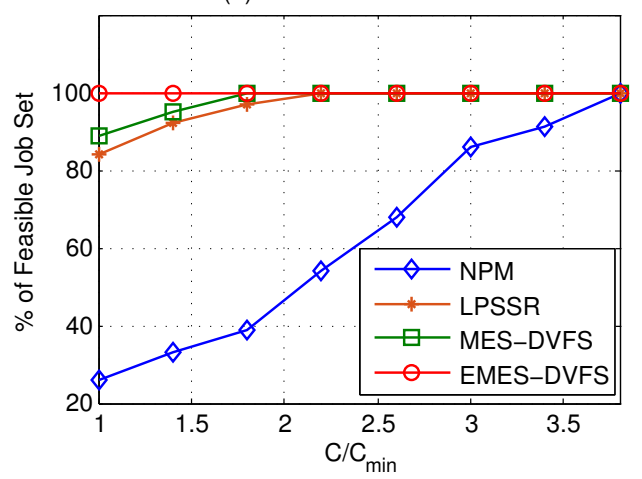

(b) Processor load $=0.8$

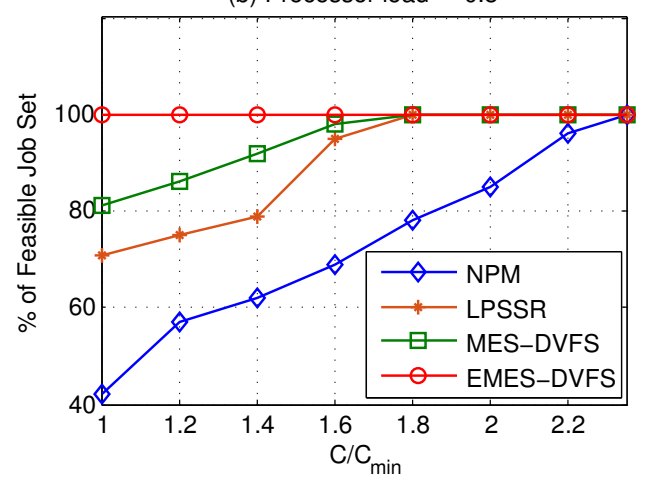

Fig. 5.3. Percentage of feasible job set. (a) Low processor load. (b) High processor load.

Figure 5.3 depicts the percentage of feasible job sets that meet their deadlines over the energy storage capacity $C$. For each job set, we compute the minimum storage capacity $C_{\min }$ which permits achieving time and energy feasibility under EMES-DVFS. We then begin to increase the energy storage capacity till the 4 approaches achieve neutral operation.

Under low processor load (figure 5.3a), it is observed that 100\% of job sets meet their deadlines under EMES-DVFS when the energy storage capacity is 4510 energy units, i.e. $C=C_{\min }=4510$ energy units. We start then to increase $C$ till it reaches 8118 where MES-DVFS becomes feasible. this means that means that EMES-DVFS can provide the same level of performance with a storage unit which is about 1.8 times less. The increase in the storage capacity will continue to increase till LPSSR and NPM becomes feasible where the energy storage unit must be respectively more than 2.2 and 3.8 times bigger with LPSSR and NPM to maintain zero 
deadline miss, compared with EMES-DVFS.

The results for high processor load (figure 5.3b) follow the same trend. Unlike the previous experiment, the relative performance gain of EMES-DVFS in terms of capacity savings is decreasing when the processor load is increasing. EMES-DVFS obtains respectively capacity savings of about $37 \%, 44 \%$ and $57 \%$ compared with MES-DVFS, LPSSR and NPM.

It is important here to note that the four approaches require exactly the same storage size when the processor load is equal to 1 since the processor is continuously busy and there is no chance to apply DVFS.

In summary, this experiment points out that the proposed EMES-DVFS approach is very effective in reducing deadline miss rate and storage size even under high processor load. And lower is the processor load rate, higher is the capacity saving and our approach will then outperform the others by a high amount of energy savings.

6. Conclusions. In this paper, we presented and evaluated a novel approach, which aims to minimize energy consumption when scheduling a set of real-time jobs that can tolerate up to $k$ transient faults while still respecting time and energy constraints. We explore the reserved slacks generated during run-time to the maximum extent in such a way that all the available slack time is used for energy reduction, which is carried out using dynamic voltage and frequency scaling (DVFS). Under this notion, we propose an algorithm that estimates an optimal speed reduction mechanism which maintains feasibility within predefined timing constraints when no more than $k$ faults occur.

Our scheduler dynamically adjusts the jobs' slowdown factors by utilizing run-time slacks which may be increased for recovery demands of the system. It differs from the existing approach where job frequencies assignments are predetermined, and hence it is more flexible and adaptive in minimizing energy consumption while still keeping the systems reliability at a desired level. In addition, we presented two feasibility tests for recovery schemes under variable processor speed which decouples the time and energy constraints. The experimental results demonstrate that the proposed algorithm can significantly improve the energy savings compared with the previous works.

For future work, we will explore the adaptation of the proposed approaches to fixed priority environments in real-time energy harvesting systems.

Acknowledgments. This work was fully supported by a research grant from the Lebanese University.

\section{REFERENCES}

[1] E. Bini And G. C. Buttazzo. Measuring the performance of schedulability tests. Real-Time Systems, 30(1-2):129-154, May 2005.

[2] D. Brooks, P. Bose, S. Schuster, H. Jacobson, P. Kudva, A. Buyuktosunoglu, J.-D. Wellman, V. Zyuban, M. Gupta, AND P. CoOK. Power-aware microarchitecture: Design and modeling challenges for next-generation microprocessors. Micro, IEEE, 20(6):26-44, Nov 2000.

[3] R. Gupta. Dynamic voltage scaling for system-wide energy minimization in real-time embedded systems. Proceedings of the International Symposium on Low Power Electronics and Design ISLPED '04, pages 78-81, Aug 2004.

[4] H. Aydin, R. Melhem, D. Mosse, and P. Mejia-Alvarez. Power-aware scheduling for periodic real-time tasks. IEeE Transactions on Computers, 53(5):584-600, 2004.

[5] H. Aydin, V. Devadas, And D. Zhu. System-level energy management for periodic real-time tasks. In Proc. of IEEE RealTime Systems Symposium (RTSS), pages 313322, Dec. 2006.

[6] V. Devadas and H. Aydin. On the interplay of dynamic voltage scaling and dynamic power management in real-time embedded applications. In Proc. ACM Conference on Embedded Systems Software (EMSOFT'08), 2008.

[7] D. Siewiorek And R. Swarz. Reliable Computer Systems: Design and Evaluation. Natick, MA: A. K. Peters, Ltd., 1998.

[8] Srinivasan J, Adve SV, Bose P, Rivers J, Hu CK. Ramp: A model for reliability aware microprocessor design. IBM Research Report, RC23048, 2003.

[9] Castillo X, McConnel SR, Siewiorek DP. Derivation and calibration of a transient error reliability model. IEEE Trans Comput 31:658671, 1982.

[10] F. Yao, A. Demers, And S. Shenker. A scheduling model for reduced cpu energy. In Foundations of Computer Science, Proceedings., 36th Annual Symposium on, pages 374-382, oct 1995.

[11] Y. Zhang, K. Chakrabarty, and V. Swaminathan. Energy-aware fault tolerance in fixed-priority real-time embedded systems. In Proceedings of the 2003 IEEE/ACM international conference on Computer-aided design, ICCAD'03, 2003.

[12] H. HuANG AND G. QuAn. Leakage aware energy minimization for real-time systems under the maximum temperature constraint. In Design, Automation Test in Europe Conference Exhibition (DATE), 2011, pages 1-6, March, 2011. 
[13] Hussein El Ghor, E. M. AgGoune. Energy efficient scheduler of aperiodic jobs for real-time embedded systems, International Journal of Automation and Computing, pages 1-11, 2016.

[14] Hussein EL GHOR, Maryline CHETTO. Energy Guarantee Scheme for Real-time Systems with Energy Harvesting Constraints. International Journal of Automation and Computing, to appear.

[15] Baoxian Zhao, Hakan Aydin and Dakai Zhu. Energy Management under General Task-Level Reliability ConSTRAINTS. IEEE 18th Real Time and Embedded Technology and Applications Symposium, 2012.

[16] Zhu D, Melhem R, Mosse D. The effects of energy management on reliability in real-time embedded systems. In: ICCAD '04, Proceedings of the 2004 IEEE/ACM International conference on Computer-aided design, IEEE Computer Society, Washington, DC, pp 35-40, 2004.

[17] R. Melhem, D. Mosse, and E. Elnozahy. The interplay of power management and fault Recovery in Real-time Systems, IEEE Transactions on Computers, 53(2):217-231, Feb 2004.

[18] Y. Zhang and K. Chakrabarty. A unified approach for fault tolerance and dynamic power management in fixed-priority real-time embedded systems. IEEE Transactions on Computer-Aided Design of Integrated Circuits and Systems, 25(1):111125 , jan. 2006.

[19] B. Zhaо, H. Aydin, And D. Zhu. Generalized reliability-oriented energy management for real-time embedded applications. In 48th ACM/EDAC/IEEE Design Automation Conference (DAC), pages 381-386, june 2011.

[20] Qiushi Han, Linwei Niu, Gang Quan, Shaolei Ren and Shangping Ren. Energy efficient fault-tolerant earliest deadline first scheduling for hard real-time systems. Real-Time Systems 50:592-619, 2014.

[21] T. D. Burd and R. W. Brodersen. Energy efficient cmos microprocessor design. In Proc. of The HICSS Conference, Jan. 1995.

[22] J. W. S. W. Liu. Real-time Systems, NJ, USA: Prentice Hall, 2000.

[23] P. Hazucha and C. Svensson. Impact of cmos technology scaling on the atmospheric neutron soft error rate. IEEE Trans. on Nuclear Science, 47(6) 2586-2594, 2000.

[24] Pradhan DK. Fault-tolerant computer system design. Prentice-Hall Inc, Upper Saddle River, 1996.

[25] Aydin H. Exact fault-sensitive feasibility analysis of real-time tasks. IEEE Trans Comput 56(10):1372-1386, 2007.

\section{Edited by: Dana Petcu}

Received: Sep 4, 2018

Accepted: Nov 3, 2018 


\title{
MODELLING AND SIMULATION OF GPU PROCESSING IN THE MERPSYS ENVIRONMENT
}

\author{
TOMASZ GAJGER ${ }^{\ddagger}$ AND PAWEE CZARNUL TH $^{\dagger}$
}

\begin{abstract}
In this work, we evaluate an analytical GPU performance model based on Little's law, that expresses the kernel execution time in terms of latency bound, throughput bound, and achieved occupancy. We then combine it with the results of several research papers, introduce equations for data transfer time estimation, and finally incorporate it into the MERPSYS framework, which is a general-purpose simulator for parallel and distributed systems. The resulting solution enables the user to express a CUDA application in a MERPSYS editor using an extended Java language and then conveniently evaluate its performance for various launch configurations using different hardware units. We also provide a systematic methodology for extracting kernel characteristics, that are used as input parameters of the model. The model was evaluated using kernels representing different traits and for a large variety of launch configurations. We found it to be very accurate for computation bound kernels and realistic workloads, whilst for memory throughput bound kernels and uncommon scenarios the results were still within acceptable limits. We have also proven its portability between two devices of the same hardware architecture but different processing power. Consequently, MERPSYS with the theoretical models embedded in it can be used for prediction of application performance on various GPUs.
\end{abstract}

Key words: Performance simulation, Simulation environment, GPGPU, CUDA

AMS subject classifications. 68M20, 65Y05, 68U20

1. Introduction. Graphics processing units (GPUs) are highly data-parallel devices that are nowadays ubiquitously used for a variety of applications by employing the GPGPU paradigm. GPGPU stands for generalpurpose computing on a GPU and refers to the GPU being used to execute tasks, which are not necessarily related to graphics - general purpose tasks, e.g. numerical algorithms, neural networks training, data analysis, and many more. In order to use what the GPU offers effectively, the programmer needs to write a dedicated application. Furthermore, the GPU differs greatly from the CPU both in terms of a hardware design and processing model. It would be of a great help to such a programmer to have a tool that allows creation of a theoretical model of an application and provides means to assess it in terms of computational performance, scalability and behaviour on different hardware units. This becomes even more important when the GPUs are used in highly parallel and distributed environments such as HPC clusters, grids or volunteer computing networks. What is more, according to the recent TOP500 [1] list of the fastest supercomputers, the accelerators (mostly NVIDIA GPUs) are an important component used in $22 \%$ of these systems.

Considering NVIDIA's dominance in this field, the size of the CUDA community and maturity of available software tools, we have decided to focus our efforts on the NVIDIA GPUs. Nevertheless, we have developed a generic solution for simulation of running applications on GPUs. Use of a simulator equipped with a proper theoretical model, such as MERPSYS described later, may be beneficial in many ways, one can use it to analyse the behaviour of an application in hardware setups which may be unavailable for testing purposes, such as before purchasing. It also allows to exceed limits imposed by the hardware, e.g. assess the relation between execution time and data size for very large data sets. Despite providing estimations for application execution time, a simulator may also compute the predicted energy consumption or failure chance. These values may be then used for multi-criteria optimisation [44], including energy efficiency and reliability. For a GPU, a simulator can allow easy assessment of application execution using various configurations such as grid configuration or application parameters. When searching for an appropriate theoretical model one should consider only these of acceptable (preferably as high as possible) accuracy, but this is not the only expected trait. Ease of use, the possibility to extend and customise the model are important as well and lastly, the closer the model resembles the actual processing that happens on the GPU, the better. The complexity of the GPU hardware and abundance of internal parallelism makes such a theoretical model harder to develop, but as we will further show in Section 4.1,

\footnotetext{
*tomasz.gajger@pg.edu.pl

${ }^{\dagger}$ pczarnul@eti.pg.edu.pl

$\ddagger$ Department of Computer Architecture, Faculty of Electronics, Telecommunications and Informatics, Gdansk University of Technology, Narutowicza 11/12, 80-233 Gdańsk, Poland
} 
this is still a manageable task. We have analysed existing solutions, based on which we propose a performance model for a GPU.

In this paper we contribute by incorporation of modelling parallel processing on a GPU into an existing simulator - MERPSYS [15], validate the model against real results for a parallel GPU-enabled application with various launch configurations on three different GPUs and emphasise benefits from using MERPSYS for simulation using various GPUs from its database. Details of our contribution are described in the context of introduced models in Section 3.3. The paper is organised as follows: Section 2 explains important aspects related to the GPU processing and briefly describes the MERPSYS framework, Section 3 lists related work and motivations for developing the modelling solution. In Section 4, we propose our own solution, explain it in detail, and show its implementation in Section 5. Section 6 describes our testbed, testing methodology, validation of the model, and presents the results. Finally, Section 7 summarises results and outlines the proposed direction of future research.

\section{Background.}

2.1. GPU architecture and programming model. GPU is a many-core device that exemplifies a SIMD (Single Instruction Multiple Data) architecture type per Flynn's taxonomy [32]. Although the GPU itself does not contain vector units but rather simple cores capable of executing one instruction on a single operand at a time (or two instructions if we consider FMAC operations), it acts in a very similar manner. All the threads comprising a single work group (called warp) in a single clock cycle execute the same instruction on different operands, that is why NVIDIA describes this architecture as SIMT [39] (Single Instruction Multiple Threads). This term is obviously a direct derivative of SIMD but expresses the internal architecture of the device more clearly - multiple threads executing an instruction instead of a single thread executing it on a wide register containing multiple operands. GPU is perfectly suited for handling tasks that are highly data parallel and compute intensive, yet simple in terms of the control logic.

A CUDA program is heterogeneous in its nature and consists of two parts: one that executes on the CPU and one that is offloaded to the GPU. Upon program launch the code is executed by the CPU until a kernel invocation is encountered. When it happens, two important parameters are specified: grid size and block size [37]. As the grid may be comprised of many threads, it is logical that only a limited number will be loaded to the Streaming Multiprocessors (SMs) at a given time, allocation is done at the block level and once a block finishes its execution a new one will be scheduled. This process continues until all of the blocks are processed [19].

Without going into deeper details, from our perspective the following facts are important:

1. SM may hold a limited number of blocks at a time;

2. blocks have their requirements for shared memory and registers which need to be allocated from the pool available on the SM;

3. threads are executed in groups of 32 , called warps;

4. there are constraints regarding maximum number of active warps as well as rules affecting warps execution;

5. SM can perform zero-overhead context switches between warps.

The above directly affect the capability of the SM to execute the instructions effectively. If the number of active warps is not large enough to cover the stalls (caused by memory accesses, conditional branches resulting in warp divergence, etc.) then performance will be suboptimal [45]. The process of calculating the number of threads that may be loaded to a single SM considering shared memory and register constraints is called occupancy calculation. Shortly speaking, if the occupancy is poor then likely the performance of the application will decrease. It should be noted, however, that increasing occupancy may not lead to any performance gains or may even result in a performance degradation [46] because of an increased contention for shared resources as was experimentally proven in [6]. It is also possible to achieve optimal performance with a very low occupancy if thread granularity (number of instructions executed per thread) is sufficient [50]. Given all these, it is clear that the effectiveness of warps execution depends on the characteristics of the specific kernel.

2.1.1. Compiled CUDA code. CUDA code can be compiled into two representations: PTX (Parallel Thread Execution) or SASS (Shader Assembly). PTX is a model of a virtual machine and a definition of ISA to be used with this machine, it exposes GPU to the higher layers as a data-parallel computing device. PTX 
code is an intermediate representation of a program and an abstraction over GPU architecture that is not tied to any hardware type, thus it does not directly resemble the instructions that are executed by the GPU. It is ultimately translated into a machine code (cubin object) either by nvcc in a separate compilation step or by leveraging just-in-time compilation by the CUDA runtime driver upon being scheduled for launch on a specific device [38]. This allows a high level of interoperability between varying GPU architectures. SASS is the machine code for GPU, generated for a specific architecture and maps directly to instructions being processed by the functional units. It can be either generated directly by nvcc during the compilation process or, as it was already mentioned, generated on-the-fly from PTX source code.

2.2. MERPSYS. Developed at the ETI faculty of Gdansk University of Technology, MERPSYS ${ }^{1}[13,15]$ is a simulation environment for distributed systems that allows its users to predict execution time, power usage and failure probability of their applications. The application is described in a form of Java code imbued with special functions implementing communication (MPI-like) and computation primitives. The system is modelled as a hierarchical structure of components representing computational devices and interconnects, each of them having its own characteristics defined. It consists of four software components: GUI, server, database and simulator. Block based representation [12] is employed as a theoretical concept behind the application model, the environment provides a set of extensions to the Java language that allow to define computation and communication blocks while preserving the original structure of the code. An application model can be expressed in Java with additional message passing communication primitives using properly parametrized (input data size, operation type, etc.) MERPSYS-specific constructs in place of actual computation or communication code.

A system model is created by selecting hardware components and placing them in the editor's workspace. It may consist of several nested levels, e.g., two machines connected using an InfiniBand network and inside each of them a GPU and a processor connected via a PCIe bus. The hardware model is a term used to describe the characteristics of a given hardware setup. A model has special functions associated with it that reflect, e.g.: power usage equation for an active or idle component, communication time for point-to-point, scatter or gather operations, etc. These functions are then used by the simulator to calculate the effective time taken by various operations. Hardware components have attributes that describe their characteristics. A database of hardware components is provided, that can be extended with new records. It should be further noted that the hardware model is sometimes referred to as a computational model. For each computational component a user defines an arbitrary number of labels that are used to tie application and hardware models together. They are used in the application model to mark parts of the code representing distinct processes or threads and allow the scheduler to map them to the hardware components. A simulation configuration screen also allows to define attributes that are passed to the application model, an example of such may be data size, block configuration of a GPU, etc. A request to launch a simulation is passed from GUI to the server which in turn directs it to one of the simulators connected to one of its simulation queues [13]. The simulation queue to be used may be specified as well.

MERPSYS was successfully used by its authors to model execution time, energy consumption and reliability of divide-and-conquer (DAC [18, 13]) and geometric single program multiple data (SPMD [13]) applications [16], K-means algorithm [17] and volunteer computing systems [14]. For a more detailed description providing better insight into the whole MERPSYS environment see [15].

\section{Related Work.}

3.1. GPU performance models. When considering performance models targeted specifically at GPUs, several approaches are to be distinguished, three of the most common are [30]: analytical methods, quantitative and compiler-based methods, statistical and machine learning methods. The first kind focuses on creation of a theoretical model for a kernel execution expressed as set of equations and feeding it with parameters obtained by direct analysis of the kernel's code and the underlying hardware. Amaris et al. have proposed an analytical model [2] based on BSP [49], that estimates kernel execution time using an equation combining global and shared memory access times, with computation time and available GPU hardware resources. Another model [23] focuses on highly parallel aspects of GPU processing, discerning ones originating from parallel execution of

\footnotetext{
${ }^{1}$ http://merpsys.eti.pg.gda.pl
} 
warps and memory accesses. It enumerates as many as 21 distinct parameters obtained via source code analysis, microbenchmarking, or defined by user. These are then used to estimate kernel's execution time.

The model proposed by Volkov [51] leverages Little's Law by defining the parallel process using three metrics: warp latency bound, warp throughput bound and occupancy. Execution of a kernel is modelled at a warp level in the scope of a single SM with the findings extrapolated to reflect the overall kernel execution time. The model focuses on obtaining a key metric describing the parallel process - a warp throughput, from which the total kernel execution time can be derived. Concurrency in this case is synonymous to occupancy through the number of active warps residing at an SM at a given time. Furthermore, an assumption is made that both the occupancy and the throughput are sustained for the entire time of the kernel's execution. An important part of the model are the bounds for warp throughput and latency, warp throughput is limited by throughput bound imposed by the hardware and its latency may not be lower than the one resulting from the instruction sequence comprising the kernel. Warp latency bound is obtained by direct inspection of the compiled kernel assembly code. Warp throughput bound is calculated based on requirements for resources available on the SM.

Quantitative methods, on the other hand, evaluate the characteristics and behaviour of a given kernel either by measuring or microbenchmarking kernel execution on actual hardware or by executing it, or parts of it, in a GPU simulator. These are often integrated into a compilation process of a kernel and are more automated than analytical ones. Examples include the following: an automated approach where the kernel is analysed to create a work flow graph [5] that focuses on measuring warp and instruction level parallelism, that is later parametrized to reflect execution process on a specific GPU unit; a microbenchmark based [53] method where instructions are extracted directly from the compiled source code of a kernel, backed by a functional simulator for memory accesses; Ocelot dynamic compilation framework [27, 28] leveraging PTX representation of a kernel, that is transformed into a form feasible to be modelled on a single CPU thread. Lastly, an interval analysis [24] technique, which is based on an assumption that by locating events causing performance degradation (memory stalls, cache misses, etc.), one can reason about the overall execution time of a kernel.

Statistical and machine learning methods $[25,52,31]$ are often implemented as separate frameworks that are highly automated and require additional training so that the neural network correctly recognises patterns and behaviour of the kernel code. The network learns the kernel's instructions composition given a set of input data and is then able to reason about the performance of subsequent kernel executions for different launch configurations. Consequently, they provide a complete simulation environment on their own and are not suitable for developing a new model that could be used within MERPSYS. Given the aim of this work is extending MERPSYS with a performance model for a GPU, we find them not relevant in our analysis.

3.2. GPU modelling in general-purpose simulators. General-purpose simulators are not tied to any specific device type or programming language and provide means to perform various kinds of simulation for many processing paradigms on parallel and distributed systems. Designing an application for such environments is not a trivial task as one needs to consider execution time, energy efficiency, reliability, maintainability and many other factors [48]. This is why most of the simulators a layer of abstraction over the actual hardware, what is a good thing when we consider a use case like modelling a complex grid system, but it may also be a significant drawback if one needs to model the parallel process on a specific hardware unit with a greater detail. All of further mentioned simulators implement the discrete-event simulation model [43], which means that the entire process of simulation is governed by events being processed and passed between entities. Such an event may represent a computation, communication, synchronisation or a resource access, with the details specified by the simulation environment itself. We have looked at the following tools with the focus on GPU simulation: GridSim [8], SimGrid [42, 21], CloudSim [9, 41, 47], GSSIM [29, 7], and MARS [20]. Table 3.1 summarises their suitability for this purpose, a general overview of their functionality was given in [15].

We have found that none of these simulators offers a convenient way to incorporate GPU modelling within it with the level of detail we require. This is mostly because their focus is on a different area of modelling, i.e. an HPC system as a whole, where computational units are described in terms of overall computational power. CloudSim with GPU extension [47] is closest to our requirements, but it still operates at the GPU computational task level and does not allow for precise modelling of kernels comprising the application. On the contrary, MERPSYS whilst being a general-purpose simulator itself, is a highly customizable solution that allows to drill down deep into internals of a single hardware unit, in our case a GPU. It allows easy modification 
TABLE 3.1

General-purpose simulators comparison

\begin{tabular}{|l|l|l|}
\hline Simulator & $\begin{array}{l}\text { Level of detail available for modelling a } \\
\text { hardware component }\end{array}$ & Suitability for GPU modelling \\
\hline GridSim & $\begin{array}{l}\text { Poor - computational power defined in terms } \\
\text { of MIPS or SPEC rating. }\end{array}$ & Not with the required level of detail. \\
\hline SimGrid & $\begin{array}{l}\text { High - complex analytical models may be } \\
\text { used. }\end{array}$ & $\begin{array}{l}\text { Theoretically yes, would require more } \\
\text { in-depth investigation of the possibility to } \\
\text { extend the existing framework with a custom } \\
\text { computational resource. }\end{array}$ \\
\hline CloudSim & High if extensions to the framework are used. & Yes, but not with the required level of detail. \\
\hline GSSIM & $\begin{array}{l}\text { Plugins that take many factors into account } \\
\text { may be written to describe the performance. }\end{array}$ & $\begin{array}{l}\text { Possibly, by writing plugins to define complex } \\
\text { application performance models. }\end{array}$ \\
\hline MARS & $\begin{array}{l}\text { Task modules execute logs containing MPI } \\
\text { call traces. There's no room for } \\
\text { customisation. }\end{array}$ & Not at all. \\
\hline
\end{tabular}

of the model by substitution of other hardware such as GPUs and repeating simulations, easy modification of formulas modelling execution and communication times, as well as relevant constants and coefficients.

3.3. Contributions of this work. Contributions of this work are as follows:

- We have incorporated the model proposed by Volkov [51] within the MERPSYS simulator [15], showing that it is well suited for practical implementations. We have used a simplified version of this model by reducing the scope of hardware units considered to CUDA cores, schedulers, and global memory system, for which we decided to use a trivial non-parametrized access model. For this version, we provide a complete set of equations (Eqs. 4.1 - 4.6) together with a detailed description of input parameters, creating an easy to grasp explanation of all the building blocks of the model.

- We have also extended the model with a scaling parameter, allowing to fine-tune it to better fit hardware and kernel characteristics. We propose Eq. 4.5 for calculating global memory bandwidth and introduce data transfer time calculation, whilst the original model considered only kernel execution time.

- We show that for the considered model configuration derived from results on one GPU of a given architecture can be directly applied to another GPU of the same architecture, introducing only the second GPU's specifications into the model. This makes the MERPSYS environment with the model deployed in it a suitable tool that is able to verify performance of a given application on potentially several cards without even having physical access to them.

4. Proposed Approach. Compared to other general-purpose simulators, MERPSYS is a much better fit for GPU modelling. It is mainly because of the very high level of detail available when modelling a hardware piece combined with a possibility to add custom functions describing device's behaviour. Hence, the computational devices are highly customizable which allows implementation of complex analytical models like the one being described in this section. Additionally, the hardware (computational) model may be customised to use additional data needed for GPU modelling. This data may be specified in the application model, which allows for easy parametrization of simulation runs. Another advantage is an extensible database of hardware components and ease of hardware model customisation, which allows for convenient evaluation of various setups. These can be activated just by selecting other GPUs, that have all the parameters in the database, through a graphical interface.

4.1. GPU Performance Model. To model the execution time of a single kernel on a single GPU we will rely on a model proposed by Volkov [51], we define Eq. 4.1 for this purpose. The number of warps launched is computed as shown in Eq. 4.2. For warp throughput, we use Eq. 4.3 (proposed by Volkov), the methodology for obtaining occupancy, latency bound, and throughput bound will follow. This model considers all of the important elements that contribute to the overall execution time of a kernel in most of the scenarios. Later on, 
we explain the meaning of each of these elements and the approach used to determine their values. Since the entire model is based on a concept of modelling a concurrent process in terms of a warp being executed on a Streaming Multiprocessor (SM) and the metrics are calculated for that single warp, it is required to extrapolate the findings to reflect the actual number of warps that were executed on the GPU and thus compute the result. For this purpose, Eq. 4.2 is used that takes as an input the launch configuration of a kernel i.e. the sizes of grid and block, and the result is the total number of warps that were launched to process the kernel.

$$
\begin{gathered}
T_{k}=\frac{W_{L}}{W_{T h} \times S M s \times S M_{\text {clock }} \times \lambda_{k}} \\
W_{L}=\text { grid size } \times\left\lceil\frac{\text { block size }}{\text { warp size }}\right\rceil \\
W_{T h} \approx \min \left(\frac{\text { occupancy }_{\text {Lat }}}{\text { bound }_{\text {bound }}}, T h_{\text {bou }}\right)
\end{gathered}
$$

where: $T_{k}$ - estimated kernel execution time $[\mathrm{s}] ; W_{L}$ - number of warps launched; $W_{T h}-$ processing rate of warps [1 / cycle]; $S M s$ - number of streaming multiprocessors; $S M_{\text {clock }}-$ SM core clock [cycles / s]; $\lambda_{k}-$ scaling parameter; grid size - number of blocks in a grid; block size - number of threads in a block; warp size - 32

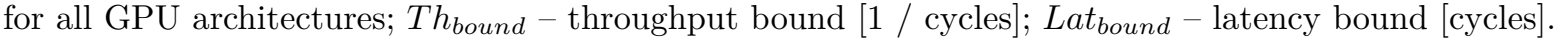

$S M s$ and $S M_{\text {clock }}$ parameters are needed to extrapolate the model from a single warp in scope of a single multiprocessor to one that fits the processing model of the GPU. These two parameters, when multiplied, yield the processing power of the entire device in cycles per second. The $\lambda_{k}$ value is a ratio between estimated and measured execution times that adjusts the model to fit the characteristics of a given kernel launched on a specific device architecture. It shall be obtained experimentally by measuring the execution time of a real application combined with running a simulation with an initial model. Once calculated the new $\lambda_{k}$ can be used to perform simulation across varying data sizes and different GPUs. It was experimentally proven by the authors of another model $[2,3]$ that a single $\lambda_{k}$ value is sufficient for accurate simulations in scope of a single device architecture. When used for a different architecture the accuracy of the model drops and it is advised that $\lambda_{k}$ is recalculated. To calculate the $\lambda_{k}$ we divide the estimated execution time, by the measured one: $\lambda_{k}=\frac{T_{e s t i m a t e d}}{T_{\text {measured }}}$.

Calculation of warp throughput, the most essential part of the model, as given by Eq. 4.3, is the most challenging task. Three parameters need to be extracted from the kernel code in close consideration of a specific GPU architecture that one intends to model. These parameters are warp latency and throughput bounds, and the achieved occupancy, we will describe them in detail in subsequent sections.

4.1.1. Occupancy. The first method of retrieving the parameters needed for occupancy calculation is to compile the code with verbose ptxas output, this is done by passing '-Xptxas -v' command line option to nvcc [38]. A sample result is shown in Listing 1. It tells us that the kernel uses 8 registers per thread and does not use any shared memory, otherwise information about the amount of shared memory used would be included in the output. cmem stands for constant memory and does not concern our analysis. Having determined the number of registers and amount of shared memory required, we enter these together with threads per block count (block size) into the NVIDIA occupancy calculator [34] to get the theoretical occupancy. Another way is to profile the application, NVIDIA Visual Profiler not only displays the values of shared memory and registers used but it is even capable of calculating both the theoretical and achieved occupancies.

LISTING 1

Verbose ptxas output for saxpy2 kernel

ptxas info: Function properties for saxpy 2

Used 8 registers, 344 bytes cmem[0] 
4.1.2. Latency bound. We construct an execution graph of the kernel with nodes representing instructions and edges representing latencies as shown in Volkov's work [51] and then apply a critical path method to it to find the latency. Critical path is the latency bound only if we assume that all of the warps are the same - they execute the very same instructions, so this approach will not work if there is a substantial control flow divergence in a kernel. In such a case, a different set of instructions resulting from different branches being executed should be considered.

The graph considers latencies of the following kind: register dependencies and thus instruction execution latency, instruction issue latency, and memory stalls to describe how the instructions comprising the kernel are depend between themselves. This approach is valid because there is no out-of-order execution present in the GPUs, meaning that the sequencing of the instructions is maintained in a strict order. It is also more precise than when making an assumption that a kernel is a simple sequence of dependent instructions as in [2, 11, 40] and simply summing the latencies of all instructions comprising the sequence, treating the result as a total cost in clock cycles of executing this kernel by a single warp. The latter approaches fall short because there are independent functional units within the SM, which results in latency hiding, e.g. in case of waiting for memory accesses. Furthermore, even in the case of a single functional unit type the instructions are processed in a pipelined-based manner and thus ILP (Instruction Level Parallelism) exists.

Volkov reports [51] that there are two other types of latency, the latency between two independent instructions from a single warp, called ILP latency and latency resulting from a replacement of a terminated thread block, we address both of these. What is more, depending on the architecture, multiple warp schedulers per SM may be present and two instructions per warp may be issued every instruction issue time if mutually independent instructions are available for execution. The effect of dual issue is considered as well, in which case the ILP latency is expressed as 0 cycles. We omit double precision and special function units, both of which have their own pipelines with different latencies for various instructions. Moreover, our approach to modelling global memory accesses is simplified as we do not consider gradual saturation effect [51], which manifests itself in a form of increasing memory access latency as the number of memory transaction increases and memory bus gets saturated. It should also be noted that the modelling of shared memory accesses was omitted. These omissions do not affect results of our work as we have deliberately chosen a simple kernel, which does not make use of aforementioned functional units. Furthermore, the model is designed in such a way that it may easily be extended with these elements in the future.

4.1.3. Throughput bound. To obtain the throughput bound, each of the hardware resources available on the SM must be considered separately, including: CUDA cores, Special Function Units (SFUs), double precision units, schedulers, and global and shared memories. We will narrow our analysis to three of them as denoted by Eq. 4.4. The tightest bound, that is the highest number of cycles required to execute the warp's instructions due to a limited processing power of the functional units, is selected as the limiter of the performance and then a reciprocal is calculated to obtain the bound represented in warps per cycle.

$$
\begin{gathered}
\left.T h_{\text {bound }} \approx \frac{1}{\max \left(\frac{W_{s z} \times I N S_{C U D A}}{\text { CUDA cores }}, \frac{I N S_{\text {issued }}}{\text { schedulers }}, \frac{\text { GMem } m_{\text {bytes }}}{\text { GMem }}\right)}\right) \\
\text { GMem }_{b w} \approx \frac{G M e m_{\text {clock }} \times \frac{\text { bus width }}{8} \times \text { data rate }}{S M s \times S M_{\text {clock }}}
\end{gathered}
$$

where: $W_{s z}$ - warp size; $I N S_{c u d a}$ - number of instructions to be executed by CUDA cores; INS $S_{\text {issued }}-$ total number of instructions issued (including dual-issues and re-issues); $C U D A$ cores - number of CUDA cores available on the SM; schedulers - number of schedulers available on the SM; GMem bytes $_{\text {- number of bytes }}$ transferred with global memory for each warp [bytes]; GMem $_{b w}$ - peak throughput of the memory system

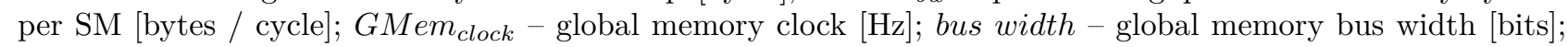
data rate - data rate multiplier depending on the kind of memory used.

4.2. Obtaining hardware parameters. Each of the instructions issued by a thread is assumed to require a well-known number of clock cycles to complete, this is a simplified approach not considering varying memory 
TABLE 4.1

Instructions latencies for Maxwell architecture

\begin{tabular}{|l|l|}
\hline Operation & Latency \\
\hline add, sub (integer, 32-bit FP) & 6 \\
\hline mad (32-bit FP) & 6 \\
\hline shl, shr & 6 \\
\hline mov, setp & 6 \\
\hline bra (taken) & 12 \\
\hline bra (not taken) & 10 \\
\hline ILP latency & 3 \\
\hline Terminated block replacement & 150 \\
\hline Global memory access & 350 \\
\hline
\end{tabular}

access times, varying instruction latency depending on the instruction sequence and other effects. Based on papers [4, 51] and additional reasoning, Table 4.1 presents latencies we assumed in this work. For latencies of Tesla, Fermi and Kepler architectures see [4], for Pascal and Volta see [26]. For a detailed information regarding meaning of the mnemonics refer to [36].

There are two sets of parameters in Eq. 4.4. The first one is related directly to the hardware characteristics of a given device, it includes: warp size, number of CUDA cores and warp schedulers, and the throughput of the global memory. At the time of writing the warp size is a constant value of 32 for all GPU architectures. The number of CUDA cores and warp schedulers may be obtained from GPU hardware specification. Theoretical bandwidth of the GPU's global memory available per single SM every cycle is given by Eq. 4.5, for which all the parameters are available in hardware specification except the data rate that is derived from memory type and equals 2 or 4 respectively for GDDR3 and GDDR5 [33]. Although the peak bandwidth is not sustainable in practice for the entire execution of a kernel because the memory clock may drop due to dynamic frequency scaling and furthermore would require an ideal access pattern and sufficient occupancy, a value close to this theoretical limit is attainable [51]. Considering this fact, we use this bandwidth in the proposed model. What is more, we do not differentiate between loads and stores and assume them to behave in the same way. Additionally, we limit the model to a scenario where there are no cache hits and the accesses are fully coalesced. If we were to include the possibility of cached accesses in the model then we would need to consider the fact that depending on the device architecture accesses to global memory are cached in L1 and L2 caches, hence the number of cache hits should be subtracted from the actual number of DRAM accesses. The ratio of cache hits could be obtained by launching the application in a profiler [11].

The second set of parameters: $I N S_{C U D A}, I N S_{\text {issued }}$ and $G M e m_{\text {bytes }}$ describes application execution characteristics, all three are extracted directly from compiled SASS code of the kernel, description of these follows. To calculate the number of CUDA core instructions we count all occurrences of operations that are executed using these cores: FP32, INT32, logical, etc. Getting a sum of all instructions that were issued is a little more demanding because dual-issues and re-issues of instructions need to be accounted for. In the former case, two instructions are issued in a single issue cycle and hence are counted as one. In the latter, a single instruction must be counted multiple times depending on the number of re-issues. A re-issue typically occurs when there are bank conflicts when accessing shared memory or when an access to global memory is uncoalesced and must be split into several separate transactions. This also affects number of bytes transferred per warp, for example when fetching a single 32-bit value per thread, a stride of two would result in 256 bytes transferred instead of 128 and a single additional re-issue of memory transaction instruction, stride of 4 would cause 3 additional re-issues and increase the number of bytes to be transferred to 512. Numbers of functional units and clock values for the devices as listed in Section 6.1 were obtained from vendor's documentation and using ndivia-smi [35].

4.3. Modelling communication time. Performing computations on a GPU requires the data to be transferred to the device prior to launching a kernel and once its execution completes the results must be fetched back to the main operating memory. Memory transfer between the host and the device may be considered an instance of a point-to-point communication, for which the communication time is given by the following equation 
[12]:

$$
T_{c}=t_{s}+\frac{n}{b w \times \lambda_{c}}
$$

where: $T_{c}$ - data transfer time; $t_{s}$ - startup time; $n$ - data size; $b w$ - bandwidth; $\lambda_{c}$ - scaling parameter.

Startup time is the time needed to initiate data transfer that depends on latency and runtime overhead, it can be measured by sending a very small data packet. Bandwidth is dependent on the configuration of the PCIe bus that connects the devices, for example a theoretical bandwidth of PCIe v3.1 $16 x$ bus in single direction is $16 \mathrm{~GB} / \mathrm{s}$ or $15.8 \mathrm{~GB} / \mathrm{s}$ if we consider $128 \mathrm{~b} / 130 \mathrm{~b}$ encoding. Furthermore, we have noticed that the bus is better utilised for larger data sizes but even for the largest transfer of 2GB the throughput was noticeably lower than the theoretically achievable one. Due to this fact, in our implementation we introduce an additional scaling parameter $\lambda_{c}$ to Eq. 4.6 that allows us to fine-tune the link bandwidth so that it reflects the actual characteristic of a given hardware setup.

Note that both the $\lambda_{c}$ and $t_{s}$ will have different values for each transfer direction. The scaling parameter can be obtained similarly to the one for the kernel execution time $\left(\lambda_{k}\right)$, i.e. by dividing the estimated transfer time by the measured one. To get the value of the bandwidth parameter we first need to determine the PCIe configuration, for this purpose we have used nvidia-smi. Having obtained these two values, we can refer to hardware documentation to get the bandwidth parameter.

5. Implementation. It is easiest to present how the performance model is constructed by example. For this purpose we use saxpy2 kernel (Listing 2) that is a slightly modified version of a kernel from the blog post [22]. The main difference when compared to a regular saxpy is that instead of a single multiplication an equivalent number of additions is performed, this allows us to benchmark kernels of various compute intensities. The highlighted part of the code is responsible for a compute intensity of the kernel, i.e. the number of arithmetic instructions executed by each warp. Input data size is determined by the number of elements in the vectors as: number_of_elements_per_vector $\times 2 \times 4$ Bytes.

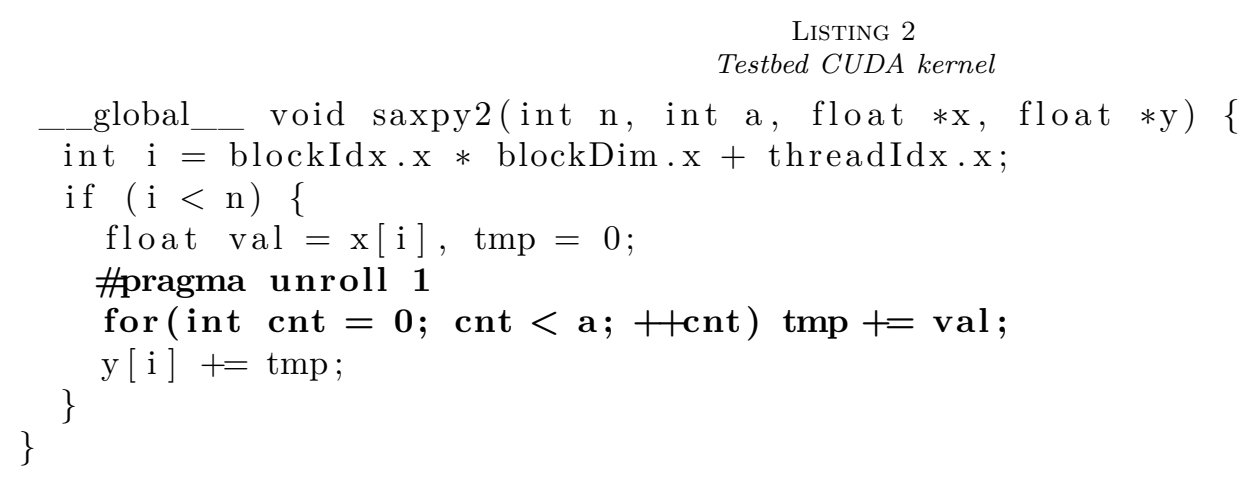

To accurately determine the kernel's instruction composition, we cannot simply analyse the $\mathrm{C}$ or $\mathrm{C}++$ source code since we do not know the optimisations that took place and thus the actual instructions that were generated by the compiler. For this purpose we need to analyse SASS code (see Section 2.1.1), to extract it from a compiled binary file we use cuobjdump [36]. Listing 3 is a SASS representation of the saxpy2 kernel that was compiled for $s m \_$52, for the sake of brevity we skip the irrelevant NOP instructions from the very end. The highlighted part represents the loop governing the kernel's arithmetic intensity.

LisTING 3

SASS representation of the saxpy2 kernel

MOV R1, c $[0 \times 0][0 \times 20]$;

S2R R0, SR_CTAID.X;

S2R R2, SR_TID.X;

XMAD.MRG R3, R0.reuse , c [0 x0] [0 x8].H1, RZ;

$\mathrm{XMAD} R 2, \mathrm{R} 0$.reuse, $\mathrm{c}[0 \times 0][0 \times 8], \mathrm{R} 2$;

XMAD. PSL.CBCC R0, R0.H1, R3.H1, R2; 


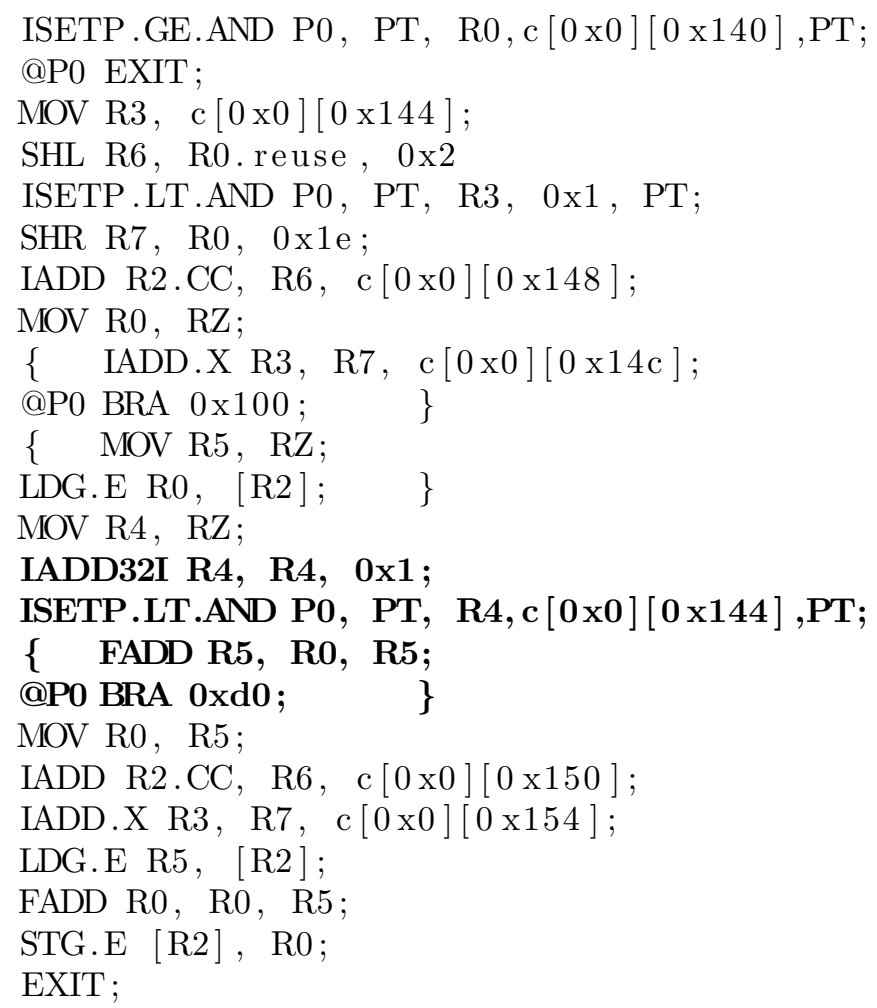

5.1. Creating kernel execution graph. To construct an execution graph of saxpy2 kernel we start off by creating a directed graph representing the sequence of instructions comprising the kernel based on Listing 3 . Each instruction is a single node, additional start and end nodes mark respectively the entry and exit point of the kernel. Edges represent the dependencies between the instructions and are labelled with latency values from Table 4.1. Straight, solid arrows are used for the ILP latency, which equals 3 cycles for Maxwell architecture. There are total of 3 dual-issues, these edges are labelled with 0 . The next step is to include latencies from register dependency, these are shown as dotted arrows. We do this by reading the SASS line by line and verifying whether any of the input registers of the current instruction has its value written to by one of the preceding instructions, if so then a register dependency exists and we add a new edge connecting the predecessor with successor labelled with latency value of the preceding instruction. If this instruction is a memory access, then we use the latency of the storage area being referenced, which in case of this kernel is always the global memory as cache hit ratio equals $0 \%$. This is true because there is no re-use of data - each element is accessed by a single thread and exactly once. We verified correctness of this assumption by inspecting the profiler output for this kernel regarding the memory throughputs. Lastly, we label the inbound edge of the virtual end node with a terminated thread block replacement latency.

Once the graph is complete and labelled we use the critical path method to find the latency bound of the kernel. Figure 5.1 shows the resulting graph with critical path highlighted in blue. The part of the graph where nodes are ellipses is the computational loop, which may execute multiple times depending on the compute intensity parameter passed to the kernel. When calculating the latency bound, the critical path of the loop needs to be multiplied by the number of loop iterations. The final equation for latency bound is as follows: latency bound $=92+700+150+24 a=942+24 a$ where the first term is sum of instructions' latencies, the second one is latency of memory accesses, the third is thread block replacement latency, and the fourth one is latency of the loop multiplied by the compute intensity parameter 'a'. We have created and analysed the graph manually, but creation of an automated tool is entirely possible.

5.2. Obtaining throughput limits. As the first step in throughput limit calculation for the saxpy2 kernel we need to count instructions in the generated SASS. It can be done either by reading directly from 


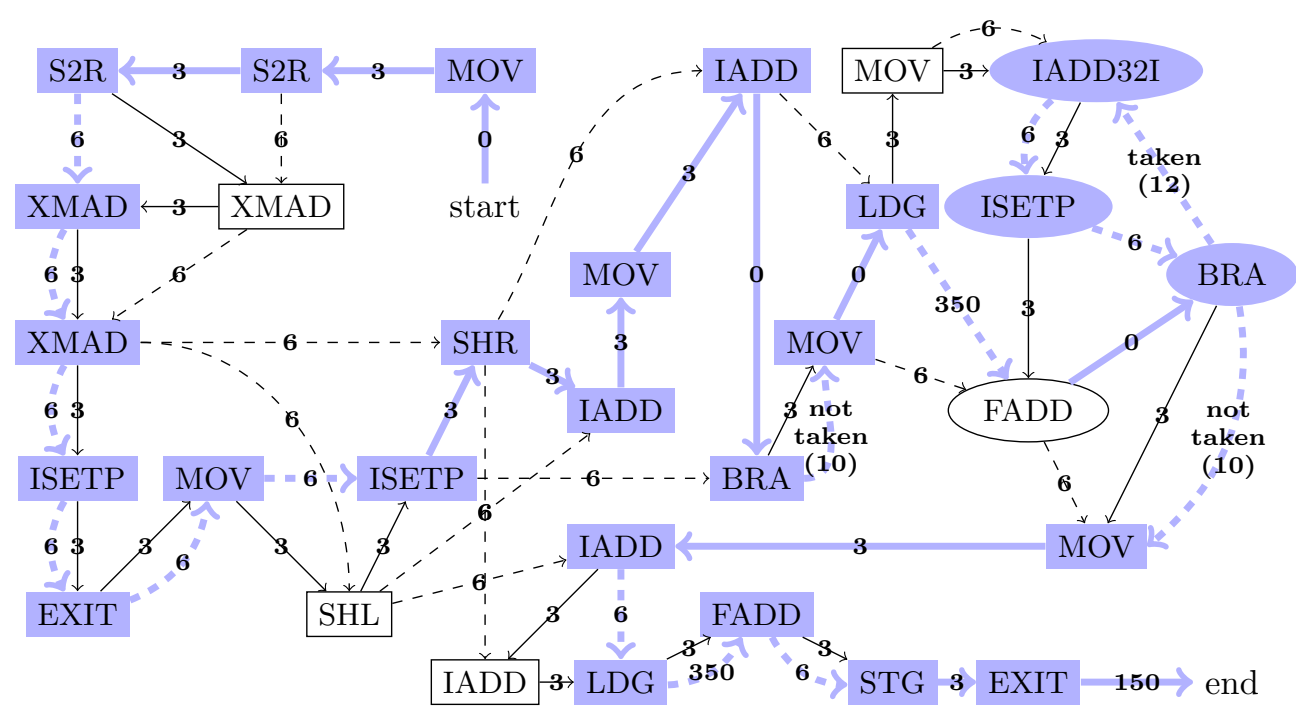

FIG. 5.1. Execution graph for saxpy2 kernel

the assembly listing (Listing 3) or based on the kernel execution graph (Fig. 5.1). In our example, we have 23 CUDA core instructions that are executed once and 4 instructions in a loop, so the resulting equation is: $I N S_{C U D A}=23+4 a$. It should be noted that in this kernel everything except memory accesses is processed by CUDA cores, this would not be the case if there were any instructions designated for a double precision or SFU units. When getting the number of instructions issued we must remember to address the dual-issues and re-issues. In our kernel, we have 3 accesses to global memory, all three are fully coalesced so there are no re-issues taking place. There are 3 dual-issues, each resulting in a pair of instructions being issued together during a single cycle.

Summing up, we have 23 CUDA core instructions issued once and four of these in a loop, three memory instructions, and three dual-issues which we subtract from the total number, so we get: $I N S_{\text {issued }}=23+3-3+$ $4 a=23+4 a$. The number of bytes transferred to and from a global memory depends on four factors: the number of the memory access instructions, cache hit rate, operand size, and access pattern. The saxpy2 kernel contains 3 memory access instructions: two loads and a single store, but for the sake of brevity we do not differentiate between these two as the difference is marginal. In all three cases, a single 4-byte value is accessed per thread and as was mentioned in preceding paragraph, the accesses are fully coalesced so there are no redundant memory transactions when accessing the data, this gives us: MMem $_{\text {bytes }}=3 \times 4$ bytes $\times 32=384$ bytes. Furthermore, there are no cache hits hence all these bytes are accessed directly in the global memory and we can entirely omit both the L2 and L1 caches. If this was not the case then we would separately consider a fraction of $G M$ embytes $_{\text {b }}$ equal to cache hit ratio as being accessed from cache, which has a higher throughput than the global memory.

Now that we have all the throughput related parameters extracted from the kernel we need to list those specific to the device the application will run on, which is NVIDIA GeForce GTX 970 in our case. The first two parameters can be obtained directly from card specifications shown in Section 6.1, compute capability of our card is 5.2, hence we have: $C U D A$ cores $=128$, schedulers $=4$. The third parameter - bandwidth of the global memory can be calculated using Eq. 4.5 substituting the required values as: $G M e m_{\text {clock }}=1753 M H z$, bus width $=256$ bits, data rate $=4, S M s=13, S M_{\text {clock }}=1253 \mathrm{MHz}$. This gives us: GMem $_{\text {bw }}=13.78 \frac{\text { Bytes }}{\text { cycle }}$ The final step is to gather up all these parameters and substitute them to Eq. 4.4:

$$
T h_{\text {bound }} \approx \frac{1}{\max \left(\frac{32 \times(23+4 a)}{128}, \frac{23+4 a}{4}, \frac{384}{13.78}\right)}
$$

5.3. Equations for communication time. Our testbed with GTX 970 uses PCIe v3.1 16x. Based on this fact and our measurements we substitute the parameters of Eq. 4.6 and thus we get the equation for data 


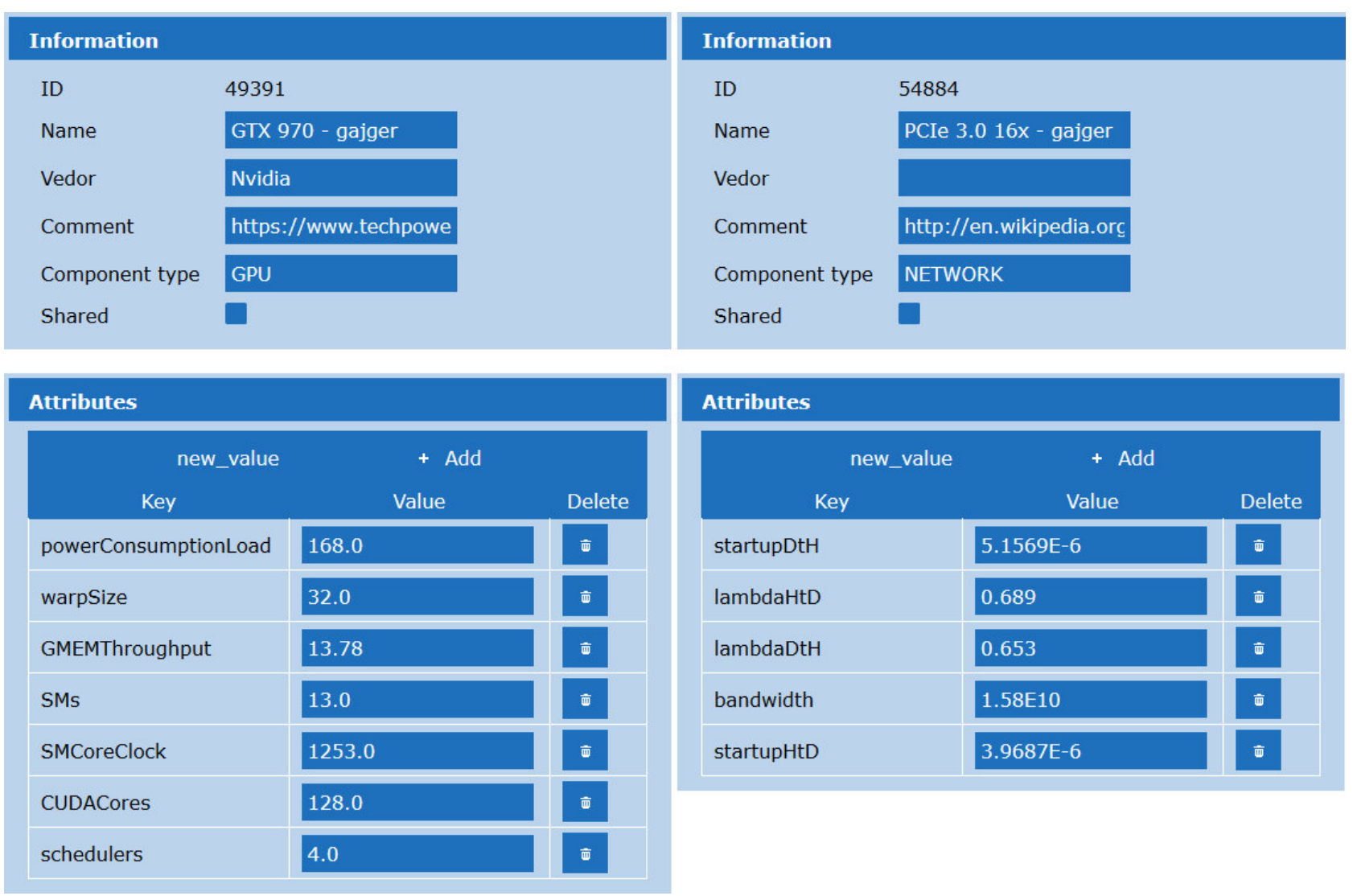

FIG. 5.2. Hardware units with customised attributes: GPU (left), interconnect (right)

transfer time in function of its size for both directions as shown below.

$$
T_{\text {Host to Device }}=3.9687 \times 10^{-6}+\frac{n}{15.8 \times 10^{9} \times 0.689} \text { and } T_{\text {Device to Host }}=5.1569 \times 10^{-6}+\frac{n}{15.8 \times 10^{9} \times 0.653} \text {. }
$$

5.4. Incorporating the model within MERPSYS. In this section, we show how the theoretical model was implemented in MERPSYS. At first, we demonstrate how to create a system model representing our testbed, including the creation of new hardware units. Then we proceed to computational model definition, i.e. the programmatic representation of what was shown in previous sections. Next, we move to the application model definition, which will represent the CUDA application being modelled, explaining how all these pieces are tied together and finally elaborate on how to customise the application launch parameters and execute the simulation. Creation of hardware units that are customised with parameters required by our theoretical model was the first implementation step. All previous measurements were performed using GTX 970 and PCIe v3.1 16x. For these two devices we have created a representation in MERPSYS' database as shown in Fig. 5.2. It should be further noted that the $\lambda_{c}$ parameter from Eq. 4.6 was included as an attribute of the PCIe hardware unit. We assumed that the effective bus utilisation is a parameter of the hardware itself and its value does not change for different application types if the memory transfers are performed using the same functions. Shall a requirement for this parameter be customizable via application model arise, it can be moved there and passed in the function calls the same way it was done for kernel parameters, as shown in Section 5.4.2.

The next step to perform is creation of a testbed representation using the hardware model editor in MERPSYS editor application. Two hardware units that we have just created are used in this model together with a unit representing the CPU. Note that this model directly resembles the actual hardware structure where GPU and CPU are connected using a PCIe bus. 


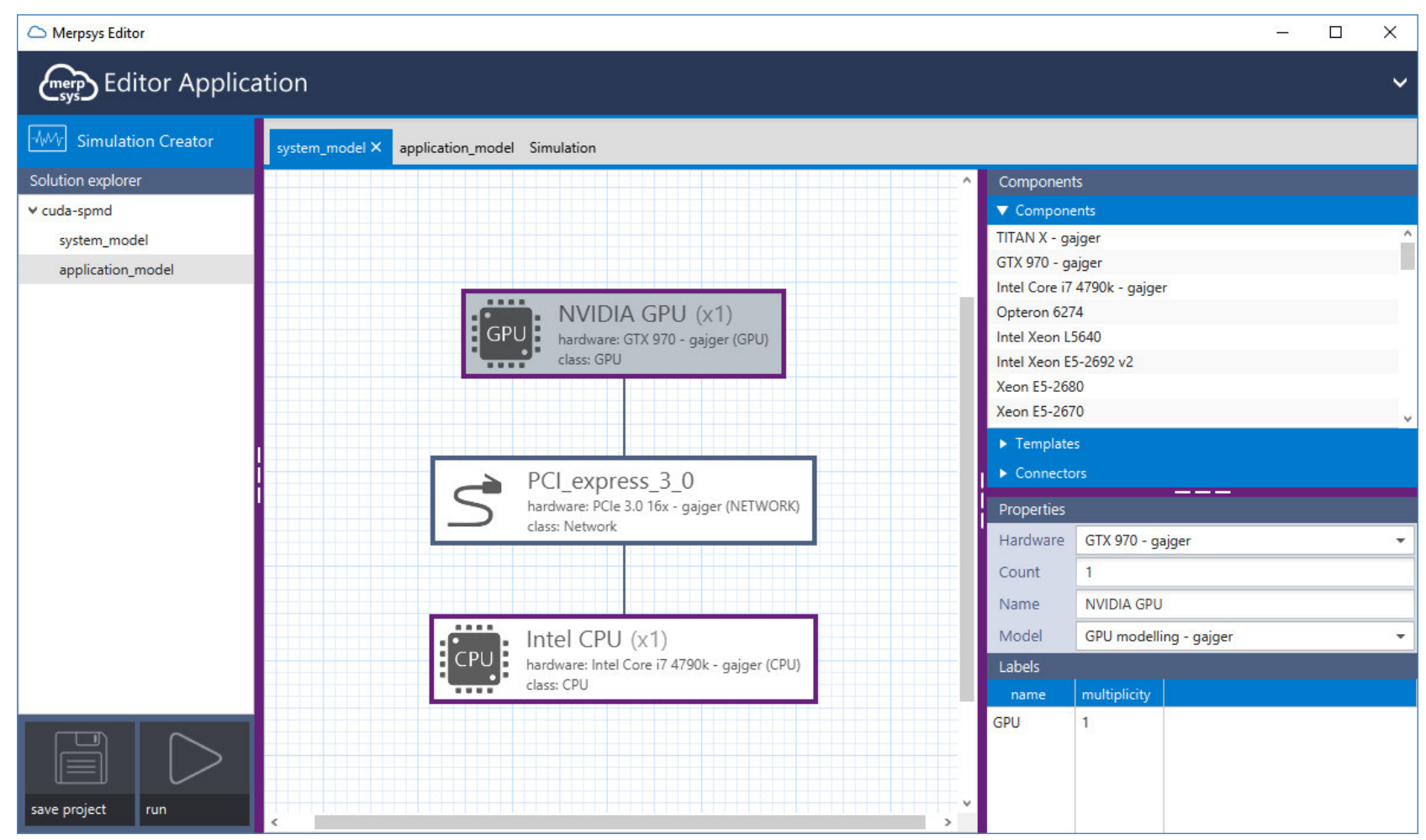

FIG. 5.3. Testbed configuration in MERPSYS system model editor

Figure 5.3 is a screenshot from the editor application showing the created model, GPU unit is selected with its properties visible on the right side, moving from the top to the bottom:

1. Hardware - hardware unit represented by this element, in our case the GTX $970 G P U$ visible on the left of Fig. 5.2;

2. Count - the number of hardware units of a given type, for example in a multi-GPU setup we could set the count to $2,3,4$, etc.;

3. Name - displayed name of the element;

4. Model - computational model connected to the element, in our case the one from Listing 4, description of which will follow;

5. Labels - labels assigned to the element, these form the connection between hardware and application models. Name is the identifier of the label and multiplicity denotes the number of processes with this label that may be executed on a single unit of this type.

5.4.1. Computational model. Another crucial component of the simulation suite is the computational model, in which we have implemented the equations comprising the model. These are written in JavaScript using MERPSYS' so-called shallow functions, one of them (an implementation of Eq. 4.1) is depicted in Listing 4. Others are not presented for the sake of brevity, but their implementation is similar. Parameters characterising hardware, i.e. hardware unit attributes are directly accessible from within the computational model functions. For example: SMCoreClock or SMs which reference attributes of the GPU (the ones from Fig. 5.2). On the other hand, application specific parameters are passed from the application model using proper arguments of the computation or communication function calls. These are then accessible as JSON objects containing key-value pairs. When we look at the signature of getTimeGPUModel() we can notice params argument, which is this JSON object. The value stored under a specific key is accessed using params.get() method. 


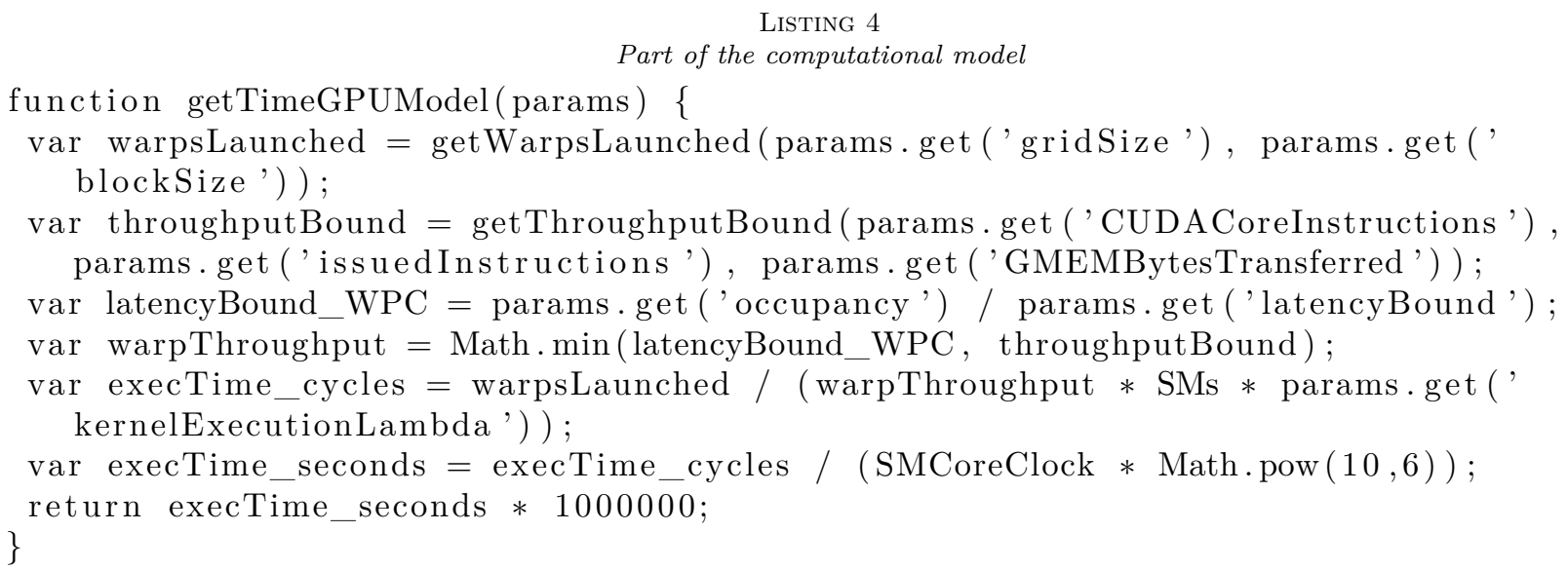

5.4.2. Application model. The application model is an abstract representation of the program being modelled, in our case it consists of few data transfers and a kernel launch, the model is depicted in Listing 5 and the source code for the host part of the CUDA application is given in Listing 6 . At the very beginning parameters characterising the kernel are defined, this part is based on the idea described in the section concerning extraction of kernel parameters. Next we have the actual application divided into two branches that are distinguished based on labels (types of processes), one for a CPU and one for a GPU. It is visible that the CPU only handles data transfers and each of the communication functions specified for it has its counterpart present in the GPU section. Sequencing of the operations that originate from the communication functions calls is managed internally in MERPSYS. Also note the artificial synchronisation point (marked with a comment in the source code), send in MERPSYS is non-blocking whilst cudaMemcpy() is blocking, hence the next transfer can only be performed when the preceding one is complete. The input parameters of the theoretical model for the kernel execution time are specified in the GPU section, these are put into a key-value map, that is converted by MERPSYS into a JSON object and passed to the appropriate function of the computational model, in our case it is getTimeGPUModel(). For the sake of brevity we have omitted most of the parameters and left only CUDACoreInstructions as an example, the others are set similarly.

LISTING 5

Application model

Integer CUDACoreInstr $=$ new Integer $(23+4 *$ computeIntensity $)$;

Integer issuedInstructions $=$ new Integer $(26+4 *$ computeIntensity $)$;

Integer GMEMBytesTransferred = new Integer (384);

Integer latencyBound $=$ new Integer $(942+24 *$ computeIntensity $)$;

Integer gridSize = new Integer $((\mathrm{N}+$ blockSz -1$) /$ blockSz $)$;

Double kernelExecutionLambda = new Double $(0.703787)$;

if (tag . equals ("CPU")) \{

sim .p2pCommunicationSend (4*(double)N, "GPU" , ConstVar. HostToDevice) ;

// artificial synchronisation point

sim . p2pCommunication Receive ("GPU") ;

sim.p2pCommunicationSend ( $4 *($ double $)$ N, "GPU" , ConstVar. HostToDevice) ;

sim . p2pCommunicationReceive ( "GPU") ;

\} else \{

sim . p2pCommunicationReceive ( "CPU") ;

// artificial synchronisation point

sim.p2pCommunicationSend (0, "CPU") ;

sim . p2pCommunicationReceive ( "CPU") ;

Map GPUModelParams = new HashMap ( ) ;

GPUModelParams .put (" CUDACoreInstructions " , CUDACoreInstr) ; 


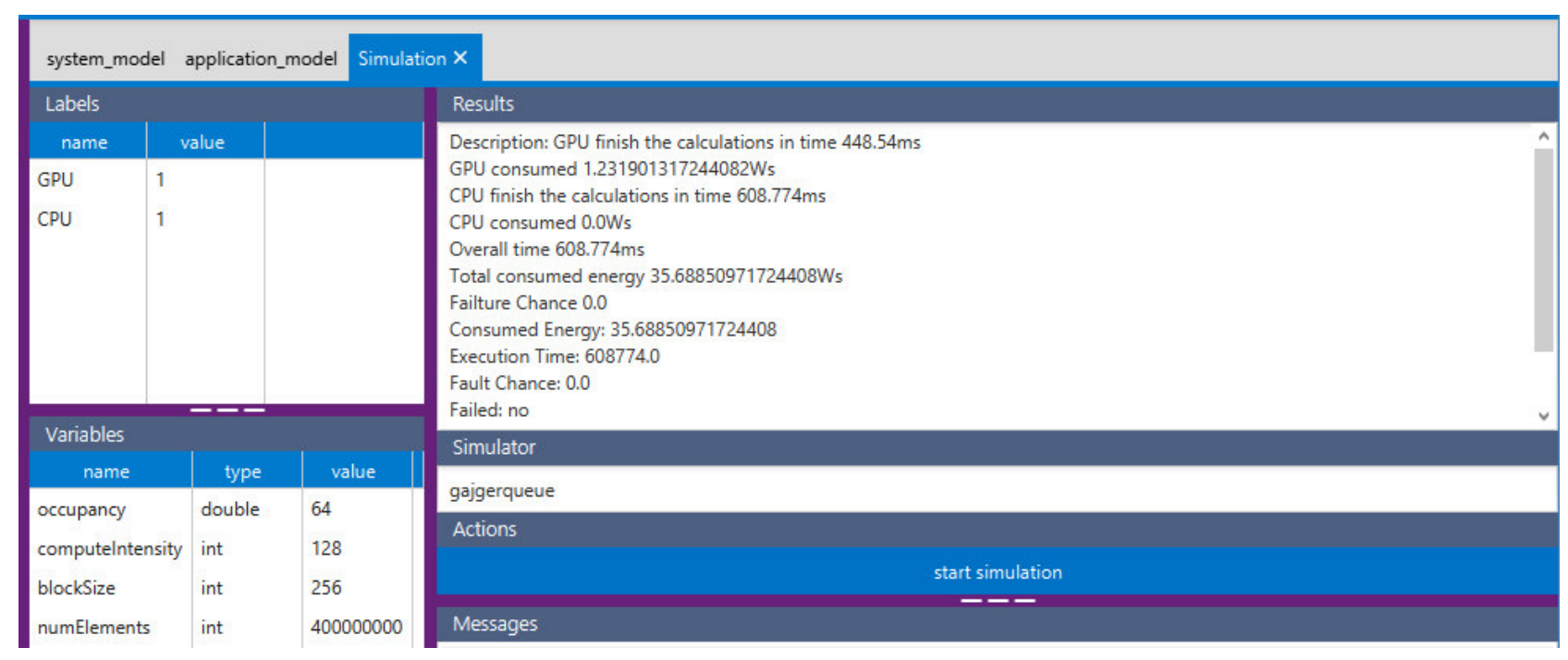

FIG. 5.4. Simulation launch screen

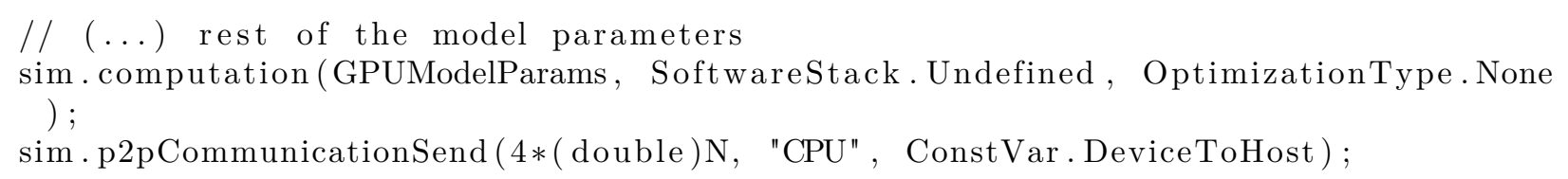

Most of the parameters defined in the application model are not constant values, but are instead computed based on the model input parameters (e.g. occupancy), that are specified in the Variables section of the editor application, which may be customised either from the application model editor screen or simulation launch screen, the latter is shown in Fig. 5.4. The Variables section is in the bottom left corner, values located there may be easily changed between simulation runs which allows for an effortless customisation. For example, if we want to test the application's behaviour for a different number of input elements or change the characteristics of the kernel by tweaking computeIntensity parameter. In the Labels section of the simulation screen, we define what processes are going to be launched. A label represents a process or a thread and its value is the number of processes or threads of this type, note that these are not the same labels that we have already seen in the hardware model, although both kinds are directly related. The relation is that a process identified by label $G P U$ requires the very same label to be assigned to at least one of the hardware components.

Furthermore, the maximum number of processes or threads of a given type that may be launched depends on the hardware units' execution capacity, which we may compute by inspecting the hardware model and multiplying the label multiplicity by the count of hardware units. For example, in Fig. 5.3 label GPU has multiplicity of 1 and there is only a single unit, hence when launching the simulation, the number of processes of this type must not exceed 1. This label is also referenced in the application model using tag.equals() construction, this demonstrates that the labels are what binds all of the simulation pieces together. With the variables and labels defined, a simulation can be launched. We first specify the name of a simulation queue, gajgerqueue in our case, this name must match the one that was used when a simulator application was started. After specifying a proper name of the simulation queue, we start the simulation, and once it finishes its execution the outcome is presented in the top right corner in the Results section. The simulated application execution time is listed as the Overall time in simulation results (Fig. 5.4), we will refer to it in the next section when comparing predicted values with the ones measured from the actual runs.

6. Experiments and Results. We have performed numerous tests for various configurations to verify accuracy of the implemented solution in four scenarios where different parameters change. In the first case, all parameters but the compute intensity were constant, the second one included modification of the block size in 
TABLE 6.1

Testbed GPUs

\begin{tabular}{|c|c|c|c|}
\hline & GTX970 & TITAN X & GTX1070 \\
\hline SMs & 13 & 24 & 15 \\
\hline SM clock $[\mathrm{MHz}]$ & 1253 & 1076 & 1923 \\
\hline Compute capability & \multicolumn{2}{|c|}{5.2} & 6.1 \\
\hline Schedulers per SM & \multicolumn{3}{|c|}{4} \\
\hline CUDA cores per SM & \multicolumn{3}{|c|}{128} \\
\hline Memory clock $[\mathrm{MHz}]$ & \multicolumn{2}{|c|}{1753} & 2002 \\
\hline Bus width [bits] & 256 & 384 & 256 \\
\hline Data rate & \multicolumn{3}{|c|}{4 (GDDR5) } \\
\hline Global memory throughput per SM [bytes / cycle] & 13.78 & 13.03 & 8.88 \\
\hline Kernel execution $\lambda_{k}$ & \multicolumn{3}{|c|}{0.703787} \\
\hline
\end{tabular}

TABLE 6.2

PCIe bus characteristics for testbeds

\begin{tabular}{|l|l|l|l|}
\hline Bus & v3.1 x16 & v2.0 x4 & v3.1 x16 \\
\hline OS & Ubuntu 14 & Ubuntu 16 & Windows 10 \\
\hline Driver ver. & 352.21 & 384.111 & 388.19 \\
\hline$t_{s} \mathrm{HtD}[\mathrm{ms}]$ & 0.00397 & 0.00733 & 0.0244 \\
\hline$t_{s} \mathrm{DtH}[\mathrm{ms}]$ & 0.00516 & 0.01168 & 0.0283 \\
\hline$\lambda_{c} \mathrm{HtD}$ & 0.689 & 0.844 & 0.452 \\
\hline$\lambda_{c} \mathrm{DtH}$ & 0.653 & 0.842 & 0.447 \\
\hline$b w[\mathrm{~GB} / \mathrm{s}]$ & 15.8 & 2 & 15.8 \\
\hline
\end{tabular}

addition to compute intensity, the third scenario concerned varying occupancy and the fourth changing input data size. In the first three scenarios, we measured only the kernel execution time as the data size was constant and memory copying could be omitted. In the last one, however, we included the measurements of the data transfer times in both directions, as well as the execution time of the entire application. All measurements in this section were repeated 10 times and then a mean value was used.

6.1. Testbed Environment. The tests were performed using three testbeds, one with GTX 970 and PCIe v3.1 16x, the second with GTX TITAN X (Maxwell edition) and PCIe v2.0 4x, and the last one with GTX 1070 and PCIe v3.1 16x. The first two were running Linux, and the last one Windows (see Table 6.2). The model was calibrated for the setup with GTX 970 and the kernel execution $\lambda_{k}$ was found to equal 0.703787 , it also turned out that the value of $\lambda_{k}$ did not change for the second GPU. This is expected behaviour since both devices are of the same architecture (compute capability), which proves the correctness of the model. We have also used the same $\lambda_{k}$ for the third GPU, representing a newer architecture (Pascal). The results were still accurate, it is likely because both architectures do not differ much in terms of instruction types and latencies. The devices differ in number of SMs, SM clock and in terms of memory system, with GTX TITAN X having wider memory bus, the differences and similarities are summarised in Table 6.1.

For interconnects we have measured data transfer startup time $\left(t_{s}\right)$ and PCIe bus utilisation $\left(\lambda_{c}\right)$ separately for each transfer direction: host to device (HtD) and device to host (DtH), the results are shown in Table 6.2. The transfer is slightly more efficient in the former case. We have also observed that bus utilisation was lower on Windows, when compared to Linux. This was likely caused by additional overhead incurred by Windows Display Driver Model (WDDM) [10].

6.2. Testbed Application. We have prepared a sample CUDA application that allocates the data, copies it from host to the device, calls saxpy2 kernel (Listing 2) and then fetches the results back into the host memory. The code of the application used for tests is given in Listing 6 and its representation in MERPSYS application model was already presented in the previous section (Listing 5). 


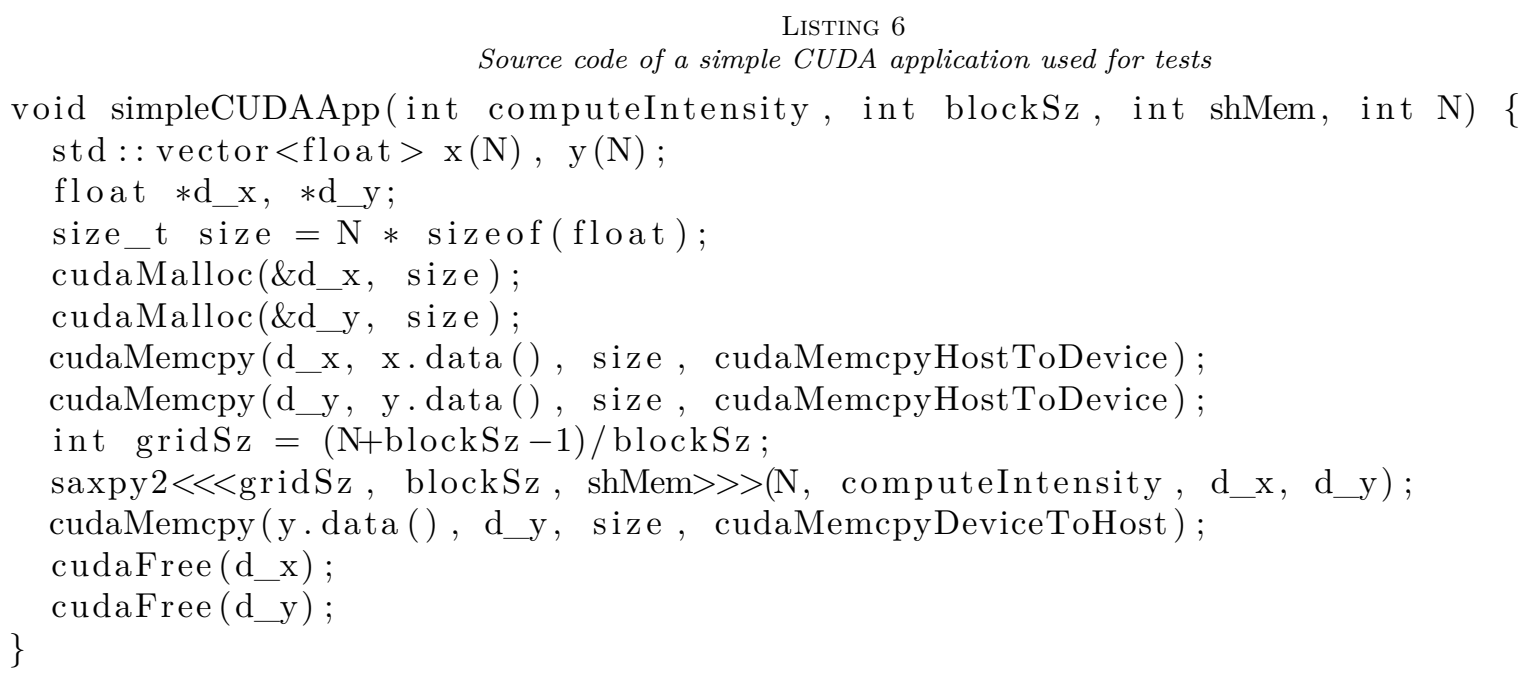

6.3. Tests and Results. In the first of the test scenarios we investigate the effect of a varying compute intensity of the kernel on its execution time. We have measured and simulated execution times for compute intensities ( $a$ parameter from Listing 2) spanning from 1 to 4096, while keeping all of the other parameters constant. This test verifies whether the theoretical model works for kernels exhibiting various ratios of computations to communication. For saxpy2 running with a maximum achievable occupancy of 64 warps per SM, if the intensity is low, then the kernel is bound by the throughput of a global memory, if it is high enough (32 in our case) it is bound by the CUDA cores throughput. We have observed that for all devices the model accurately determines the kernel execution time, however for lower compute intensities it is less precise, as the actual measured values diverge more from the predictions. This confirms that the model handles throughput bound scenario very well when the bound is imposed by the CUDA cores and is less accurate when it is due to the global memory system. The latter is not surprising as we have decided to take a simplified approach to the global memory modelling, by not considering the gradual saturation effect, using an equation to determine the theoretical memory throughput, and not adjusting it to the actual hardware characteristics.

The second test case verifies whether our implementation of the model handles two performance modes latency and throughput bound. To address both, we adjust the occupancy achieved when executing the kernel. To control the occupancy without modifying the block size we allocate dummy shared memory that effectively limits the maximum number of blocks that may be assigned to an SM. Note that it is not technically possible to cover every single value of the occupancy due to the fact how block and warp allocation works but nevertheless we were able to address a wide range of occupancies from 2 warps per SM to 64. Figure 6.1 shows the test results for Maxwell devices, the model precisely determines the kernel execution time with an average relative error of $5.4 \%$ and $7.8 \%$ for GTX 970 and GTX TITAN X respectively. For GTX 1070 the error was 3.9\%, we do not present it on this and subsequent figures for the sake of brevity. Furthermore, the transition from latency bound to throughput bound mode is represented accurately as well. Based on Fig. 6.1 we can determine the occupancy at which the transition occurs, this is around 32 warps / SM where rest of the figure becomes flat. However, for very small values of the occupancy the model overestimates the execution time by a small factor. Various block sizes were used when performing this test and it was noticed that the block size had no significant effect on the kernel execution time and the only significant factor was the occupancy. The validity of this conclusion is confirmed by the fact that what matters is the effectiveness of the utilisation of SM resources, which is directly related to achieved occupancy and not to the block size. The block size can only have an indirect effect by affecting the occupancy, which was not the case here once we excluded uncommon scenarios, where the size was smaller than 32. The proposed model is also based on calculation of the SM resources utilisation, therefore it behaved correctly in this scenario.

For the last test, we start by considering the kernel execution time. The charts showing the execution time in function of data size are depicted in Fig. 6.2 and Fig. 6.3, the former for small data sizes and the latter for large ones. Since the model was calibrated for a scenario with hundreds of thousands of elements it is less 


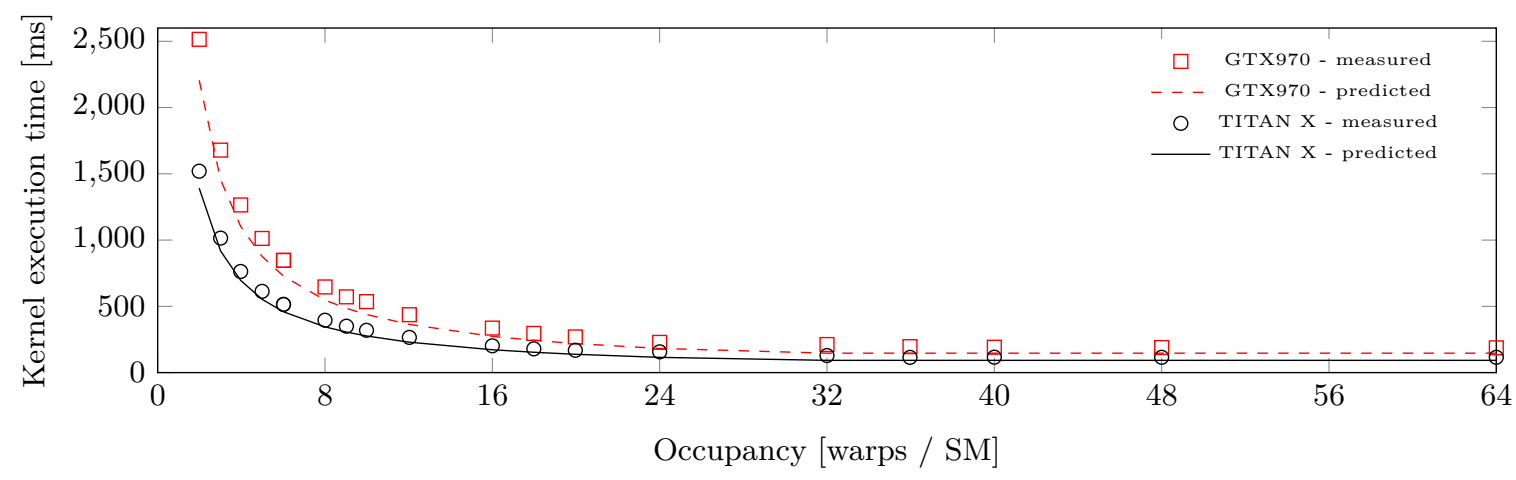

FIG. 6.1. Kernel execution time in function of occupancy

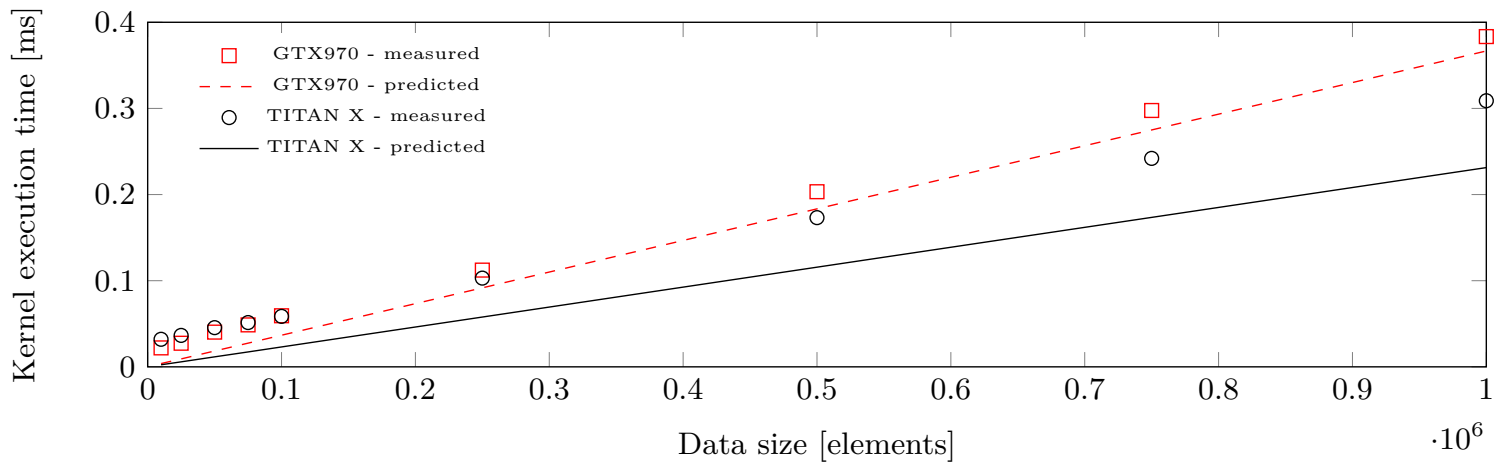

FiG. 6.2. Kernel execution time for small data sizes

accurate when the data size is small and improves its accuracy as the number of elements to process increases. The average error for large data size was 3.7\% (GTX 970), 4.6\% (GTX TITAN X) and 13.6\% (GTX 10\%0). The last one was expected to be higher since the model was calibrated for a different hardware architecture. The important fact is that even for the overestimated results, the functional relation between the data size and the execution time is maintained. This is acceptable since in real world scenarios the computations are performed for large data sizes and a case where the data is relatively small may be considered an uncommon one.

Since this test concerns a varying data size it must include data transfer time estimation, unlike the previous ones which focused only on the kernel execution. Given that our testbeds used different configurations of the PCIe bus we measured $\lambda_{c}$ and $t_{s}$ separately for each of them. The results are presented in Table 6.2, these were then used as the parameters of the hardware unit, which are substituted to Eq. 4.6 in the computational model.

Measured and estimated data transfer times are shown in Fig. 6.4. A decrease in accuracy for smaller data sizes resulting from an overestimation can be noticed, this is the same observation as in the case of the kernel execution time. Note that the transfers from host to the device take twice as much time because there are two arrays of $\mathrm{N}$ elements to be transferred in this direction, compared to only a single array that is transferred back. We have also proved experimentally here what was pointed out earlier, that the $\lambda_{c}$ and $t_{s}$ assume different values depending on the transfer direction. If this effect was not considered, then the accuracy of the results would be lower. For large data sizes the predicted values very closely resemble the measured ones, thus we may conclude that the methodology proposed for measuring data transfer times is valid.

Finally, the total execution time of the application for large data sizes is presented in Fig. 6.5. Technically, it sums up the results from the previous figures presented earlier in this section, since what comprises the application execution time is the data transfer in both directions and the kernel execution. It also concludes our work and proves its correctness by showing that the model we have implemented is capable of accurate 


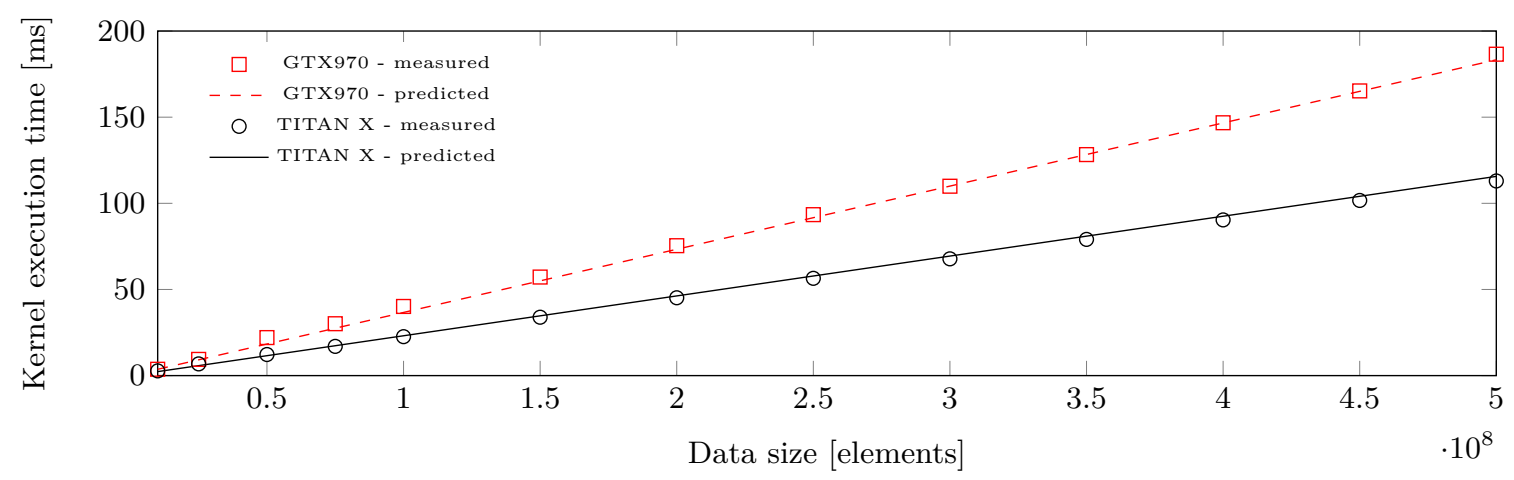

FIG. 6.3. Kernel execution time for large data sizes

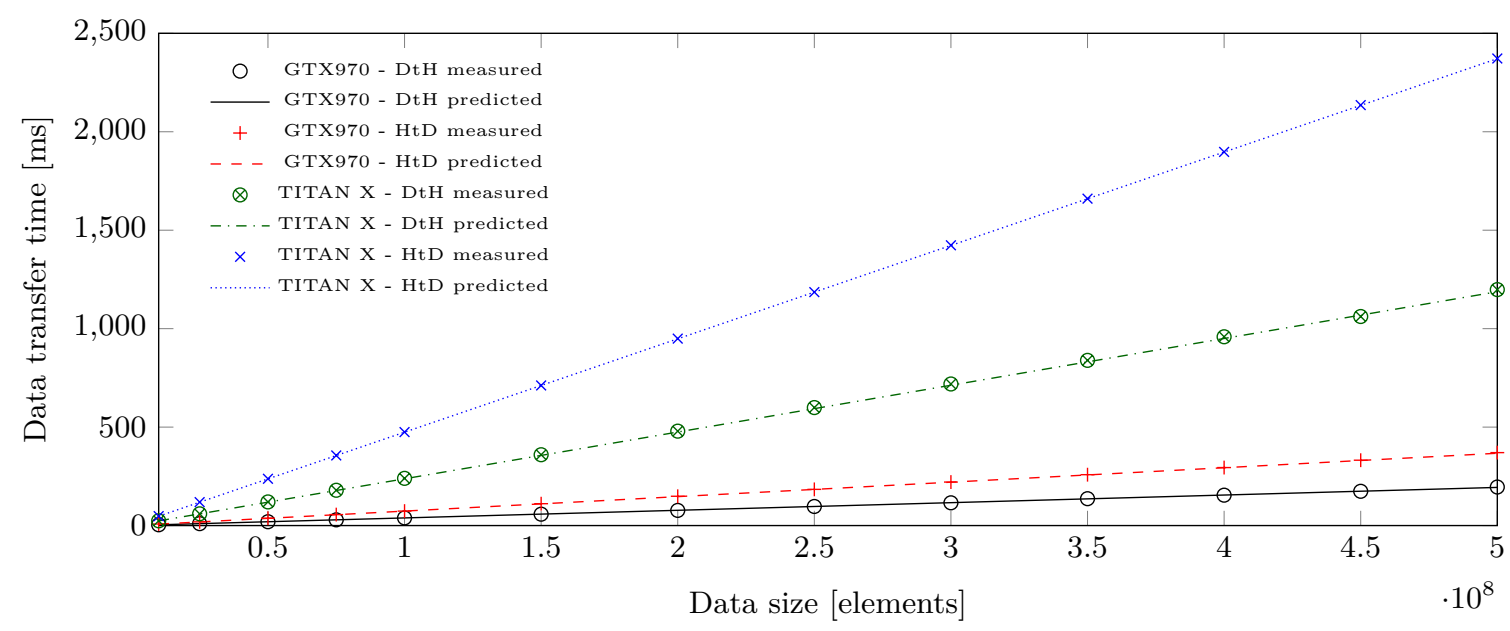

FIG. 6.4. Data transfer times for large data sizes

prediction of the application execution time for the most essential scenario, achieving $1.8 \%, 0.5 \%$ and $1.5 \%$ mean relative error for large data size $(\geq 10000000$ elements, where 1 element $=8$ Bytes as described in Section 5$)$, for tested GPUs respectively. From the end user perspective, parameters like occupancy, block size and compute intensity will likely be determined once and then kept constant. What is probably the most common real-world use case, is verification of the application on different hardware setups and for different data sizes.

6.4. Discussion. For the most essential scenario with a varying data size, when this size was not very small, the mean values of the error were $1.8 \%$ for GTX 970 and $0.5 \%$ for GTX TITAN X. Tests of a compute bound kernel with varying occupancy yielded an average prediction error of $5.4 \%$ and $7.8 \%$ respectively which is also a very good result. When the input data size was small or the kernel was bound by a memory throughput, the relative prediction error had a mean value of $32.8 \%$ for GTX 970 and $9.9 \%$ for GTX TITAN X. Significantly worse results for a memory throughput bound kernel are caused by a highly simplified memory access model. For compute throughput bound kernels, the error went down to a few percent depending on the launch configuration. It should be further noted that for every test case the functional relationship between the launch configuration parameter being investigated and the application execution time was very closely reproduced by the model.

7. Summary and Future Work. We have implemented adaptation of a performance model proposed by Volkov in the MERPSYS simulator for simulation of parallel applications on GPUs. We have verified the correctness of the model for various launch configurations and representative scenarios on three testbeds. MERPSYS has been proven to be an easily extendable and feasible tool for GPU modelling, and it was also 


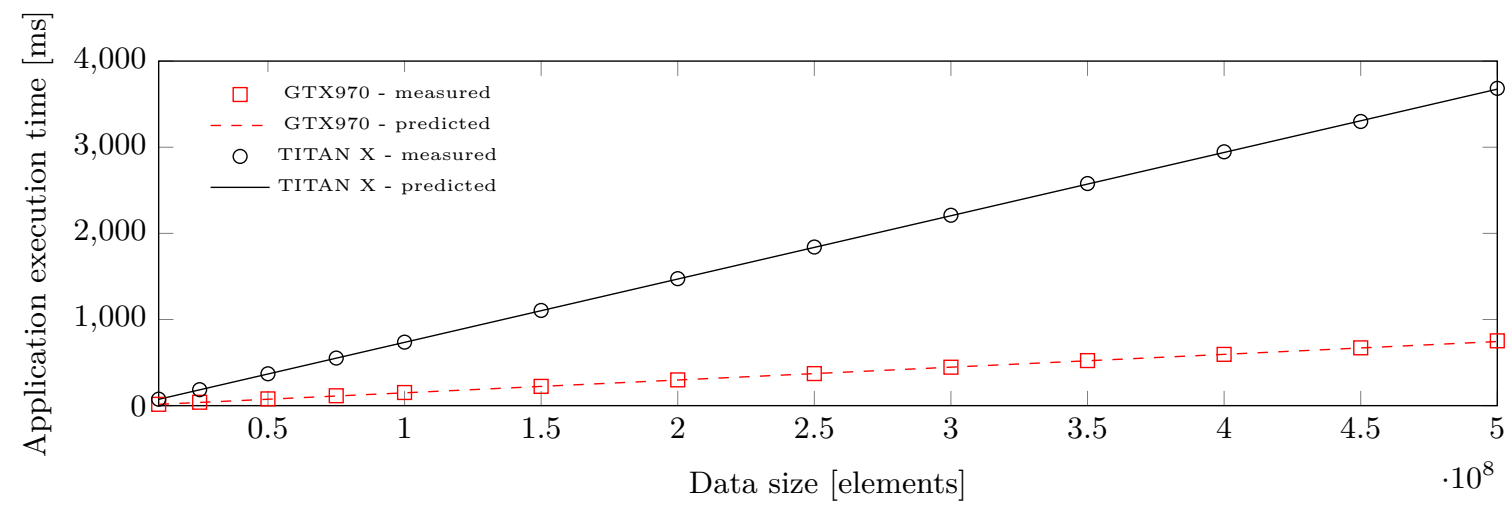

FIG. 6.5. Application execution times for large data sizes

shown that processing on a GPU may be conveniently modelled with a high degree of accuracy using a generalpurpose simulator. MERPSYS, with the deployed model, allows its users to assess the behaviour of their applications for data sizes exceeding the hardware capabilities of units available to them and even use ones that are not in their possession, given that these are available in MERPSYS' database. It allows to evaluate the application on hardware setups, on which testing prior to actual computational runs, would not otherwise be feasible, e.g. large clusters with a high cost per core-hour. It also allows to estimate the costs by predicting how long it will take for the computations to finish. In case of long-running applications, this allows for significantly shorter simulation times than the real runs.

The scope of modelling can be extended in the future as we have omitted double precision units, SFUs and shared memory, all of these could be fairly easy added to the model. Moreover, the approach to modelling of global memory accesses can be extended to consider caches, gradual saturation effect and varying access time depending on the transfer direction. So far we did include CUDA and NVIDIA GPUs in the model, possible extension includes its generalisation to be applicable to units produced by AMD and the OpenCL framework. The model can be extended with inclusion of an equation for occupancy calculation, which could be incorporated into the existing set of the equations and hence remove the need of relying on an external tool for this purpose. The solution would also largely benefit from an automation of the kernel analysis process. The behaviour of the model could also be verified on different GPU hardware architectures.

Acknowledgements. The work has been supported partially by the Polish Ministry of Science and Higher Education.

\section{REFERENCES}

[1] Top 500 list. [accessed November-2018].

[2] M. Amaris, D. Cordeiro, A. Goldman, and R. Y. D. Camargo, A simple bsp-based model to predict execution time in gpu applications, in 2015 IEEE 22nd International Conference on High Performance Computing (HiPC), Dec 2015, pp. 285-294.

[3] M. Amaris, R. Y. de Camargo, M. Dyab, A. Goldman, and D. Trystram, A comparison of gpu execution time prediction using machine learning and analytical modeling, in 2016 IEEE 15th International Symposium on Network Computing and Applications (NCA), Oct 2016, pp. 326-333.

[4] M. Andersch, J. Lucas, M. Alvarez-Mesa, And B. Juurlink, Analyzing gpgpu pipeline latency, in Proc. 10th Int. Summer School on Advanced Computer Architecture and Compilation for High-Performance and Embedded Systems, Fiuggi, Italy (ACACES' 14), July 2014

[5] S. S. Baghsorkhi, M. Delahaye, S. J. Patel, W. D. Gropp, and W.-M. W. Hwu, An adaptive performance modeling tool for gpu architectures, in Proceedings of the 15th ACM SIGPLAN Symposium on Principles and Practice of Parallel Programming, PPoPP '10, New York, NY, USA, 2010, ACM, pp. 105-114.

[6] A. Bakhoda, G. L. Yuan, W. W. L. Fung, H. Wong, and T. M. Aamodt, Analyzing cuda workloads using a detailed gpu simulator, in 2009 IEEE International Symposium on Performance Analysis of Systems and Software, April 2009, pp. 163-174. 
[7] S. Bąk, M. Krystek, K. Kurowski, A. Oleksiak, W. Piątek, and J. Węglarz, Gssim - a tool for distributed computing experiments, Sci. Program., 19 (2011), pp. 231-251.

[8] R. Buyya And M. Murshed, Gridsim: a toolkit for the modeling and simulation of distributed resource management and scheduling for grid computing, Concurrency and Computation: Practice and Experience, 14 (2002), pp. 1175-1220.

[9] R. N. Calheiros, R. Ranjan, A. Beloglazov, C. A. F. De Rose, and R. Buyya, Cloudsim: A toolkit for modeling and simulation of cloud computing environments and evaluation of resource provisioning algorithms, Softw. Pract. Exper., 41 (2011), pp. 23-50.

[10] N. CAPODIECI AND P. BURGIO, Efficient implementation of genetic algorithms on gp-gpu with scheduled persistent cuda threads, in 2015 Seventh International Symposium on Parallel Architectures, Algorithms and Programming (PAAP), Dec 2015, pp. 6-12.

[11] Z. Cui, Y. Liang, K. Rupnow, and D. Chen, An accurate gpu performance model for effective control flow divergence optimization, in 2012 IEEE 26th International Parallel and Distributed Processing Symposium, May 2012, pp. 83-94.

[12] P. Czarnul, ed., Modeling Large-Scale Computing Systems. Concepts and Models, Gdańsk University of Technology, Gdańsk, Poland, 2013.

[13] - ed., Modeling Large-Scale Computing Systems. Practical Approaches in MERPSYS, Gdańsk University of Technology, Gdańsk, Poland, 2016.

[14] P. Czarnul, J. Kuchta, and M. Matuszek, Parallel computations in the volunteer-based comcute system, in Parallel Processing and Applied Mathematics, R. Wyrzykowski, J. Dongarra, K. Karczewski, and J. Waśniewski, eds., Berlin, Heidelberg, 2014, Springer Berlin Heidelberg, pp. 261-271.

[15] P. Czarnul, J. Kuchta, M. Matuszek, J. Proficz, P. Rościszewski, M. Wójcik, and J. Szymański, Merpsys: An environment for simulation of parallel application execution on large scale hpc systems, Simulation Modelling Practice and Theory, 77 (2017), pp. $124-140$.

[16] P. Czarnul, J. Kuchta, P. Rościszewski, and J. Proficz, Modeling energy consumption of parallel applications, in 2016 Federated Conference on Computer Science and Information Systems (FedCSIS), Sept 2016, pp. 855-864.

[17] P. Czarnul, P. Rościszewski, M. Matuszek, and J. Szymański, Simulation of parallel similarity measure computations for large data sets, in 2015 IEEE 2nd International Conference on Cybernetics (CYBCONF), June 2015, pp. 472-477.

[18] P. Czarnul, K. Tomko, and H. Krawczyk, Dynamic partitioning of the divide-and-conquer scheme with migration in pvm environment, in Recent Advances in Parallel Virtual Machine and Message Passing Interface, Y. Cotronis and J. Dongarra, eds., Berlin, Heidelberg, 2001, Springer Berlin Heidelberg, pp. 174-182.

[19] T. T. Dao, J. Kim, S. Seo, B. Egger, And J. Lee, A performance model for gpus with caches, IEEE Transactions on Parallel and Distributed Systems, 26 (2015), pp. 1800-1813.

[20] W. E. Denzel, J. Li, P. Walker, And Y. Jin, A framework for end-to-end simulation of high-performance computing systems, in Proceedings of the 1st International Conference on Simulation Tools and Techniques for Communications, Networks and Systems \& Workshops, Simutools '08, ICST, Brussels, Belgium, Belgium, 2008, ICST (Institute for Computer Sciences, Social-Informatics and Telecommunications Engineering), pp. 21:1-21:10.

[21] B. Donassolo, H. Casanova, A. Legrand, and P. Velho, Fast and scalable simulation of volunteer computing systems using simgrid, in Proceedings of the 19th ACM International Symposium on High Performance Distributed Computing, HPDC '10, New York, NY, USA, 2010, ACM, pp. 605-612.

[22] M. HARRIS, An easy introduction to cuda c and c++, October 2012. [accessed November-2018].

[23] S. Hong AND H. KIM, An analytical model for a gpu architecture with memory-level and thread-level parallelism awareness, in Proceedings of the 36th Annual International Symposium on Computer Architecture, ISCA '09, New York, NY, USA, 2009, ACM, pp. 152-163.

[24] J. C. Huang, J. H. Lee, H. Kim, and H. H. S. Lee, Gpumech: Gpu performance modeling technique based on interval analysis, in 2014 47th Annual IEEE/ACM International Symposium on Microarchitecture, Dec 2014, pp. 268-279.

[25] W. Jia, K. A. Shaw, And M. Martonosi, Stargazer: Automated regression-based gpu design space exploration, in 2012 IEEE International Symposium on Performance Analysis of Systems Software, April 2012, pp. 2-13.

[26] Z. Jia, M. Maggioni, B. Staiger, and D. P. Scarpazza, Dissecting the NVIDIA Volta GPU Architecture via Microbenchmarking, ArXiv e-prints, (2018).

[27] A. KerR, G. Diamos, And S. Yalamanchili, A characterization and analysis of ptx kernels, in Workload Characterization, 2009. IISWC 2009. IEEE International Symposium on, oct. 2009, pp. 3 -12.

[28] - Modeling gpu-cpu workloads and systems, in Proceedings of the 3rd Workshop on General-Purpose Computation on Graphics Processing Units, GPGPU-3, New York, NY, USA, 2010, ACM, pp. 31-42.

[29] K. Kurowski, J. Nabrzyski, A. Oleksiak, and J. Węglarz, Gssim - grid scheduling simulator, Computational Methods in Science and Technology, 13 (2007), pp. 121-129.

[30] S. Madougou, A. Varbanescu, C. de LaAt, and R. van Nieuwpoort, The landscape of gpgpu performance modeling tools, Parallel Comput., 56 (2016), pp. 18-33.

[31] S. Madougou, A. L. Varbanescu, C. D. Laat, and R. V. Nieuwpoort, A tool for bottleneck analysis and performance prediction for gpu-accelerated applications, in 2016 IEEE International Parallel and Distributed Processing Symposium Workshops (IPDPSW), May 2016, pp. 641-652.

[32] O. Maitre, Understanding NVIDIA GPGPU Hardware, Springer-Verlag, Berlin, 2013.

[33] Micron Technology, Gddr5 sgram introduction, 2014.

[34] NVIDiA Corporation, Cuda occupancy calculator, December 2016. [accessed November-2018].

[35] — nvidia-smi - nvidia system management interface, July 2016. [accessed November-2018].

[36] — Cuda binary utilities, March 2017. [accessed November-2018].

[37] Cuda c programming guide, June 2017. [accessed November-2018]. 
[38] - Nvidia cuda compiler driver nvcc, March 2017. [accessed November-2018].

[39] _ Parallel thread execution isa, March 2017. [accessed November-2018].

[40] A. K. Parakh, M. Balakrishnan, and K. Paul, Performance estimation of gpus with cache, in 2012 IEEE 26th International Parallel and Distributed Processing Symposium Workshops PhD Forum, May 2012, pp. 2384-2393.

[41] S. F. Piraghaj, A. V. Dastjerdi, R. N. Calheiros, and R. Buyya, Containercloudsim: An environment for modeling and simulation of containers in cloud data centers, Software: Practice and Experience, 47 (2017), pp. 505-521. spe.2422.

[42] M. Quinson, Simgrid: a generic framework for large-scale distributed experiments, in 2009 IEEE Ninth International Conference on Peer-to-Peer Computing, Sept 2009, pp. 95-96.

[43] H. RASHIDI, Discrete simulation software: a survey on taxonomies, Journal of Simulation, 11 (2017), pp. 174-184.

[44] P. Rościszewski, Modeling and simulation for exploring power/time trade-off of parallel deep neural network training, Procedia Computer Science, 108 (2017), pp. 2463 - 2467. International Conference on Computational Science, ICCS 2017, 12-14 June 2017, Zurich, Switzerland.

[45] T. Scudiero, Memory bandwidth bootcamp: Best practices, in Proceedings of the GPU Technology Conference, GTC, 2015.

[46] - Memory bandwidth bootcamp: Beyond best practices, in Proceedings of the GPU Technology Conference, GTC, 2015.

[47] A. Siavashi And M. Momtazpour, Gpucloudsim: an extension of cloudsim for modeling and simulation of gpus in cloud data centers, The Journal of Supercomputing, (2018).

[48] A. Sulistio, C. S. Yeo, And R. BuYya, A taxonomy of computer-based simulations and its mapping to parallel and distributed systems simulation tools, Softw., Pract. Exper., 34 (2004), pp. 653-673.

[49] L. G. Valiant, A bridging model for parallel computation, Commun. ACM, 33 (1990), pp. 103-111.

[50] V. Volkov, Better performance at lower occupancy, in Proceedings of the GPU Technology Conference, GTC, vol. 10, 2015.

[51] — Understanding Latency Hiding on GPUs, PhD thesis, EECS Department, University of California, Berkeley, Aug 2016.

[52] G. Wu, J. L. Greathouse, A. Lyashevsky, N. Jayasena, and D. Chiou, Gpgpu performance and power estimation using machine learning, in 2015 IEEE 21st International Symposium on High Performance Computer Architecture (HPCA), Feb 2015, pp. 564-576.

[53] Y. ZhANG AND J. D. Owens, A quantitative performance analysis model for gpu architectures, in 2011 IEEE 17th International Symposium on High Performance Computer Architecture, Feb 2011, pp. 382-393.

Edited by: Dana Petcu

Received: Sep 7, 2018

Accepted: Nov 3, 2018 


\title{
A COMPARISON OF MESSAGE PASSING INTERFACE (MPI) AND CO-ARRAY FORTRAN FOR LARGE FINITE ELEMENT VARIABLY SATURATED FLOW SIMULATIONS *
}

\author{
FRED T. TRACY† THOMAS C. OPPE $\ddagger$ AND MAUREEN K. CORCORAN§
}

\begin{abstract}
The purpose of this research is to determine how well co-array FORTRAN (CAF) performs relative to Message Passing Interface (MPI) on unstructured mesh finite element groundwater modelling applications with large problem sizes and core counts. This research used almost 150 million nodes and 300 million 3-D prism elements. Results for both the Cray XE6 and Cray XC30 are given. A comparison of the ghost-node update algorithms with source code provided for both MPI and CAF is also presented.
\end{abstract}

Key words: Co-array FORTRAN, MPI, finite element method, variably saturated seepage flow modelling

AMS subject classifications. 35J66,65Y05, 76S05

1. Introduction. Several parallel programming paradigms have been developed in recent years as alternatives to the popular software Message Passing Interface (MPI) [1] used for passing messages among the processes of a distributed memory parallelized program. One of these new ways is the Partitioned Global Address Space (PGAS) [2] paradigm where arrays partitioned across processes can be referenced by special syntax implemented in the language. A popular PGAS language for FORTRAN users is co-array FORTRAN (CAF) [3], and a CAF specification has been adopted in the FORTRAN 2008 standard. CAF has performed better than MPI for certain applications, and it was found easier to program than MPI. A recent example tested structured-grid partial-differential-equation applications [4].

A recent paper [7] describing the challenges and scalability results of running a large finite element model of variably saturated flow [5] in a three-dimensional (3-D) levee on a large high performance, parallel computer where MPI was used for the communication was published. Using the same levee model, this current research expands that work by using CAF for the communication and comparing these results with the results using MPI. The original finite element model consisted of 3,017,367 nodes and 5,836,072 3-D prism elements running on 32 cores, and the problem and core count were magnified as much as 350 times to achieve 1,044,246,303 nodes and 2,042,625,200 elements.

A traditional partitioning of the mesh achieved approximately the same number of finite element nodes on each core. Thus, the main communication challenge was updating ghost-node information on the different cores for a solution of a system of simultaneous, linear equations at each nonlinear iteration. In both the MPI and CAF versions, the ghost node data are first buffered and then sent to the different cores where they are needed. Details of the FORTRAN coding for both MPI and CAF are described herein.

2. Description of the problem. The problem consists of steady-state seepage flow through a levee as shown in Fig. 2.1 and idealised in Fig. 2.2 where there are several soil layers. A detailed description of this problem is given in $[6,7]$. The challenges of parallelization using MPI of the groundwater program used in this research, when the problem size is approximately one billion nodes and two billion elements, are given in [7]. Performance results are also given. Fig. 2.3 shows a portion of the 3 -D mesh of the levee system before a tree with its root system was added at the toe. More details of the modelling of the woody vegetation are given in [6]. To model the tree root at the toe of the levee, a $5 \mathrm{ft} \times 6 \mathrm{ft} \times 6 \mathrm{ft}$ heterogeneous zone was added in which the mesh was refined using 1 in $\times 1$ in $\times 1$ in 3 -D prism elements (Fig. 2.4). To simulate heterogeneities, a randomly generated hydraulic conductivity was assigned to each element in this zone. The resulting mesh consisted of 3,017,367 nodes and 5,836,072 3-D prism elements.

\footnotetext{
${ }^{*}$ This work was supported in part by a grant of computer time from the Department of Defense High Performance Computing Modernization Program (HPCMP).

${ }^{\dagger}$ Information Technology Laboratory (ITL), Engineer Research and Development Center (ERDC), Vicksburg, MS.

${ }^{\ddagger}$ ITL, ERDC, Vicksburg, MS.

$\S$ Geotechnical and Structures Laboratory, ERDC, Vicksburg, MS.
} 


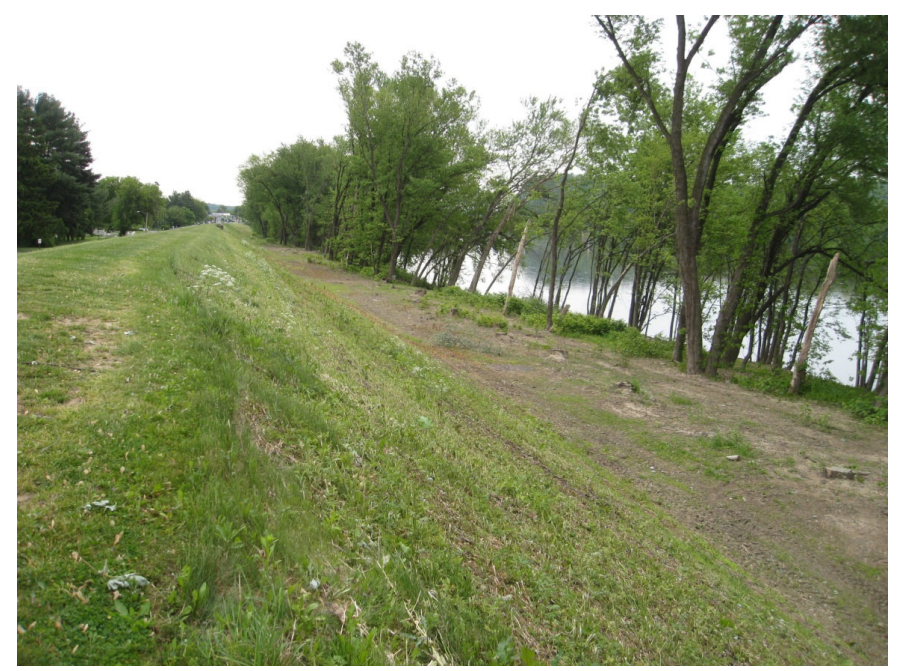

FIG. 2.1. River side of a levee with trees.

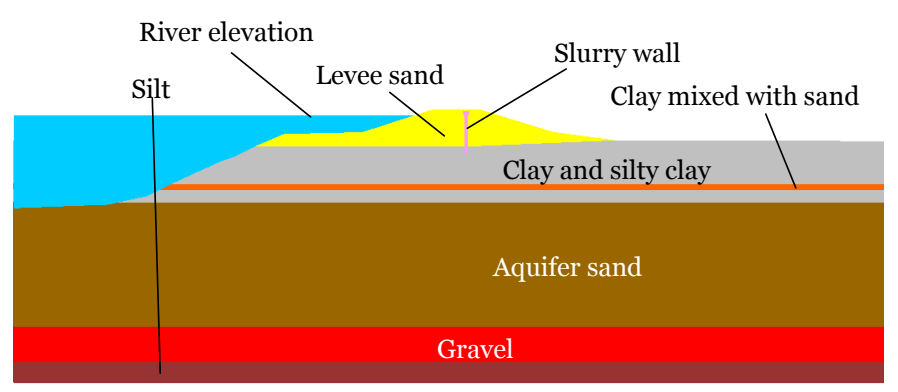

FIG. 2.2. Cross section of a levee with material types and elevation of the river.

3. High performance parallel computing. The parallel 3-D groundwater finite element program mentioned above was run on Garnet, the Cray XE6 at the U.S. Army Engineer Research and Development Center (ERDC) [8], and on Lightning, the Cray XC30 at the Air Force Research Laboratory, Aberdeen, MD [9]. At the time of this study, Garnet consisted of 4,716 dual-socket compute nodes with each socket populated with a 2.5 GHz 16-core AMD 6200 Opteron (Interlagos) processor. Each node had 64 GB memory (60 GB user-accessible) or an average of 1.875 GB memory per core. The interconnect type was Cray Gemini in a 3-D torus topology. Garnet was rated at 1.5 peak PFLOPS or 10 GFLOPS per core when these computations were done. Garnet had a large Lustre file system that was tuned for parallel I/O. At the time of this research, Lightning consisted of 2,360 dual-socket compute nodes with each socket populated with a $2.7 \mathrm{GHz} 12$-core Intel Xeon E5-2697v2 (Ivy Bridge) processor. Each node had 64 GB memory (63 GB user-accessible) or an average of 2.625 GB memory per core. The interconnect type was Cray Aries in a Dragonfly topology. Lightning was rated at 1.2 peak PFLOPS or 21.6 GFLOPS per core when the data in this paper were collected. Lightning had a large Lustre file system that was also tuned for parallel I/O.

The parallelization of the 3-D seepage/groundwater program was separated into four parts or programs. One MPI process or one CAF image was placed on each core of a compute node. The four programs are (1) a partitioner using the Parallel Graph Partitioning and Fill-reducing Matrix Ordering program, ParMETIS [10], to divide the mesh into approximately equal pieces among the MPI processes or CAF images, (2) a preparer to provide data, such as owned nodes, ghost nodes, owned elements, ghost elements, and communication data, needed for each MPI process or CAF image, (3) a finite element program that does the finite element computations with output files containing results for each owned node of that MPI process or CAF image, and (4) a post processor that combines all data from each MPI process or CAF image into the final output files. 


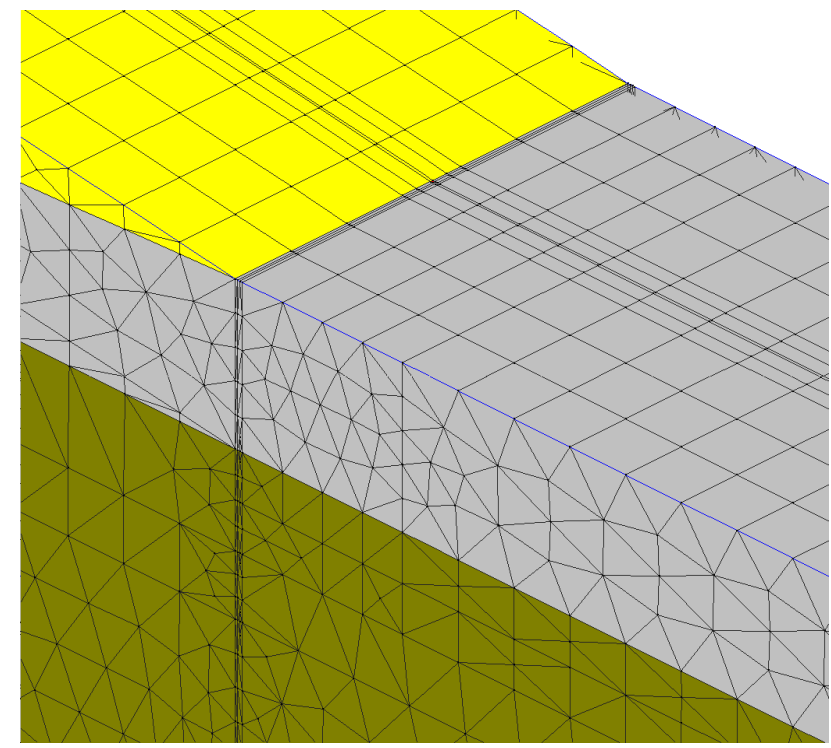

FIG. 2.3. Portion of the 3-D mesh before the root zone was added.

The primary communication challenge is ghost node updates in the conjugate gradient or BICG-STAB [11] linear solvers using either a Picard or Newton linearization $[12,13]$ of the governing nonlinear equation of Richards' equation [14]. As in [7], only times to solution for the finite element part of the program suite were collected for MPI and CAF and reported in this paper. The ghost node update routine for both MPI and CAF is examined in detail in the following section.

\section{Ghost node update.}

4.1. MPI. Table 4.1 gives the ghost node update subroutine for MPI and a description of the important variables. The subroutine has three steps: (1) a set-up phase in which data are to be received from the different cores, (2) send data to the different cores, and (3) wait until all the MPI messages have been processed. The arrays, nstngh, ighost, and nodgh have all been supplied by the preparer program. There are no global arrays in the parallel versions of the finite element program, i.e., no arrays with sizes of the total number of nodes or the total number of elements. The elimination of global arrays allowed for much larger finite element meshes to be run.

4.2. CAF. Table 4.2 gives the ghost node update subroutine for CAF and a description of the important variables. The same data provided in the MPI version were also provided to the CAF subroutine. The CAF version of ghost node updating is simpler than the MPI version in that for CAF, data are first placed in a buffer and then directly "put" into the different cores by the statement,

vc(nst : nst + num -1$)[i]=\operatorname{buff}(:)$

While in the MPI case, the efficiency of the data transfer is dependent on how well MPI_IRECV, MPI_SEND, and MPI_WAIT are implemented, the efficiency of the CAF routine is dependent on the quality of the FORTRAN compiler implementation and internal data transfer capability. It is also important to note that two explicit barrier calls,

call sync_all

were required in the CAF implementation, whereas none were required in the MPI version. Also, the huge page option described by

module load craype-hugepages2M

was required to run the CAF version. 


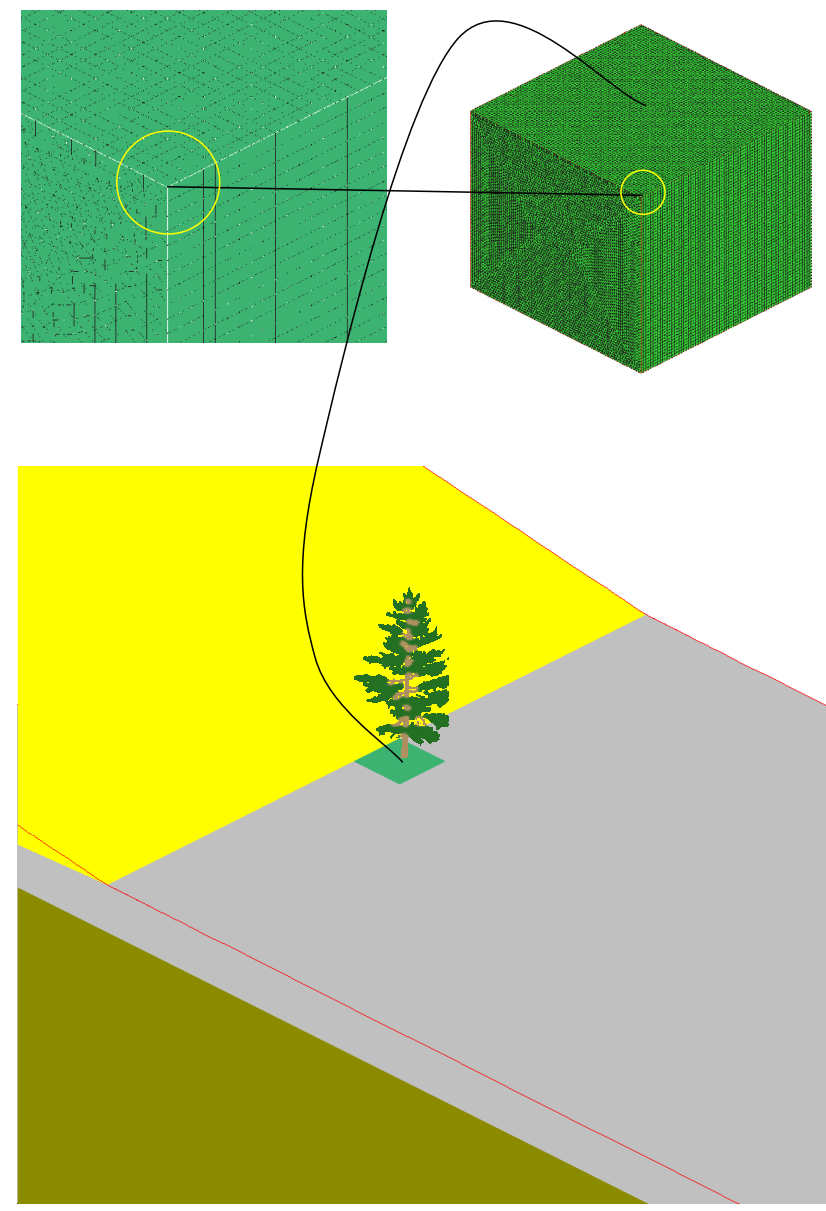

FIG. 2.4. Heterogeneous zone representing the roots of a tree.

5. Results and Analysis. All runs on both computers were done using the Cray compiler with -O1 optimisation.

5.1. Results. Tables 5.1 and 5.2 give the time to solution for the finite element program on the Cray XE6 and XC30 for different problem sizes and core counts. The $m$ represents how much the original problem is magnified to produce larger problem sizes. When $m=2$, for instance, two original meshes are created and joined such that the number of elements is exactly doubled, and the number of nodes is doubled less one set of the nodes common to the two pieces. The original problem was run with 96 cores. Although the MPI version of the finite element program could run this problem on 32 cores, the first multiple of 32 where the CAF version would run was 96 . Values of $m=1,2,5,10,20$, and 50 were run. The running times for MPI and CAF for three runs, their respective averages, and the ratio of MPI to CAF running times were tabulated for each $m$ value and core count.

5.1.1. Analysis. The following observations are made:

- When $m=1$ and the number of cores is 96, the ratio of MPI / CAF running times was almost equal.

- As $m$ increased, this ratio got significantly smaller. The ratios become so small that $m$ was not increased further than 50. Apparently, the global synchronisations required by the CAF implementation became increasingly costly as the partition size grew.

- The MPI/CAF ratio is larger for the XE6 than the XC30.

- The XC30 running times are approximately half of those of the XE6. 


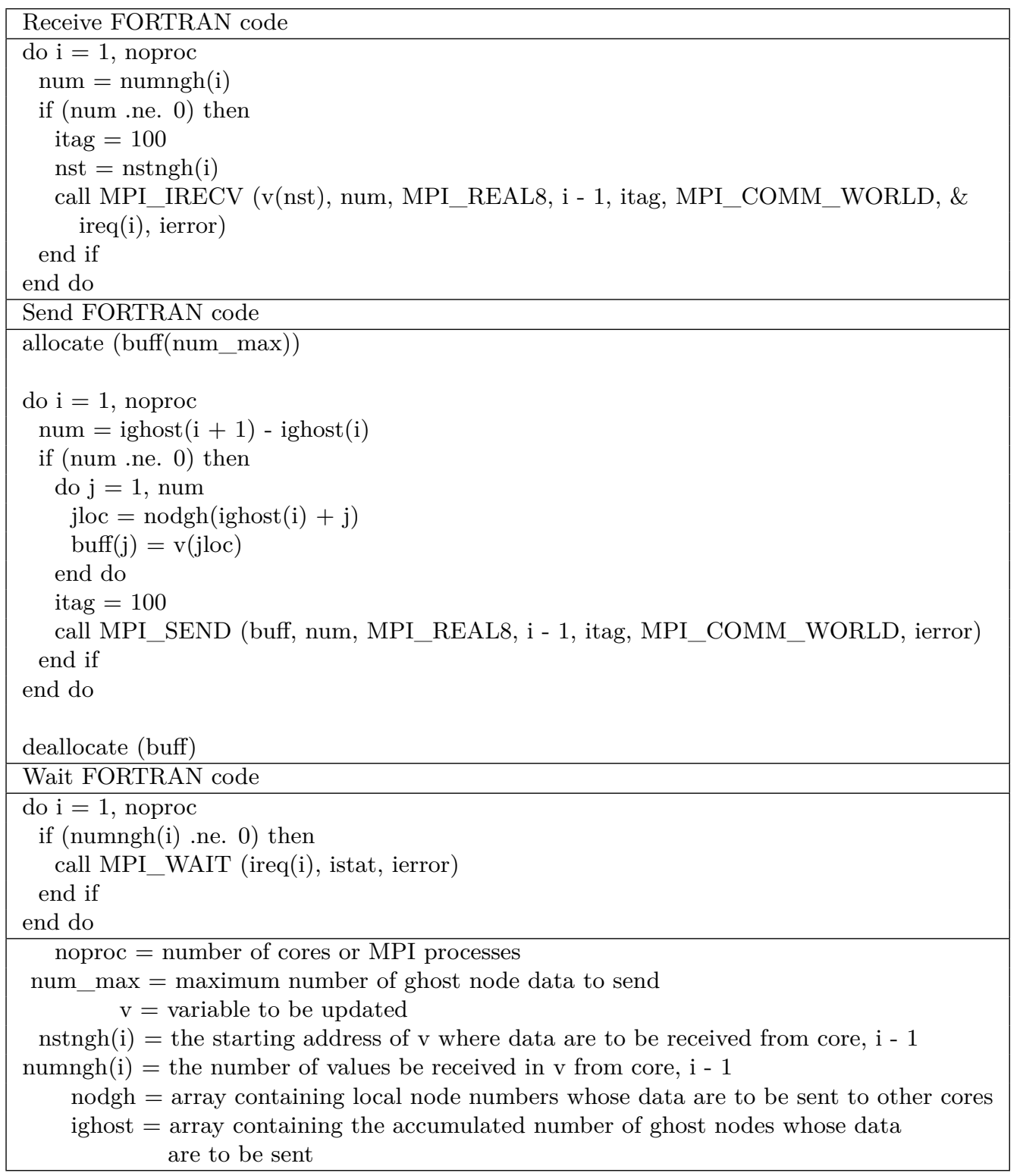


TABLE 4.2

Ghost node update for $C A F$.

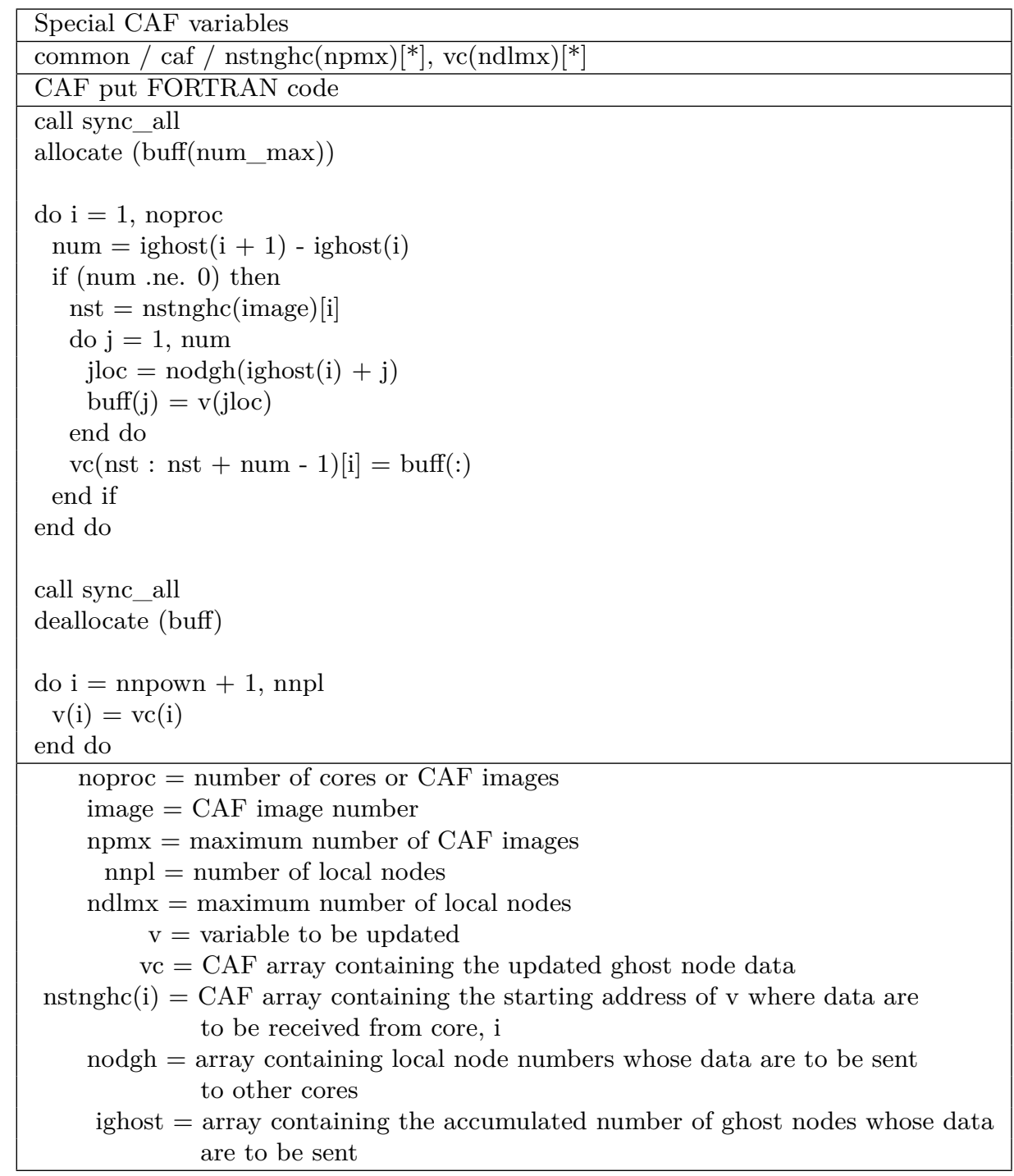


TABLe 5.1

Time (sec) for MPI and CAF on the Cray XE6 and XC30 for $m=1,2$, and 5 .

\begin{tabular}{|c|c|c|c|c|c|c|c|c|c|}
\hline$m$ & Nodes & Elements & Cores & Cray & \multicolumn{2}{|c|}{$\begin{array}{l}\text { Time } \\
\text { MPI }\end{array}$} & \multicolumn{2}{|c|}{$\begin{array}{l}\text { Time } \\
\text { CAF }\end{array}$} & $\begin{array}{c}\text { Ratio } \\
\text { MPI/CAF }\end{array}$ \\
\hline 1 & \multirow{2}{*}{3017367} & \multirow{2}{*}{5836072} & \multirow{2}{*}{96} & XE6 & \multirow{2}{*}{\multicolumn{2}{|c|}{$\begin{array}{l}788.0 \\
323.6\end{array}$}} & \multicolumn{2}{|c|}{816.0} & \\
\hline 1 & & & & XC30 & & & & 5.6 & \\
\hline & & & & XE6 & \multicolumn{2}{|c|}{786.6} & \multicolumn{2}{|c|}{826.7} & \\
\hline & & & & XC30 & \multicolumn{2}{|c|}{324.8} & \multicolumn{2}{|c|}{379.2} & \\
\hline & & & & XE6 & \multirow{2}{*}{\multicolumn{2}{|c|}{$\begin{array}{l}767.7 \\
322.1\end{array}$}} & \multicolumn{2}{|c|}{820.7} & \\
\hline & & & & XC30 & & & \multicolumn{2}{|c|}{375.3} & \\
\hline & & & & XE6 & \multirow{2}{*}{ Avg. } & 780.8 & \multirow{2}{*}{ Avg. } & 820.8 & 0.95 \\
\hline & & & & XC30 & & 323.5 & & 377.0 & 0.86 \\
\hline & & & & XE6 & \multirow{2}{*}{\multicolumn{2}{|c|}{$\begin{array}{l}601.1 \\
252\end{array}$}} & \multicolumn{2}{|c|}{658.8} & \\
\hline & & & 128 & XC30 & & & & 2.6 & \\
\hline & & & & XE6 & & & & 3.7 & \\
\hline & & & & XC30 & & & & 5.4 & \\
\hline & & & & XE6 & & & & 8.9 & \\
\hline & & & & XC30 & & & & 3.3 & \\
\hline & & & & XE6 & $A_{\mathrm{Y}} \boldsymbol{\gamma}$ & 598.3 & $A_{y} \sigma$ & 670.5 & 0.89 \\
\hline & & & & XC30 & Avg. & 255.8 & Avg. & 330.4 & 0.77 \\
\hline 8 & $600 \cap 821$ & 11672144 & 102 & XE6 & & & & 8.7 & \\
\hline 2 & 6000831 & 11672144 & 192 & XC30 & & & & 3.6 & \\
\hline & & & & XE6 & & & & 0.2 & \\
\hline & & & & XC30 & & & & 3.7 & \\
\hline & & & & XE6 & & & & 5.4 & \\
\hline & & & & XC30 & & & & 7.8 & \\
\hline & & & & XE6 & $A_{y x}$ & 793.1 & $A_{y x}$ & 925.1 & 0.86 \\
\hline & & & & XC30 & Avg. & 282.0 & Avg. & 385.0 & 0.73 \\
\hline & & & 256 & XE6 & & & & 4.4 & \\
\hline & & & 256 & XC30 & & & & 8.5 & \\
\hline & & & & XE6 & & & & 2.1 & \\
\hline & & & & XC30 & & & & 3.5 & \\
\hline & & & & XE6 & & & & 7.5 & \\
\hline & & & & XC30 & & & & $\begin{array}{l}6.7 \\
\end{array}$ & \\
\hline & & & & XE6 & $A=0$ & 645.2 & $A_{-1}$ & 841.3 & 0.76 \\
\hline & & & & XC30 & Avg. & 256.6 & Avg. & 392.9 & 0.65 \\
\hline 5 & 1405120 & 20180260 & 180 & XE6 & & & & 1.8 & \\
\hline 5 & 14951223 & 29180300 & 480 & XC30 & & & & 5.8 & \\
\hline & & & & XE6 & & & & 2.5 & \\
\hline & & & & XC30 & & & & 0.1 & \\
\hline & & & & XE6 & & & & 7.3 & \\
\hline & & & & XC30 & & & & 5.3 & \\
\hline & & & & XE6 & $A_{Y x}$ & 842.2 & $A_{1}$ & 1313.9 & 0.64 \\
\hline & & & & XC30 & Avg. & 346.1 & Avg. & 634.1 & 0.55 \\
\hline & & & 640 & XE6 & & & & 1.8 & \\
\hline & & & 040 & XC30 & & & & 5.3 & \\
\hline & & & & XE6 & & & & 4.0 & \\
\hline & & & & XC30 & & & & 1.2 & \\
\hline & & & & XE6 & & & & 1.4 & \\
\hline & & & & XC30 & & & & 5.9 & \\
\hline & & & & XE6 & A & 657.3 & $A_{Y x}$ & 1328.1 & 0.49 \\
\hline & & & & XC30 & Avg. & 264.1 & Avg. & 614.5 & 0.43 \\
\hline
\end{tabular}


TABLE 5.2

Time (sec) for MPI and CAF on the Cray XE6 and XC30 for $m=10,20$, and 50.

\begin{tabular}{|c|c|c|c|c|c|c|c|c|c|}
\hline$m$ & Nodes & Elements & Cores & Cray & \multicolumn{2}{|c|}{$\begin{array}{l}\text { Time } \\
\text { MPI }\end{array}$} & \multicolumn{2}{|c|}{$\begin{array}{l}\text { Time } \\
\text { CAF }\end{array}$} & $\begin{array}{c}\text { Ratio } \\
\text { MPI/CAF }\end{array}$ \\
\hline \multirow{8}{*}{10} & \multirow{2}{*}{3017367} & \multirow{2}{*}{58360720} & \multirow{2}{*}{960} & XE6 & \multirow{2}{*}{\multicolumn{2}{|c|}{$\begin{array}{l}862.0 \\
344.7\end{array}$}} & \multicolumn{2}{|c|}{$\begin{array}{c}1691.6 \\
9107\end{array}$} & \\
\hline & & & & XC30 & & & & 0.7 & \\
\hline & & & & XE6 & \multicolumn{2}{|c|}{881.2} & \multicolumn{2}{|c|}{1730.2} & \\
\hline & & & & XC30 & \multicolumn{2}{|c|}{353.1} & \multicolumn{2}{|c|}{912.2} & \\
\hline & & & & XE6 & \multirow{2}{*}{\multicolumn{2}{|c|}{$\begin{array}{l}867.5 \\
349.5\end{array}$}} & \multicolumn{2}{|c|}{1473.4} & \\
\hline & & & & XC30 & & & \multicolumn{2}{|c|}{907.5} & \\
\hline & & & & XE6 & \multirow{2}{*}{ Avg. } & 870.2 & \multirow{2}{*}{ Avg. } & 1631.7 & 0.53 \\
\hline & & & & XC30 & & 349.1 & & 910.1 & 0.38 \\
\hline & & & 1200 & XE6 & \multirow{2}{*}{\multicolumn{2}{|c|}{$\begin{array}{l}632.9 \\
266.9\end{array}$}} & \multicolumn{2}{|c|}{1309.0} & \\
\hline & & & 1280 & XC30 & & & & 5.1 & \\
\hline & & & & XE6 & & & & 34.0 & \\
\hline & & & & XC30 & & & & 9.3 & \\
\hline & & & & XE6 & & & & 88.6 & \\
\hline & & & & XC30 & & & & 8.2 & \\
\hline & & & & XE6 & & 622.3 & & 1553.9 & 0.40 \\
\hline & & & & XC30 & Avg. & 267.4 & Avg. & 997.5 & 0.27 \\
\hline 80 & 50702182 & 116701440 & 1020 & XE6 & & & & 35.8 & \\
\hline 20 & 59703183 & 116721440 & 1920 & XC30 & & & & 30.3 & \\
\hline & & & & XE6 & & & & 31.6 & \\
\hline & & & & XC30 & & & & 55.0 & \\
\hline & & & & XE6 & & & & 0.2 & \\
\hline & & & & XC30 & & & & 26.6 & \\
\hline & & & & XE6 & Avo & 894.9 & $A_{\mathrm{Yo}}$ & 2825.9 & 0.32 \\
\hline & & & & XC30 & Avg. & 357.1 & Avg. & 1374.0 & 0.26 \\
\hline & & & 2560 & XE6 & & & & 9.2 & \\
\hline & & & 2560 & XC30 & & & & 6.1 & \\
\hline & & & & XE6 & & & & 11.7 & \\
\hline & & & & XC30 & & & & 65.5 & \\
\hline & & & & XE6 & & & & 33.0 & \\
\hline & & & & XC30 & & & & 3.7 & \\
\hline & & & & XE6 & Ave & 654.3 & Ave & 2511.3 & 0.26 \\
\hline & & & & XC30 & Avg. & 275.8 & Avg. & 1575.1 & 0.18 \\
\hline 50 & 140207102 & 201802600 & 4800 & XE6 & & & & 22.1 & \\
\hline 50 & 149207103 & 291803600 & 4800 & XC30 & & & & 80.1 & \\
\hline & & & & XE6 & & & & 84.6 & \\
\hline & & & & XC30 & & & & 33.1 & \\
\hline & & & & XE6 & & & & 55.3 & \\
\hline & & & & XC30 & & & & 10.2 & \\
\hline & & & & XE6 & Ave & 896.3 & A & 5400.7 & 0.17 \\
\hline & & & & XC30 & Avg. & 363.6 & Avg. & 3087.8 & 0.12 \\
\hline & & & 6400 & XE6 & & & & 2.8 & \\
\hline & & & 6400 & $\mathrm{XC30}$ & & & & 1.3 & \\
\hline & & & & XE6 & & & & 2.2 & \\
\hline & & & & XC30 & & & & 33.0 & \\
\hline & & & & XE6 & & & & 66.4 & \\
\hline & & & & XC30 & & & & 66.7 & \\
\hline & & & & XE6 & A ver & 730.6 & A & 6317.1 & 0.12 \\
\hline & & & & XC30 & Avg. & 303.2 & Avg. & 3810.3 & 0.08 \\
\hline
\end{tabular}


TABLE 6.1

Consistency check comparing values of pressure head from the original mesh and the mesh for $m=50$ for the first 10 nodes and last 6 nodes of each mesh.

\begin{tabular}{|c|c|c|c|c|c|}
\hline \multicolumn{3}{|c|}{$m=1$} & \multicolumn{3}{c|}{$m=50$} \\
\hline Node & MPI & CAF & Node & MPI & CAF \\
\hline 1 & 129.00000 & 129.00000 & 1 & 129.00000 & 129.00000 \\
\hline 2 & 128.99678 & 128.99678 & 2 & 128.99678 & 128.99678 \\
\hline 3 & 128.99345 & 128.99345 & 3 & 128.99345 & 128.99345 \\
\hline 4 & 119.00000 & 119.00000 & 4 & 119.00000 & 119.00000 \\
\hline 5 & 118.99735 & 118.99735 & 5 & 118.99735 & 118.99735 \\
\hline 6 & 124.23808 & 124.23808 & 6 & 124.23808 & 124.23808 \\
\hline 7 & 118.99464 & 118.99464 & 7 & 118.99464 & 118.99464 \\
\hline 8 & 123.54520 & 123.54520 & 8 & 123.54520 & 123.54520 \\
\hline 9 & 128.98993 & 128.98993 & 9 & 128.98993 & 128.98993 \\
\hline 10 & 110.50000 & 110.50000 & 10 & 110.50000 & 110.50000 \\
\hline 6000826 & 0.0000000 & 0.0000000 & 149207098 & 0.0000000 & 0.0000000 \\
\hline 6000827 & 0.041718483 & 0.041718484 & 149207099 & 0.041718479 & 0.041718479 \\
\hline 6000828 & 3.0365778 & 3.0365778 & 149207100 & 3.0365779 & 3.0365779 \\
\hline 6000829 & 0.036494873 & 0.036494870 & 149207101 & 0.036494875 & 0.036494875 \\
\hline 6000830 & 0.0000000 & 0.0000000 & 149207102 & 0.0000000 & 0.0000000 \\
\hline 6000831 & 0.0000000 & 0.0000000 & 149207103 & 0.0000000 & 0.0000000 \\
\hline
\end{tabular}

6. Consistency check. A check for consistency for the first 10 nodes and last 6 nodes of the meshes for $m=1$ and $m=50$ was done with pressure head results placed in Table 6.1. The MPI and CAF results should be the same and because of symmetry, the values for $m=1$ and $m=50$ should also be the same. A comparison of the MPI and CAF results in Table 6.1 shows excellent consistency.

7. Conclusions. The following conclusions can be drawn:

1. Both MPI and CAF versions ran successfully and gave the same results.

2. As the problem size and process count increased, the results remained consistent.

3. The update routine for CAF was simpler than that for MPI.

4. CAF required more memory than MPI to run the same size mesh.

5. CAF required huge pages, but MPI did not.

6. CAF required explicit barriers, but MPI did not.

7. For the original problem, CAF and MPI performed almost the same when using 96 cores.

8. As the problem size and process count grew, MPI performed much better than CAF.

9. The MPI/CAF ratio is larger for the XE6 than the XC30.

10. The XC30 running times are approximately half of those of the XE6.

\section{REFERENCES}

[1] Message Passing Interface Forum, MPI: A Message-Passing Interface Standard, Version 3.0, http://www.mpiforum.org/docs/mpi-3.0/mpi30-report.pdf, 2012.

[2] PGAS, Partitioned Global Address Space, http://pgas.org, 2015.

[3] R. W. Numrich AND J. K. ReID, Co-arrays in the next FORTRAN Standard, Scientific Programming, 14 (2006), pp. 1-18.

[4] S. Garain, D. S. Balsara, AND J. Reid, Comparing Co-array FORTRAN (CAF) with MPI for several structured mesh PDE applications, J. of Comp. Physics, 297 (2015), pp. 237-253.

[5] J. IsTOK, Groundwater modelling by the finite element method, AGU, 1989.

[6] M. Corcoran, J. Peters, J. Dunbar, J. Llopis, F. Tracy, J. Wibowo, J. Simms, C. Kees, S. McKay, J. Fischenich, M. Farthing, M. Glynn, B. Robbins, R. Strange, M. Schultz, J. Clarke, T. Berry, C. Little, and L. Lee, Initial research into the effects of woody vegetation on levees, volume I of IV: project overview, volume II of IV: field data collection, volume III of IV: numerical model simulation, and volume IV of IV: summary of results and conclusions, U.S. Army Engineer Research and Development Center, Vicksburg, MS, 2011.

[7] F. T. Tracy, T. C. OpPe, W. A. WARD, AND M. K. Corcoran, A scalability study using supercomputers for huge finite element variably saturated flow simulations, Scalable Computing: Practice and Experience, 16 (2015), pp. 153-162. 
[8] ERDC DSRC, http://www.erdc.hpc.mil/hardware/index.html, Department of Defense Supercomputing Resource Center, Vicksburg, MS, 2014

[9] AFRL DSRC, http://www.afrl.hpc.mil/index.html, Department of Defense Supercomputing Resource Center, Aberdeen, MD, 2014.

[10] G. KarYPIS, ParMETIS - Parallel Graph Partitioning and Fill-reducing Matrix Ordering, http://glaros.dtc.umn.edu/ gkhome/metis/parmetis/overview, 2014.

[11] Y. SAAD, Iterative Methods for Sparse Linear Systems, SIAM, Philadelphia, 2003.

[12] S. MenL, Use of Picard and Newton iteration for solving nonlinear ground water flow equations, Ground Water, 44 (2006), pp. 583-594.

[13] C. T. KelLEY, Solving nonlinear equations with Newton's method, SIAM, 2003.

[14] L. A. Richards, Capillary conduction of liquids through porous mediums, J. of Physics, 1 (1931), pp. 318-333.

Edited by: Dana Petcu

Received: Nov 23, 2018

Accepted: Dec 23, 2018 


\section{AIMS AND SCOPE}

The area of scalable computing has matured and reached a point where new issues and trends require a professional forum. SCPE will provide this avenue by publishing original refereed papers that address the present as well as the future of parallel and distributed computing. The journal will focus on algorithm development, implementation and execution on real-world parallel architectures, and application of parallel and distributed computing to the solution of real-life problems. Of particular interest are:

\section{Expressiveness:}

- high level languages,

- object oriented techniques,

- compiler technology for parallel computing,

- implementation techniques and their efficiency.

System engineering:

- programming environments,

- debugging tools,

- software libraries.

\section{Performance:}

- performance measurement: metrics, evaluation, visualization,

- performance improvement: resource allocation and scheduling, I/O, network throughput.

\author{
Applications: \\ - database, \\ - control systems, \\ - embedded systems, \\ - fault tolerance, \\ - industrial and business, \\ - real-time, \\ - scientific computing, \\ - visualization.
}

\section{Future:}

- limitations of current approaches,

- engineering trends and their consequences,

- novel parallel architectures.

Taking into account the extremely rapid pace of changes in the field SCPE is committed to fast turnaround of papers and a short publication time of accepted papers.

\section{INSTRUCTIONS FOR CONTRIBUTORS}

Proposals of Special Issues should be submitted to the editor-in-chief.

The language of the journal is English. SCPE publishes three categories of papers: overview papers, research papers and short communications. Electronic submissions are preferred. Overview papers and short communications should be submitted to the editor-in-chief. Research papers should be submitted to the editor whose research interests match the subject of the paper most closely. The list of editors' research interests can be found at the journal WWW site (http://www.scpe.org). Each paper appropriate to the journal will be refereed by a minimum of two referees.

There is no a priori limit on the length of overview papers. Research papers should be limited to approximately 20 pages, while short communications should not exceed 5 pages. A 50-100 word abstract should be included.

Upon acceptance the authors will be asked to transfer copyright of the article to the publisher. The authors will be required to prepare the text in $\mathrm{IAT}_{\mathrm{E}} \mathrm{X} 2_{\varepsilon}$ using the journal document class file (based on the SIAM's siamltex.clo document class, available at the journal WWW site). Figures must be prepared in encapsulated PostScript and appropriately incorporated into the text. The bibliography should be formatted using the SIAM convention. Detailed instructions for the Authors are available on the SCPE WWW site at http://www. scpe.org.

Contributions are accepted for review on the understanding that the same work has not been published and that it is not being considered for publication elsewhere. Technical reports can be submitted. Substantially revised versions of papers published in not easily accessible conference proceedings can also be submitted. The editor-in-chief should be notified at the time of submission and the author is responsible for obtaining the necessary copyright releases for all copyrighted material. 